\title{
Thermal Compatibility and Bond Strength of FRP Reinforcement in Prestressed Concrete Applications
}

\author{
BY \\ Hugues M. Vogel \\ A Thesis \\ Submitted to the Faculty of Graduate Studies \\ In Partial Fulfillment of the Requirements for the Degree of \\ MASTER OF SCIENCE \\ Department of Civil Engineering \\ University of Manitoba \\ Winnipeg, Manitoba
}




\title{
THE UNIVERSITY OF MANITOBA
}

\section{FACULTY OF GRADUATE STUDIES \\ $\div * * * *$ \\ COPYRIGHT PERMISSION}

\section{Thermal Compatibility and Bond Strength Of FRP Reinforcement in Prestressed Concrete Applications}

\section{BY}

\author{
Hugues M. Vogel
}

\begin{abstract}
A Thesis/Practicum submitted to the Faculty of Graduate Studies of The University of Manitoba in partial fulfillment of the requirement of the degree

Master Of Science
\end{abstract}

Hugues M. Vogel (C) 2004

Permission has been granted to the Library of the University of Manitoba to lend or sell copies of this thesis/practicum, to the National Library of Canada to microfilm this thesis and to lend or sell copies of the film, and to University Microfilms Inc. to publish an abstract of this thesis/practicum.

This reproduction or copy of this thesis has been made available by authority of the copyright owner solely for the purpose of private study and research, and may only be reproduced and copied as permitted by copyright laws or with express written authorization from the copyright owner. 


\section{Table of Contents}

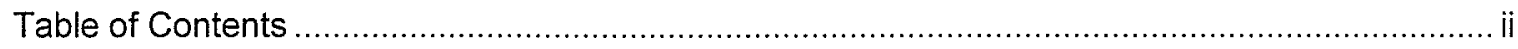

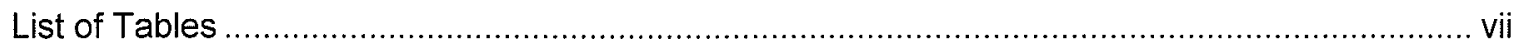

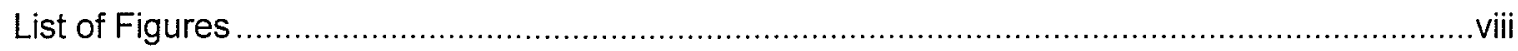

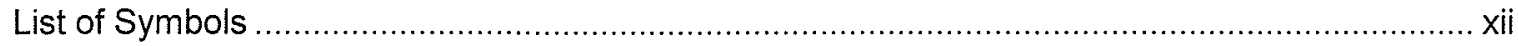

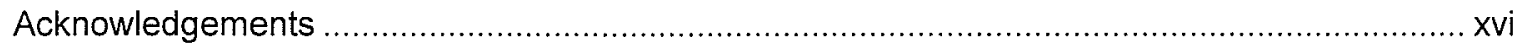

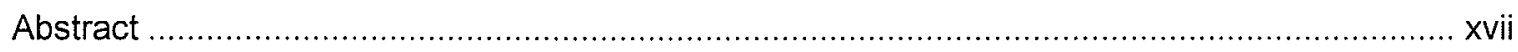

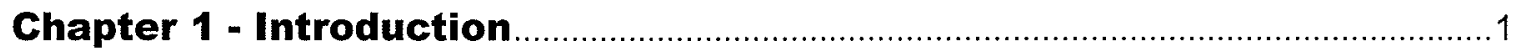

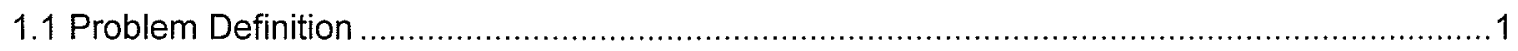

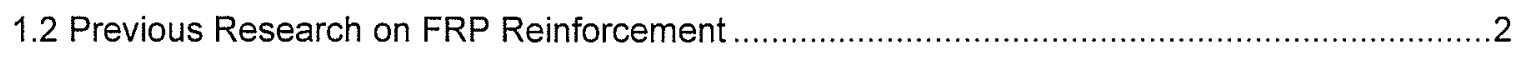

1.2.1 Effect of High Temperature on Bond Performance ............................................

1.2.1.1 Glass Transition Temperature..........................................................

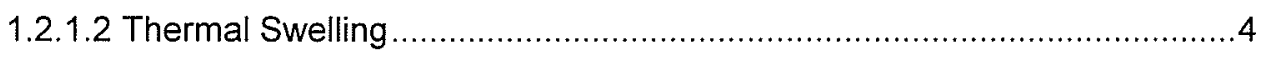

1.2.2 Linear Elastic Representation of Thermally Induced Stresses ...............................5

1.2.3 Non-linear Finite Element Representation of Thermally Induced Stresses ..............8

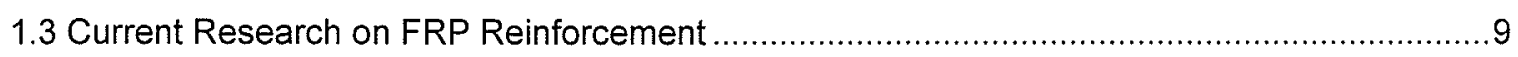

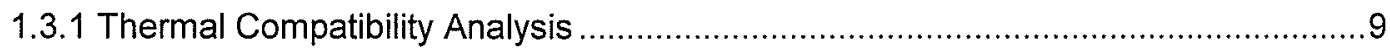

1.3.1.1 Additional Sources of Transverse Swelling .......................................11

1.3.1.2 Concrete Cover - Code Recommendations ...................................... 11

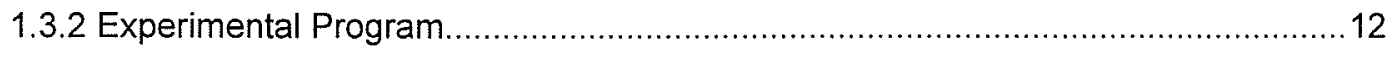

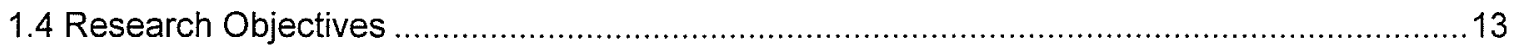

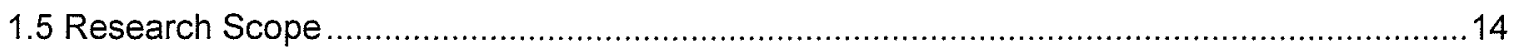




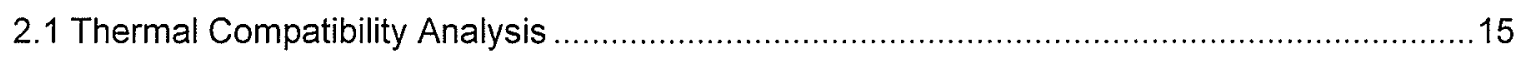

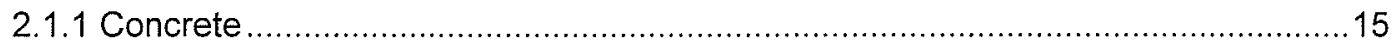

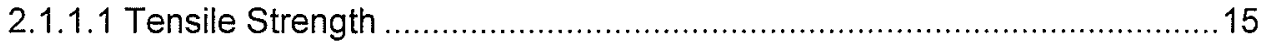

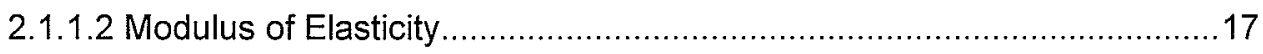

2.1.1.3 Coefficient of Thermal Expansion and Poisson's Ratio ........................18

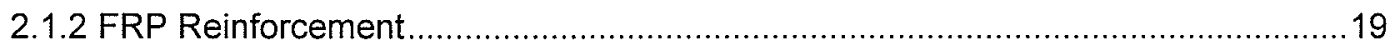

2.1.2.1 Transverse Modulus of Elasticity .................................................. 19

2.1.2.2 Transverse Coefficient of Thermal Expansion ...................................20

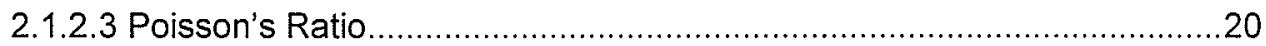

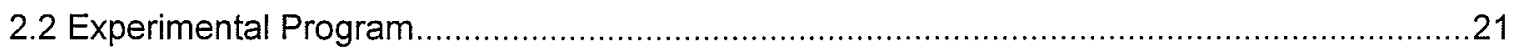

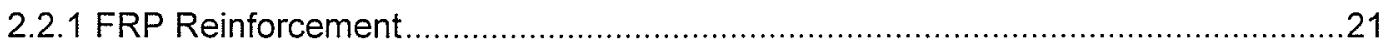

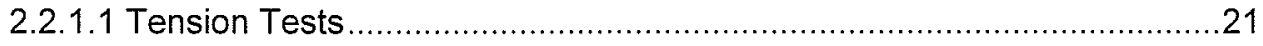

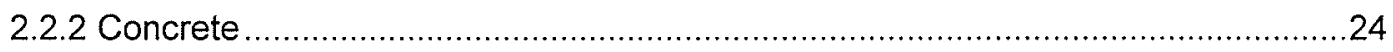

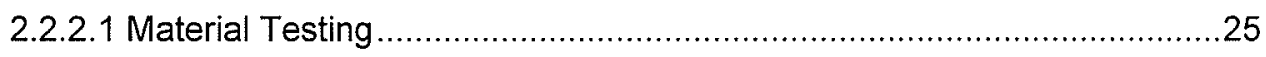

Chapter 3 - Linear Elastic Analysis of Thermally Induced Stresses ........28

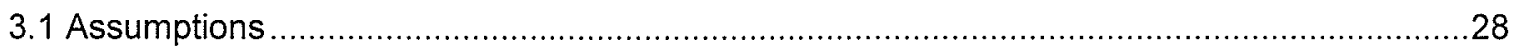

3.2 Axisymmetric Model Representation of Composite Rod Embedded in Concrete..................29

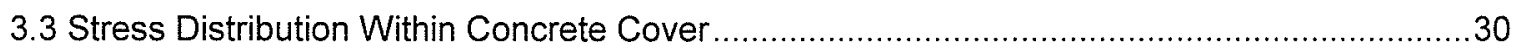

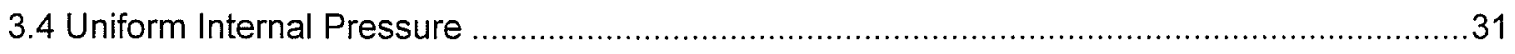

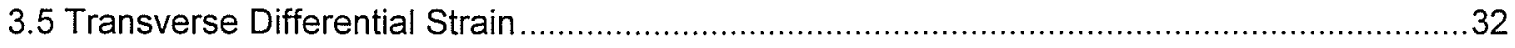

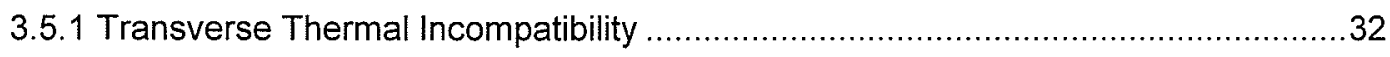

3.5.2 Longitudinal Thermal Incompatibility - Compatibility of Axial Deformation .............33

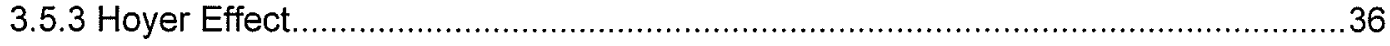

3.5.3.1 Equivalent Temperature Change …...................................................36 


\section{Chapter 3 - Linear Elastic Analysis (Continued)}

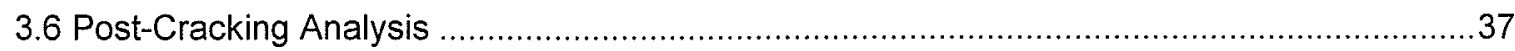

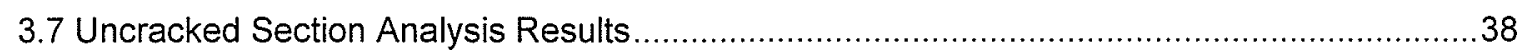

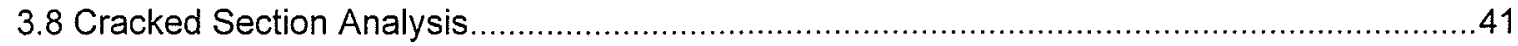

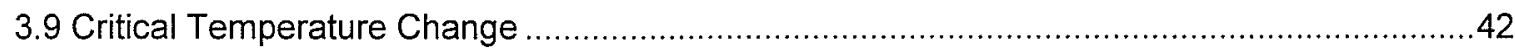

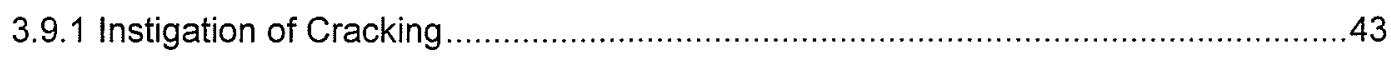

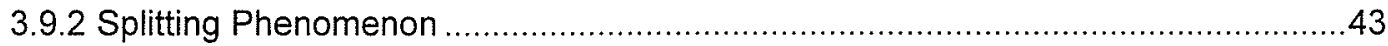

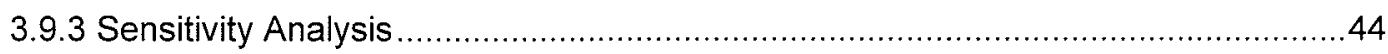

3.10 Critical Temperature Charts for Placement of FRP Reinforcement ....................................46

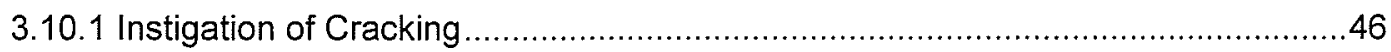

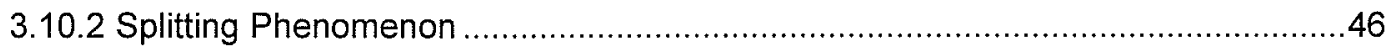

3.10.3 Procedure for Determining Minimum Concrete Cover ....................................47

Chapter 4 - Finite Element Analysis of Thermally Induced Stresses …...54

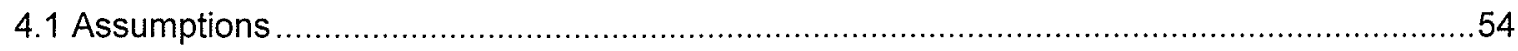

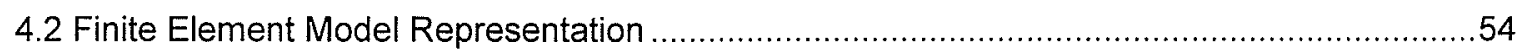

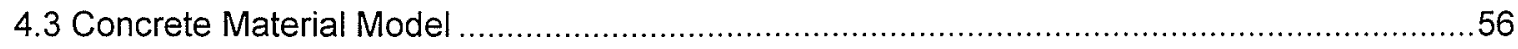

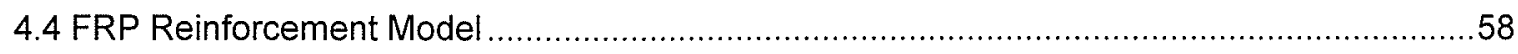

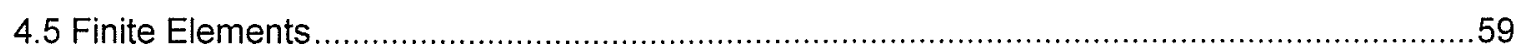

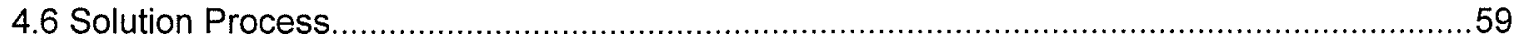

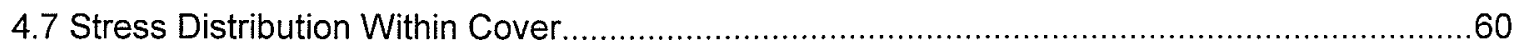

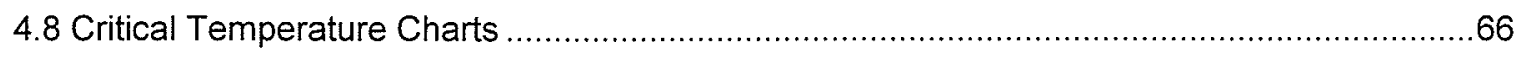

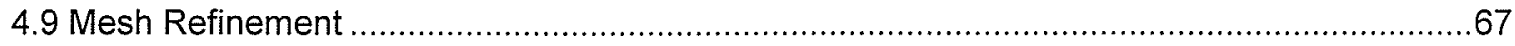




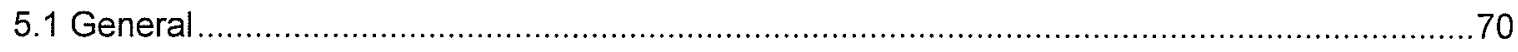

5.2 Control Beams

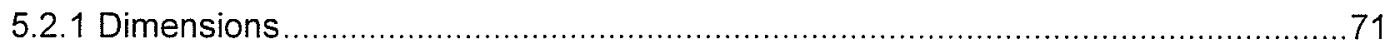

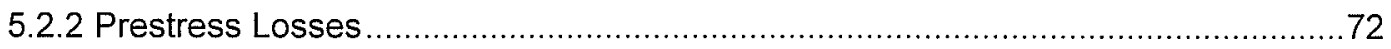

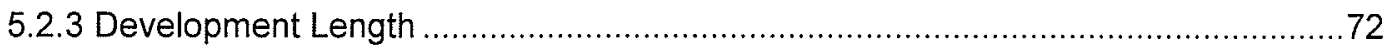

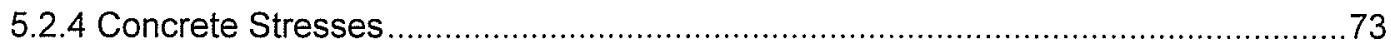

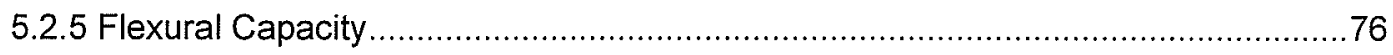

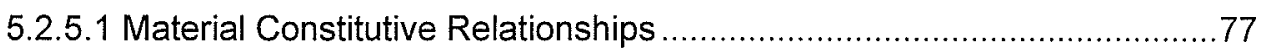

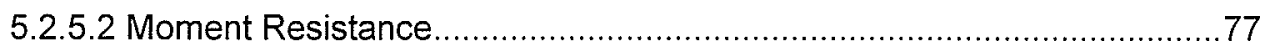

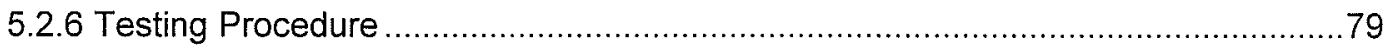

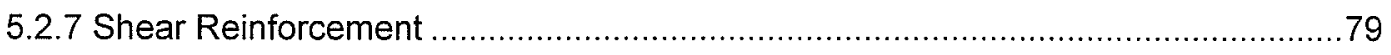

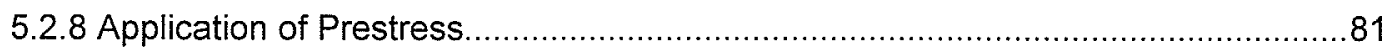

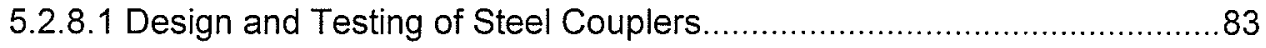

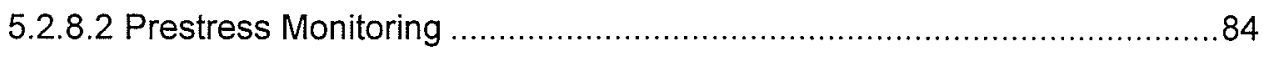

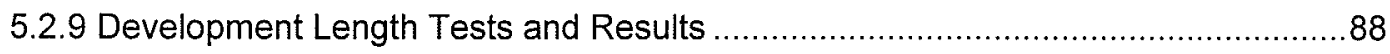

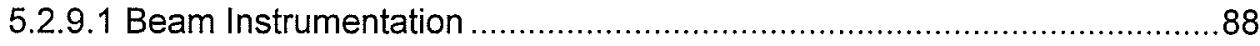

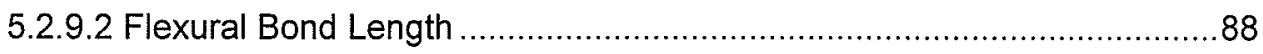

5.2.9.3 Development Length and Average Bond Stress ..............................90

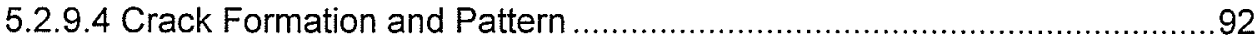

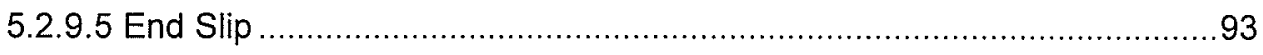

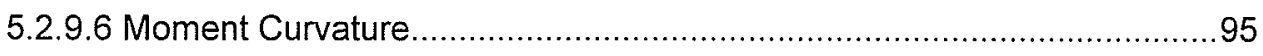

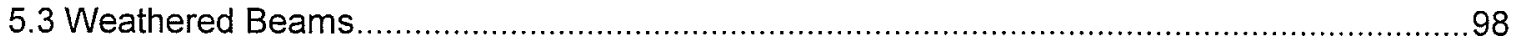

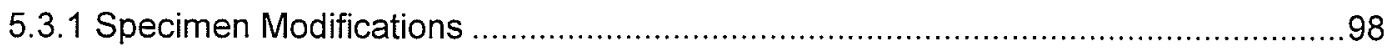

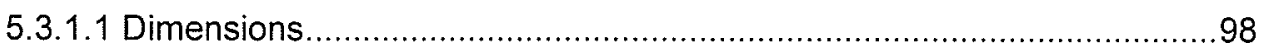

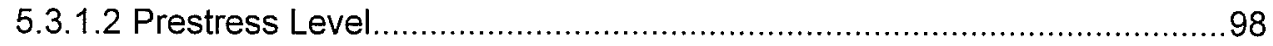

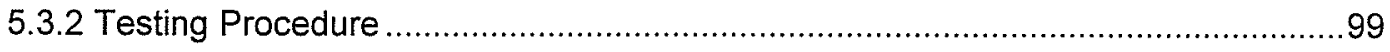




\section{Chapter 5 - Experimental Program (Continued)}

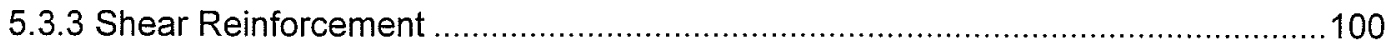

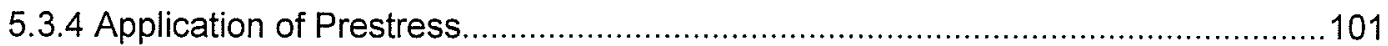

5.3.5 Hazards Involved in Prestressing Applications ............................................102

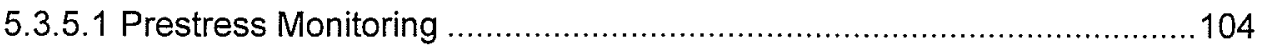

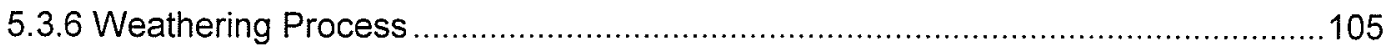

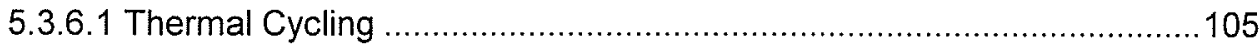

5.3.6.2 Temperature and Thermal Strain Readings ...................................106

5.3.6.3 Environmental Chamber ................................................................110

5.3.6.4 Expected Level of Damage ....................................................... 111

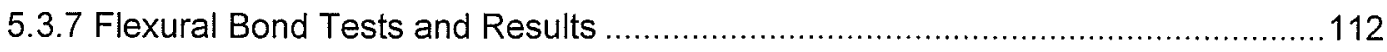

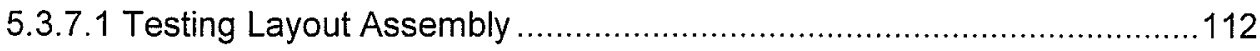

5.3.7.2 Beam Instrumentation ............................................................... 113

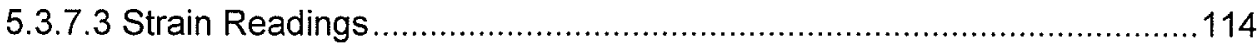

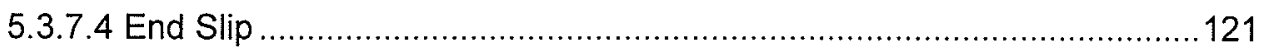

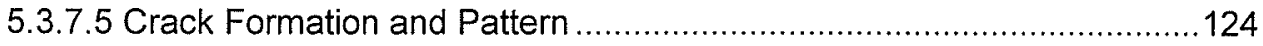

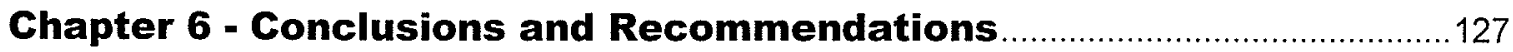

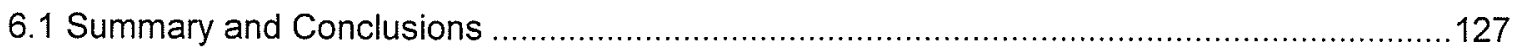

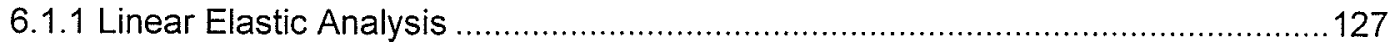

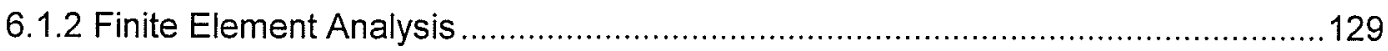

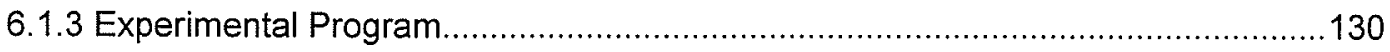

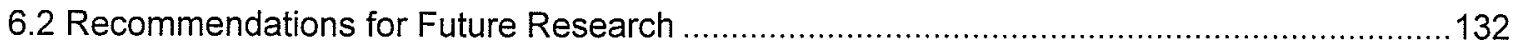

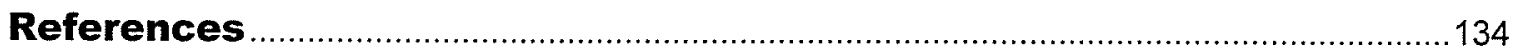




\section{List of Tables}

Table 2.1 - Reinforcement Specifications (Hughes Brothers, Inc.) …....................................21

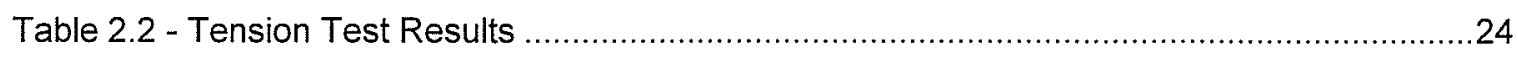

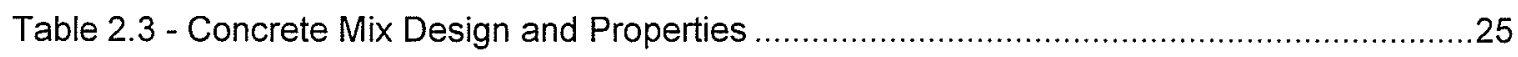

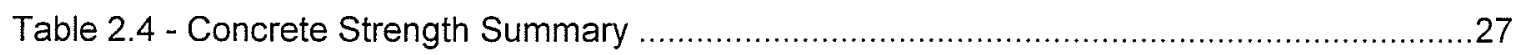

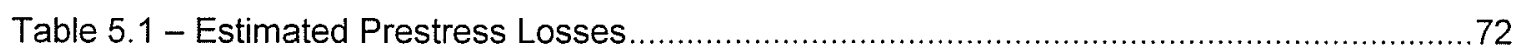

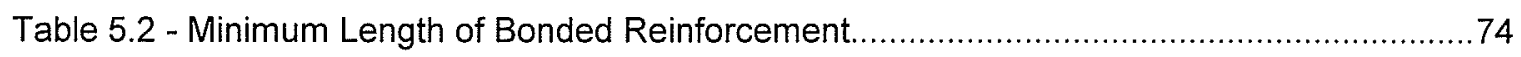

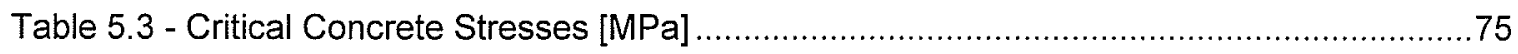

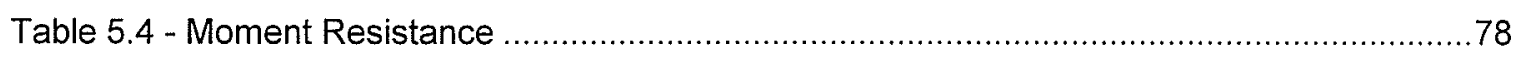

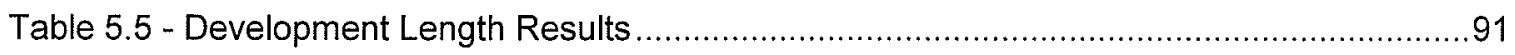

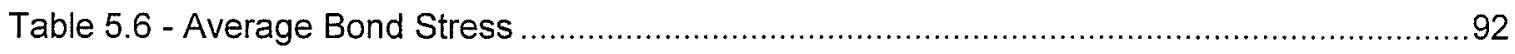

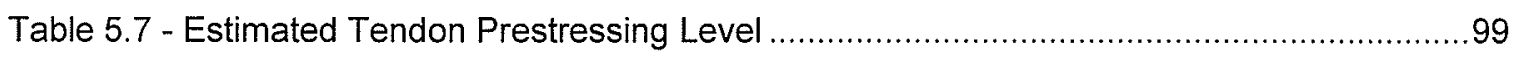

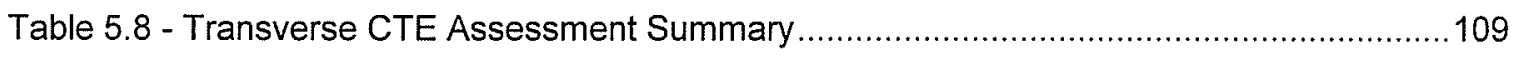




\section{List of Figures}

Figure 1.1 - FRP Reinforcement Tested for Bond Strength (Katz and Berman, 2000) ................3

Figure 1.2 - Compatibility of Axial Deformations for an FRP Reinforced Element.........................6

Figure 1.3 - Analytical Model for Crack Development and Splitting (Aiello, 1999) ......................7

Figure 1.4 - Lateral Expansion of FRP Reinforcement in Transfer Zone (Hoyer Effect) ................9

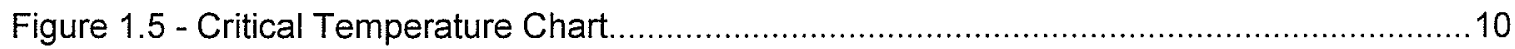

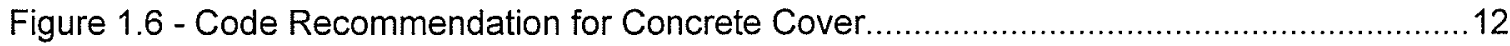

Figure 1.7 - Impact Echo Method for Locating Imperfections ..............................................13

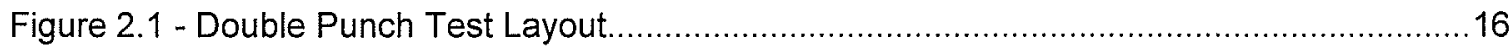

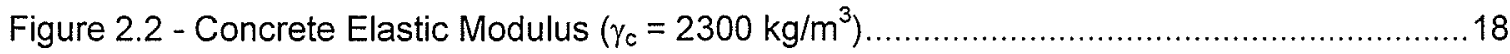

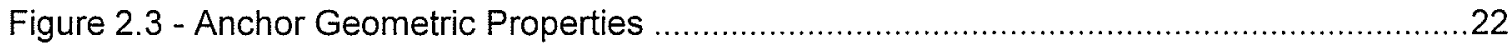

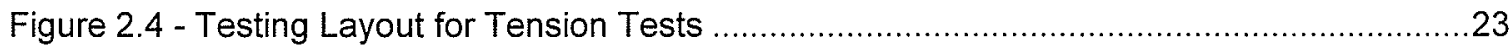

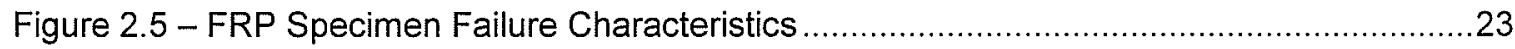

Figure 2.6 - Stress-Strain Relationship of CFRP and GFRP Reinforcement Tested...................24

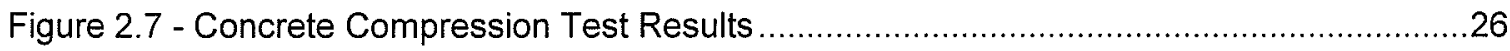

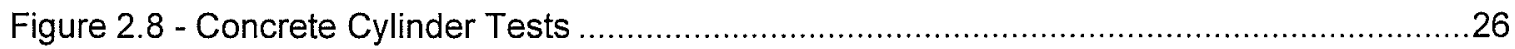

Figure 3.1 - Splitting Failure Surfaces and Model Representation ......................................29

Figure 3.2 - Composite Rod in Concrete Cylinder ............................................................

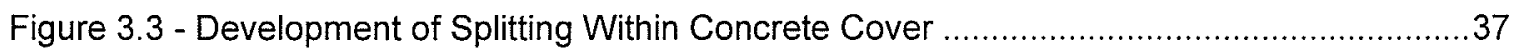

Figure 3.4 - Circumferential Stress Distribution Within Concrete Cover - Uncracked Analysis .....40

Figure 3.5 - Circumferential Stress Variation at the Interface ............................................. 41

Figure 3.6 - Circumferential Stress Distribution Within Concrete Cover - Cracked Analysis ........42

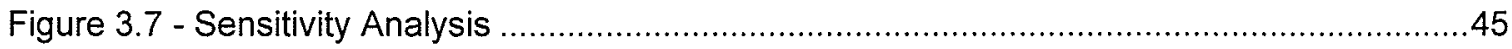

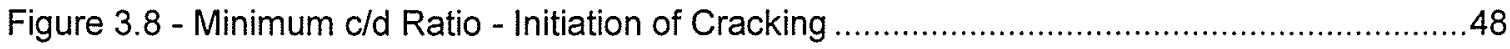

Figure 3.9 - Minimum c/d Ratio - Splitting Phenomenon......................................................51

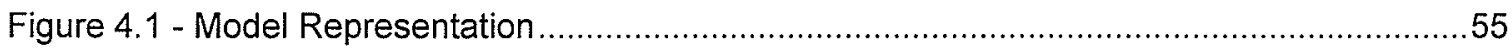




\section{List of Figures (Continued)}

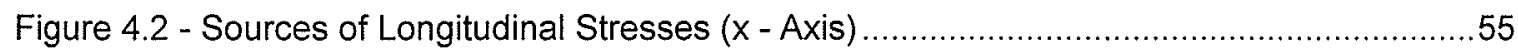

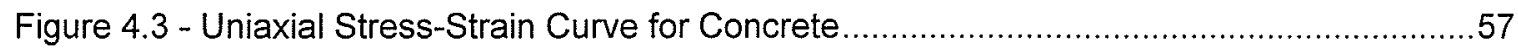

Figure 4.4 - Complete Tensile Stress-Displacement Curve ................................................57

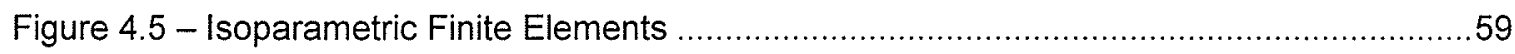

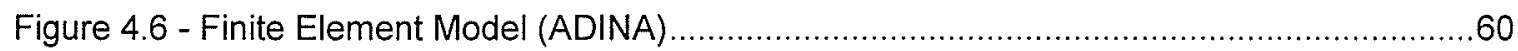

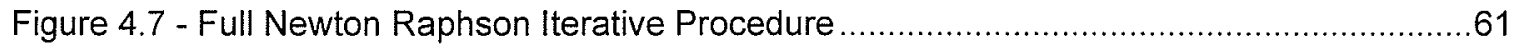

Figure 4.8 - Finite Element Analysis Programs and Boundary Conditions ................................62

Figure 4.9 - Principal Stress Distribution Within Cover (130 elements, 417 nodes) ....................63

Figure 4.10 - First Principal Stress Distribution Along Shortest Cover.....................................64

Figure 4.11 - First Principal Stress Distribution Along Diagonal Cover...................................65

Figure 4.12 - Critical Temperature Charts (Finite Element Model) .........................................67

Figure 4.13 - Finite Element Model Concrete Cover Splitting ...............................................68

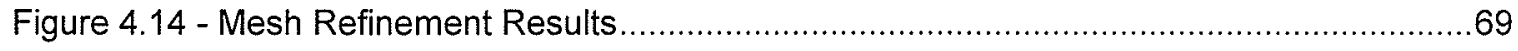

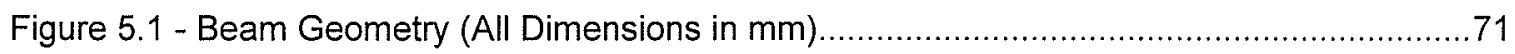

Figure 5.2 - Calculation of Concrete Stresses due to Specified Loads .......................................74

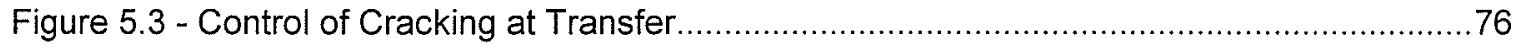

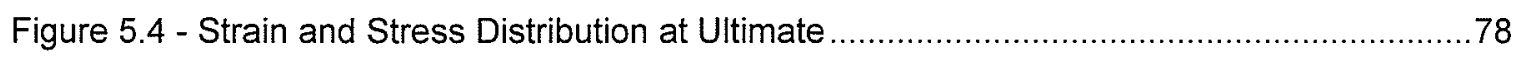

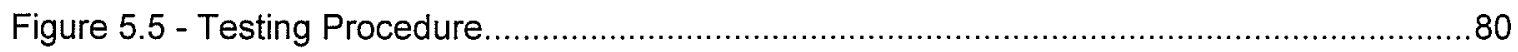

Figure 5.6 - Shear Reinforcing Scheme for Control Beams .................................................81

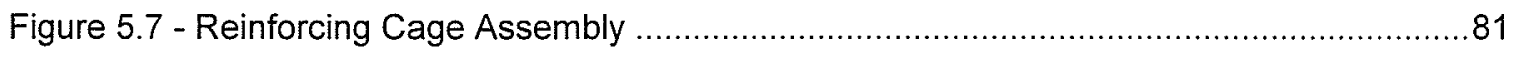

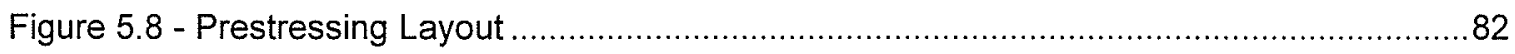

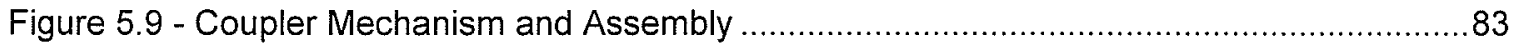

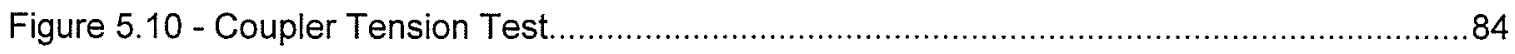

Figure 5.11 - Prestressing Loss and Transfer Length Confirmation .......................................85

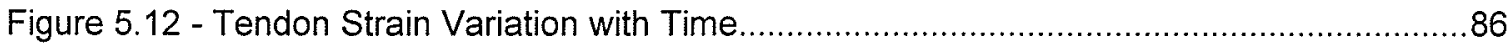




\section{List of Figures (Continued)}

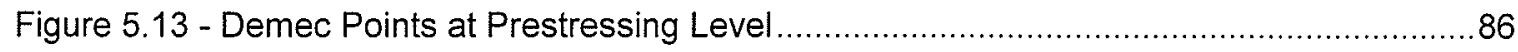

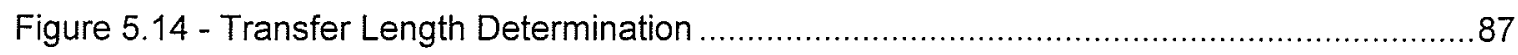

Figure 5.15 - Strain Variation at Ultimate for GFRP Prestressed Beam ....................................89

Figure 5.16 - Strain Variation at Ultimate for CFRP Prestressed Beam ................................... 89

Figure 5.17 - Bond and Tendon Stress Relationship Within Flexural Bond Length.....................90

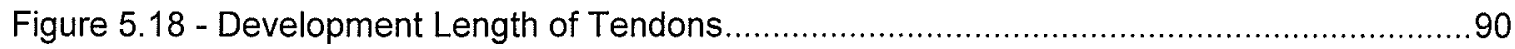

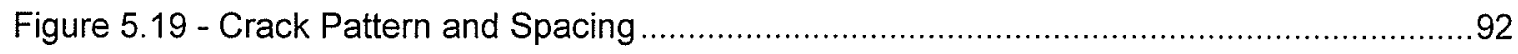

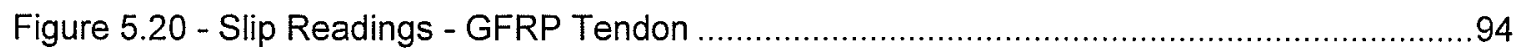

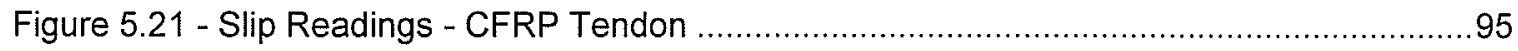

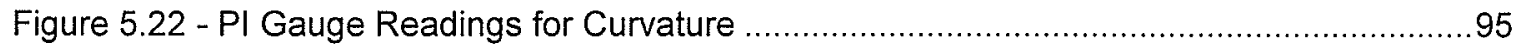

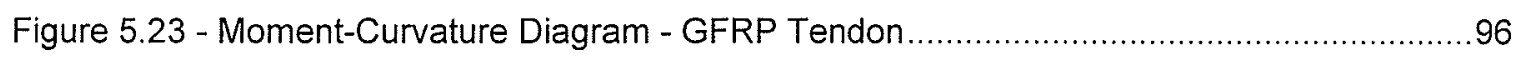

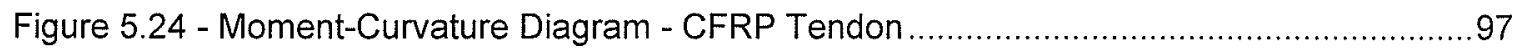

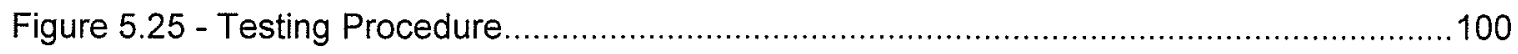

Figure 5.26 - Shear Reinforcing Scheme for Weathered Beams...........................................101

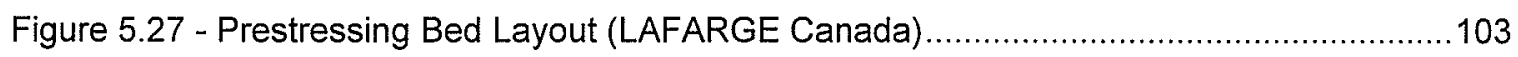

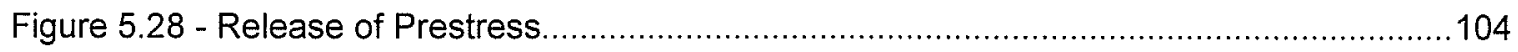

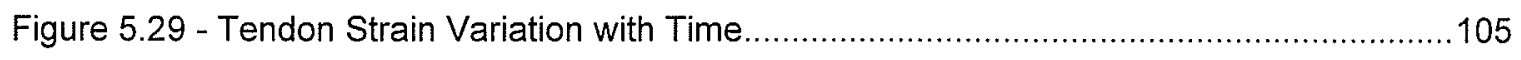

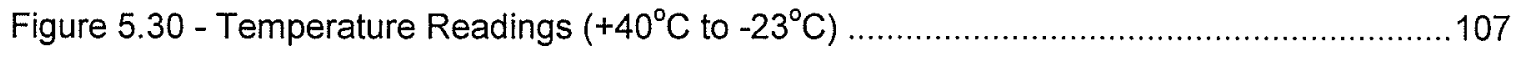

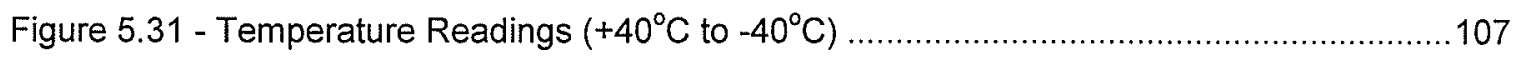

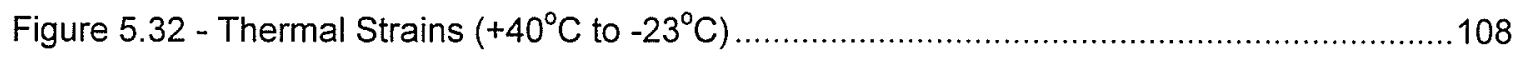

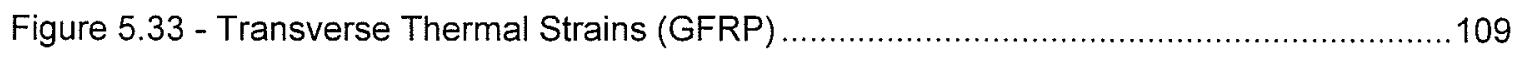

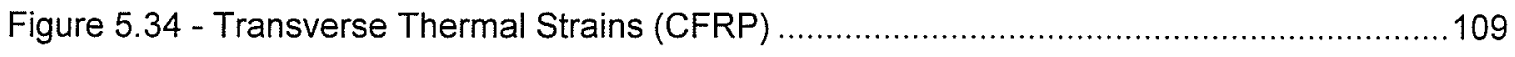

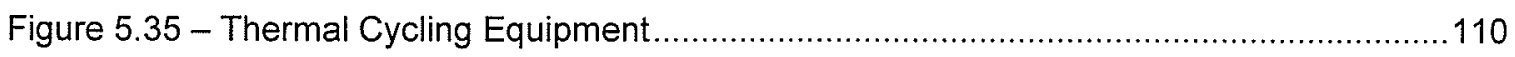

Figure 5.36 - Critical Temperature Chart $\left(f_{c}=45 \mathrm{MPa}, E_{f}^{\top}=3,000 \mathrm{MPa}, \alpha_{f}^{\top}=30 \mu \varepsilon /{ }^{\circ} \mathrm{C}\right) \ldots \ldots \ldots .111$

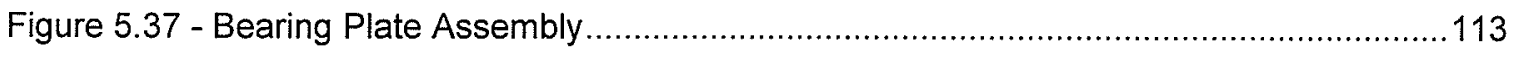




\section{List of Figures (Continued)}

Figure 5.38 - Beam Instrumentation Inside Chamber ......................................................114

Figure 5.39 - Strain Profile Along GFRP Tendon (G1-P40-S1) ….....................................115

Figure 5.40 - Environmental Chamber Testing Layout ....................................................116

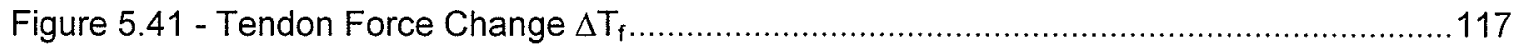

Figure 5.42 - Tendon Force Change with Applied Load ....................................................119

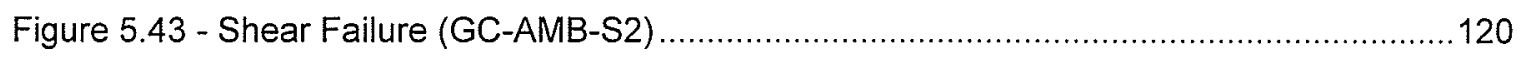

Figure 5.44 - Strain Profile Along CFRP Tendon (C1-P40-S1) ..........................................120

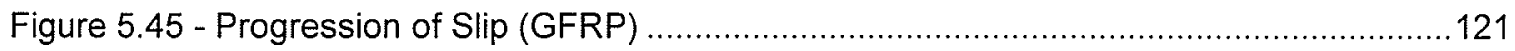

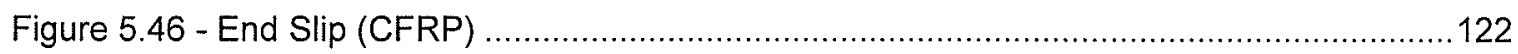

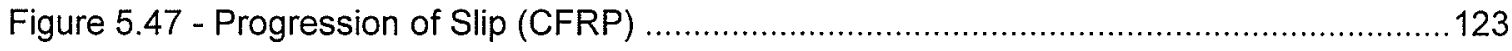

Figure 5.48 - Typical Crack Pattern and Progression - GFRP Prestressed Beams ..................124

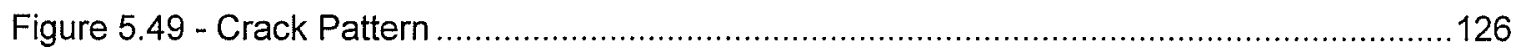




\section{List of Symbols}

a

$A_{c}$

$A_{f}$

$A_{s}$

$A_{t r}$

$\alpha \& \beta$

$\alpha_{c}$

$\alpha_{f}, \alpha_{t}$

$\alpha_{f}^{L}$

$\alpha_{f}^{\top}$

b

$b_{w}$

c

$\mathrm{CGS}_{\mathrm{tr}}$

$d_{b}$

$\delta_{\mathrm{c}}{ }^{\mathrm{L}}$

$\Delta \delta_{c}{ }^{L}$

$\delta_{\mathrm{f}}^{\mathrm{L}}$

$\Delta \delta_{f}^{L}$

$E_{c}$

$E_{f}^{\top}$

$e_{\mathrm{tr}}$

$\varepsilon^{\top}$

Bar radius $\left(d_{b} / 2\right)$

Cross sectional area of concrete cylinder [Linear Elastic Model]

Cross sectional area of FRP reinforcement [Linear Elastic Model]

Cross sectional area of compression steel

Cross sectional area of transformed section

Rectangular stress block factors

CTE of concrete

Development length regression coefficients for CFRP reinforcing bars and strands

Longitudinal CTE of FRP reinforcement

Transverse CTE of FRP reinforcement

Linear elastic model radius

Width of flexural specimens

Clear concrete cover

Section center of gravity [Transformed Section]

Bar diameter

Unrestrained longitudinal thermal deformation of concrete

Thermal deformation of concrete arising from compatibility of axial deformation

Unrestrained longitudinal thermal deformation of FRP reinforcement

Thermal deformation of FRP reinforcement arising from compatibility of axial deformation

Modulus of Elasticity of Concrete

Transverse modulus of elasticity of FRP reinforcement

Eccentricity of prestressed FRP reinforcement [Transformed Section]

Total transverse differential strain between FRP reinforcement and concrete 


\title{
List of Symbols (Continued)
}

\author{
$\varepsilon_{\mathrm{c}}^{\top} \quad$ Unrestrained transverse thermal strain of concrete \\ $\varepsilon_{\mathrm{cu}} \quad$ Ultimate compressive concrete strain \\ $\varepsilon_{\mathrm{f}}^{\top} \quad$ Unrestrained transverse thermal strain of FRP reinforcement \\ $\varepsilon_{\text {frpu }} \quad$ Ultimate tensile strain of FRP reinforcement \\ $\varepsilon_{\mathrm{po}} \quad$ Initial prestressing strain \\ $\varepsilon_{S G 2} \quad$ Strain reading from strain gauge SG2 between point of load and first support \\ [Weathered Specimens] \\ $\varepsilon_{S G 3}$ \\ Strain reading from strain gauge SG3 under the point of load [Weathered Specimens] \\ $\varepsilon_{1}^{\top}$ \\ Transverse differential strain arising from disparity of transverse CTE between FRP \\ reinforcement and concrete \\ $\varepsilon_{2}^{\top} \quad$ Transverse differential strain arising from disparity of longitudinal CTE between FRP \\ reinforcement and concrete \\ $\varepsilon_{3}{ }^{\top}$ \\ Transverse differential strain arising from Hoyer effect \\ $F_{c}$ \\ Force in concrete arising from compatibility of axial deformation \\ $f_{c}^{\prime}$ \\ Compressive Strength of Concrete \\ $f_{c s}$ \\ Stress in concrete at the level of compression steel upon release of prestress \\ $F_{f}$ \\ Force in FRP reinforcement arising from compatibility of axial deformation \\ $f_{\text {fpe }}$ \\ Effective prestressing stress in the FRP reinforcement \\ $f_{\text {fpi }}$ \\ Initial prestressing stress in the FRP reinforcement \\ $f_{\text {fpo }}$ \\ Jacking stress in the FRP reinforcement \\ $f_{\text {fpu }}$ \\ Tensile strength of the FRP reinforcement \\ $f_{t}$ \\ Tensile Strength of Concrete \\ $L_{d}$ \\ Development length of FRP reinforcement \\ $L_{\mathrm{bb}}$ \\ Flexural bond length of FRP reinforcement \\ $L_{T} \quad$ Transfer length of FRP reinforcement
}




\section{List of Symbols (Continued)}

$P_{i}$

$P_{e} \& T_{f p e}$

$p_{\text {max }}^{\prime}$

$M_{\mathrm{app}}$

$\mu_{\text {avg }}$

$\mu_{\mathrm{avg}, \mathrm{fb}}$

$\mu_{\text {avg,t }}$

$N_{c}$

$n_{s}$

$v_{\mathrm{c}}$

$v_{\mathrm{f}}^{\mathrm{ij}}$

r

$r_{\mathrm{cr}}$

$S_{\text {bot }}$

$S_{\text {top }}$

$\sigma_{r}$

$\sigma_{\theta}$

$\sigma_{\theta}^{\prime}$

$T_{\text {fpu }}$

Pressure exerted by the reinforcement on the concrete cover [Before Cracking] Arbitrary prestressing force in the FRP reinforcement

Pressure exerted by the reinforcement on the concrete cover [After Cracking] Initial prestressing force in the FRP reinforcement

Effective prestressing force in the FRP reinforcement

Maximum pressure exerted by the reinforcement on the concrete cover [Splitting]

Moment applied to the section [Including Self Weight]

Average bond stress between strain gauges SG2 and SG3 during flexural bond tests

[Weathered Specimens]

Average bond stress within flexural bond length

Average bond stress within transfer length

Tension force on the tensile face of prestressed elements upon release

Modular ratio for compression steel $\left[=E_{s} / E_{c}\right]$

Poisson's ratio of concrete

Poisson's ratio of FRP reinforcement describing lateral strain along direction j due to loading along direction $\mathbf{i}$

Radial distance away from center of reinforcement

Radius of cracked zone developing around the FRP reinforcement

Bottom section modulus [Transformed Section]

Top section modulus [Transformed Section]

Radial compressive stresses within concrete cover [Before Cracking]

Circumferential splitting tensile stresses within concrete cover [Before Cracking]

Circumferential splitting tensile stresses within concrete cover [After Cracking]

Tensile force causing rupture of the FRP reinforcement 


\section{List of Symbols (Continued)}

$\Delta T$

$\Delta T_{\text {critical,cracking }}$

$\Delta T_{\text {critical,splitting }}$

$\Delta T_{\text {equivalent }}$

$\Delta T_{f} \& \Delta T_{\text {frp }}$

$\mathrm{u}_{\mathrm{c}}$

$u_{f}$

$\Psi$
Arbitrary thermal gradient

Thermal gradient initiating cracks within the concrete cover

Thermal gradient causing splitting of the concrete cover

Equivalent thermal gradient accounting for total transverse differential strain

Tendon force change $[\mathrm{kN}]$

Length increase of concrete arising from compatibility of axial deformation

Length increase of FRP reinforcement arising from compatibility of axial deformation

Curvature obtained from flexural test data 


\section{Acknowledgements}

First, I would like to gratefully acknowledge my advisor Dr. Dagmar Svecova for her persistent support and guidance throughout the research. I am eternally grateful for her patience and appreciate the confidence and trust she set aside for me, despite all of the hardship encountered during the course of the project. She has introduced me to the many facets of structural engineering and, through her knowledge and understanding, has allowed me to grow as a person and a researcher. It has been a privilege to work under her supervision.

The Natural Sciences and Engineering Research Council is also thanked for their funding. More importantly, I thank the council for having believed in my project and supported the idea of thermal incompatibility as well as its damaging potential in civil infrastructure. Investigating, and more specifically, questioning the use of new materials is, to me, the essence of research.

For their assistance during the experimental program of the research, I profoundly acknowledge personnel working in the McQuade Structures Laboratory. I specifically thank Mr. Moray McVey, Mr. Grant Whiteside, Mr. Chad Klowak and Mr. Dino Philopulos for having willingly shared their knowledge in light of testing and preparing my specimens. I must also thank Ms. Liting Han and Ms. Evangeline Rivera for having endured, with such serenity and calmness, my lack of knowledge and the substantial stream of questions related to the operation of instruments and Data Acquisitioning Systems used during experimental testing.

I would also like to thank all of my friends and family for having always been there for me. They have been my source of inspiration from which I have learned more than I can ever imagine. Their kindness and helpfulness is forever engraved in my heart. I especially thank my parents for whom I have the utmost admiration and respect. I owe all of my accomplishments to them. They forever will be my motivation, my strength and my reason for being. I am eternally grateful to them for having helped me handle the seasons of my existence and, more importantly, for having always believed in all that I am and all that I have become. 


\section{Abstract}

Many researchers have identified the existing concern of steel corrosion in concrete structures. Noticing the rising interest of exploring fiber reinforced polymers (FRP) as reinforcement in civil infrastructure, this thesis investigates thermal incompatibility of such products with concrete. This incompatibility arises from higher potential for the material to swell under thermal gradients. Differential swelling of reinforcement and concrete causes splitting stresses to develop within the cover followed by cracking once critical temperature levels are reached. Research presented in this thesis is concerned that damage arising from cracking of the concrete cover can lead to stress relief around the reinforcement and eventual loss of bond between the materials.

Thermal stresses and associated damage within the concrete cover of structural elements reinforced with FRP were initially studied using a linear elastic analysis. The analysis revealed susceptibility of thermal stresses to the compressive strength of concrete, transverse elastic modulus and transverse coefficient of thermal expansion (transverse CTE) of the reinforcement. A finite element analysis was also performed to include nonlinear material behavior and consideration of biaxial stress states within the concrete cover. For the range of material properties considered in the project, results from both analyses were in agreement at lower concrete cover values but divergent at higher concrete cover values. More damage was predicted by the finite element analysis at higher concrete cover values. This was attributed to the consideration of compressive stresses perpendicular to splitting stresses, which can reduce the tensile strength of concrete and accelerate damage within the concrete cover.

In view of determining whether damage arising from thermal stresses was sufficient to cause reductions in bond strength of FRP reinforcement in concrete, beams with minimum concrete cover provisions were subjected to thermal gradients expected in the Canadian climate. These beams were designed according to CSA S806 (2002) specifications and requirements for the 
design and construction of concrete structures with FRP. All beams were prestressed to increase potential for damage within the concrete cover. Results from the linear elastic and finite element analyses were used to determine expected damage within the concrete cover of the beams on the basis of material properties of CFRP and GFRP reinforcement used in the experimental program as well as concrete cover provided. According to analysis results, beams were expected to crack due to the weathering process without complete splitting of the concrete cover. However, due to disputed transverse elastic modulus values used in the analyses, flexural specimens regularly monitored during the experimental program with a handheld microscope never revealed the presence of cracks within the cover. One of the GFRP prestressed beams as well as one of the CFRP prestressed beams were set aside as control specimens and were not subjected to the weathering process. Flexural bond tests conducted on all beams did not reveal a trend in bond strength between control specimens and those subjected to the weathering process. It was therefore concluded from the experimental program that CFRP and GFRP reinforcement selected for this project demonstrated excellent bond to concrete with the prestressing levels considered and minimum concrete cover values provided. 


\section{Chapter 1}

\section{Introduction}

\subsection{Problem Definition}

The greatest threat to the durability of a concrete structure is corrosion of the reinforcement. Although for many years steel bars have consistently performed well as reinforcing materials, electrochemical corrosion has become a growing concern in civil infrastructure. There has been, in response to this issue, a rising interest to explore the feasibility of using alternative corrosion resistant materials among which FRP has shown particular promise.

The composition of FRP reinforcement consists of organic or inorganic fibres embedded in a polymeric resin matrix. Many studies have observed and agreed that properties of the resulting composite material differ from that of steel reinforcement from both a mechanical and thermal point of view. These differences can compromise the compatibility of such material in a concrete environment and could be a significant obstacle to the wider use of FRP as concrete reinforcement. Since interaction between both materials is a key parameter in evaluating the structural performances of concrete structures, thermal and mechanical compatibility of FRP reinforcement must be thoroughly investigated.

From a thermal viewpoint, the little moderating effect of longitudinally orientated fibres causes excessive transverse swelling of FRP reinforcement under escalating temperatures. This thermal swelling is best described by the transverse coefficient of thermal expansion (transverse CTE), 
whose values can greatly exceed those for concrete. Conversely, the longitudinal CTE is influenced by the presence of fibres and can also reach values different from those of concrete. This additional incompatibility can cause further swelling of FRP reinforcement due to Poisson's effect.

In light of these proprietary differences, thermal compatibility of FRP reinforcement and concrete is relatively low and differential thermal swelling between the materials will lead to thermal loading followed by a state of radial and circumferential stresses at the interface. Being tensile, circumferential stresses can initiate cracking within the concrete cover and, under further temperature loading, cause propagation of cracks until complete splitting of the envelope occurs. It follows that damage within the concrete cover arising from thermal cracking can potentially affect bond interaction between the reinforcement and concrete. Previous work done in the field of thermal compatibility and bond performance of FRP reinforcement in concrete should therefore be reviewed and discussed to line up the current project in determining the effects of thermal gradients on bond strength.

\subsection{Previous Research on FRP Reinforcement}

\subsubsection{Effect of High Temperature on Bond Performance}

Many researchers have investigated bond performance of FRP reinforcement in comparison to that of conventional steel. Katz (1999) investigated the bond strength of four types of glass FRP (GFRP) reinforcing bars each having a different surface treatment and bond mechanism to concrete. As illustrated in Figure 1.1, one of the reinforcing bars had moulded deformations as those commonly found on the surface of steel reinforcement while a braid of fibres helically wrapped the others. Two of the wrapped bars were sand coated while the third achieved roughness after polymerisation of excess polymer on the surface. The bond strength was determined on the basis of a pullout test conducted in accordance with ASTM D3418 and D5028. 
At room temperature, the results demonstrated that FRP reinforcement had comparable bond strength in concrete than that of conventional steel.

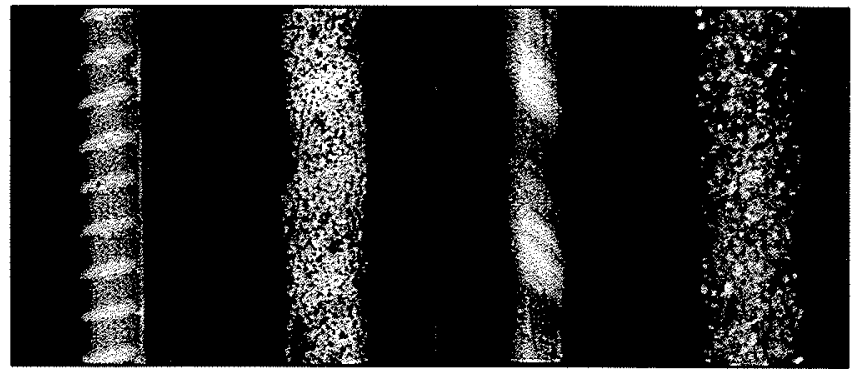

(a) Surface Treatment

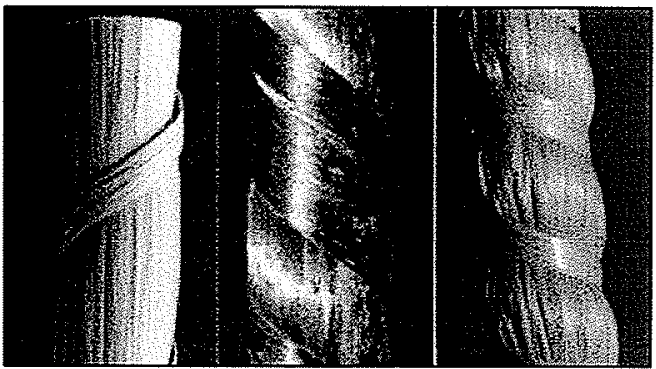

(b) Fibre Braid Wraps

Figure 1.1 - FRP Reinforcement Tested for Bond Strength (Katz and Berman, 2000)

\subsubsection{Glass Transition Temperature}

Once the glass transition temperature is reached, the polymeric matrix holding the fibres will begin to lose its mechanical properties. Further investigation by Katz and Berman (2000) on FRP reinforcing bars with polyester and vinylester resin matrices revealed a strong reduction in bond strength when the temperature was increased beyond $80^{\circ} \mathrm{C}$. However, the bond strength was observed to stabilize at a constant residual value for temperatures above $200^{\circ} \mathrm{C}$. Moreover, the bond strength of FRP reinforcement was found to reduce to approximately $10 \%$ of its initial value while a reduction of only $30 \%$ was seen for conventional steel at temperatures close to $200^{\circ} \mathrm{C}$. It also became apparent from the results of the research that reinforcing bars wrapped with a braid of fibres were less sensitive to high temperatures than those containing moulded deformations. This was attributed to the use of helical wraps that maintain their contribution to bond strength as the matrix gradually loses its properties at elevated temperatures.

Although temperatures approaching the glass transition temperature can be reached during a fire, the current research is concerned with temperatures occurring within the Canadian climate. These temperatures rarely exceed $40^{\circ} \mathrm{C}$ and remain distant from that expected in a fire. Keeping 
in mind the potentially damaging effect of fire for future research, the current project will therefore consider loss in bond strength of FRP reinforcement due to thermal swelling at temperature levels lower than the glass transition point.

\subsubsection{Thermal Swelling}

Due to the alignment of fibres in the axial direction only, mechanical and thermal properties of FRP reinforcing materials will vary with orientation. More specifically, properties in the longitudinal direction will be significantly influenced by the presence of fibres while the resin will have a considerable effect on those in the transverse direction. Due to the little moderating effect of fibres in the transverse direction, the transverse CTE of FRP reinforcement will be mainly affected by considerable swelling of the resin matrix under thermal action. As emphasized by Aiello (1999), the CTE values of several FRP reinforcing bars can reach up to eight times that of concrete causing transverse swelling of both materials to be relatively different when considering temperature gradients. As a consequence of thermal loading, this incompatibility will generate a state of radial and circumferential stress within the concrete cover.

Circumferential stresses being tensile, thermal expansion of FRP reinforcing bars may cause initiation and development of cracking within the concrete cover of structural elements. As the temperature increases, these longitudinal cracks will propagate away from the reinforcement towards the external surface of structural elements until complete splitting of the cover occurs. The splitting phenomenon has been widely analysed for traditional steel reinforced concrete members where stresses are transferred between concrete and surface deformations of the reinforcement during loading. Studies concerned with this mechanism of stress transfer have suggested that development of longitudinal cracks within the cover of structural elements can cause an abrupt drop in bond strength followed by a brittle bond failure (Avalle et al. 1993). 


\subsubsection{Linear Elastic Representation of Thermally Induced Stresses}

Rahman et al. (1995) investigated thermal stresses that occur in FRP reinforced concrete elements and the main parameters affecting their development. The research clearly identified the anisotropic nature of FRP reinforcement and found that the modulus of elasticity and CTE of the material in the transverse orientation were the key properties affecting thermal stresses within the cover. Establishing these properties on the basis of experimental work and confirming with theoretical estimates, the research also considered thermal incompatibility in the longitudinal direction and its contribution to thermally induced stresses.

Noticing that fibres will influence thermal swelling of FRP reinforcing bars in the longitudinal orientation and cause CTE values to differ from that of concrete in this direction, Rahman et al. (1995) considered compatibility of axial deformations between the materials. As indicated by the study and illustrated in Figure 1.2, inclusion of this requirement was found to cause an increase in transverse swelling of the reinforcing bar with larger thermal stresses within the cover for the case of longitudinal CTE values higher than that of concrete. Conversely, longitudinal CTE values lower than that of concrete revealed a decrease in transverse swelling with smaller thermal stresses developing within the cover. This additional value for transverse swelling was incorporated in a linear elastic axisymmetric formulation initially developed by Timoshenko and Goodier (1970) to investigate thermal stresses within the cover of a concrete cylinder surrounding the reinforcement. The analysis concluded that thermal stresses arising from typical temperature gradients in the Canadian climate could instigate cracking in concrete elements reinforced with FRP, possibly leading to reductions in bond strength.

Although the model proposed by Rahman et al. (1995) was capable of predicting crack initiation within the concrete cover of structural elements, it was unable to predict the extent of cracking and related stress relief around the reinforcement. Consequently, the model was later modified by Nanni et al. (2001) to incorporate a post-cracking analysis. The modification allowed the 
analysis to consider development of cracking within the concrete cover and determine critical temperature changes causing the splitting phenomenon. The analysis was verified experimentally by placing glass FRP (GFRP) reinforced specimens in an oven and subjecting them to thermal loading. Temperature was increased in increments of 5 to $10^{\circ} \mathrm{C}$ until cracks appeared on the external surface of the specimens.

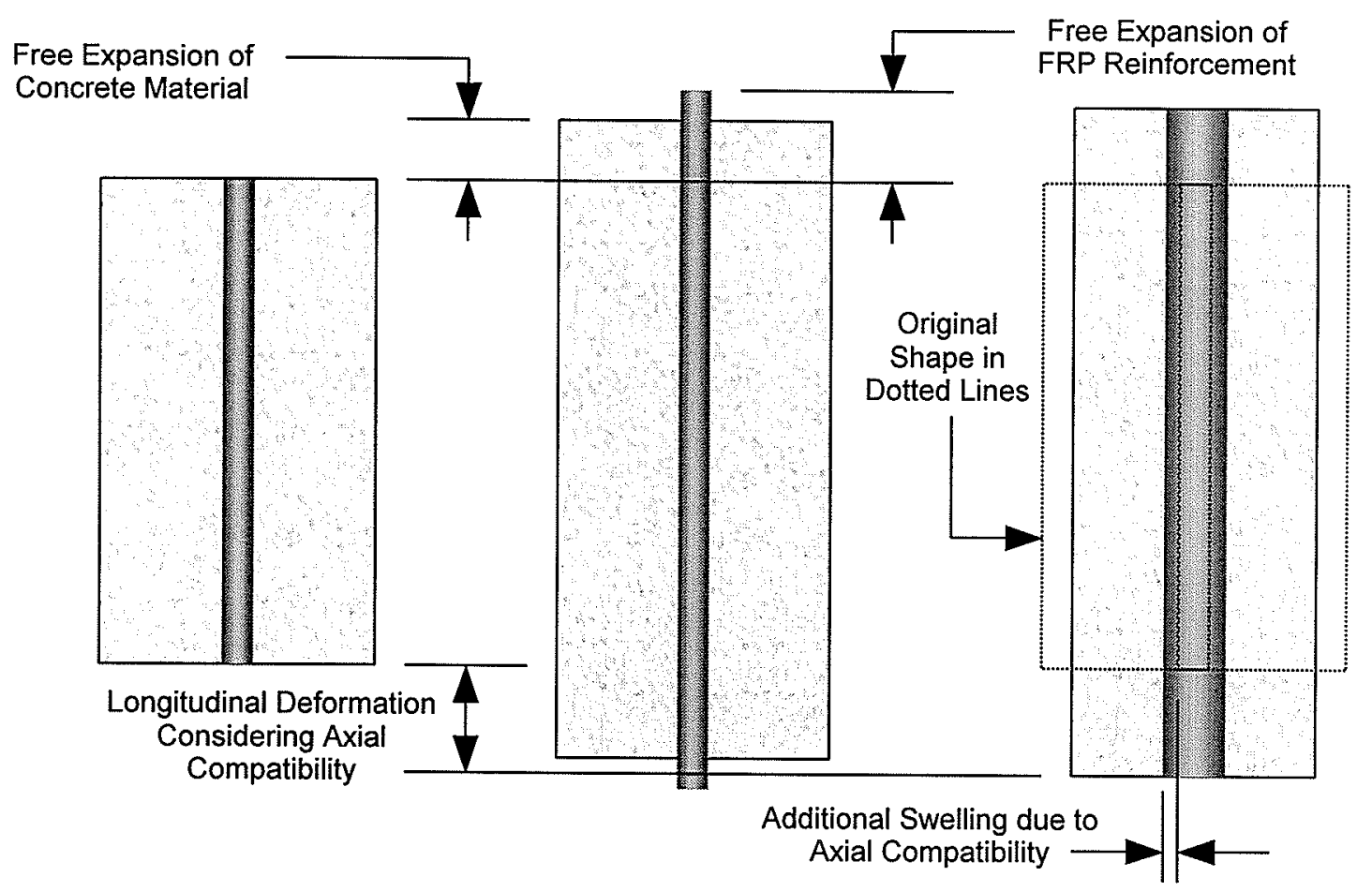
(a) Original Shape
(b) Free Expansion
(c) Requirement of Compatibility

Figure 1.2 - Compatibility of Axial Deformations for an FRP Reinforced Element

Specimens with rectangular and circular cross-sections were considered for the experimental program. The research concluded that critical temperature gradients producing spalling of the concrete cover were in good agreement with the analytical predictions. However, the research emphasized that a wider experimental investigation was required to assess the accuracy of the analytical model and effect of cross-sectional shape on the development of cracking. 
Aiello (1999) further investigated the development of thermal stresses within the cover of concrete elements reinforced with FRP by conducting a similar analysis. The analysis was carried out by defining possible failure mechanisms of the concrete cover, removing axisymmetry from the thermal stress problem previously assumed by Rahman et al. (1995). Assuming linear elastic material behaviour, the analytical procedure consisted of dividing the concrete cover into triangular elements and determining the thermal loading at splitting on the basis of limiting conditions. The model presented by Aiello (1999) is shown in Figure 1.3.

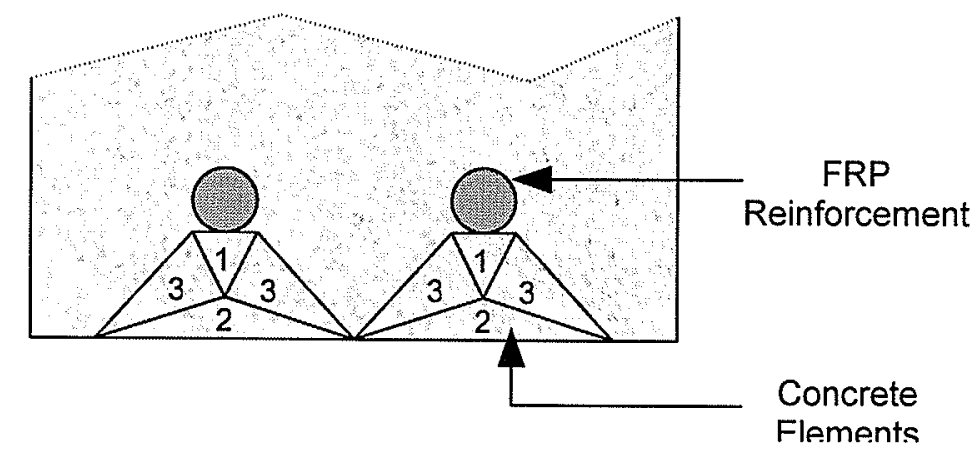

Figure 1.3 - Analytical Model for Crack Development and Splitting (Aiello, 1999)

An experimental program consisting of $100 \mathrm{~mm}$ wide by $150 \mathrm{~mm}$ deep concrete members reinforced with aramid FRP (AFRP) reinforcement was undertaken to verify the proposed model at various concrete cover values. The specimens were subjected to thermal gradients inside a furnace until longitudinal cracks appeared on the external surface. For the two levels of concrete compressive strengths considered in the research, it became apparent that the model was in agreement with experimental results for small concrete cover values describing concrete cover to bar diameter (c/d) ratios below 2.0. However, for larger concrete cover values defining $\mathrm{c} / \mathrm{d}$ ratios beyond 2.0 , the critical temperature experimentally observed was consistently lower than that predicted by the analytical model.

Aiello (1999) attributed the divergence between experimental and theoretical results to the presence of nonlinear phenomena, which was not considered in the model and may lead to a different redistribution of stresses between concrete and reinforcement. The concluding remarks 
of the research stressed the need for improvement of the theoretical model by incorporating nonlinear phenomena and evaluating its effect on concrete cover failure of FRP reinforced concrete structures subjected to thermal loading.

\subsubsection{Non-linear Finite Element Representation of Thermally Induced Stresses}

In light of conclusions obtained from the use of linear elastic thermal stress expressions, de Schutter et al. (1997) examined a two-dimensional non-linear finite element analysis of thermal incompatibility between FRP reinforcement and concrete. The research was specifically concerned with the high transverse CTE of AFRP reinforcement and thermal stresses that subsequently develop within the cover. Prestressed prisms with equal sides were considered in the analysis since release of prestress is known to cause additional transverse swelling of the reinforcement in a region called the transfer zone at the ends of structural elements. The wedge shaped expansion of the prestressing element in this zone is illustrated in Figure 1.4 and better known as the Hoyer effect. It was introduced by de Schutter et al. (1997) as an equivalent thermal loading.

Taking into account symmetry of the geometry, thermal swelling of reinforcement and boundary conditions, a quarter of the cross-section was modelled by means of two-dimensional plane stress elements. Triangular elements consisting of 6 nodes were used to partition the AFRP reinforcement while rectangular elements delimited by 8 nodes were used to break up the concrete cover. Tension softening of the concrete material was modelled by specifying the fracture energy $G_{f}$, described as the area under the softening branch. Recalling the presence of aggregates in concrete and corresponding shear stresses that can still develop inside a crack, a variable shear retention factor was used in the analysis.

It was clear from the finite element analysis that transverse thermal expansion of FRP reinforcement had a considerable influence on the concrete cover required to prevent occurrence 
of the splitting phenomenon. The researchers recognized the importance of considering the Hoyer effect by noticing a $50 \%$ increase in the concrete cover required to prevent the splitting phenomenon under a simultaneous temperature increase of $60^{\circ} \mathrm{C}$. However, the research recognized that the concrete compressive strength had a considerable effect on and strong potential in preventing the development of cracks and splitting of the cover. The Hoyer effect on critical concrete cover was found to reduce by $27 \%$ when increasing the compressive strength from 45 to $90 \mathrm{MPa}$.

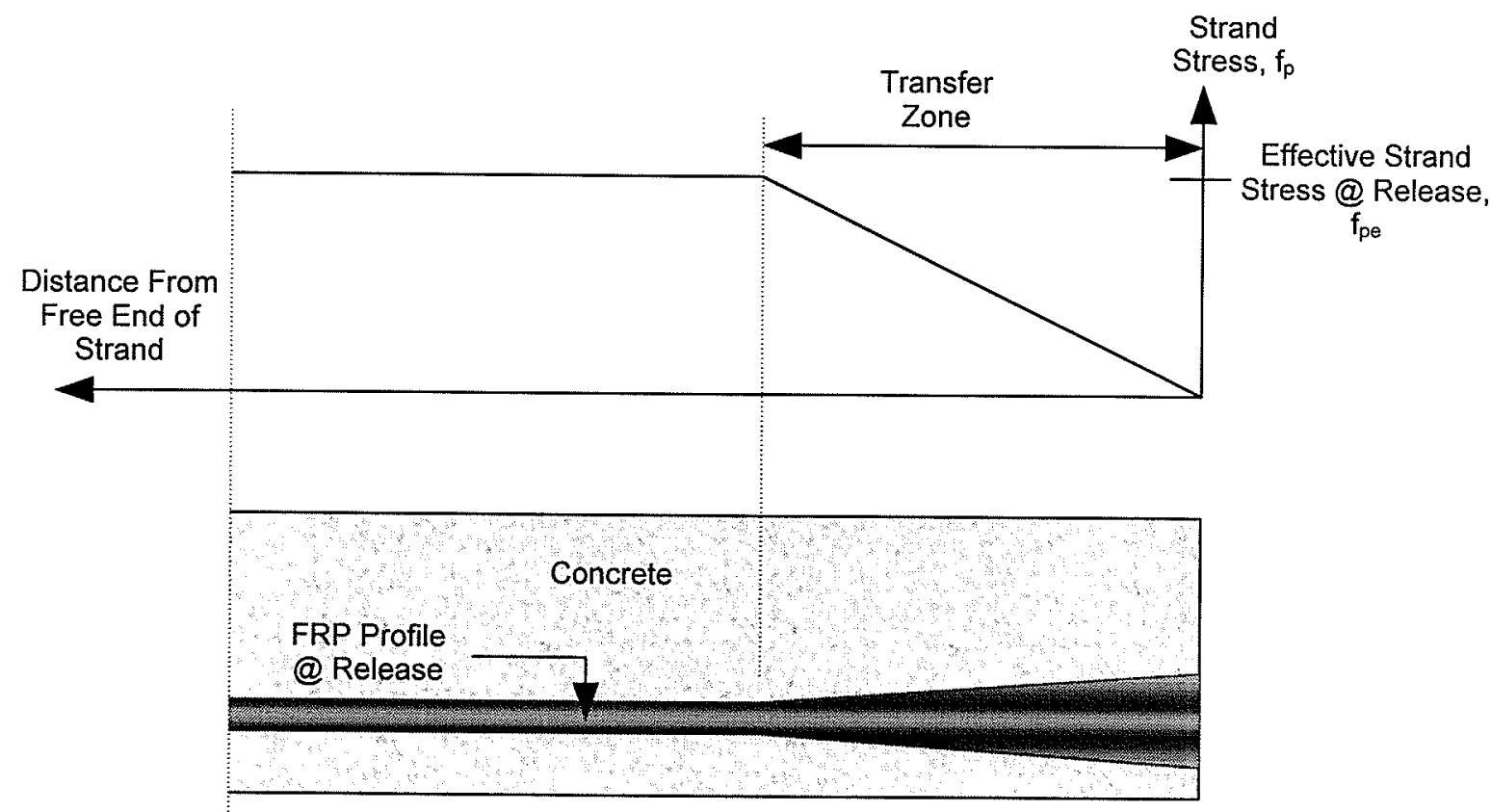

Figure 1.4 - Lateral Expansion of FRP Reinforcement in Transfer Zone (Hoyer Effect)

\subsection{Current Research on FRP Reinforcement}

\subsubsection{Thermal Compatibility Analysis}

From the previous discussion, it is apparent that splitting of the concrete cover arising from thermal swelling of FRP reinforcement has already been widely analysed. However, parameters affecting the analyses are widely scattered among the many research articles reviewed, making 
the interpretation of conclusions a difficult task. These parameters include material behaviour as well as the choice of material and geometrical properties. It was therefore decided for the current project to gather and consider these parameters in linear elastic and finite element analyses describing thermal swelling of FRP reinforcement. In addition, comparison of results from both analyses and broadening of the study to account for a wide range of material properties will bring completeness to the topic of thermal compatibility. This can also clarify some of the issues encountered in past research. These issues mainly relate to the lack of non-linear material behaviour and the limitation on the variety of section geometries that can be considered in analytical models based on linear elastic expressions only.

In this thesis, compatibility of FRP reinforcement in a concrete environment is expressed as the potential of the reinforcing material to initiate and develop cracks within its surrounding when subjected to thermal gradients. Analyses presented herein were therefore used to determine thermal gradients causing crack initiation in and complete splitting of the concrete cover. The process allowed for establishing the temperature range where cracks can propagate within the reinforced element and cause bond interaction between the materials to deteriorate. Critical temperature charts as shown in Figure 1.5 were developed to define this range for a given set of concrete cover values and elaborated to consider mechanical and thermal properties for a wide range of concrete and commercially available FRP products.

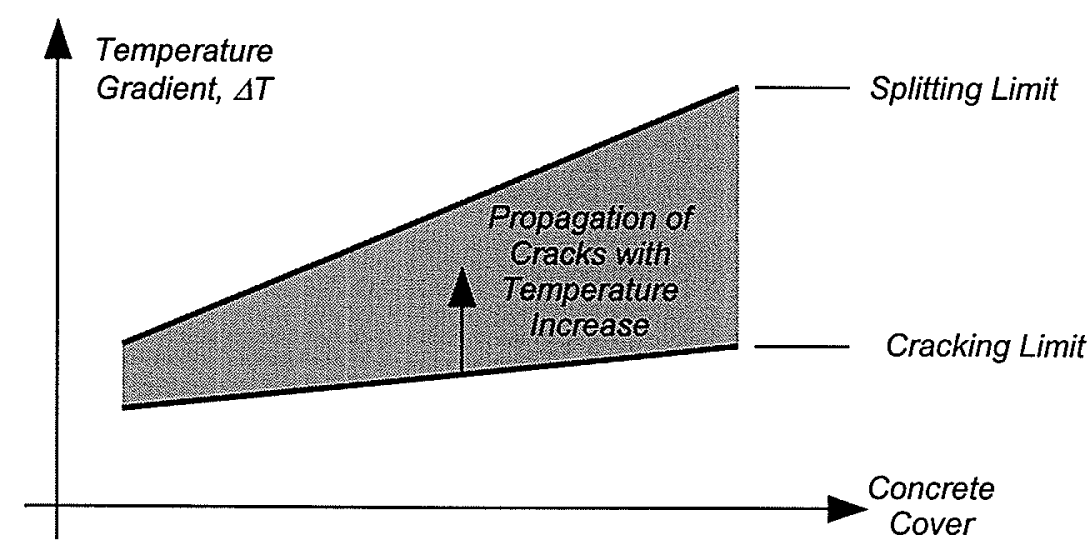

Figure 1.5 - Critical Temperature Chart 


\subsubsection{Additional Sources of Transverse Swelling}

It has been previously established that disparity of longitudinal CTES between FRP reinforcement and concrete as well as the Hoyer effect in the transfer region of prestressed elements will cause additional transverse swelling of the reinforcement. Accordingly, the research will be broadened to incorporate these additional sources of thermal incompatibility into the analyses. Methods accounting for longitudinal CTE disparity are based on expressions provided by Rahman et al. (1995) while those accounting for the Hoyer effect were initially studied by de Schutter et al. (1997).

\subsubsection{Concrete Cover - Code Recommendations}

In prescribing the minimum clear cover for pretensioned concrete members, CSA S806-02 takes into account the effect of temperature expansion and the Hoyer effect in the transfer region. According to the code, the minimum clear cover should be taken as the product of 3.5 and the bar diameter or $40 \mathrm{~mm}$, whichever is greatest. For bar diameters below $11.5 \mathrm{~mm}$, the former clear cover requirement will govern while for bar diameters greater than $11.5 \mathrm{~mm}$, the latter will govern. As a result, under no circumstances can the concrete clear cover to bar diameter ratio (c/d) fall below 3.5. It can, however, reach values up to 6.5 for the smaller bar diameters commercially available. Figure 1.6 illustrates this aspect of the requirement.

Noticing the growing interest for reducing concrete covering due to non-susceptibility of FRP reinforcement to corrosion, the analyses presented in this thesis will also investigate the use of $\mathrm{c} / \mathrm{d}$ ratios below 3.5. The $\mathrm{c} / \mathrm{d}$ ratios considered in this thesis were therefore chosen to range between 1.0 and 6.5 . 


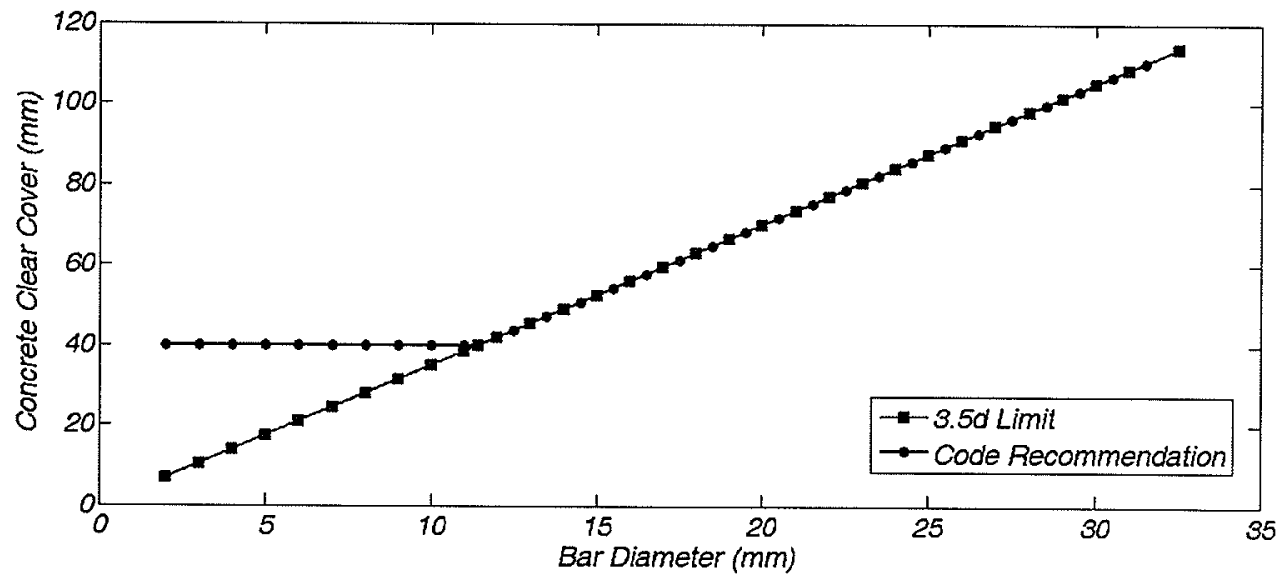

(a) Concrete Clear Cover

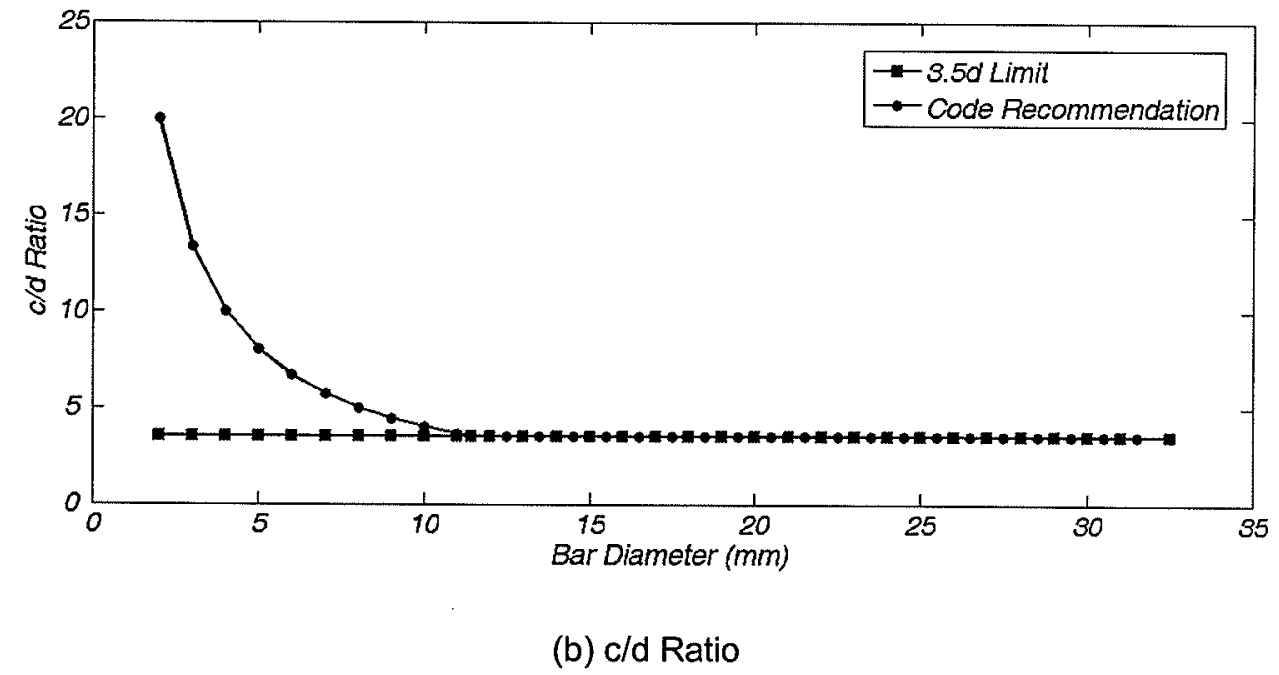

Figure 1.6 - Code Recommendation for Concrete Cover

\subsubsection{Experimental Program}

The experimental program of this thesis will consist of subjecting beams prestressed with FRP to thermal gradients expected in the Canadian climate. Beams will be prestressed to incorporate additional sources of transverse swelling and stresses within the concrete cover due to the Hoyer effect. Flexural design was based on provisions from the CSA S806-02 code for the design and 
construction of building components with FRP. Requirements pertaining to the prestressing level and concrete cover were taken into account in the design process.

It is expected for the development of cracks within the concrete cover to cause stress relief around the reinforcement followed by deterioration of the bond between the materials. Beams will therefore be tested in flexure to evaluate bond strength remaining after thermal action. Conceivable reduction in bond strength will be related to the degree of compatibility between the reinforcing material and concrete as obtained from thermal analyses performed in this thesis.

Finally, the extent of cracking within the concrete cover of experimental specimens will be assessed using a wave propagation method called Impact Echo, illustrated in Figure 1.7. To monitor the progression of damage within the concrete envelope during the course of the experimental program, specimens will be subjected to the method after release of prestress and after thermal loading.
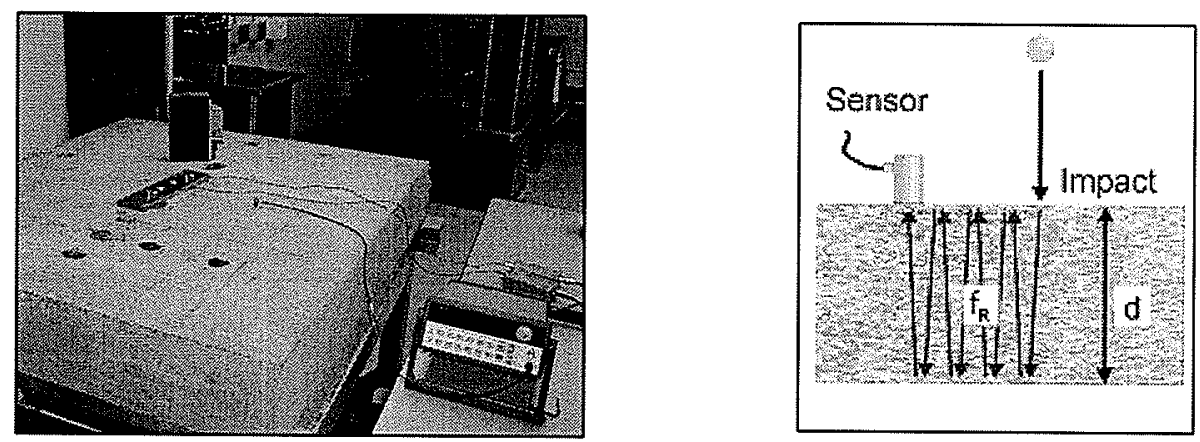

Figure 1.7 - Impact Echo Method for Locating Imperfections

\subsection{Research Objectives}

Objectives of the research were established on the basis of arguments presented in previous sections and are stated in more detail below. They include goals set out for the thermal analyses as well as for the experimental program of the current project. 
(1) Establish thermal incompatibility of FRP reinforcement in a concrete environment and associated damage through cracking on the basis of a thorough analysis considering thermal loading and stresses arising from differential swelling of both materials.

(2) Investigate the effect of thermal incompatibility and related damage on the interaction of FRP reinforcement and concrete by experimentally measuring the bond strength remaining in flexural elements subjected to thermal gradients.

\subsection{Research Scope}

As previously discussed, the thermal compatibility analysis will include a linear elastic as well as finite element analysis of stresses and crack development occurring as a consequence of thermal loading. The linear elastic analysis will be based on expressions derived by Rahman et al. (1995) to study uncracked sections and expressions derived by Nanni et al. (2001) to study cracked sections. The finite element analysis will be performed on the basis of software called ADINA, in which a concrete model with non-linear capabilities is incorporated. The finite element method will, in addition, allow the project to consider several different geometries most likely found in practice.

The experimental program will consider CFRP and GFRP as prestressing reinforcement. To measure the effect of thermal action on bond strength of such reinforcement in concrete, beams will be tested in flexure with the load placed within the development length region. Any reduction in the load initiating slip of the reinforcement within the weathered specimens will be associated with thermal incompatibility of FRP reinforcement in concrete. Thermal cycles subjected to the beams will range between $-40^{\circ} \mathrm{C}$ and $+40^{\circ} \mathrm{C}$, which are typical extremes of temperature expected in the Canadian climate. 


\section{Chapter 2}

\section{Material Properties}

\subsection{Thermal Compatibility Analysis}

In light of achieving suitable results from analyses conducted on reinforced concrete elements, properties of concrete and reinforcement must be adequately represented. Material properties used in the linear elastic and finite element analyses will therefore be fully described and summarized in the several sections that follow.

\subsubsection{Concrete}

Concrete is a composite material composed of aggregates chemically bound together by hydrated cement. Although heterogeneous, the composition of concrete does not change with orientation causing material properties to have small and negligible directional dependence. The tensile strength, CTE and modulus of elasticity of concrete are the main properties that affect stresses generated around FRP reinforcing bars subjected to environmental temperature changes.

\subsubsection{Tensile Strength}

The tensile strength at which concrete cracks depends on a number of factors. These factors include the type of aggregate, the compressive strength of concrete as well as the presence of 
compressive stresses perpendicular to tensile stresses. Moreover, the actual value defining tensile strength strongly relies on the type of test carried out on samples.

There are two widely used tests, the first of which is called the modulus of rupture test. in this test, a plain concrete beam is subjected to flexure under third point loading until failure due to cracking occurs on the tension face. The second test is called the split cylinder test in which a concrete cylinder is placed on its side and loaded in compression. Specimens tested using the split cylinder test fail by splitting with a single fracture plane developing under the point of load. Both methods produce distinct stress fields within the test specimens and therefore generate different strengths. The tensile strength should therefore be chosen as that from a test method generating a similar stress state as that expected for the intended analysis. Since circumferential stresses are expected in concrete surrounding swelling FRP reinforcement, the tensile strength was chosen as that obtained from a double punch test and written in terms of the compressive strength of concrete $f_{c}^{\prime}$. Collins and Mitchell (1990) give approximate relationships that can be used to correlate tensile strength values obtained from various test methods, which include the double punch test. From these relationships, the tensile stress perpendicular to a failure plane developing during a double punch test $f_{d p}$ can be determined.

$f_{d p}=0.47 \sqrt{f^{\prime}}$

EQ.2.1

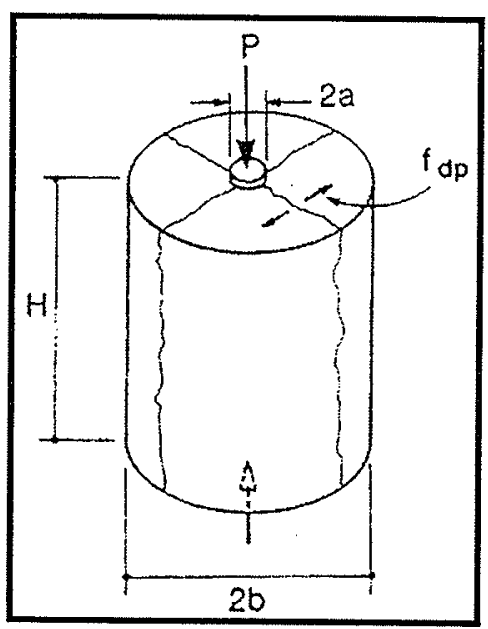

Figure 2.1 - Double Punch Test Layout 
As illustrated in Figure 2.1, the test consists of vertically placing a concrete cylinder between the loading platens of a uniaxial compression machine and applying compression through the use of two steel circular wedges located concentrically on the top and bottom surfaces of the cylinder. The cylinder normally fails by tensile splitting along several vertical planes traversing the axis of symmetry.

\subsubsection{Modulus of Elasticity}

Being affected by a variety of different parameters, the modulus of elasticity of concrete is a difficult property to estimate. This material property depends on the modulus of elasticity of the aggregate as well as that of the cement paste. It is further affected by the aggregate fraction in the mix as well as the loading rate. For this reason, equations for the modulus of elasticity presented in CSA A23.3 (1994) are formulated to adequately represent concrete originating from Canada and tested in accordance with relevant ASTM standard methods. Clause 8.6.2.2 of CSA A23.3 (1994) presents an expression derived for concrete with a density $\gamma_{c}$ ranging between 1,500 and $2,500 \mathrm{~kg} / \mathrm{m}^{3}$.

$$
E_{c}=\left(3300 \sqrt{f^{\prime}{ }_{c}}+6900\right)\left(\frac{\gamma_{c}}{2300}\right)^{1.5} \quad \text { EQ.2.2 }
$$

This equation corresponds to the secant modulus of elasticity at approximately $45 \%$ of the compressive strength. For reinforced concrete elements, the density used in this equation is normally taken as $2,300 \mathrm{~kg} / \mathrm{m}^{3}$ and corresponds to that of concrete without consideration of the reinforcement. For normal density concrete with compressive strengths ranging from 20 to 40 MPa, Clause 8.6.2.3 of CSA A23.3 (1994) allows the use of a different and simplified expression.

$$
E_{c}=4500 \sqrt{f_{c}^{\prime}}
$$


Values of the modulus of elasticity for compressive strengths varying between 20 and $90 \mathrm{MPa}$ were calculated and illustrated in Figure 2.2. Values in this figure were used in both analyses for the corresponding compressive strengths.

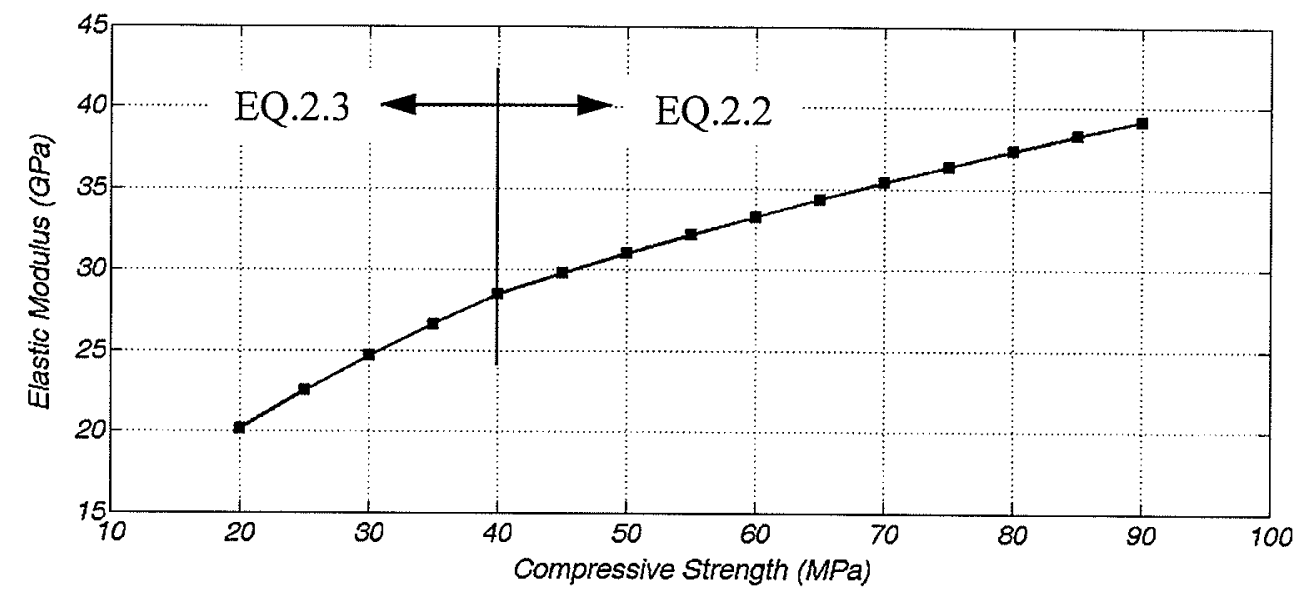

Figure 2.2 - Concrete Elastic Modulus $\left(\gamma_{c}=2300 \mathrm{~kg} / \mathrm{m}^{3}\right)$

\subsubsection{Coefficient of Thermal Expansion and Poisson's Ratio}

The CTE of concrete greatly depends on the type of aggregate and cement paste used in the mix. However, many sources commonly agree that ranges for normal density concrete reside between 9 and $13 \mu \varepsilon /{ }^{\circ} \mathrm{C}$ when made with siliceous aggregates and between 6 and $9 \mu \varepsilon /{ }^{\circ} \mathrm{C}$ when made with calcareous aggregates. A value of $10 \mu \varepsilon /{ }^{\circ} \mathrm{C}$ is therefore typically used for plain concrete.

Below critical stresses causing unstable crack propagation within concrete in compression, Poisson's ratio $v_{c}$ can vary between 0.11 and 0.21 and is more likely to fall within the range of 0.15 and 0.20. Based on biaxial tests conducted on concrete, Kupfer et al. (1969) reported values for Poisson's ratio to range between 0.18 and 0.20 for concrete loaded in compression and tension. Since thermal expansion of FRP reinforcement is expected to cause a combination of radial pressure and circumferential tensile stresses in the surrounding concrete, the value of 
Poisson's ratio was selected as 0.19 for a better representation of values stated by Kupfer et al. (1969).

\subsubsection{FRP Reinforcement}

One of the similarities between concrete and FRP reinforcement is that they are both composite materials. However, the composition of FRP reinforcing bars consists of longitudinal fibres embedded in a resin matrix, which causes properties in the longitudinal and transverse directions to appreciably differ. More specifically, properties in the longitudinal direction will be significantly influenced by the presence of fibres while the resin will have a considerable effect on those in the transverse direction. The basic properties of reinforcement affecting stresses occurring in the neighbouring concrete include the modulus of elasticity and CTE in the transverse direction.

The modulus of elasticity and CTE in the longitudinal direction will also affect stresses within the concrete cover but at a lesser extent. In addition, these values can easily be obtained experimentally and are readily available from the manufacturer or literature for various types of FRP reinforcement. Unfortunately, the lack of standardized methods for reinforcing bars causes very little to be known about Poisson's ratio and these properties in the transverse direction.

\subsubsection{Transverse Modulus of Elasticity}

The transverse modulus of elasticity of FRP reinforcement is much smaller than in the longitudinal direction due to fibres being aligned along the bar axis. Although theoretical expressions exist for this property (Jones 1975 and Chawla 1987), more realistic values have been obtained experimentally at room temperatures (Rahman et al., 1995). Experimental values were shown to be considerably lower than theoretical estimates and comparable to that of the

resin. For this reason, values of the transverse modulus of elasticity for the analyses carried out will be taken as the typical values for resin, which range from 2,000 to $4,000 \mathrm{MPa}$. 


\subsubsection{Transverse Coefficient of Thermal Expansion}

Due to the little moderating effect of fibres in the transverse direction, the transverse CTE of FRP reinforcement will be mainly affected by substantial swelling of the resin under thermal action. As a result, the transverse CTE will be significantly higher than in the longitudinal direction. However, the moderating effect of fibres and consequent restraint on thermal expansion in the longitudinal direction can cause the transverse CTE of FRP reinforcement to reach values exceeding those of the resin (Agarwal et al., 1990). It was found that the CTE of neat thermosetting resins such as polyesters, vinylesters and epoxies usually ranges between 40 and $90 \mu \varepsilon /{ }^{\circ} \mathrm{C}$ (Schapery, 1968). In order to represent the wide range of commercially available FRP reinforcing bars, values for the transverse thermal expansion coefficient used in the analyses were chosen to reside between 20 and $110 \mu \varepsilon /{ }^{\circ} \mathrm{C}$.

\subsubsection{Poisson's Ratio}

There are a total of three independent Poisson ratios arising from the composition of FRP reinforcement. The ratios depend on the direction of loading and are designated $v_{f}^{T L}, v_{f}^{L T}$ and $v_{f}^{T}$ where the first superscript denotes the direction of loading while the second that of lateral strain ( $T$ - transverse, $L$ - longitudinal). Only the last two ratios are required for the analyses presented in this thesis. Being less critical than the elastic modulus and CTE on stresses within the concrete cover, theoretical estimates similar to those determined by Rahman et al. (1995) were chosen to be sufficiently accurate. These estimates correspond to 0.3 for $v_{f}^{L T}$ and 0.35 for $v_{f}^{T}$. 


\subsection{Experimental Program}

\subsubsection{FRP Reinforcement}

As mentioned in earlier sections, concrete beams in this research will be prestressed with GFRP and CFRP tendons. These tendons are manufactured by Hughes Brothers and respectively carry the designation of Aslan 100 and Aslan 200. Table 2.1 lists the specifications provided by the manufacturer for the nominal bar diameter of $9 \mathrm{~mm}$ used in this project.

To confirm tensile properties provided by the manufacturer, a total of four tension tests were conducted at the University of Manitoba for each type of reinforcement. Annex B of CSA S806 (2002) was used to establish requirements for testing specimens of FRP reinforcement in tension.

Table 2.1 - Reinforcement Specifications (Hughes Brothers, Inc.)

\begin{tabular}{|l|c|c|}
\hline \multicolumn{1}{|c|}{ PARAMETER } & CFRP (Aslan 200) & GFRP (Aslan 100) \\
\hline Nominal Diameter $(\mathrm{mm})$ & 9 & 9.53 \\
\hline Sectional Area $\left(\mathrm{mm}^{2}\right)$ & 65.2 & 84.3 \\
\hline Ultimate Strength in Tension $(\mathrm{MPa})$ & 2,068 & 760 \\
\hline Elastic Modulus $(\mathrm{MPa})$ & 124,000 & 40,800 \\
\hline Ultimate Strain $(\mu \varepsilon)$ & 16,677 & 18,627 \\
\hline Transverse CTE $\left(\mu \varepsilon /{ }^{\circ} \mathrm{C}\right)$ & 74 to 104 & 21 to 23 \\
\hline Longitudinal CTE $\left(\mu \varepsilon /{ }^{\circ} \mathrm{C}\right)$ & -4 to 0 & 6 to 10 \\
\hline Maximum bond Stress $(\mathrm{MPa})$ & 8.45 & 11.6 \\
\hline
\end{tabular}

\subsubsection{Tension Tests}

For the purpose of applying tension to the specimens, steel anchors adapted to fit the grips of the testing machine were bonded to the ends of the FRP reinforcement. The anchors chosen for this 
study were high-pressure steel pipes commonly used in plumbing applications. Geometric characteristics of these anchors are in accordance with Annex B of CSA S806 (2002) and are illustrated in Figure 2.3.

A West System 105/205 two-part low viscosity epoxy was used to bond anchors to GFRP bars while a Bristar 100 expansive grout was used to cement anchors at the ends of CFRP bars. The interior wall of each anchor was cleaned using a sand blaster. A washer was glued with Devcon 5 -minute ${ }^{\circledR}$ epoxy gel at the bottom of each anchor to prevent leakage of the bonding agent. Anchors were subsequently cast in a vertical position using a suitable jig to maintain axial alignment with the FRP reinforcement. Specimens were kept in their vertical positions at least 12 hours and tested more than 24 hours after casting.

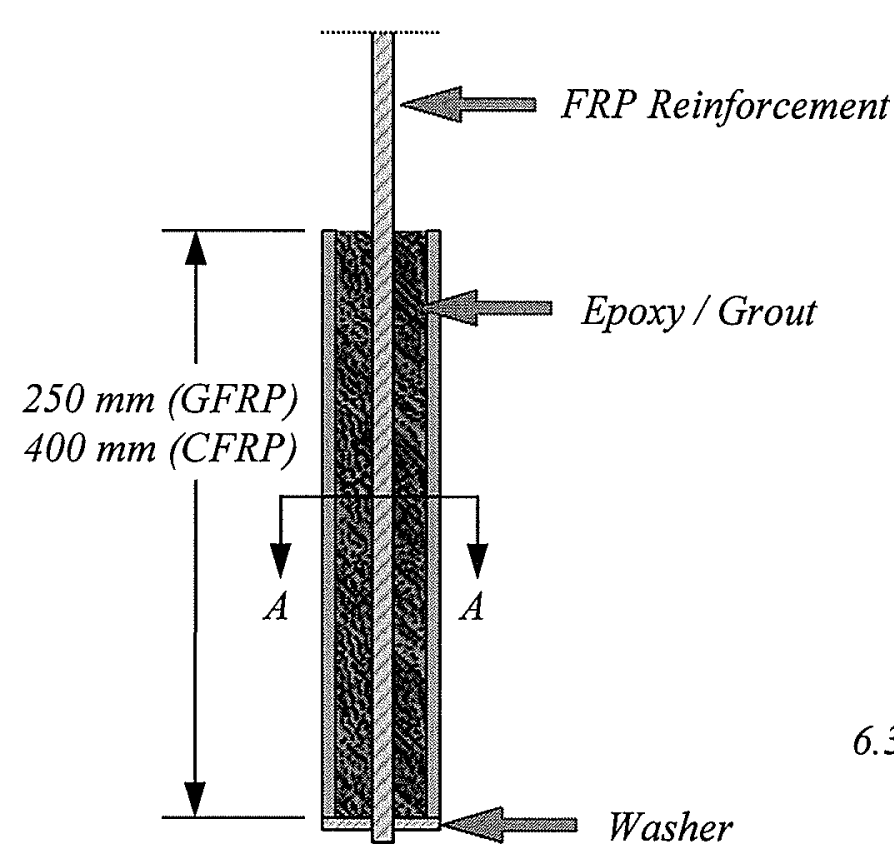

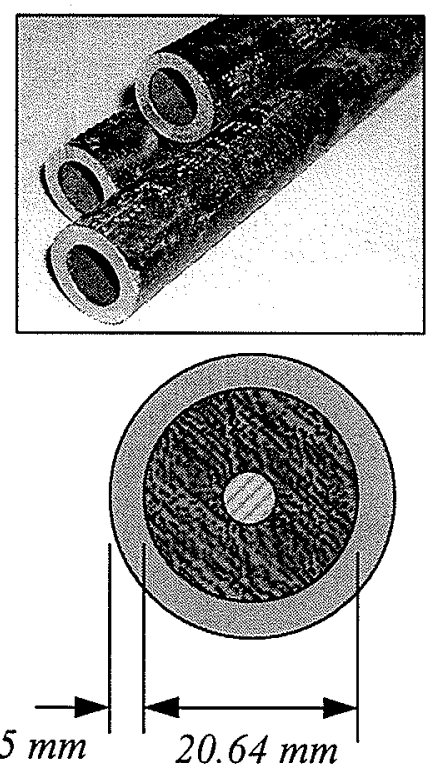

$\underline{\text { Section } A-A}$

Figure 2.3 - Anchor Geometric Properties

A testing machine with loading capacity of 60 kips $(267 \mathrm{kN})$ was used for all tension tests. Specimens were instrumented with a longitudinal strain gauge to determine the modulus of elasticity while two GFRP and CFRP specimens were instrumented with transverse strain gauges 
to evaluate Poisson's ratio. Load and strain was monitored and recorded using a 32 Channel Data Acquisition (DAQ) System. The testing layout is illustrated in Figure 2.4.

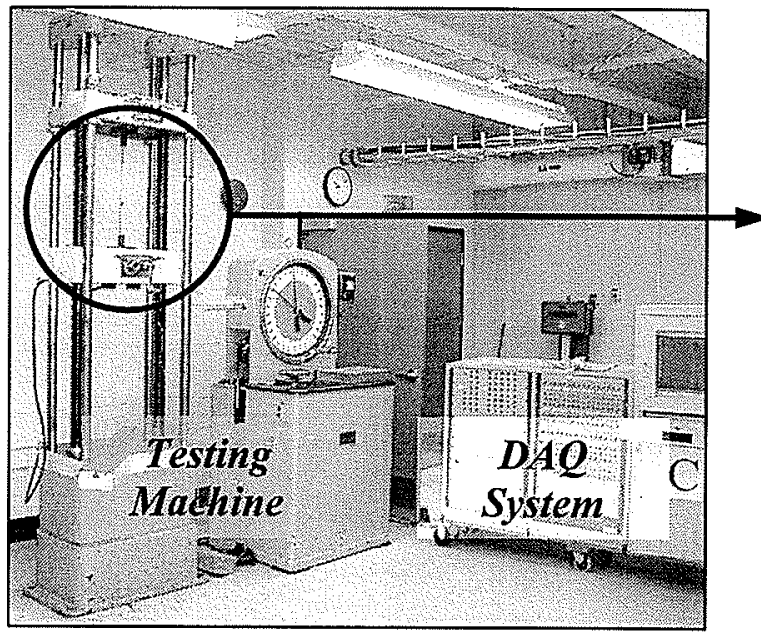

(a) 60 kip Testing Machine and DAQ System

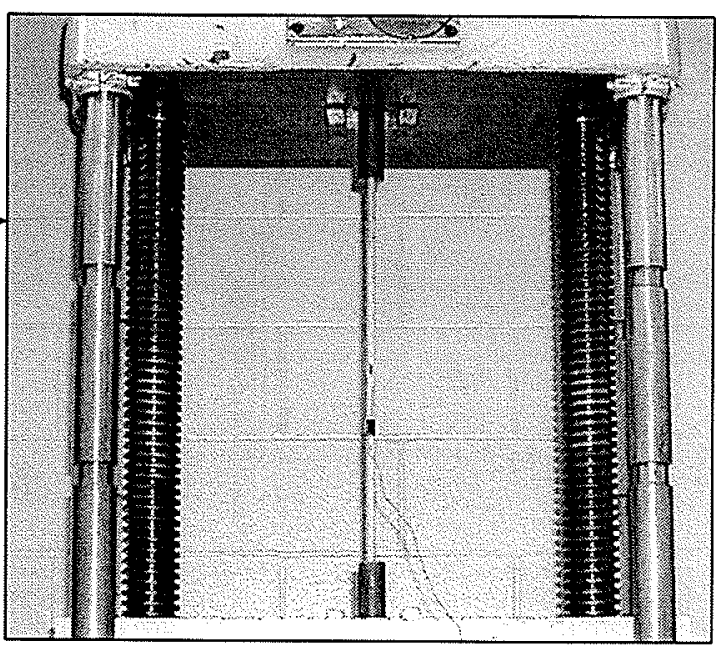

(b) Positioned Specimen

Figure 2.4 - Testing Layout for Tension Tests

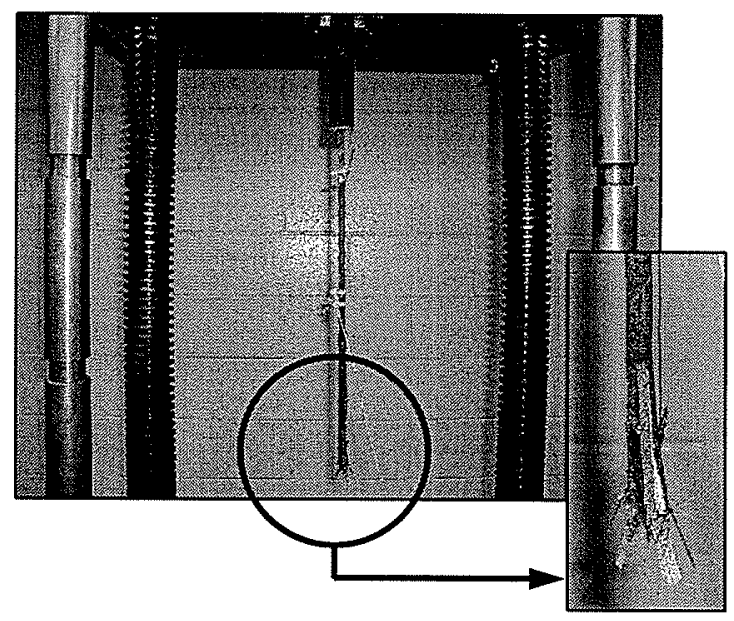

(a) CFRP Specimen

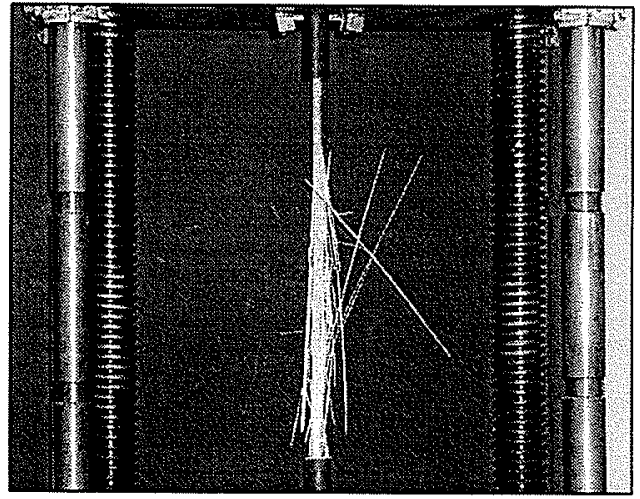

(b) GFRP Specimen

Figure 2.5 - FRP Specimen Failure Characteristics

All failures initiated with the sound of individual fibres fracturing followed by complete rupture of the specimen within the gauge length portion away from the anchors. In opposition to that observed for CFRP specimens, fibres in the GFRP specimens were found to separate and open 
out at failure. Failure characteristics of both tendons are illustrated in Figure 2.5 while results from the tension tests are summarized in Table 2.2. In addition, Figure 2.6 shows the stressstrain relationships obtained from typical tension tests conducted on CFRP and GFRP reinforcing bars. The results emphasize linear elastic behaviour of FRP reinforcement to failure.

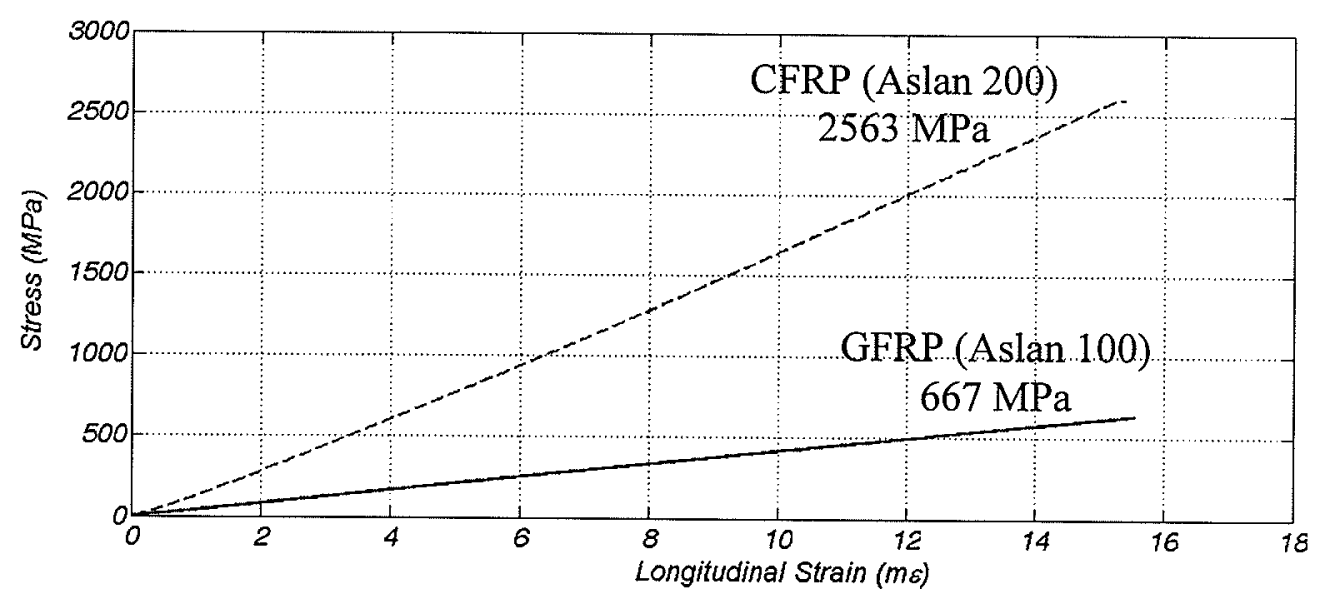

Figure 2.6 - Stress-Strain Relationship of CFRP and GFRP Reinforcement Tested

Table 2.2 - Tension Test Results

\begin{tabular}{|l|c|c|}
\hline \multicolumn{1}{|c|}{ PARAMETER } & CFRP (Aslan 200) & GFRP (Aslan 100) \\
\hline Ultimate Strength in Tension (MPa) & 2,563 & 667 \\
\hline Elastic Modulus (MPa) & 171,962 & 39,081 \\
\hline Ultimate Strain $(\mu \varepsilon)$ & 15,337 & 17,310 \\
\hline Poisson's Ratio $v_{f}^{L T}$ & -0.35 & -0.31 \\
\hline
\end{tabular}

\subsubsection{Concrete}

Concrete with a compressive strength of $50 \mathrm{MPa}$ was selected for the prestressed beams considered in this research. Two sets of beams were cast for the research. The first set (control beams) was used to investigate development length properties of the tendons and evaluate flexural behaviour. The second set (weathered beams) was used to evaluate bond strength after 
thermal cycling. To improve freeze-thaw resistance of concrete under cyclic temperature loading, air content in the range of 6 to 8 percent was requested for all beams in this project. The mix design is given in Table 2.3.

\subsubsection{Material Testing}

To determine mechanical properties of concrete used in this research, batches of cylindrical concrete specimens were cast simultaneously with the beams. Concrete cylinders were kept in the same curing environment as the prestressed elements at all times. The compressive strength and chord modulus of elasticity were determined through standard test methods in accordance with ASTM designations C39 (2004) and C469 (2002), respectively. In addition, the tensile splitting strength of cylindrical concrete specimens was determined on the basis of the standard test method described in ASTM designation C496 (2004).

Table 2.3 - Concrete Mix Design and Properties

\begin{tabular}{|l|c|}
\hline \multicolumn{1}{|c|}{ COMPONENT } & VALUE \\
\hline Cement & $407 \mathrm{~kg} / \mathrm{m}^{3}$ \\
\hline Classified Sand & $727 \mathrm{~kg} / \mathrm{m}^{3}$ \\
\hline Water & $147 \mathrm{~kg} / \mathrm{m}^{3}$ \\
\hline $10 \mathrm{~mm}$ Coarse Aggregate & $1120 \mathrm{~kg} / \mathrm{m}^{3}$ \\
\hline Water Reducing Admixtures & $2 \mathrm{~L}$ \\
\hline Air Entraining Agent & $33 \mathrm{ml}$ \\
\hline Water Cement Ratio & 0.361 \\
\hline Density of Concrete & $2401 \mathrm{~kg} / \mathrm{m}^{3}$ \\
\hline
\end{tabular}

Results from tests performed on concrete cylinders for the two sets of beams investigated in this project are listed in Table 2.4 and illustrated in Figure 2.7. To ensure sufficient strength was attained at critical stages during the curing process, compression and splitting tension tests were 
conducted at the time of prestress release and testing of the beams. For the weathered beams, concrete cylinders were placed within the chambers during thermal cycling. To gain awareness on the compressive strength gain under thermal action, additional compression tests were conducted immediately before as well as during the cycles. The concrete cylinder test layout is shown in Figure 2.8(a) for determination of elastic modulus and in Figure 2.8(b) for determination of compressive strength.

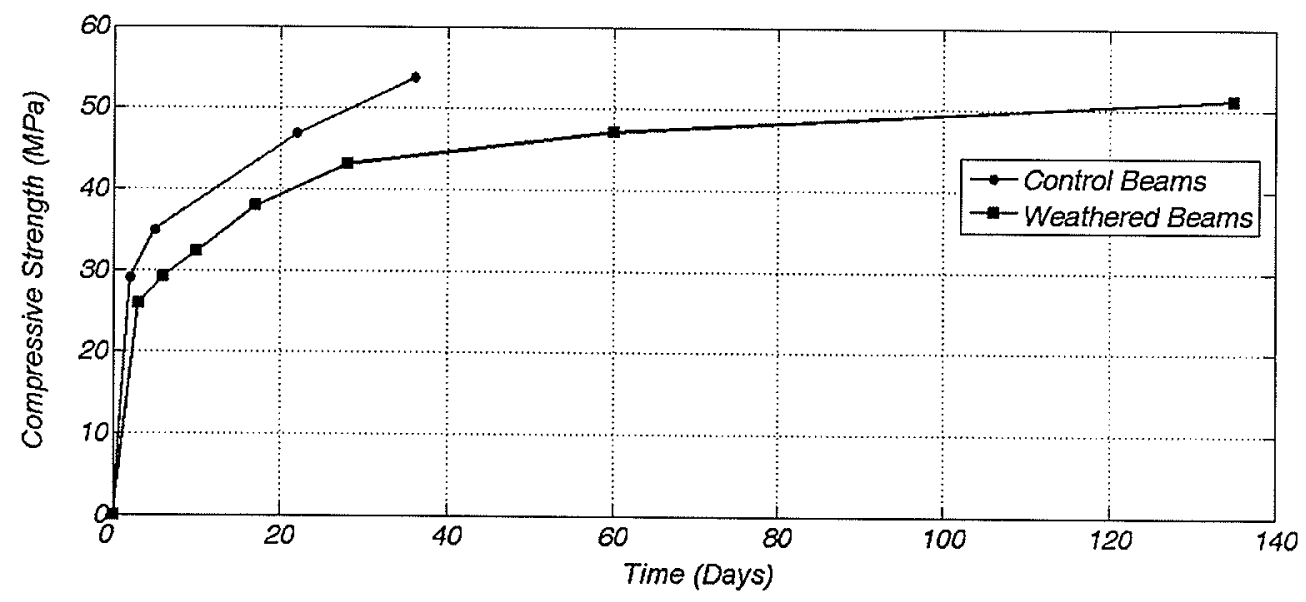

Figure 2.7 - Concrete Compression Test Results

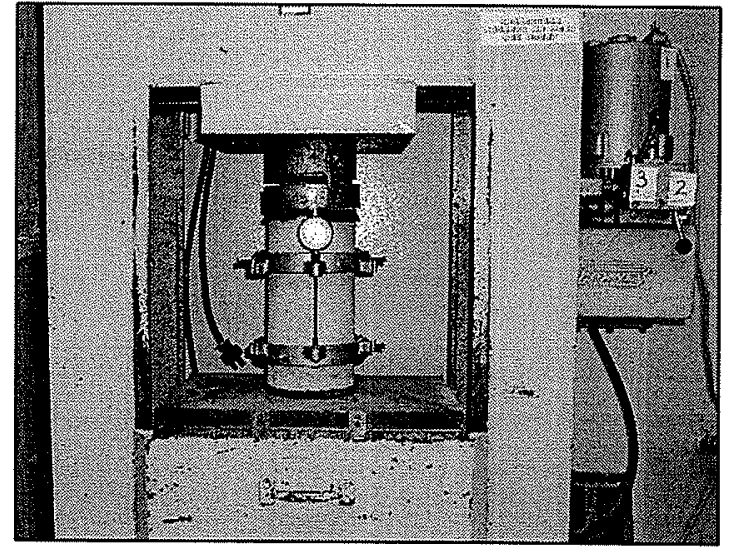

(a) Compressometer for Static Elastic Modulus Test

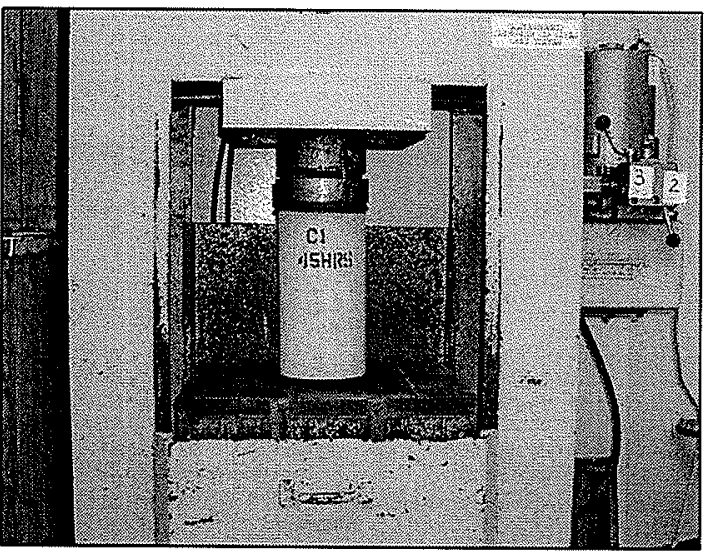

(b) Standard Compression Test

Figure 2.8 - Concrete Cylinder Tests

It is apparent from Figure 2.7 that the compressive strength obtained for concrete cast in specimens intended for the weathering process is lower than that obtained for concrete cast in the control specimens. Having been cast at Lafarge Canada, the beams intended for the 
weathering process were in a relatively dry environment. Although regularly soaked after casting, moisture loss during the first weekend was the main cause in the divergence of compressive strength results. Better supervision of the control beams cast at the University of Manitoba was possible during the curing process. The Structures Laboratory was accessed during the weekends to allow continuous moistening of the beams for superior compressive strength results.

Table 2.4 - Concrete Strength Summary

(a) Control Beams

\begin{tabular}{|c|c|c|}
\hline Time of Test (Days) & Compressive Strength (MPa) & Split Cylinder Strength (MPa) \\
\hline 2 & 29 & - \\
\hline 5 & 35 & 2.7 \\
\hline 22 (Release of Prestress) & 47 & 3.2 \\
\hline 36 (Flexural Test) & 54 & 3.7 \\
\hline
\end{tabular}

(b) Weathered Beams

\begin{tabular}{|c|c|c|}
\hline Time of Test (Days) & Compressive Strength (MPa) & Split Cylinder Strength (MPa) \\
\hline 3 & 26 & - \\
\hline 6 & 29 & 2.5 \\
\hline 10 & 32 & 2.8 \\
\hline 17 (Release of Prestress) & 38 & - \\
\hline 28 (Before Cycles) & 43 & - \\
\hline 60 (During Cycles) & 47 & 3.3 \\
\hline 135 (Flexural Bond Test) & 51 & -9 \\
\hline
\end{tabular}




\section{Chapter 3}

\section{Linear Elastic Analysis of Thermally Induced Stresses}

\subsection{Assumptions}

Several assumptions subsist for the linear elastic analysis presented in this thesis. These assumptions were made in the derivation of expressions defining the development of stresses and cracks within the cover of concrete elements reinforced with swelling FRP bars. The assumptions are listed below.

(1) The analysis is aimed at studying the development of cracking within the concrete cover of structural elements only. In other words, sufficient spacing is provided between individual bars to prevent overlap of stress fields that can greatly influence initiation and development of the splitting phenomenon within the concrete cover.

(2) The effect of transverse reinforcement for crack control and shear resistance is neglected. The analysis is thereby focused on evaluating the contribution of concrete alone to the effect of bursting stresses within the clear cover.

(3) Concrete and FRP material have a linear elastic behaviour until complete splitting of the clear cover. 


\subsection{Axisymmetric Model Representation of Composite Rod Embedded in Concrete}

Accounting for parameters and material properties discussed in the previous sections, numerous studies have attempted to describe the pressure and circumferential stresses induced by thermal swelling of FRP reinforcement. All derivations found in the literature were based on the closedform two-dimensional axisymmetric solution of a round rod embedded in a cylinder of a second material with different thermo elastic constants presented by Timoshenko and Goodier (1970). Modifying this solution for the case of a unidirectional composite rod in a concrete cylinder, researchers are able to predict whether swelling of composite reinforcement under moderate temperature increases would be sufficient to bring the concrete to its tensile strength and instigate splitting failure.

Figure 3.1 illustrates crack development in the concrete cover of a structural element with sufficient bar spacing and illustrates the use of an axisymmetric concrete cylinder as a model in the linear elastic analysis. The concrete cylinder in this figure describes the localized zone influenced by transverse swelling of the reinforcement with the thickness representing the clear cover provided.

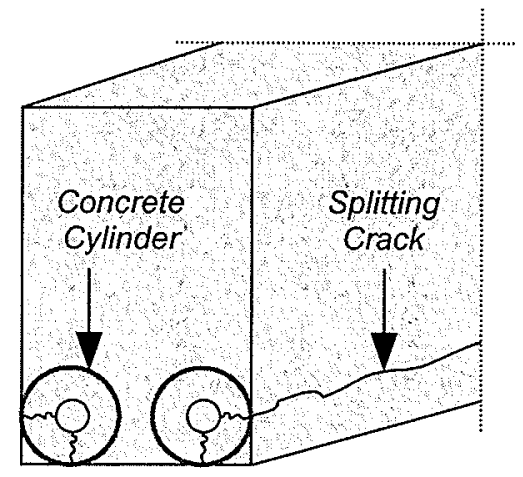

(a) Crack Development Within Cover

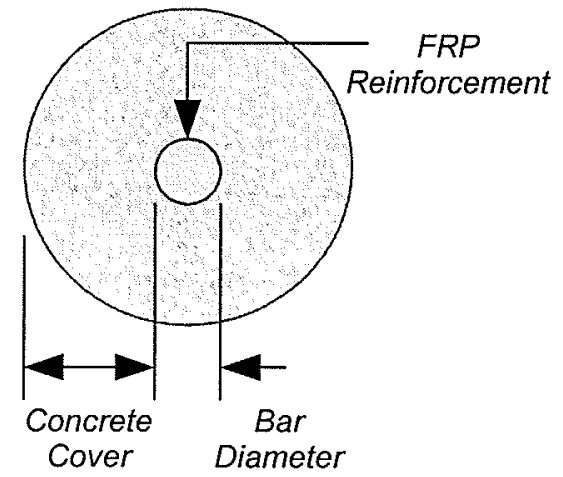

(b) Concrete Cylinder Model

Figure 3.1 - Splitting Failure Surfaces and Model Representation 
Additional sources of circumferential stresses will also be considered in the analysis and discussed in the sections that follow. These sources include the requirement of axial compatibility under thermal loading as well as the Hoyer effect in the transfer region of prestressed elements.

\subsection{Stress Distribution Within Concrete Cover}

Polar coordinates extending in the radial, circumferential and axial directions are convenient for describing axisymmetric geometries as the one illustrated in Figure 3.1(b). Figure 3.2 depicts the polar coordinate system used in the linear elastic analysis to describe the geometry and behaviour of axisymmetric elements within the linear elastic model. The stresses relevant to the analysis are also illustrated in the figure.

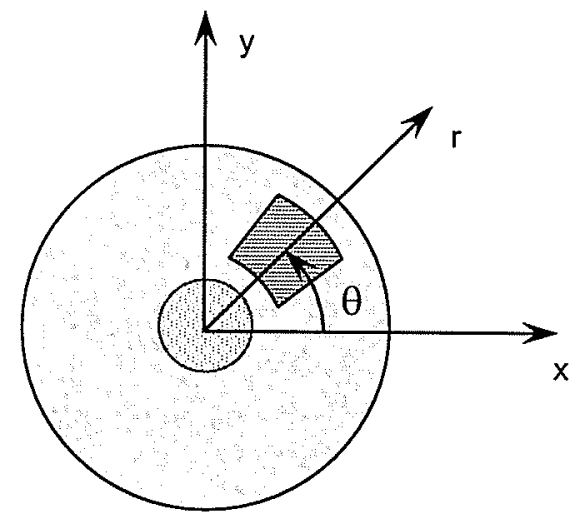

(a) Polar Coordinate System

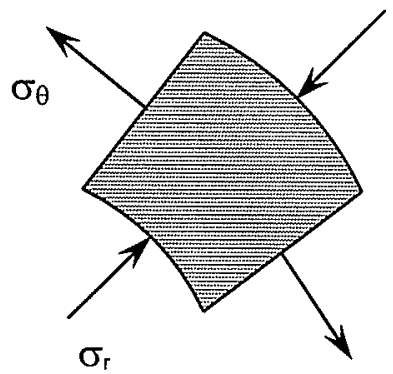

(b) State of Stress

Figure 3.2 - Composite Rod in Concrete Cylinder

It should be emphasized at this point that there are no shear strains and shear stresses acting in the radial and circumferential directions of axisymmetric elements. Consequently, stresses act normal to the element faces only and are considered principal stresses. Cracks will therefore propagate perpendicular to the orientation of circumferential stresses when the concrete reaches its tensile strength. 
Radial (EQ.3.1) and circumferential (EQ.3.2) stresses within the concrete cover of the linear elastic model were derived for the case of a thick walled cylinder subjected to internal pressure. These stresses are a function of the pressure $p$ exerted by the reinforcement on the concrete as well as the radius of the linear elastic model $b$ and bar diameter $a$. They are inversely proportional to the radial distance $r$ away from the centre of the reinforcement, which causes stresses at the interface between the materials to be higher.

$$
\begin{gathered}
\sigma_{r}=\frac{a^{2}}{b^{2}-a^{2}}\left(1-\frac{b^{2}}{r^{2}}\right) p \\
\sigma_{\theta}=\frac{a^{2}}{b^{2}-a^{2}}\left(1+\frac{b^{2}}{r^{2}}\right) p
\end{gathered}
$$

It should be noticed that the sum of the stresses defined in these equations is constant through the thickness of the cylinder. Consequently, stresses will generate a uniform extension and contraction on every element in the axial direction such that a cross section perpendicular to the axis remains plane whenever internal pressure is applied to the cylinder. For this reason, deformations in an element of the cylinder will not interfere with the deformation of adjacent elements. The consideration of plane stress for the model is thereby justified by making the additional assumption that the ends of the cylinder are unrestrained.

\subsection{Uniform Internal Pressure}

The differential pressure generated across the boundary separating the FRP reinforcement and concrete is derived considering compatibility of radial displacement between the two materials when subjected to a temperature fluctuation $\Delta T$. 
$p=\frac{\varepsilon^{T}}{\left(\beta+v_{c}\right) / E_{c}+\left(1-v_{f}^{T T}\right) / E_{f}^{T}} \quad$ EQ.3.3

$\beta=\frac{b^{2}+a^{2}}{b^{2}-a^{2}}$

EQ.3.4

Here, $p$ is the uniform internal pressure at the interface of the two materials caused by differential swelling of the reinforcement, $\varepsilon_{T}$ is the transverse differential strain between the materials causing internal pressure, $E_{f}^{T}$ is the transverse modulus of elasticity of reinforcement, $v_{f}^{T}$ is Poisson's ratio of the reinforcement describing transverse strains due to loading in the same orientation and $v_{c}$ is Poisson's ratio for concrete.

\subsection{Transverse Differential Strain}

This thesis presents methods to account for three sources of transverse differential strain used in the calculation of internal pressure. These sources are fully discussed in the sections that follow and include:

(1) Incompatibility of thermal expansion between materials in the transverse direction.

(2) Incompatibility of thermal expansion between materials in the longitudinal direction.

(3) Hoyer effect in the transfer region of prestressed structural elements.

\subsubsection{Transverse Thermal Incompatibility}

Under the influence of temperature fluctuations $\Delta T$, both materials will undergo length changes in the transverse direction. The two following equations define unrestrained transverse thermal strain arising from this change in length for the FRP reinforcement and concrete material, respectively. 


$$
\begin{array}{ll}
\varepsilon_{f}^{T}=\alpha_{f}^{T} \Delta T & \mathrm{EQ} .3 .5 \\
\varepsilon_{c}^{T}=\alpha_{c} \Delta T & \mathrm{EQ} 3.6
\end{array}
$$

It should be emphasized that the surrounding concrete will restrain transverse strains in the FRP reinforcement when incompatibility between the materials exists. When the transverse CTEs differ, this restraint will contribute to the transverse differential strain and is equivalent to the difference between the unrestrained transverse thermal strains of both materials.

$$
\varepsilon_{1}^{T}=\left(\alpha_{f}^{T}-\alpha_{c}\right) \Delta T \quad \text { EQ.3.7 }
$$

\subsubsection{Longitudinal Thermal Incompatibility - Compatibility of Axial Deformation}

Longitudinal thermal incompatibility occurs when the longitudinal CTE of each material differs. This incompatibility causes additional transverse differential by the compatibility requirement of axial deformations when the bond is preserved between the two materials.

This requirement will add a mechanical strain to the unrestrained longitudinal thermal strain of each material. Since this thermal strain is assumed to take place independently of any axial loads, we can account for it by modifying Hooke's law for one-dimensional mechanical strain.

$\varepsilon=\frac{\sigma}{E}+\alpha \Delta T \quad \quad \mathrm{EQ} .3 .8$

In order to calculate the longitudinal strain defined by Equation 3.8, it is necessary to elaborate the concept of compatibility in the axial direction. As illustrated in Figure 3.3, the requirement of compatibility will cause axial forces to develop in both materials when different thermal properties exist. For the system to be in equilibrium, the sum of these forces should equal zero. 

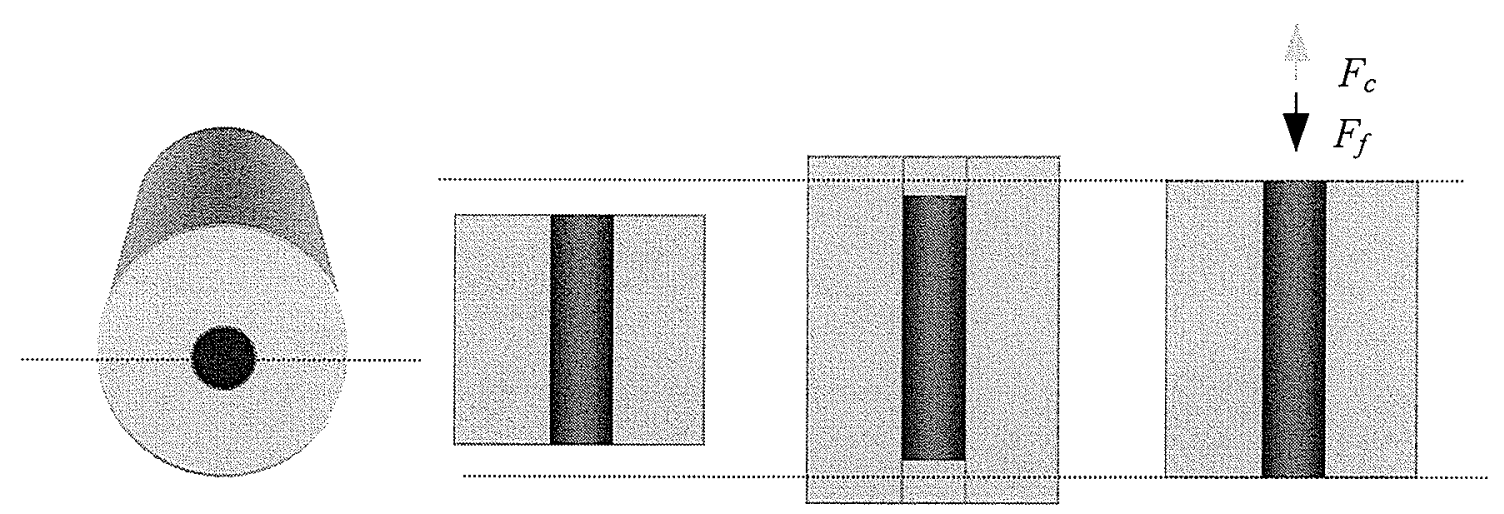

Original Shape

Free Thermal Expansion of Both Materials

Requirement of

Compatibility

Figure 3.3 - Thermal Expansion, Compatibility of Axial Deformation and Force Equilibrium

Writing Equation 3.8 in terms of axial stresses $\sigma$ and substituting the cross-sectional area as well as the mechanical and physical properties of individual materials, we can obtain equations corresponding to the axial forces in the reinforcement (EQ.3.11) and concrete cylinder (EQ.3.12).

$\sigma=E \varepsilon-E \alpha \Delta T$

EQ.3.10

$F_{f}=\frac{A_{f} E_{f}^{L}}{L} u_{f}-A_{f} E_{f}^{L} \alpha_{f}^{L} \Delta T$

$F_{c}=\frac{A_{c} E_{c}}{L} u_{c}-A_{c} E_{c} \alpha_{c} \Delta T$

Here, $A_{f}$ is the cross-sectional area of reinforcement, $A_{c}$ is the cross-sectional area of concrete cylinder, $E_{f}^{L}$ is the longitudinal elastic modulus of reinforcement, $E_{c}$ is the elastic modulus of concrete and $\alpha_{f}^{L}$ is the longitudinal CTE of reinforcement. Having derived the axial forces in each material and recalling that they should maintain equilibrium, we can solve for the increase in 
length of the system due to thermal loading, $u$. The requirement of compatibility will cause this increase in length to be the same in both materials $\left(u_{f}[\mathrm{FRP}]=u_{c}[\right.$ CONCRETE $\left.]=u\right)$.

$$
u=\frac{A_{c} E_{c} \alpha_{c} \Delta T+A_{f} E_{f}^{L} \alpha_{f}^{L} \Delta T}{A_{c} E_{c}+A_{f} E_{f}^{L}} L
$$

The difference between the increase in length defined by the previous equation and the unrestrained thermal expansion of the reinforcement (EQ.3.14) and concrete cylinder (EQ.3.15) represents the axial deformation that contributes to transverse differential strain for both materials. Equations 3.16 and 3.17 define the axial contribution from the reinforcement and concrete, respectively.

$$
\delta_{f}^{L}=\alpha_{f}^{L} L \Delta T
$$

$\delta_{c}^{L}=\alpha_{c} L \Delta T$

EQ.3.15

$$
\begin{array}{ll}
\Delta \delta_{f}^{L}=\frac{A_{c} E_{c}}{A_{c} E_{c}+A_{f} E_{f}^{L}}\left(\alpha_{f}^{L}-\alpha_{c}\right) L \Delta T & \text { EQ.3.16 } \\
\Delta \delta_{c}^{L}=\frac{A_{f} E_{f}^{L}}{A_{c} E_{c}+A_{f} E_{f}^{L}}\left(\alpha_{c}-\alpha_{f}^{L}\right) L \Delta T & \text { EQ.3.17 }
\end{array}
$$

Finally, the additional transverse differential strain caused by the requirement of axial compatibility is obtained by multiplying the axial contribution strains with the appropriate Poisson's ratio of each material (EQ.3.17). In this equation, $\nu^{L T}$, is Poisson's ratio of the reinforcement describing lateral strains arising from loading in the axial direction.

$\varepsilon_{2}^{T}=\frac{\left(v_{f}^{L T} \Delta \delta_{f}+v_{c} \Delta \delta_{c}\right)}{L}$

EQ.3.18 


\subsubsection{Hoyer Effect}

It is well known that the prestressing force cannot be fully developed at the edges of concrete elements and that a transfer length is required. For this reason, the full prestressing force will be released at the edges of prestressed elements causing the occurrence of splitting stresses. This phenomenon is called the Hoyer effect and is described by the following equation.

$\varepsilon_{3}^{T}=\nu_{f}^{L T} \varepsilon_{p o}$

EQ.3.19

Where $\varepsilon_{p o}$ is the initial prestressing strain submitted to the reinforcement and $\varepsilon_{3}{ }^{T}$ is the additional transverse differential due to the full prestressing force being released at the edge of prestressed elements. Lastly, the total transverse differential strain $\varepsilon^{T}$ used in the calculation of pressure can be written as a combination of all contributions from transverse and longitudinal thermal incompatibilities as well as the Hoyer effect.

$\varepsilon^{T}=\varepsilon_{1}^{T}+\varepsilon_{2}^{T}+\varepsilon_{3}^{T} \quad$ EQ.3.20

\subsubsection{Equivalent Temperature Change}

The total differential strain defined by equation 3.20 can be converted to an equivalent temperature change. This temperature change is useful when using charts that define the maximum temperature change that can be subjected to an element without cracking or splitting of the concrete cover. In this way, designers and researchers can estimate the extent of damage caused by a combination of thermal action, axial compatibility and Hoyer effect. 
The equivalent temperature change can be calculated by dividing the total transverse differential by the transverse disparity of thermal expansion coefficients.

$$
\Delta T_{\text {equivalent }}=\frac{\varepsilon^{T}}{\left(\alpha_{f}^{T}-\alpha_{c}\right)}
$$

\subsection{Post-Cracking Analysis}

When circumferential stresses reach the tensile strength of concrete, a cracked zone forms around the reinforcement as illustrated in Figure 3.3. At this point, stresses in the uncracked region are calculated on the basis of different expressions. These stresses depend on the radius of the cracked region $r_{c r}$ and the pressure $p^{\prime}(E Q .3 .23)$ exerted on the uncracked region.

$$
\sigma_{\theta}^{\prime}=\frac{a^{2}}{b^{2}-a^{2}}\left(1+\frac{b^{2}}{r^{2}}\right) \frac{a}{r_{c r}} p^{\prime}
$$

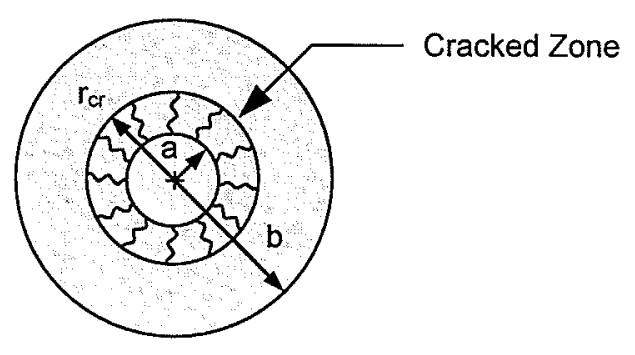

Figure 3.3 - Development of Splitting Within Concrete Cover

$$
\begin{aligned}
& p^{\prime}=\frac{\varepsilon^{T}}{\left(\ln (\chi)+\beta^{\prime}+\nu_{c}\right) / E_{c}+\left(1-\nu_{f}^{T T}\right) / E_{f}^{T}} \\
& \chi=\frac{r_{c r}}{a}
\end{aligned}
$$

EQ.3.24 


$$
\beta^{\prime}=\frac{b^{2}+r_{c r}{ }^{2}}{b^{2}-r_{c r}{ }^{2}}
$$

The radius of the cracked region can be determined by noticing that circumferential stresses at the tip of a crack and interface between the cracked and uncracked regions are equal to the tensile strength of concrete $f_{t}$. Setting $r=r_{c r}$ and $\sigma_{\theta}{ }_{\theta}=f_{r}$ in Equation 3.22 defines the roots of a third degree polynomial (EQ.3.26).

$$
\begin{aligned}
& \psi^{3}+A \psi^{2}-\psi+A=0 \\
& A=\frac{p^{\prime} a}{f_{t} b} \\
& \psi=\frac{r_{c r}}{b}
\end{aligned}
$$

As examined by Aiello et al. (2001), this third degree polynomial has only one meaningful solution for the actual problem when $A$ ranges from 0 to 0.3 . Absence of a solution occurs for values of $A$ beyond 0.3 where too high a radial compression develops or too small a concrete cover for the uncracked circular crown of concrete exists to prevent the splitting phenomenon from occurring. Consequently, the maximum radial compression that can be held by the concrete (EQ.3.29) and the corresponding outer radius of the cracked circular crown (EQ.3.30) for a given geometry and a given set of material properties can be determined when $A$ reaches a value of 0.3 .

$$
\begin{aligned}
& p_{\text {max }}^{\prime}=0.3 \frac{f_{t} b}{a} \\
& r_{c r}=0.48 b
\end{aligned}
$$

EQ.3.30 


\subsection{Uncracked Section Analysis Results}

As previously discussed, radial and circumferential stresses develop within the concrete cover of the linear elastic model. While radial stresses are compressive, circumferential stresses will be tensile and cause instigation of cracking within the concrete cover. To investigate this aspect of the analysis, the distribution of circumferential stresses is illustrated in Figure 3.4 for concrete compressive strengths ranging from 30 to $90 \mathrm{MPa}$ and a transverse elastic modulus for the reinforcement of 3,000 $\mathrm{MPa}$. To demonstrate the effect of increasing the concrete cover, temperature fluctuations used for these distributions were chosen to represent those causing initiation of cracking within the smallest concrete cover to bar diameter (c/d) ratio considered in the analysis.

As expected from expressions defining stresses within the cover of the linear elastic model, Figure 3.4 confirms that circumferential stresses attain their highest level at the interface between the materials and will cause cracking to initiate at this location. The expressions also cause these stresses to dissipate rapidly within the cover at a rate proportional to the cube of the radial distance $r(E Q .3 .31)$.

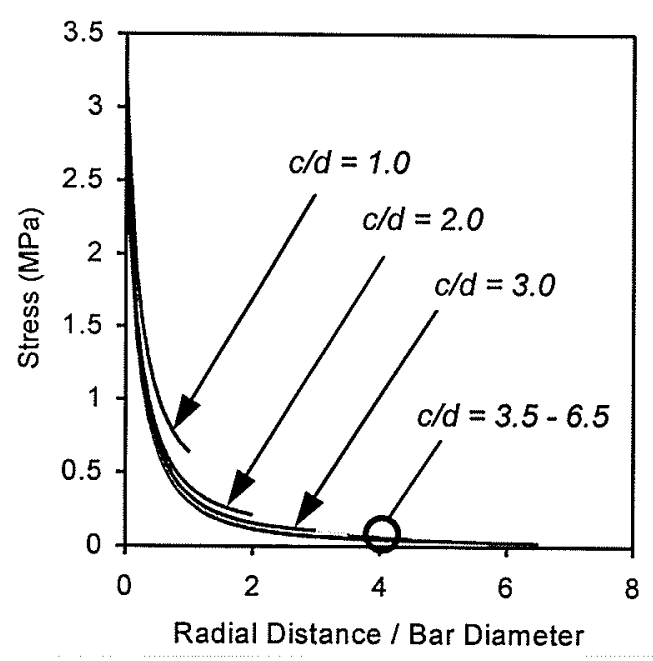

(a) $\mathrm{f}_{\mathrm{c}}=30 \mathrm{MPa}$

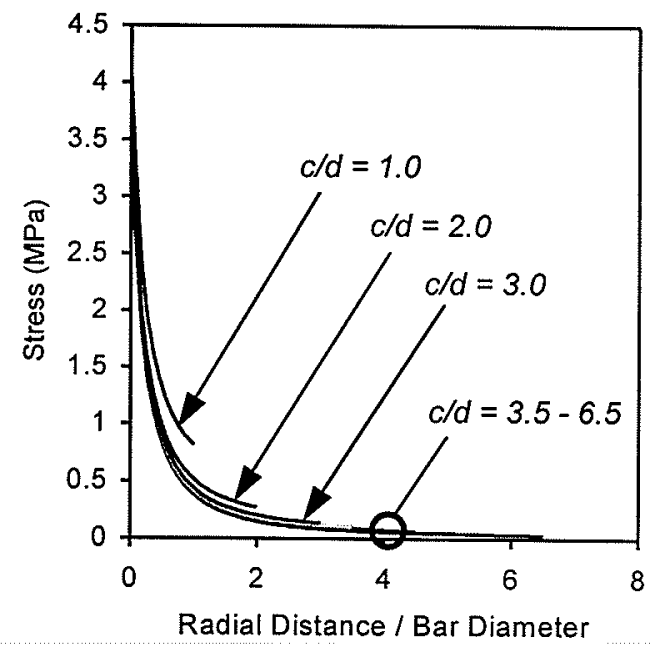

(b) $\mathrm{f}_{\mathrm{c}}=50 \mathrm{MPa}$ 


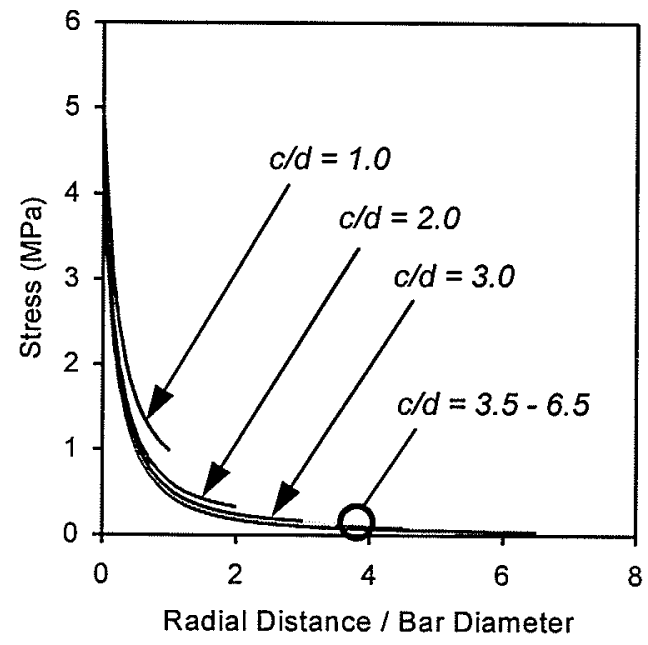

(c) $\mathrm{f}_{\mathrm{c}}^{\prime}=70 \mathrm{MPa}$

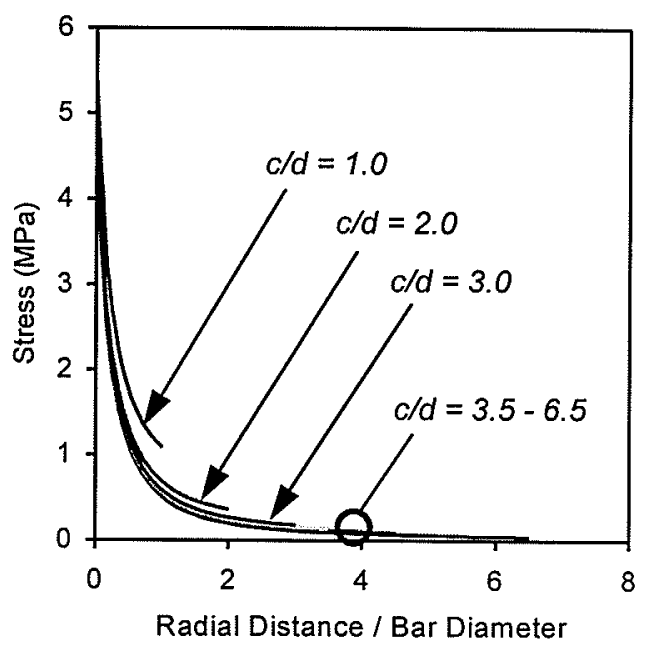

(d) $\mathrm{f}_{\mathrm{c}}=90 \mathrm{MPa}$

Figure 3.4 - Circumferential Stress Distribution Within Concrete Cover - Uncracked Analysis $\left(E_{f}^{T}=3,000 \mathrm{MPa}\right)$

$$
\frac{\partial \sigma_{\theta}}{\partial r}=-2 p b^{2} \frac{a^{2}}{b^{2}-a^{2}}\left(\frac{1}{r^{3}}\right)
$$

Figure 3.4 also demonstrates that this dissipation brings stresses to relatively small values for $\mathrm{c} / \mathrm{d}$ ratios of 3.0 and beyond, but distinctly remain larger within the cover for $\mathrm{c} / \mathrm{d}$ ratios below 3.0 . This clearly indicates that provision of small concrete covers such as those corresponding to c/d ratios of 1.0 and 2.0 is less efficient in dissipating stresses around the reinforcement. Consequently, stresses at the interface will have a tendency to remain excessive and changing the c/d ratio in this range is expected to have a noticeable effect on preventing cracks to initiate within the cover. Figure 3.5 illustrates this aspect of the linear elastic model, where circumferential stresses at the interface can be markedly reduced when increasing the concrete cover for c/d ratios below 3.0.

It can also be recognized from Figures 3.4 and 3.5 that increasing the concrete cover for $\mathrm{c} / \mathrm{d}$ ratios of 3.0 and beyond does not appreciably affect the magnitude and concentration of 
circumferential stresses near the surface of the reinforcing bar. Consequently, increasing the $\mathrm{c} / \mathrm{d}$ ratio in this range does not have a significant effect on preventing crack initiation within the cover once these stresses reach the tensile strength of concrete.

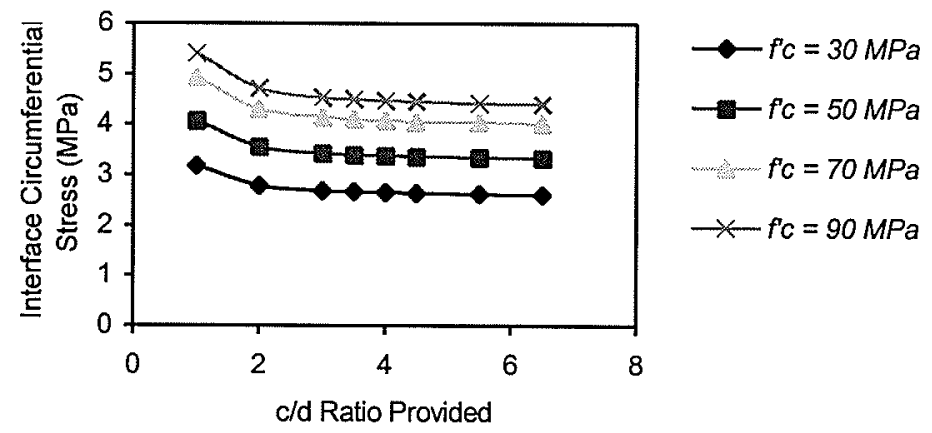

Figure 3.5 - Circumferential Stress Variation at the Interface $\left(E_{f}^{T}=3,000 \mathrm{MPa}\right)$

\subsection{Cracked Section Analysis}

Once a cracked region forms around the reinforcement, stresses within the uncracked portion of the cover taper similarly to those witnessed for the uncracked section analysis. These stresses are also found to concentrate near the interface separating the cracked region from the uncracked region. The characteristics of this distribution are illustrated in Figure 3.6 for circumferential stresses within the uncracked region of the concrete cover and material properties similar to those used in the previous section. Once more, to demonstrate the effect of increasing the concrete cover, temperature fluctuations used for the distributions presented in this figure are close to those causing complete splitting of the cover for a c/d ratio of 2.0 .

According to Figure 3.6, the distribution and concentration of circumferential stresses within the uncracked region at the edge of the cracked region remains virtually unchanged when increasing the concrete cover for $\mathrm{c} / \mathrm{d}$ ratios of 3.0 and beyond. For this reason, increasing the concrete cover beyond 3.0 does not significantly reduce the thickness of the cracked region and therefore the extent of cracking than that for $\mathrm{c} / \mathrm{d}$ ratios below 3.0. However, higher temperature fluctuations 
causing the splitting phenomenon can still be achieved by increasing the concrete cover in this range of c/d ratios, as cracks have to propagate through a larger amount of material surrounding the reinforcement before reaching the failure criteria of the linear elastic model. The thickness of the cracked region in this figure extends from the vertical axis to the tip of each curve where the maximum stress occurs.

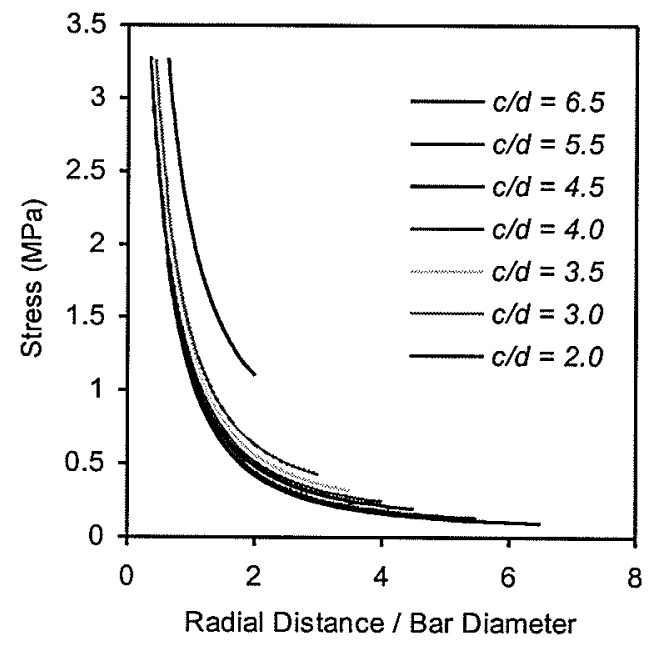

(a) $\mathrm{f}_{\mathrm{c}}^{\prime}=30 \mathrm{MPa}$

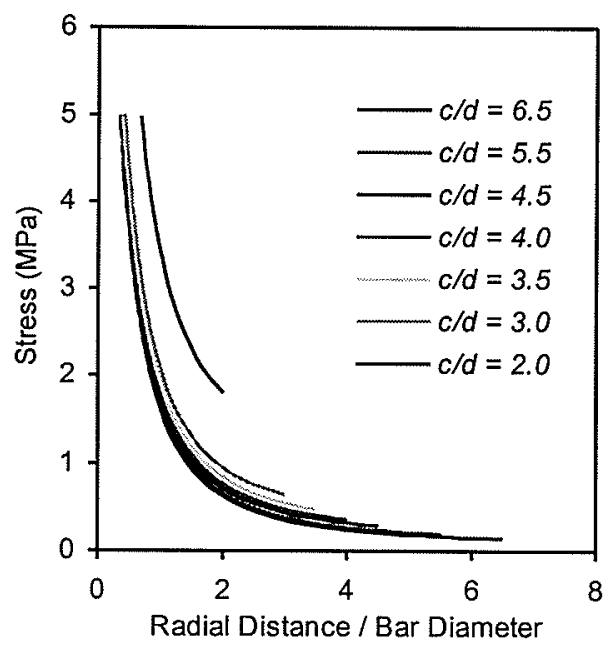

(c) $f_{c}=70 \mathrm{MPa}$

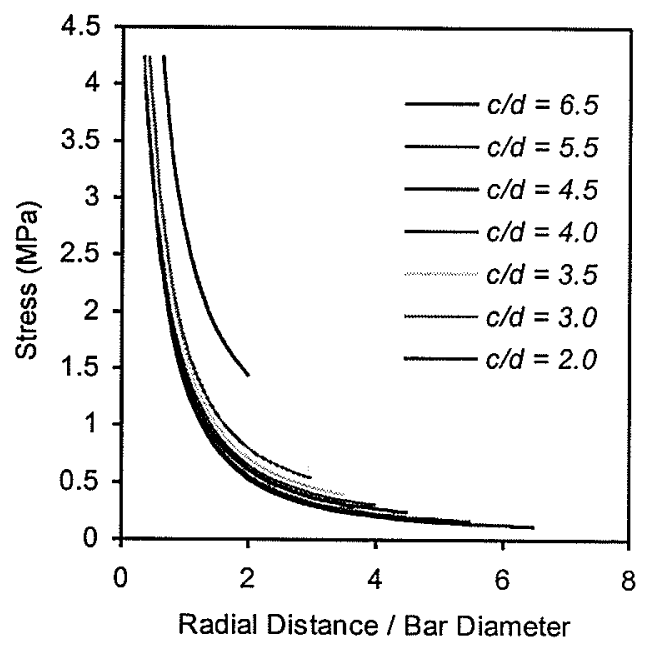

(b) $\mathrm{f}_{\mathrm{c}}=50 \mathrm{MPa}$

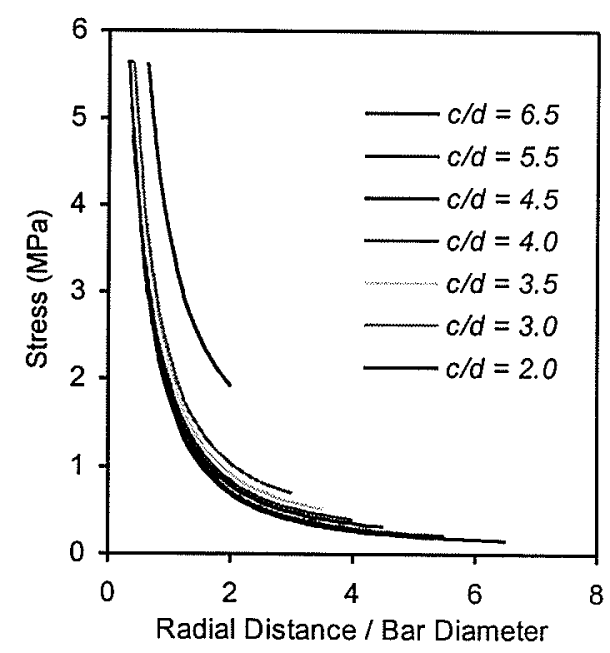

(d) $\mathrm{f}_{\mathrm{c}}=90 \mathrm{MPa}$

Figure 3.6 - Circumferential Stress Distribution Within Concrete Cover - Cracked Analysis

$$
\left(E_{f}^{T}=3,000 \mathrm{MPa}\right)
$$




\subsection{Critical Temperature Change}

The purpose of the linear elastic analysis presented in this thesis lies in producing charts that relate the maximum temperature change that can be subjected to a structural element without causing instigation of cracking or complete splitting of the concrete cover to that provided. This temperature change is referred to as the critical temperature change and is described in the sections that follow.

\subsubsection{Instigation of Cracking}

The critical temperature change causing the first appearance of cracks within the concrete cover of a structural element can be determined on the basis of expressions presented for an uncracked section analysis. In light of this, the maximum circumferential stress within the cover of an uncracked section is set equal to the tensile strength of concrete. Recalling that circumferential stresses for this type of analysis are maximum at the surface of the reinforcement where the radial distance $r$ is equal to the radius of reinforcement $a$, an expression relating the tensile strength to the radial pressure $p$ exerted by the reinforcement can be obtained.

$f_{t}=\beta p$

EQ.3.32

Further noticing that the radial pressure in the previous equation can be solely written in terms of the temperature fluctuation for a known c/d ratio and set of material properties, an expression defining the critical temperature change for crack initiation within the concrete cover can be derived.

$\Delta T_{\text {critical,cracking }}=\frac{f_{t}}{\beta\left(\alpha_{f}^{T}-\alpha_{c}\right)}\left(\frac{\beta+\nu_{c}}{E_{c}}+\frac{1-v_{f}^{T T}}{E_{f}^{T}}\right)$

EQ.3.33 


\subsubsection{Splitting Phenomenon}

The critical temperature change causing splitting of the concrete cover is determined on the basis of the failure criterion considered for a post-cracking analysis. The failure criterion is based on a maximum radial compression (EQ.3.29) causing the radius of the cracked region $r_{c r}$ to extend 48 percent of the concrete cylinder radius b (EQ.3.30). Noticing once more that the radial pressure $p^{\prime}$ for a post-cracking analysis (EQ.3.23) can be written as a function of the temperature fluctuation alone for a known c/d ratio and set of material properties, an expression defining the critical temperature change for complete splitting of the concrete cover can be derived.

$$
\Delta T_{\text {critical }, \text { splitting }}=0.3 \frac{f_{t} b}{a\left(\alpha_{f}^{T}-\alpha_{c}\right)}\left(\frac{\ln (0.48 b / a)+\beta^{\prime}+\nu_{c}}{E_{c}}+\frac{1-v_{f}^{T T}}{E_{f}^{T}}\right)
$$

\subsubsection{Sensitivity Analysis}

The critical temperature change is mainly affected by three parameters. These include the transverse CTE and transverse elastic modulus of the reinforcement as well as the compressive strength of the surrounding concrete. The latter affects the tensile strength and elastic modulus used for concrete in the analysis.

According to Figure 3.7, the linear elastic analysis is considerably sensitive to changes in the aforementioned parameters. For the properties considered in the analysis, the change in critical temperature for instigation of cracking and splitting of the concrete cover can reach between 90 and 100 percent. However, the percent change in critical temperature is observed to stabilize for larger values of transverse elastic modulus and CTE, which is not the case for compressive strength. Instead, increasing the compressive strength between 20 and $100 \mathrm{MPa}$ is close to linear. In light of this discussion, it becomes apparent that changing the compressive strength of 
concrete is the most efficient method in altering the potential for crack initiation or splitting of the concrete cover.

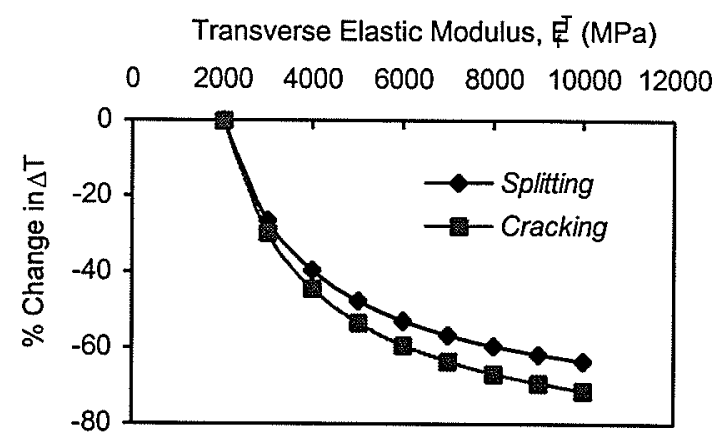

(a) Transverse Elastic Modulus of Reinforcement, $E_{f}^{\top}$

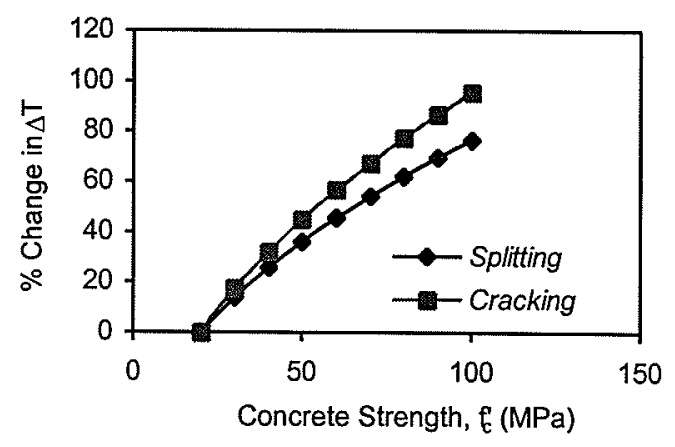

(b) Concrete Compressive Strength, $f_{c}$ Thermal Expansion Coefficient, $\alpha_{\mathrm{f}}^{\top}\left(\mu \varepsilon^{\circ} \mathrm{C}\right)$

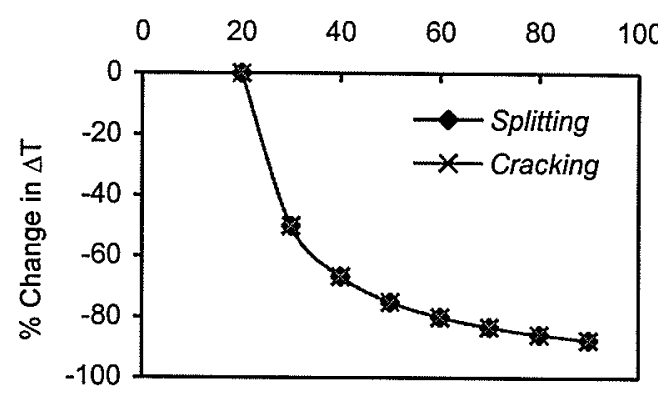

(c) Transverse Coefficient of Thermal Expansion of Reinforcement, $\alpha_{f}^{\top}$

Figure 3.7 - Sensitivity Analysis

Except in the case of the thermal expansion coefficients, it is also observed from Figure 3.7 that changing the transverse modulus and compressive strength causes a consistently higher percent change in critical temperature for the case of crack instigation than for the case of splitting. 


\subsection{Critical Temperature Charts for Placement of FRP Reinforcement}

Plotting the critical temperature change described in the previous sections against the c/d ratios considered for the analysis produces charts for determining the placement of FRP reinforcement in structural members. More specifically, the minimum concrete cover required to prevent crack initiation or complete splitting can be determined from these charts for a temperature fluctuation expected in service. The temperature fluctuation expected in service can be modified to include the effects of axial compatibility and the Hoyer effect through the use of Equation 3.21.

\subsubsection{Instigation of Cracking}

Figure 3.8 displays the critical temperature change corresponding to crack initiation (EQ.3.33) as a function of the c/d ratios considered for the analysis. As expected from the distribution of stresses, the figure illustrates that increasing the concrete cover for c/d ratios ranging from 1.0 to 3.0 has a noticeable effect on the critical temperature change. On the other hand, increasing the concrete cover for c/d ratios of 3.0 and beyond does not appreciably affect the critical temperature change that can be achieved in service and crack initiation becomes difficult to prevent in this range.

\subsubsection{Splitting Phenomenon}

Figure 3.9 displays the critical temperature change corresponding to the splitting phenomenon (EQ.3.34) as a function of the c/d ratios considered for the analysis. It is apparent for all c/d ratios that increasing the concrete cover produces a constant increase in the temperature fluctuation that can be achieved in service without occurrence of the splitting phenomenon. Accordingly, the main conclusion arising from the linear elastic analysis is the fact that increasing 
the concrete cover in structural elements is more efficient in preventing the occurrence of the splitting phenomenon than it is for preventing the occurrence of cracks.

\subsubsection{Procedure for Determining Minimum Concrete Cover}

Based on a set of known material properties, the minimum concrete cover required to prevent instigation of cracking and complete splitting from occurring in a structural element can be determined from Figures 3.8 and 3.9, respectively. Each line in the figure corresponds to a distinct FRP reinforcement transverse CTE value measured in $\mu \varepsilon /{ }^{\circ} \mathrm{C}$. The procedure is described below and divided into four individual steps.

(1) Determine the equivalent temperature change for the analysis, considering, where applicable:

- Transverse Thermal Incompatibility

- Longitudinal Thermal Incompatibility

- The Hoyer Effect

(2) Obtain the c/d ratio corresponding to the expected temperature change from the charts. Figure 3.8 can be used to determine the $\mathrm{c} / \mathrm{d}$ ratio corresponding to initiation of cracking and Figure 3.9 can be used to determine the c/d ratio corresponding to complete splitting of the concrete cover.

(3) Calculate the concrete cover required to prevent cracking or the splitting phenomenon on the basis of the bar diameter used in the design.

(4) Provide a concrete cover greater than or equal to that calculated for the expected temperature change. 


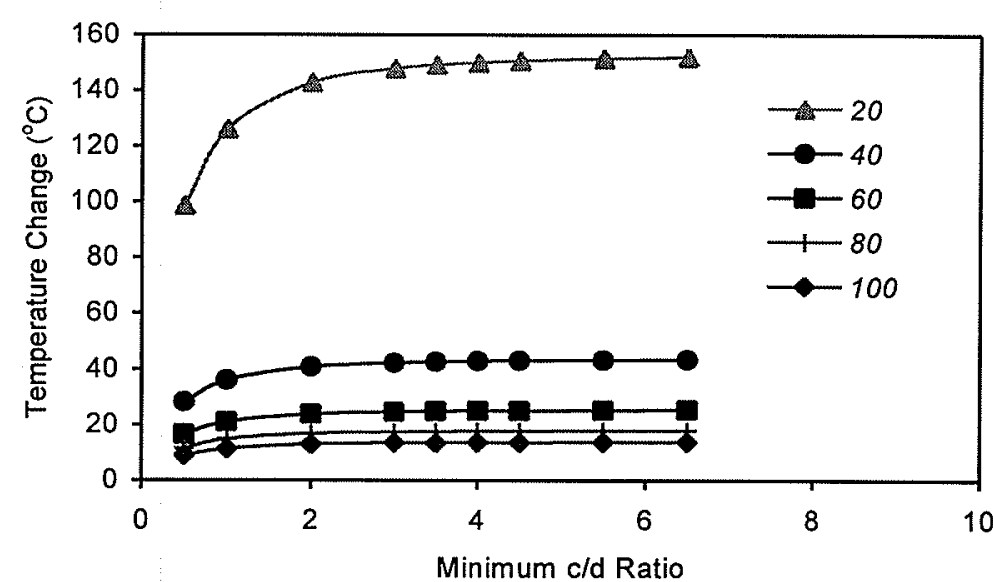

(a) $f_{c}=30 \mathrm{MPa}, E_{f}^{\top}=2,000 \mathrm{MPa}$

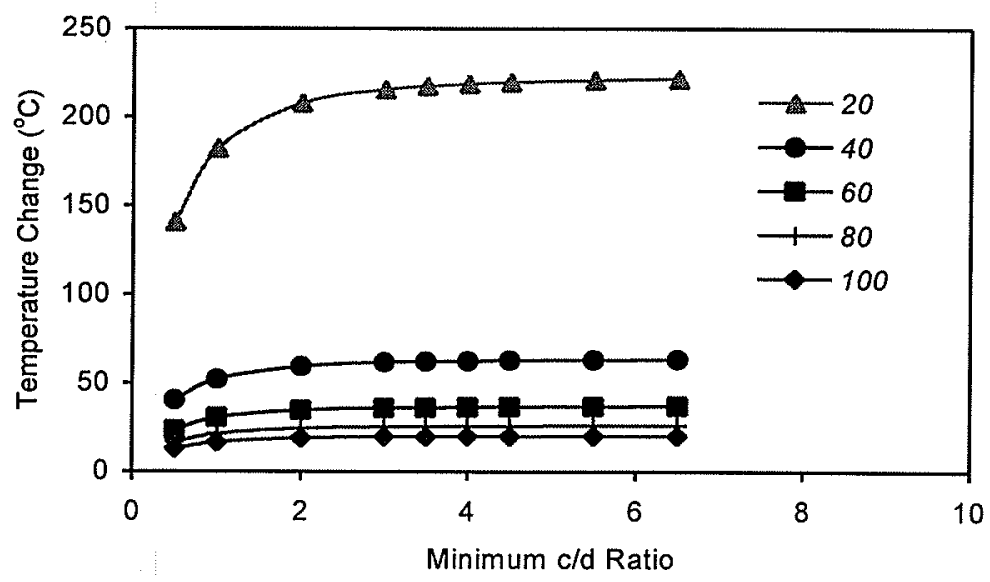

(c) $\mathrm{f}_{\mathrm{c}}^{\prime}=70 \mathrm{MPa}, \mathrm{E}_{\mathrm{f}}^{\top}=2,000 \mathrm{MPa}$

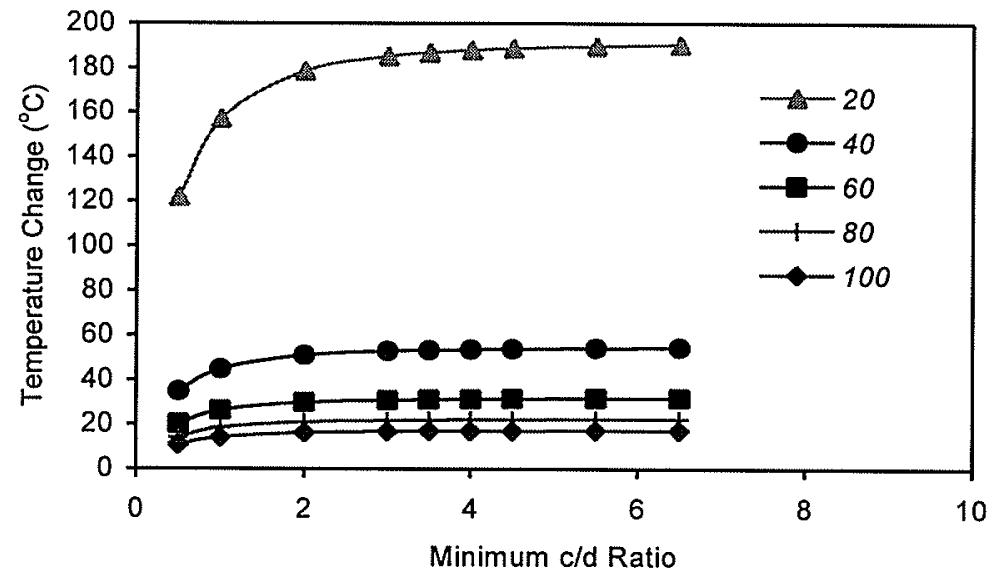

(b) $\mathrm{f}_{\mathrm{c}}^{\prime}=50 \mathrm{MPa}, \mathrm{E}_{\mathrm{f}}^{\top}=2,000 \mathrm{MPa}$

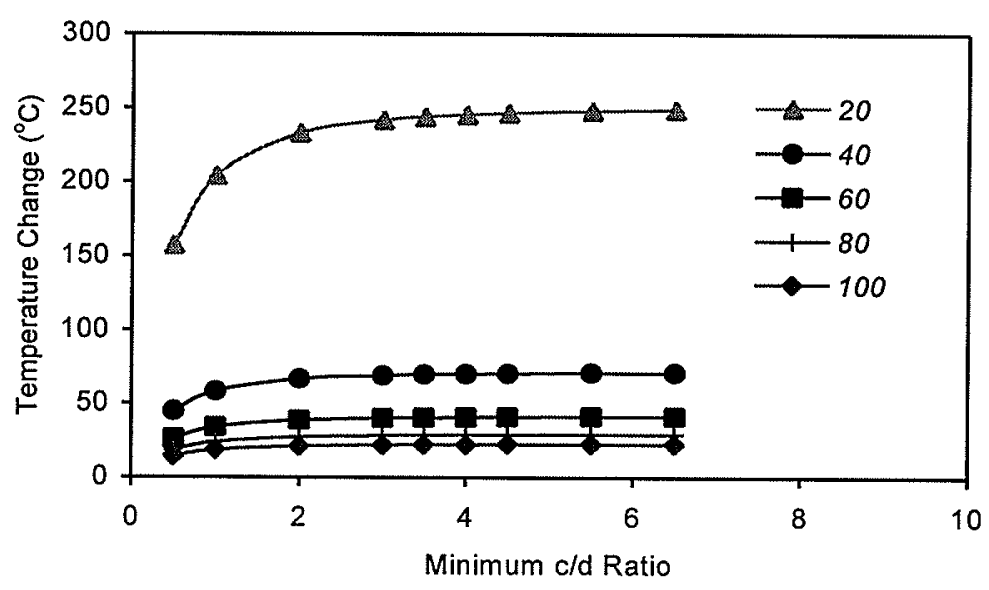

(d) $f_{c}^{\prime}=90 \mathrm{MPa}, E_{f}^{\top}=2,000 \mathrm{MPa}$ 


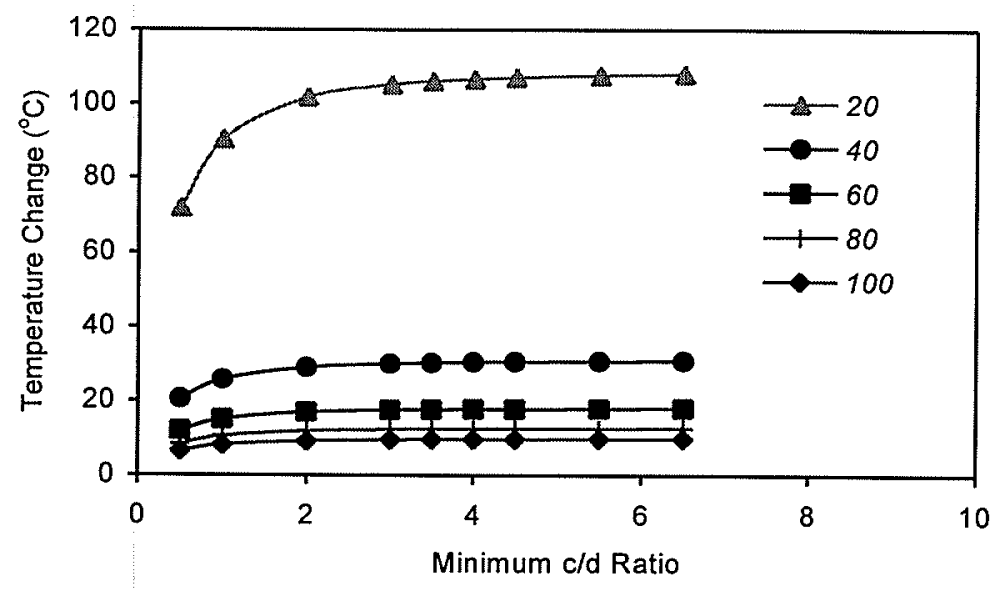

(e) $f_{c}^{\prime}=30 \mathrm{MPa}, E_{f}^{\top}=3,000 \mathrm{MPa}$

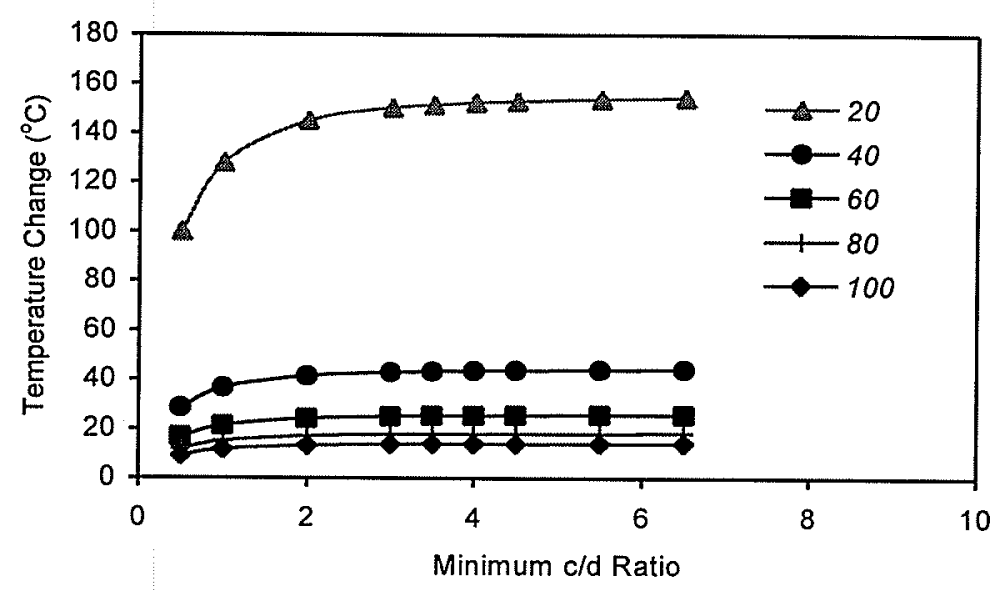

(g) $f_{c}^{\prime}=70 \mathrm{MPa}, E_{f}^{\top}=3,000 \mathrm{MPa}$

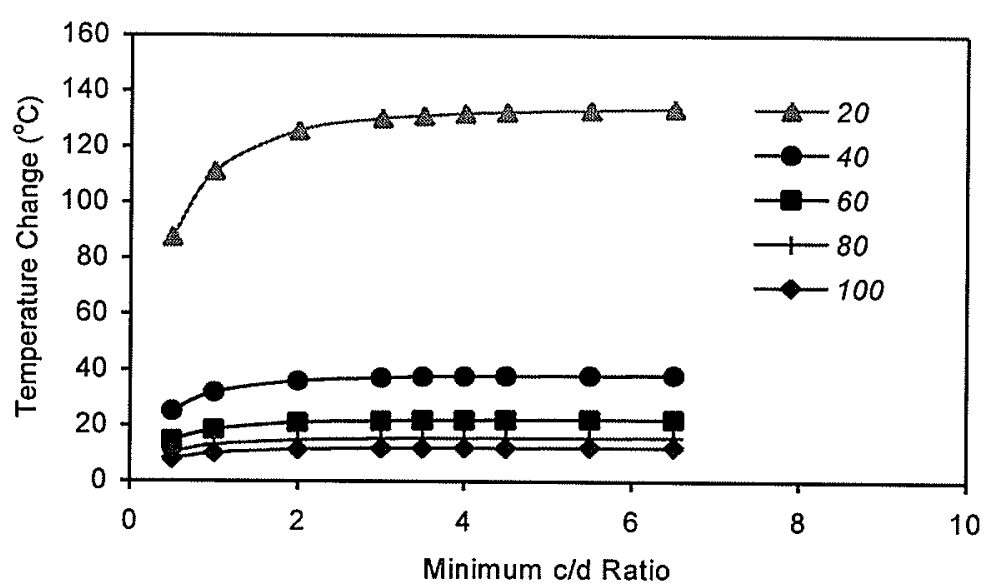

(f) $f_{c}=50 \mathrm{MPa}, E_{f}^{\top}=3,000 \mathrm{MPa}$

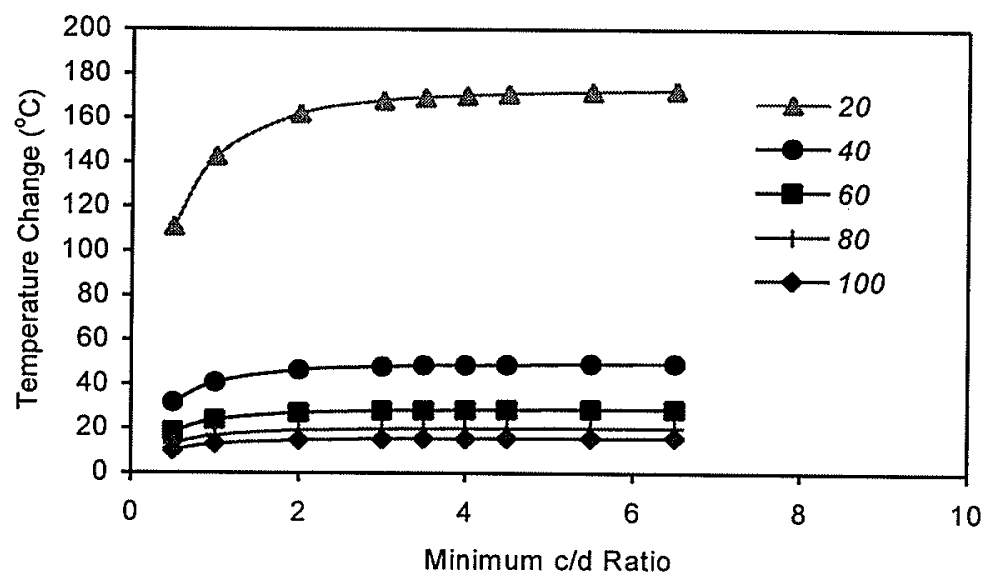

(h) $f_{c}^{\prime}=90 \mathrm{MPa}, E_{f}^{\top}=3,000 \mathrm{MPa}$ 

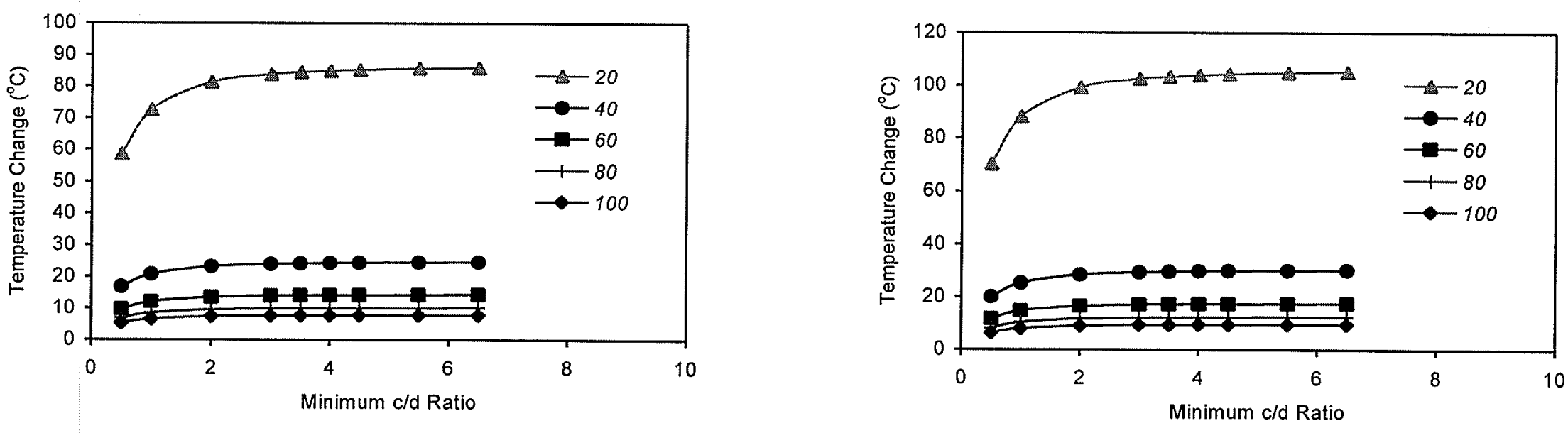

(i) $f_{c}^{\prime}=30 \mathrm{MPa}, E_{f}^{\top}=4,000 \mathrm{MPa}$
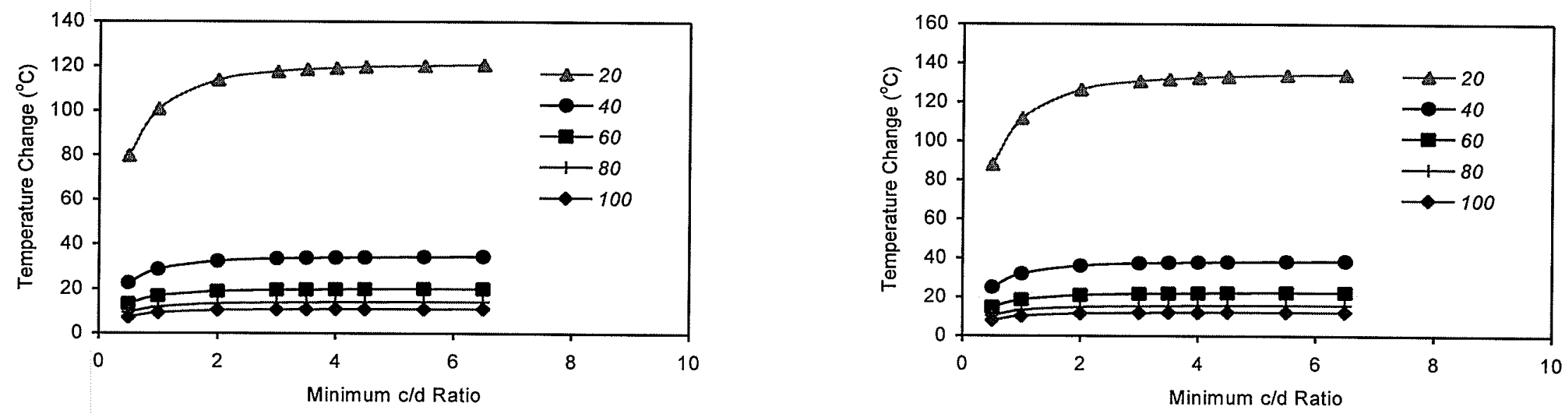

(k) $\mathrm{f}_{\mathrm{c}}^{\prime}=70 \mathrm{MPa}, \mathrm{E}_{\mathrm{f}}^{\top}=4,000 \mathrm{MPa}$

(I) $\mathrm{f}_{\mathrm{c}}^{\prime}=90 \mathrm{MPa}, \mathrm{E}_{\mathrm{f}}^{\top}=4,000 \mathrm{MPa}$ 


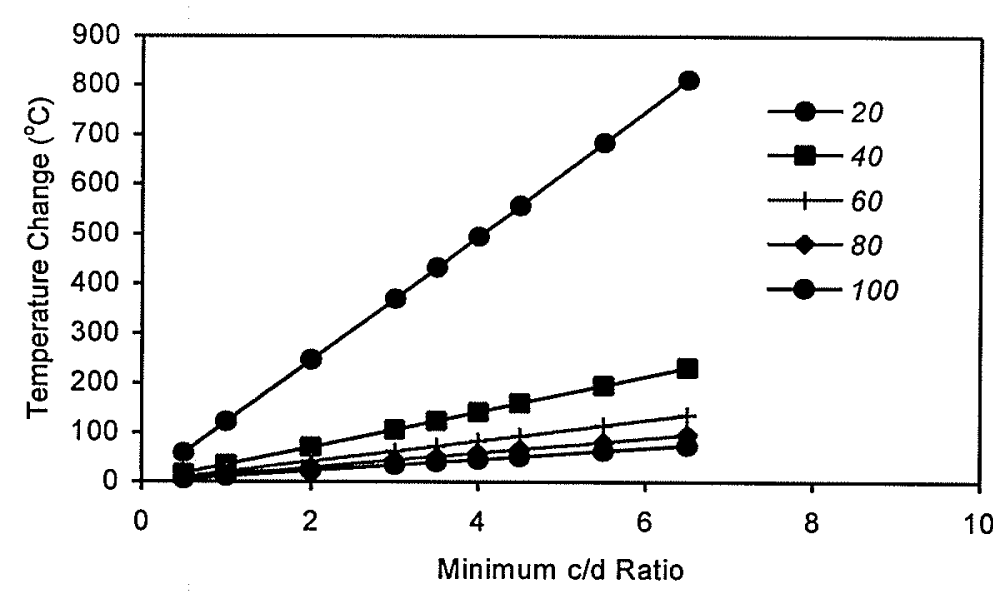

(a) $f_{c}^{\prime}=30 \mathrm{MPa}, E_{f}^{\top}=2,000 \mathrm{MPa}$

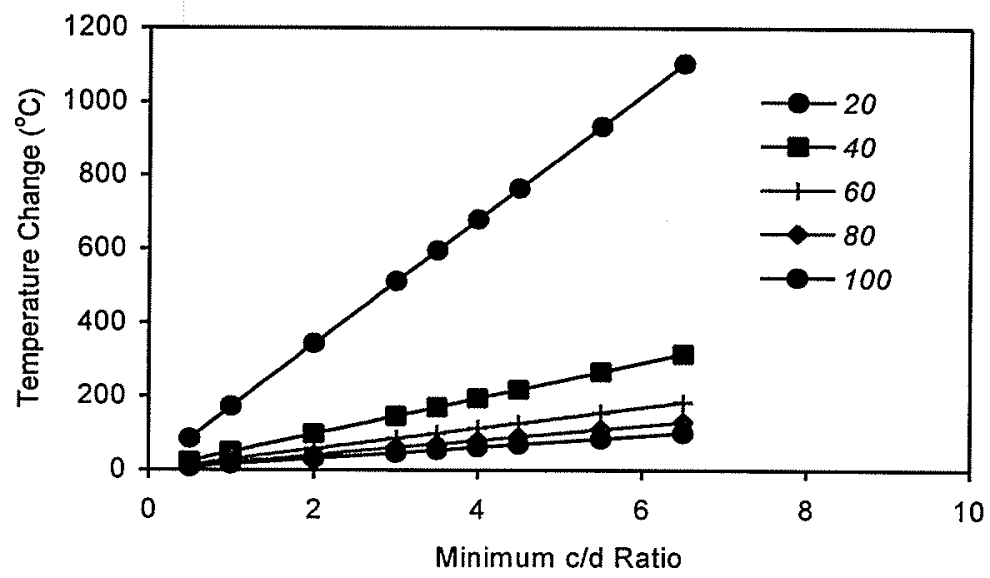

(c) $f_{c}^{\prime}=70 \mathrm{MPa}, E_{f}^{\top}=2,000 \mathrm{MPa}$

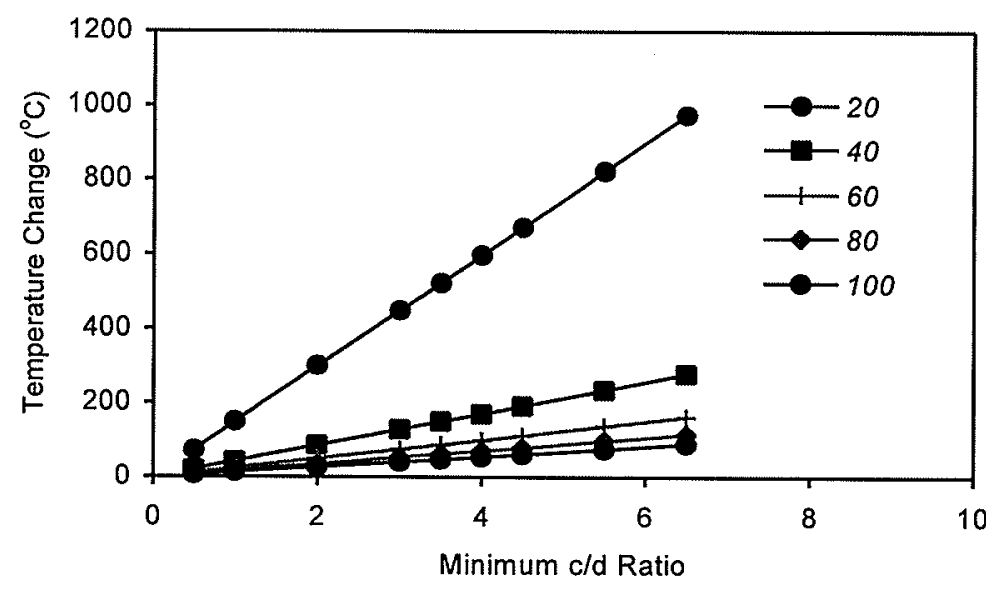

(b) $f_{c}^{\prime}=50 \mathrm{MPa}, E_{f}^{\top}=2,000 \mathrm{MPa}$

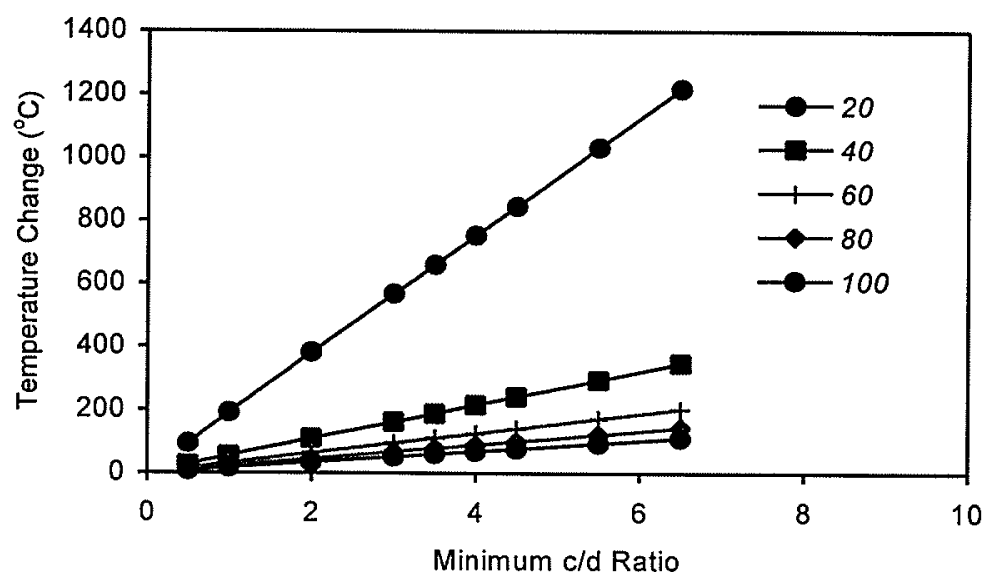

(d) $\mathrm{f}_{\mathrm{c}}^{\prime}=90 \mathrm{MPa}, \mathrm{E}_{\mathrm{f}}^{\top}=2,000 \mathrm{MPa}$ 


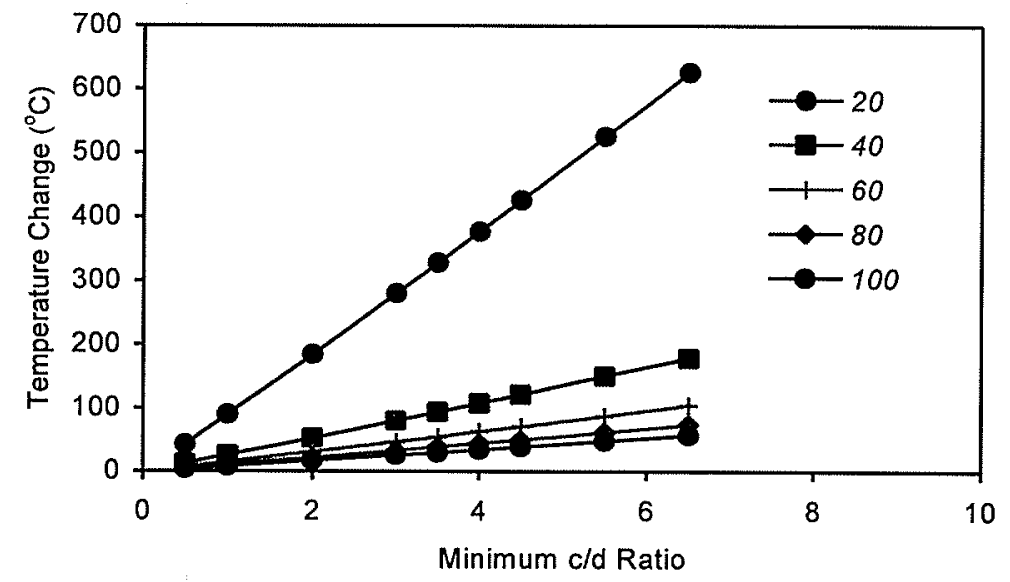

(e) $f_{c}^{\prime}=30 \mathrm{MPa}, E_{f}^{\top}=3,000 \mathrm{MPa}$

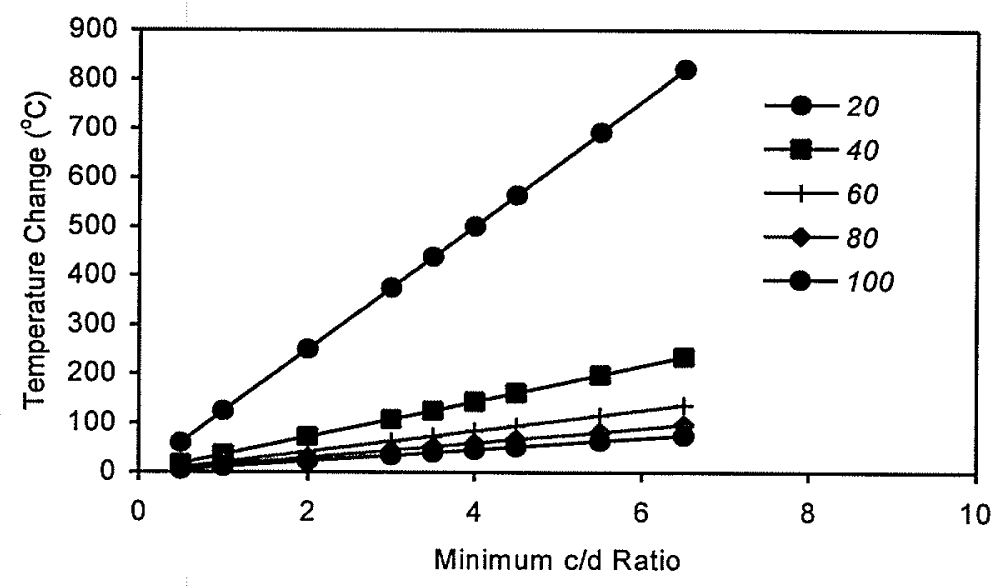

(g) $f_{c}^{\prime}=70 \mathrm{MPa}_{1} E_{f}^{\top}=3,000 \mathrm{MPa}$

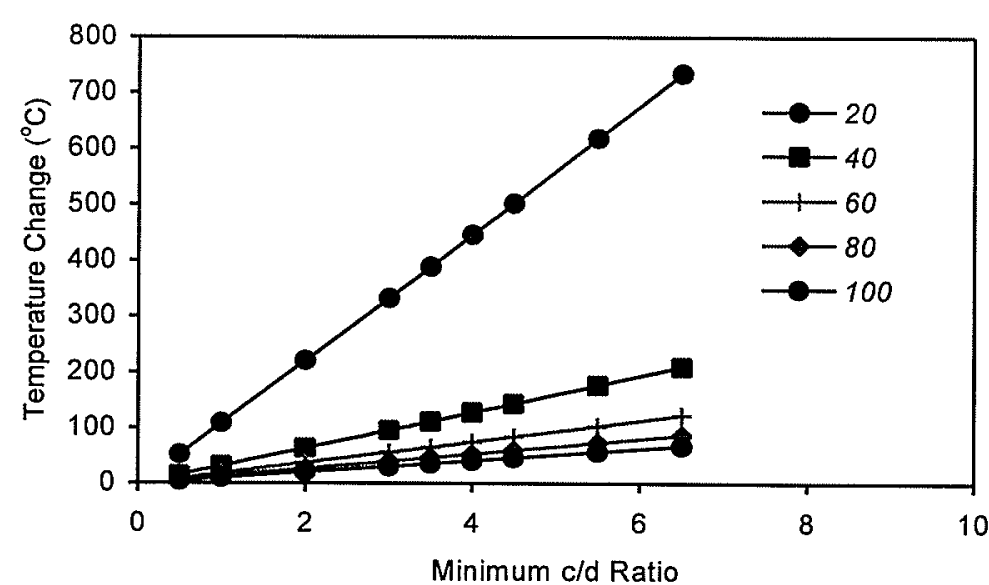

(f) $f_{c}=50 \mathrm{MPa}, E_{f}^{\top}=3,000 \mathrm{MPa}$

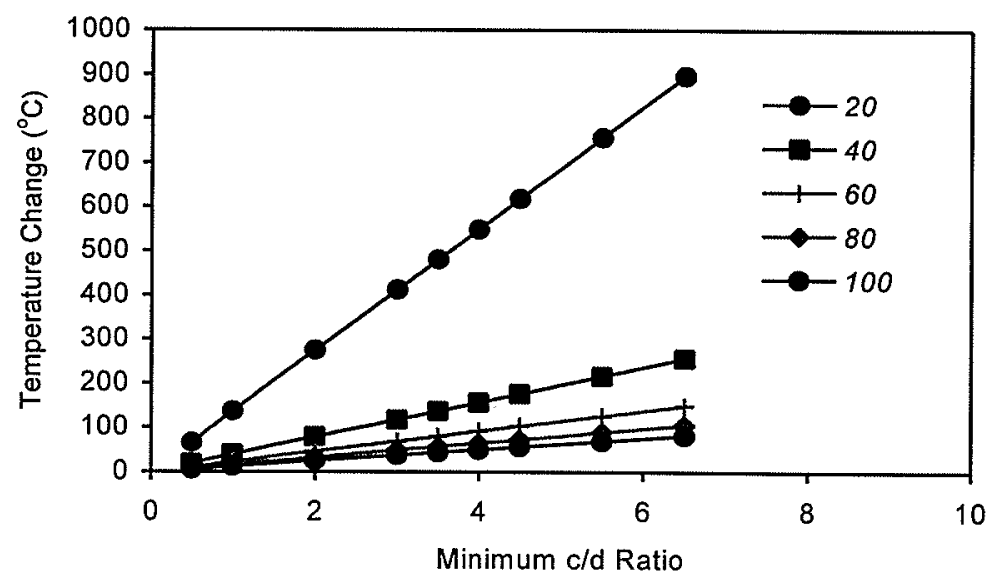

(h) $\mathrm{f}_{\mathrm{c}}=90 \mathrm{MPa}, \mathrm{E}_{\mathrm{f}}^{\top}=3,000 \mathrm{MPa}$

Figure 3.9-Minimum c/d Ratio - Splitting Phenomenon 

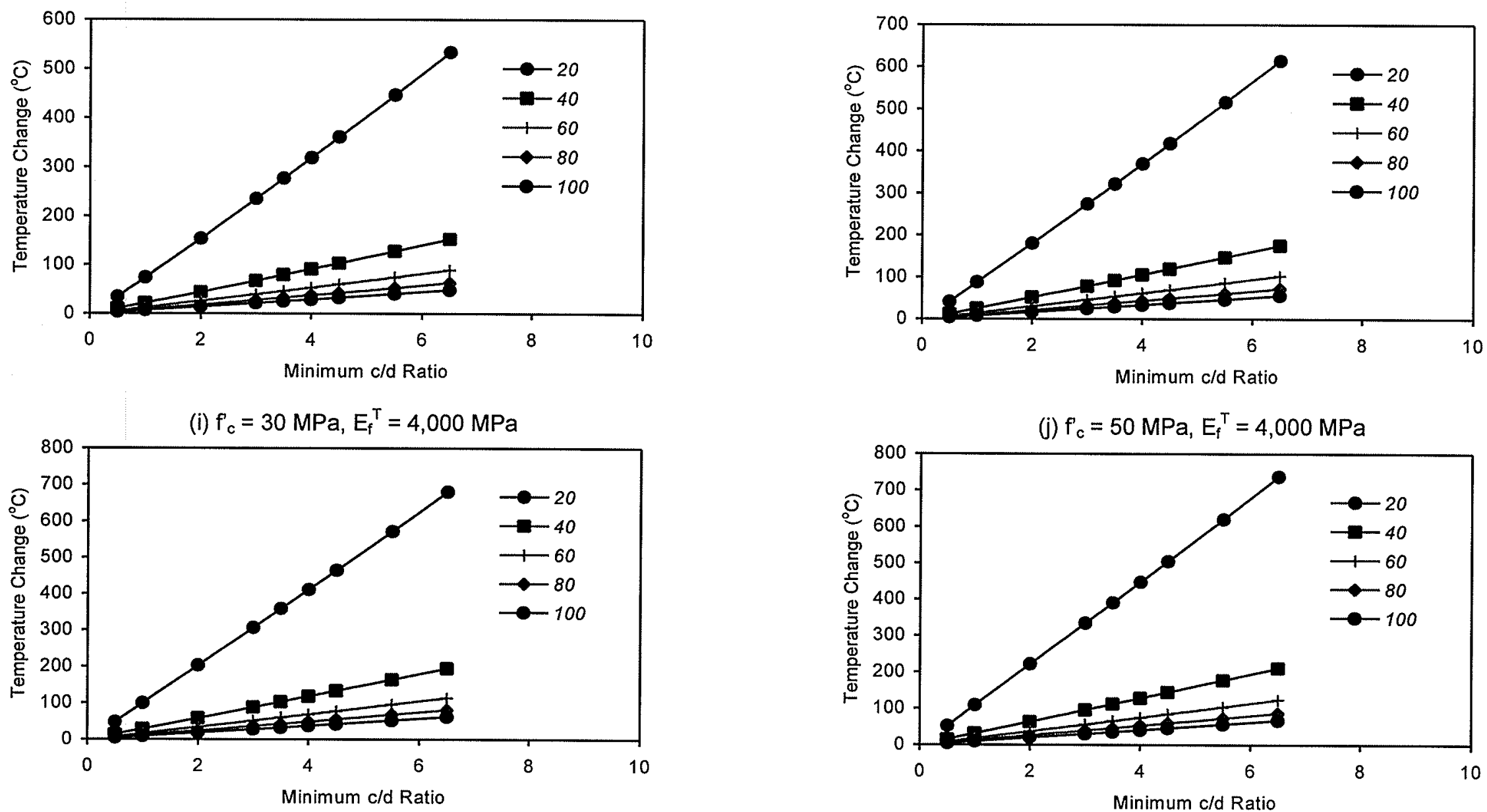

(k) $\mathrm{f}_{\mathrm{c}}=70 \mathrm{MPa}, \mathrm{E}_{\mathrm{f}}^{\top}=4,000 \mathrm{MPa}$

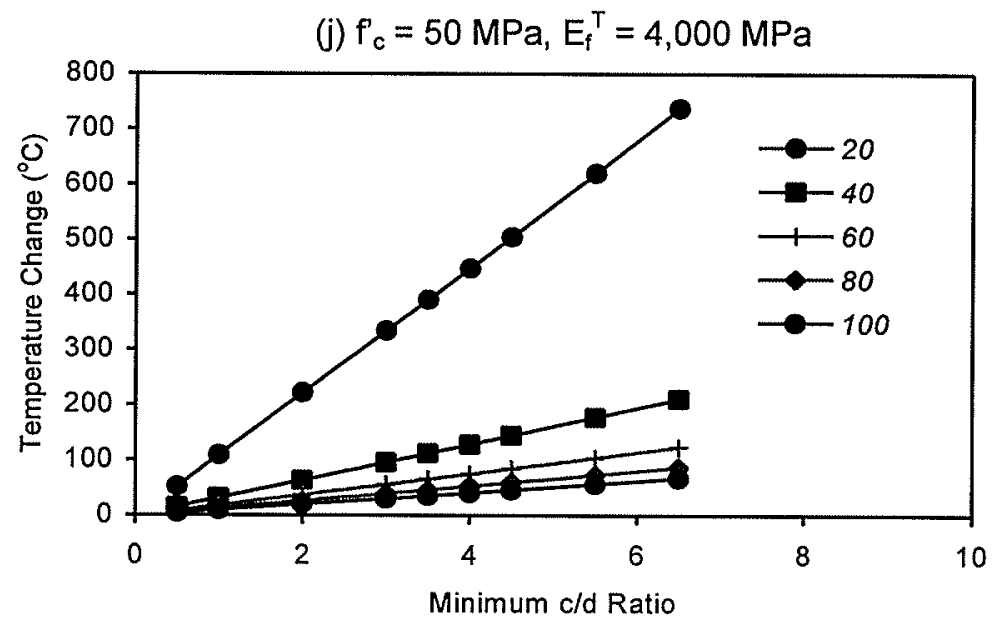

(I) $\mathrm{f}_{\mathrm{c}}^{\prime}=90 \mathrm{MPa}, \mathrm{E}_{\mathrm{f}}^{\top}=4,000 \mathrm{MPa}$

Figure 3.9 - Minimum c/d Ratio - Splitting Phenomenon 


\section{Chapter 4}

\section{Finite Element Analysis of Thermally Induced Stresses}

\subsection{Assumptions}

Expressions describing thermal stresses in the linear elastic model are limited by the assumption of linear elastic material behaviour. It is well known that compressive stresses within the cover can cause concrete material to show non-linear material behaviour under thermal stresses. In light of this and in reference to conclusions drawn by Aiello (1999), a finite element analysis was carried out using software called ADINA to account for non-linear material behaviour and study the development of thermally induced stresses within the concrete cover of FRP reinforced concrete elements. Yet, assumptions related to FRP material properties, bar spacing as well as crack control and shear reinforcement remain unchanged from that stated and used for the linear elastic analysis.

\subsection{Finite Element Model Representation}

Another limitation concealed by the linear elastic representation of thermally induced stresses is the geometry of the zone influenced by thermal expansion of the reinforcing bar. Due to axisymmetric assumptions involved in the derivation of expressions defining the linear elastic model, the geometry is limited to that of a concrete cylinder surrounding the reinforcement. Consequently, the finite element model was elaborated to account for prismatic geometries most likely found in structural components. The model was elaborated to consider the splitting 
phenomenon caused by FRP reinforcing bars placed in a concrete beam with equivalent side and bottom covers. Assuming these covers are sufficiently larger than the spacing separating the reinforcing bars, cracks are ensured to develop and propagate along the shortest distance to the external surface of the component. The crack pattern is shown in Figure 4.1(a). Analyzing the section affected by the splitting phenomenon and applying boundary conditions on the basis of symmetry leads to the idealization illustrated in Figure 4.1(b). The boundary conditions release shear strains and restrain normal strains along the covers of the model.

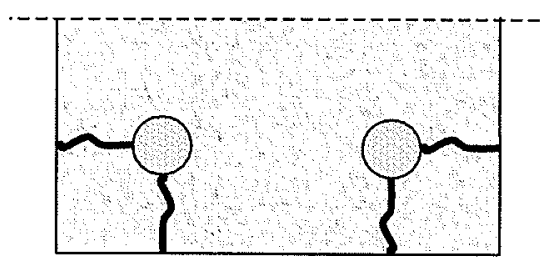

(a) Crack Pattern in Reinforced Concrete Beam

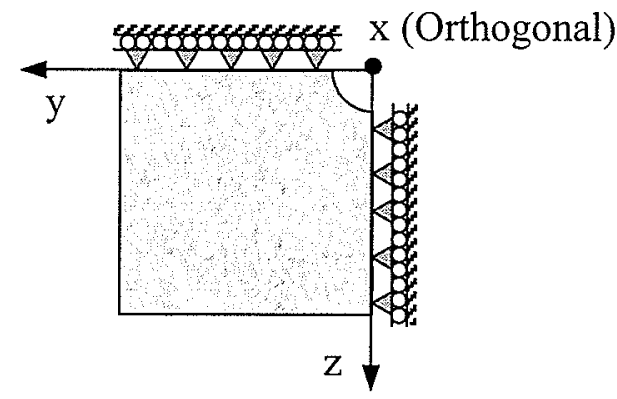

(b) Idealized Model

Figure 4.1 - Model Representation

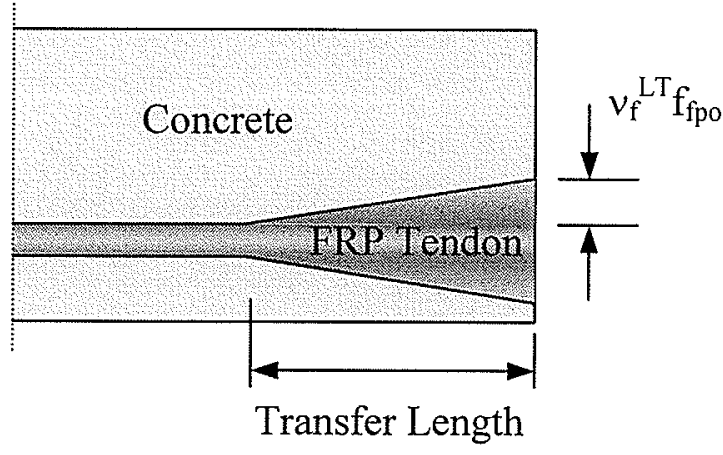

(a) Hoyer Effect

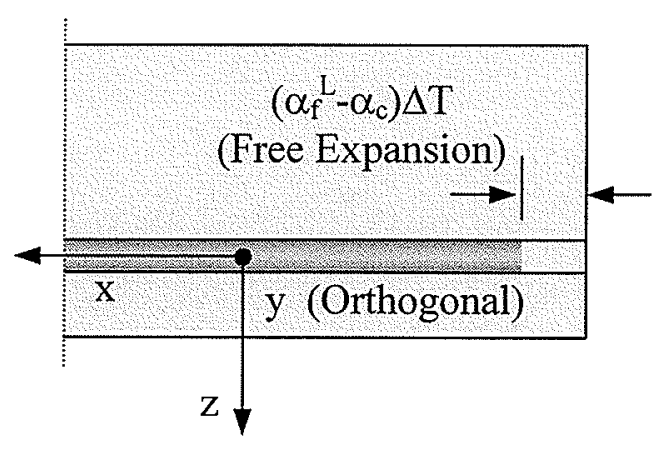

(b) Longitudinal CTE Disparity

Figure 4.2 - Sources of Longitudinal Stresses (x - Axis)

The finite element model was analyzed on the basis of two-dimensional solid elements with plane stress conditions. Plane stress conditions apply when normal and shear stresses in the third direction $\left(\sigma_{x}, \tau_{y x}, \tau_{x y}\right)$ are inexistent or noticeably minute. As illustrated in Figure 4.2, there are two sources of stress in the third orientation. The first arises from longitudinal CTE disparity and the 
requirement of axial compatibility while the bond is preserved between the materials. As observed in the linear elastic analysis, stresses arising from this source are typically small and are not expected to affect the assumption for plane stress. The second source arises from the transverse expansion of a tendon when the prestressing force is released. Due to Hoyer's effect, the maximum transverse expansion of the tendon upon release of prestress will occur at the edge of the pretensioned member where all of the prestress is released. As a result, the longitudinal stresses at this location are inexistent and the assumption of plane stress remains valid.

\subsection{Concrete Material Model}

One of the distinct advantages of the non-linear analysis software is the inclusion of a concrete model. The model is based on a uniaxial stress-strain relationship similar to the one shown in Figure 4.3. The relationship is generalized to take multiaxial stress conditions into account. The basic characteristics of this model include tensile failure at a maximum, relatively small principal tensile stress and compression crushing failure at high compression. As illustrated in Figure 4.3, the compression crushing and tension failures are governed by failure envelopes that include strain-softening effects in the tensile and compressive regions.

As cracks initiate under the presence of tensile stresses, linear strain-softening relationships are assumed and the material will lose strength through a softening mechanism. This introduces mesh sensitivity into the results and will also lead to non-converging finite element predictions, as mesh refinements will lead to narrower elements and therefore narrower crack bands. To obtain solution convergence with mesh refinement, ADINA allows the user to specify the fracture toughness of the material, $G_{f}$. This property is regarded as a material fracture parameter and represents the energy absorbed per unit area of crack. With this approach, the concrete's behaviour is characterized by a stress-displacement response similar to that shown in Figure 4.4 as opposed to a stress-strain response. The fracture toughness is defined as the area under the entire softening stress-displacement curve. 


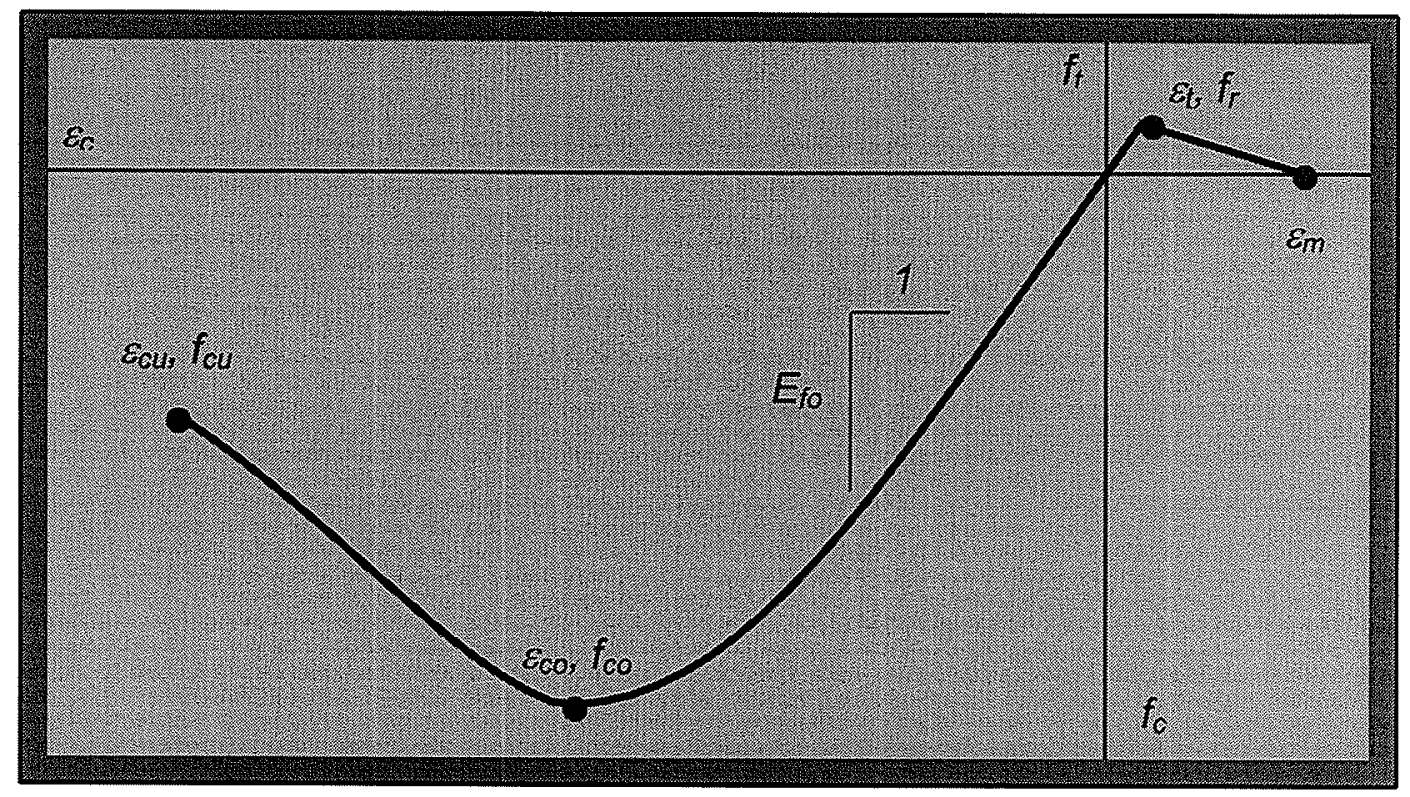

Figure 4.3 - Uniaxial Stress-Strain Curve for Concrete

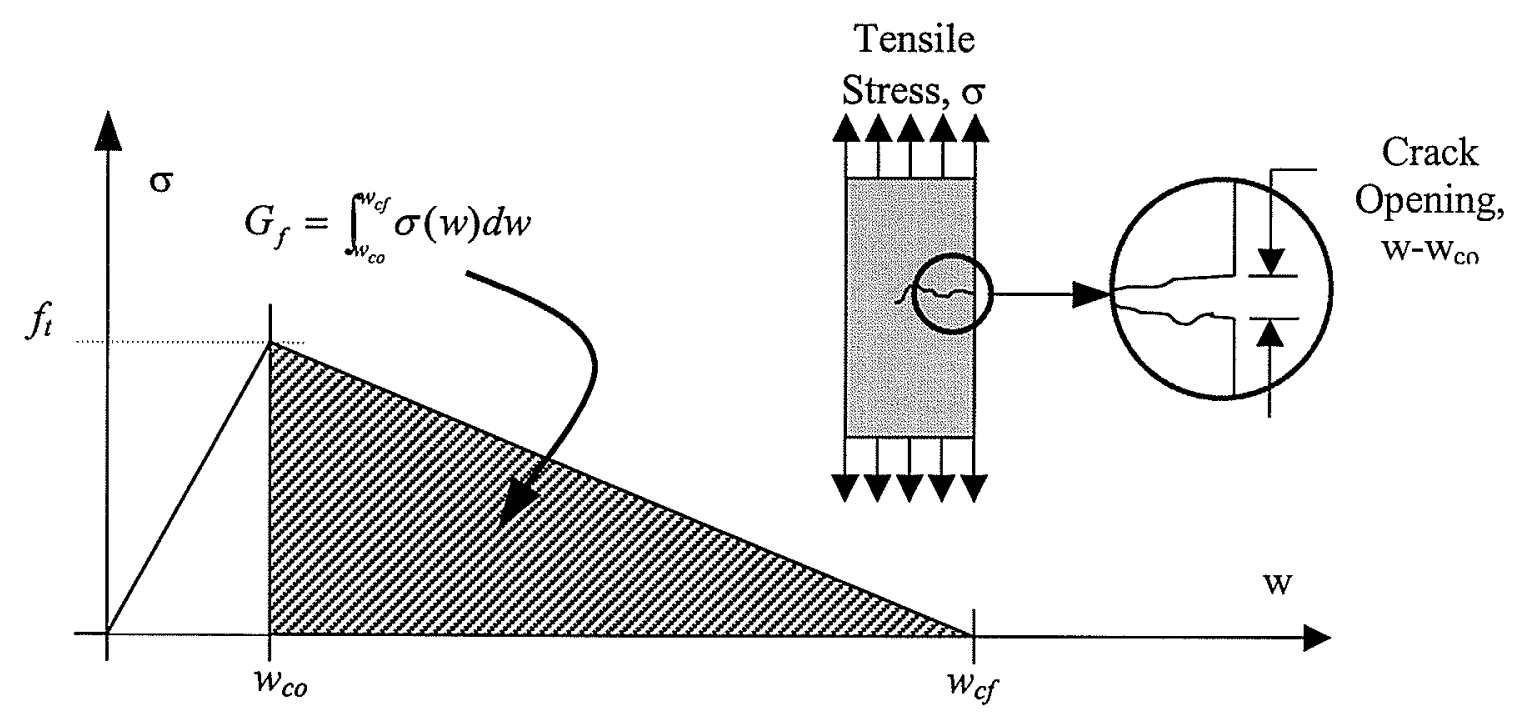

Figure 4.4 - Complete Tensile Stress-Displacement Curve

Tensile failures resulting from initiation of cracking occur when tensile stresses in a principal stress direction exceed the tensile failure stress $f_{t}$. It is then assumed that a plane of failure develops perpendicular to the corresponding principal stress direction. This material failure causes a reduction in the normal and shear stiffness across the plane of failure. ADINA takes 
into account this effect by introducing normal stiffness and shear reduction factors that need to be specified by the user. The normal stiffness reduction factor $\eta_{s}$ is a small value, typically $10^{-4}$, and is not set exactly to zero for avoiding the possibility of a singular stiffness matrix. Conversely, the shear reduction factor $\eta_{n}$ was set at a value of 0.5 typically used for non-linear finite element analyses involving concrete material (Mao et al., 1997). However, this value is overwritten whenever the fracture toughness is provided.

\subsection{FRP Reinforcement Model}

As emphasized in Chapter 2 of this thesis, a total of three independent Poisson ratios exist for FRP reinforcement. However, for a state of plane stress as that selected for the finite element analysis, only one of these ratios is required. If we consider a state of plane stress for which $\sigma_{z}=$ $\tau_{y z}=\tau_{z y}=0$, stress-strain relationships only require Poisson's ratio relating strains in orientations that are transverse to each other in the zy-plane, namely $v_{f}^{\pi}$. Similarly, the elastic modulus of the reinforcement required for the plane stress analysis of the finite element model is that relating stress and strain in the zy-plane, namely $E_{f}^{T}$. Along with the transverse CTE, these material properties are essential for modelling and including FRP reinforcement in the finite element analysis.

As opposed to that for concrete, ADINA does not provide a built in material model for FRP reinforcement. Based on the previous discussion, the reinforcement was defined as a thermo isotropic material in the plane of interest with Poisson's ratio, transverse elastic modulus and transverse CTE specified for the range of temperatures considered in the analysis. The user can readily change these properties and parameters, which is one of the main advantages of the finite element analysis software. This is also the case for the concrete model described in the previous section. 


\subsection{Finite Elements}

The concrete material was modelled using 8-node quadrilateral isoparametric finite elements. The formulation for these elements is displacement based and allows the consideration of nonrectangular shapes and curved sides as required at the surface of reinforcing bars. Figure 4.5 also shows that FRP reinforcing bars were modelled with 6-node triangular isoparametric finite elements formulated using coordinates and methods similar to those for quadrilateral isoparametric finite elements.

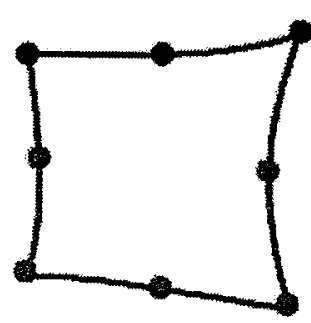

(a) Rectangular (Concrete)

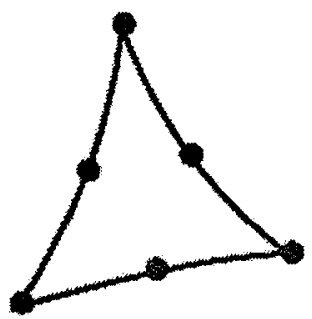

(b) Triangular (FRP)

Figure 4.5 - Isoparametric Finite Elements

Two element groups were created to identify the properties and parameters of each material. Different mesh colours were used to differentiate materials in the finite element model. Lines and surfaces defining the model as well as the mesh implemented in ADINA are illustrated in Figure 4.6 and confirm the type of element used for each material.

\subsection{Solution Process}

Since non-linear material behavior is incorporated into the analysis, caution and good judgment must be used in choosing the solution process. ADINA provides three distinct incremental solution methods. These include the modified Newton Raphson method, the BFGS (BroydenFletcher-Goldfarb-Shanno) method and the Full Newton Raphson method. These are all iterative procedures with the most expensive one being the Full Newton Raphson method. As illustrated 
in Figure 4.7 for an arbitrary function $f(a)$, the efficiency level of the method is mainly governed by the recalculation of stiffness at each step. However, with the use of a line search, the Full Newton Raphson method can achieve convergence more rapidly than any of the other methods. For that reason, the Full Newton Raphson method with line search was used in the analysis presented herein. The solution process is usually recommended for use when excessive nonlinearities are encountered (Zienkiewicz and Taylor, 1999).

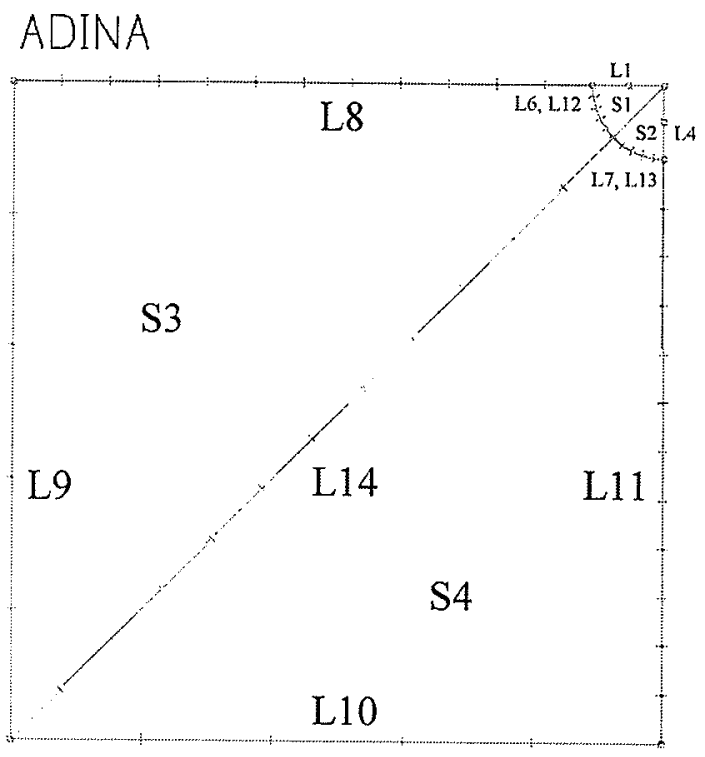

(a) Line (L) and Surface (S) Depiction
ADINA

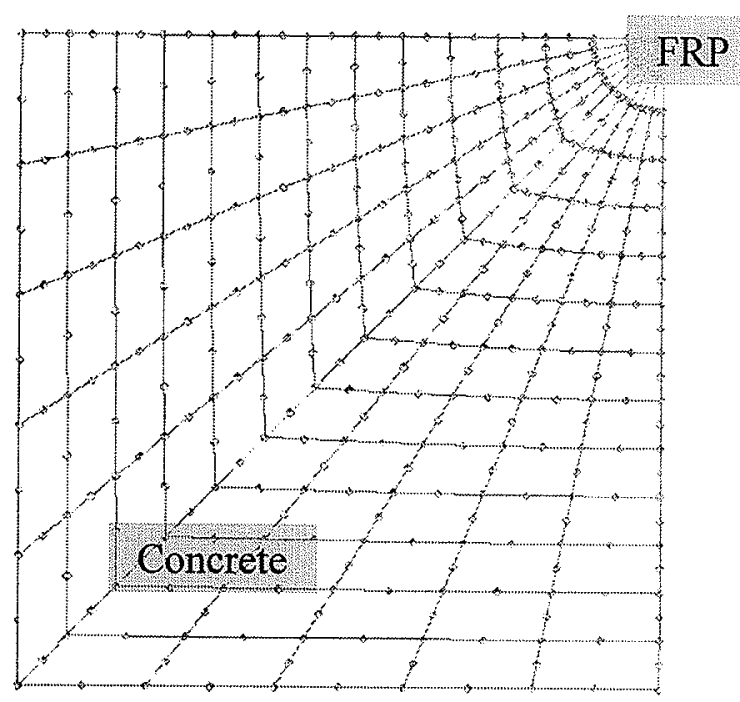

(b) Finite Element Mesh

Figure 4.6 - Finite Element Model (ADINA)

The software also provides an Automatic-Time-Stepping (ATS) method. The method is launched when time steps specified by the user are too large to meet convergence criteria. When convergence fails, the method subdivides existing time steps until a solution is reached. The method is applicable with any of the incremental solution methods.

\subsection{Stress Distribution Within Cover}

One of the main objectives of the finite element model was to study the development of stress in FRP reinforced concrete elements subjected to thermal gradients. Accordingly, the finite element 
analysis was carried out with two programs of the ADINA software. The first program is called ADINA-T and was used to subject desired temperature levels to the model. Temperature was applied on the external boundaries of the model as illustrated in Figure 4.8(a). The second program is called ADINA and was used to study displacements and stresses arising from imposed temperature levels. Figure $4.8(\mathrm{~b})$ shows the model implemented in ADINA and the boundary conditions adopted. Variables $U_{2}$ and $U_{3}$ represent displacements along the $y$ and $z$ axes, respectively. Check marks indicate released degrees of freedom in the figure. It should also be noted from the figure that the choice of element and mesh density were kept the same in both programs of the software during the analysis. This allowed temperature levels to be mapped from ADINA-T to the same integration points in ADINA where thermally induced stresses were calculated. Results from the analysis were used to establish critical temperature charts as those obtained from the linear elastic analysis.

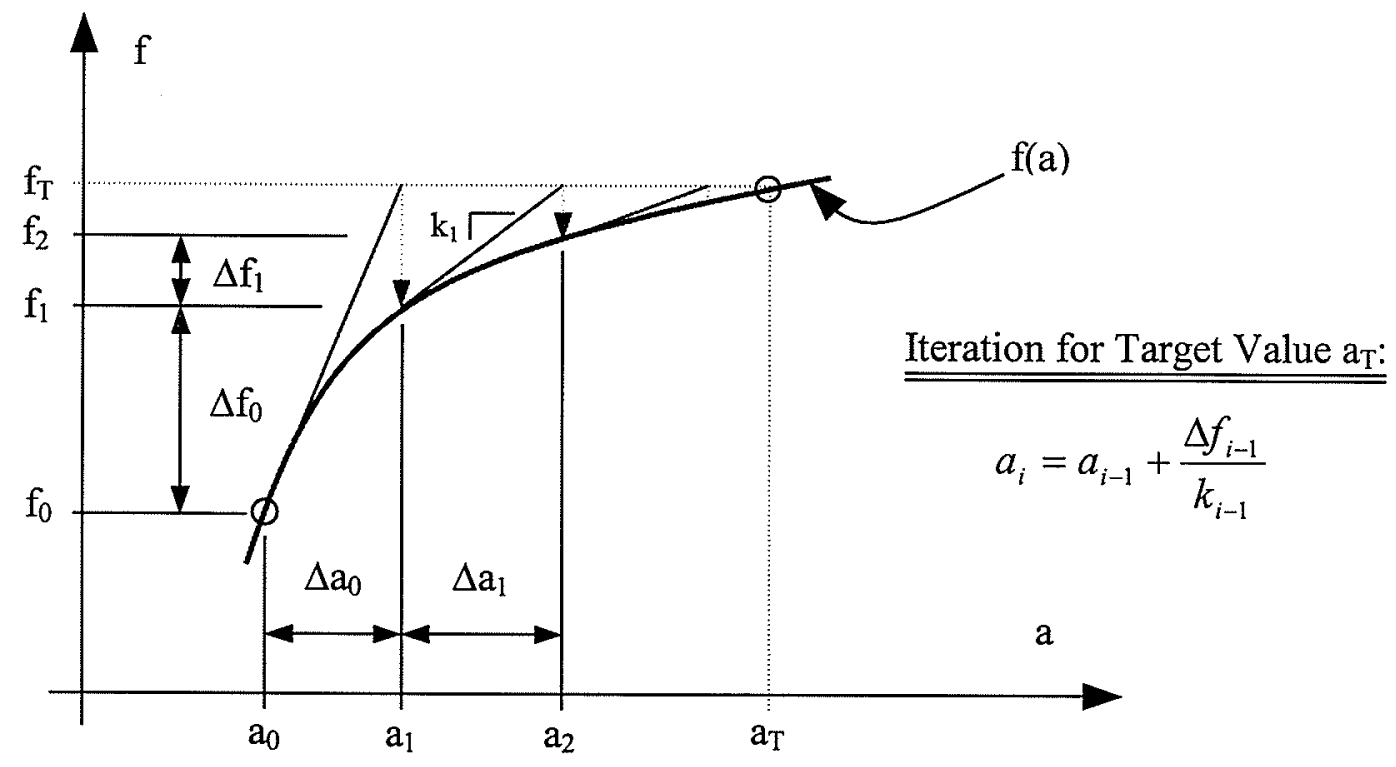

Figure 4.7 - Full Newton Raphson Iterative Procedure

As observed from the linear elastic analysis, regions surrounding the reinforcing bar were the most affected by differential swelling of the materials. Since both analyses are concerned with damage arising from cracking of concrete adjacent to swelling FRP reinforcement, tensile 
stresses were studied and acquired from the finite element analysis. Noteworthy is the fact that ADINA interprets positive stresses as tensile and negative stresses as compressive. Positive, maximum principal stresses will therefore represent maximum tensile stresses within the model. Maximum principal stresses (SIGMA-P1) within the concrete cover of the model are illustrated in Figure 4.9 for geometrical and material properties indicated. The stresses have units of MPa and are noticeably higher in the vicinity of the reinforcement. As expected, the stresses appear to dissipate with distance from the reinforcement.

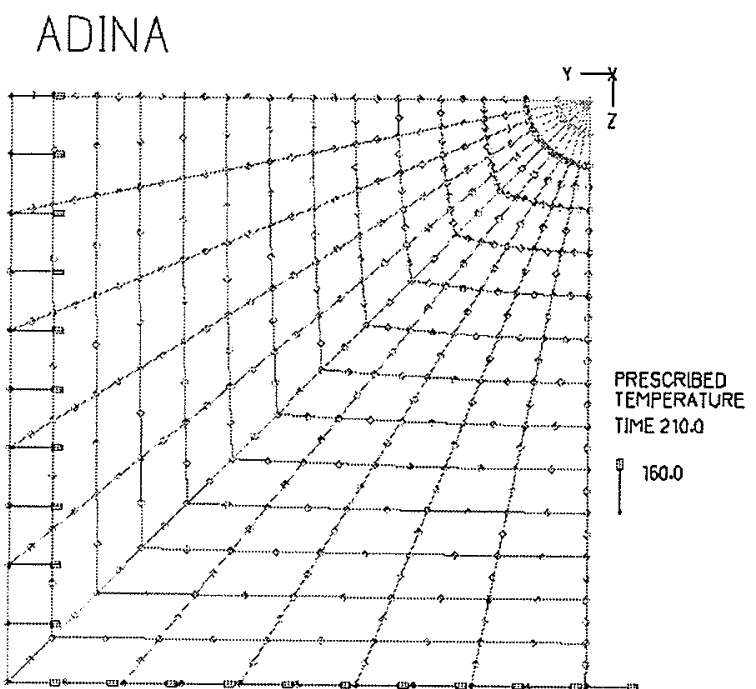

(a) Application of Temperature (ADINA-T)

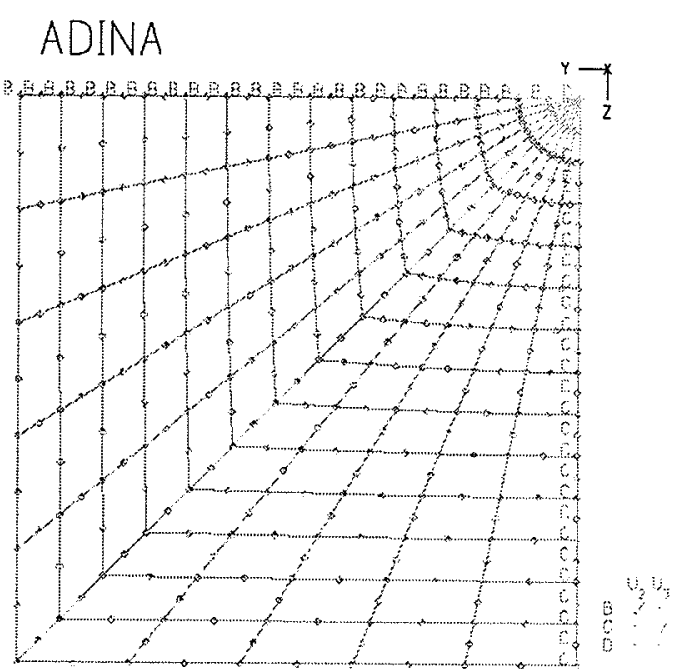

(b) Thermal Stress Analysis (ADINA)

Figure 4.8 - Finite Element Analysis Programs and Boundary Conditions

Since the concrete cover varies between the horizontal and vertical sides of the model, it is also expected for the stress distribution to alter around the bar. Stresses should appear higher along lines defining the shortest concrete cover and lower along lines defining the largest concrete cover. The stress distribution along the diagonal portion of the model should therefore demonstrate ability of the model to distribute stresses more evenly within the cover. Consequently, stresses at the interface between the materials should be smaller than those revealed for the side and bottom covers when considering results along diagonal line L14 in Figure 4.6. 

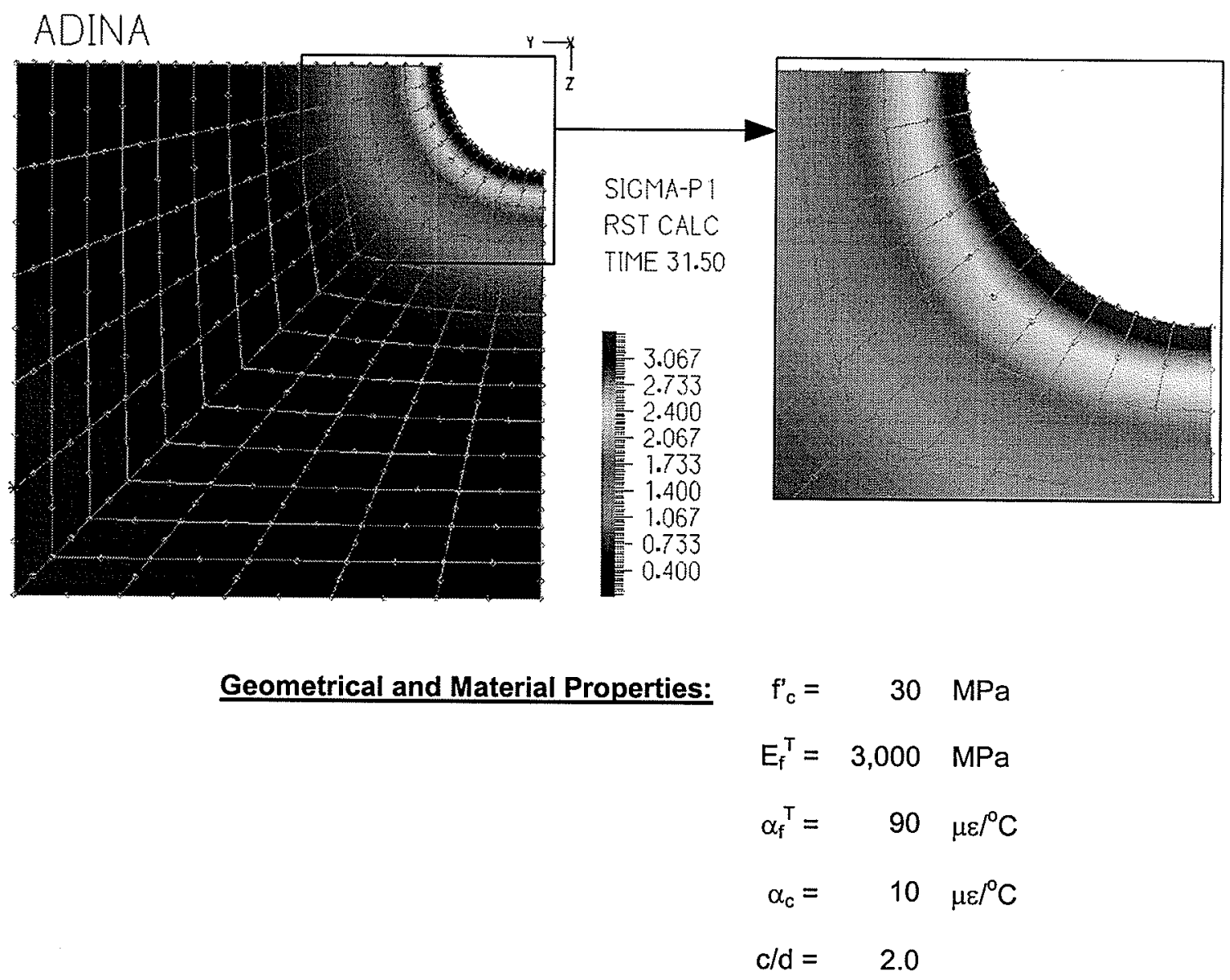

Figure 4.9 - Principal Stress Distribution Within Cover (130 elements, 417 nodes)

In accordance with the previous discussion and Figure 4.6, the stress distribution was studied along the shortest concrete cover defined by horizontal line L8 and vertical line L11 of the finite element model. It was also obtained along diagonal line L14 forming a 45-degree angle with the horizontal. As shown in Figure 4.10 for c/d ratios of 4.0 and 7.0 , stress distributions obtained from the finite element model were slightly different than those obtained from the linear elastic analysis. The solid lines represent first principal stresses obtained from the finite element model. On the other hand, markers describe the stress distribution obtained either from the linear elastic analysis in Figure 4.10 or along the diagonal cover of the finite element model in Figure 4.11 for c/d ratios of 4.0 and 7.0. Results correspond to several thermal gradients before initiation of 
cracking and demonstrate the progression of stress within the concrete cover as temperature increases. Geometrical and material properties are listed in the figures.

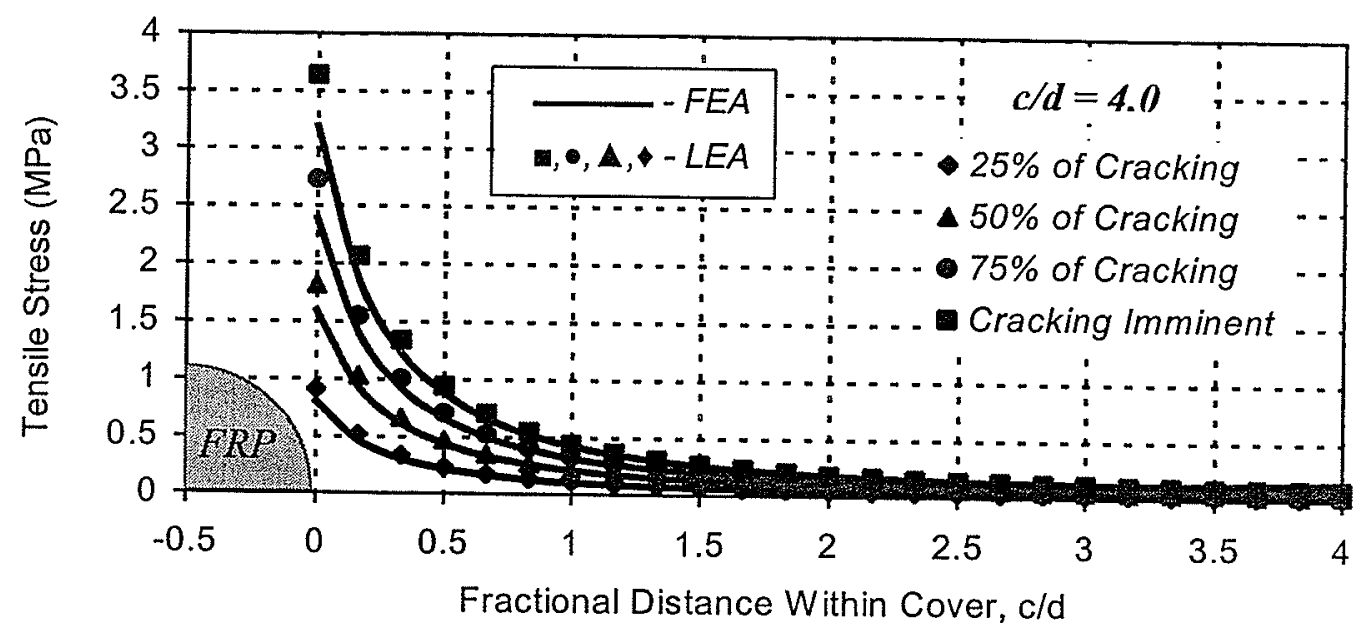

(a) Concrete Cover to Bar Diameter Ratio $=4.0$

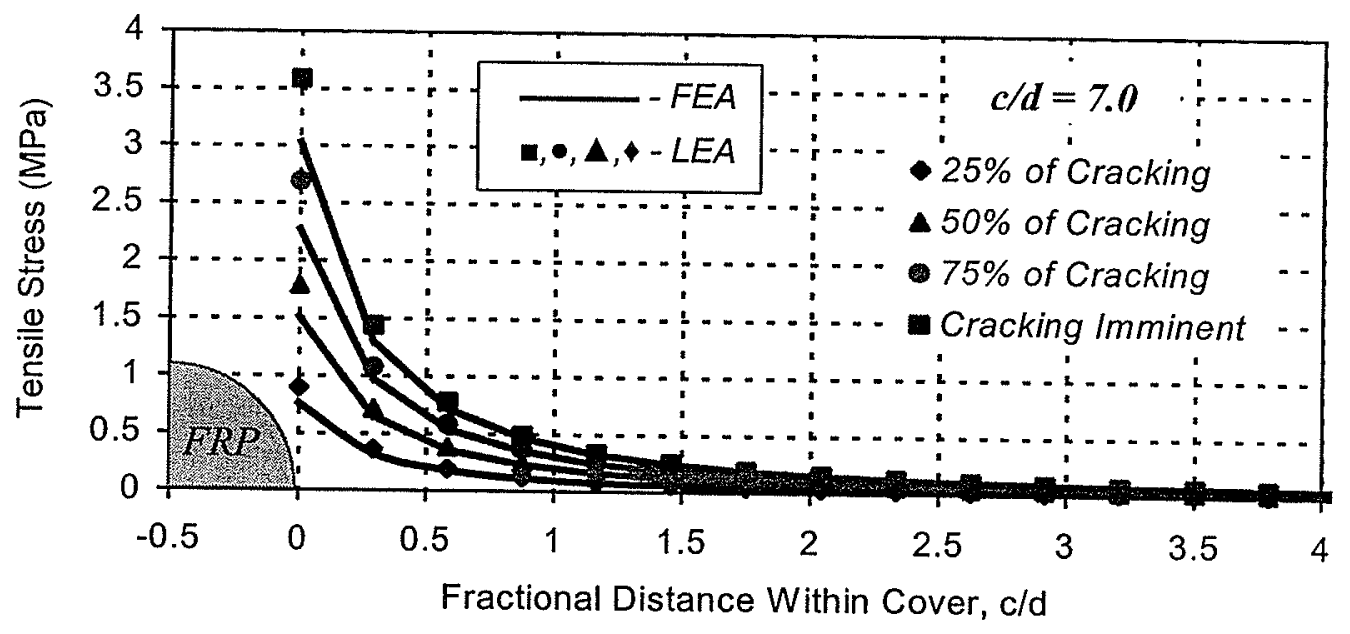

(b) Concrete Cover to Bar Diameter Ratio $=7.0$

Figure 4.10 - First Principal Stress Distribution Along Shortest Cover

$$
\left(f_{c}^{\prime}=30 \mathrm{MPa}, \mathrm{E}_{\mathrm{f}}^{\top}=3,000 \mathrm{MPa}, \alpha_{\mathrm{f}}^{\top}=90 \mu \varepsilon /{ }^{\circ} \mathrm{C}\right)
$$

According to Figure 4.10, stresses at the interface are slightly lower and tend to dissipate at a lower rate than that obtained from the linear elastic analysis. As will be discussed and illustrated later, higher temperatures to initiate cracking within the concrete cover of the finite element model will result. It is also notable from Figure 4.11 that, although slightly inferior, stresses along the 
diagonal cover (L14) are particularly close to those along the horizontal (L8) and vertical (L11) covers.

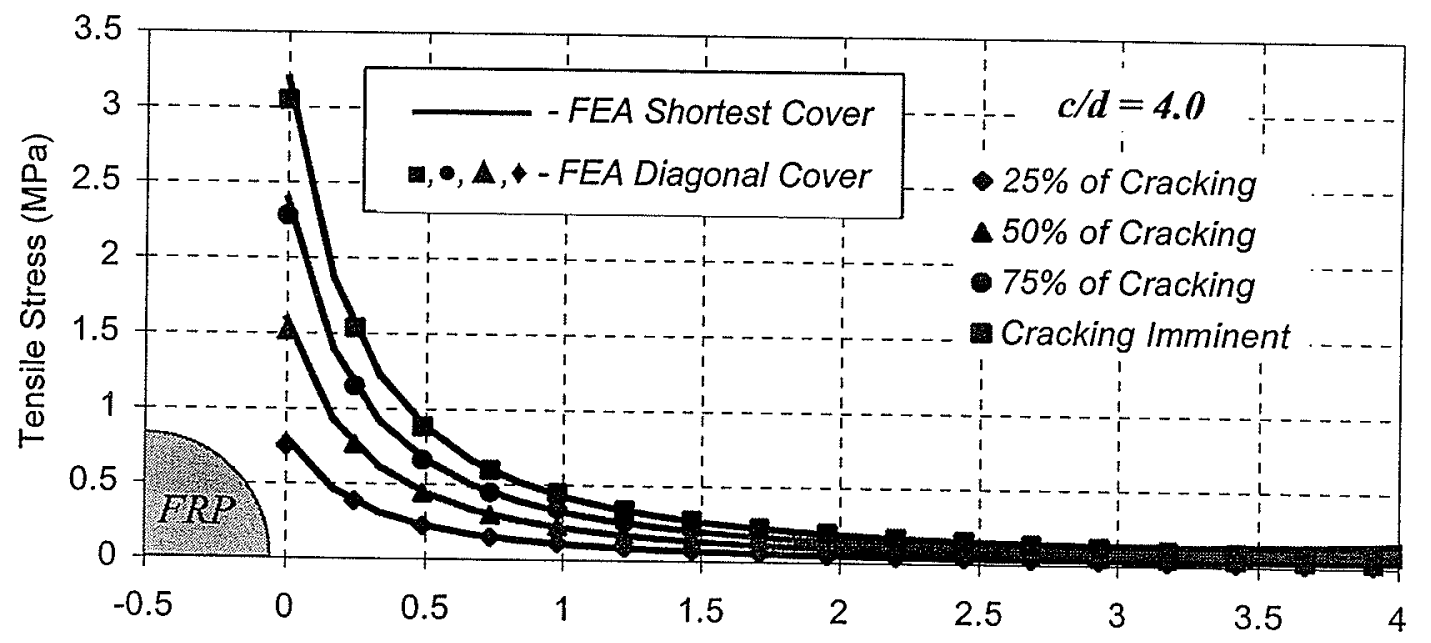

Fractional Distance Within Cover, c/d

(a) Concrete Cover to Bar Diameter Ratio $=4.0$

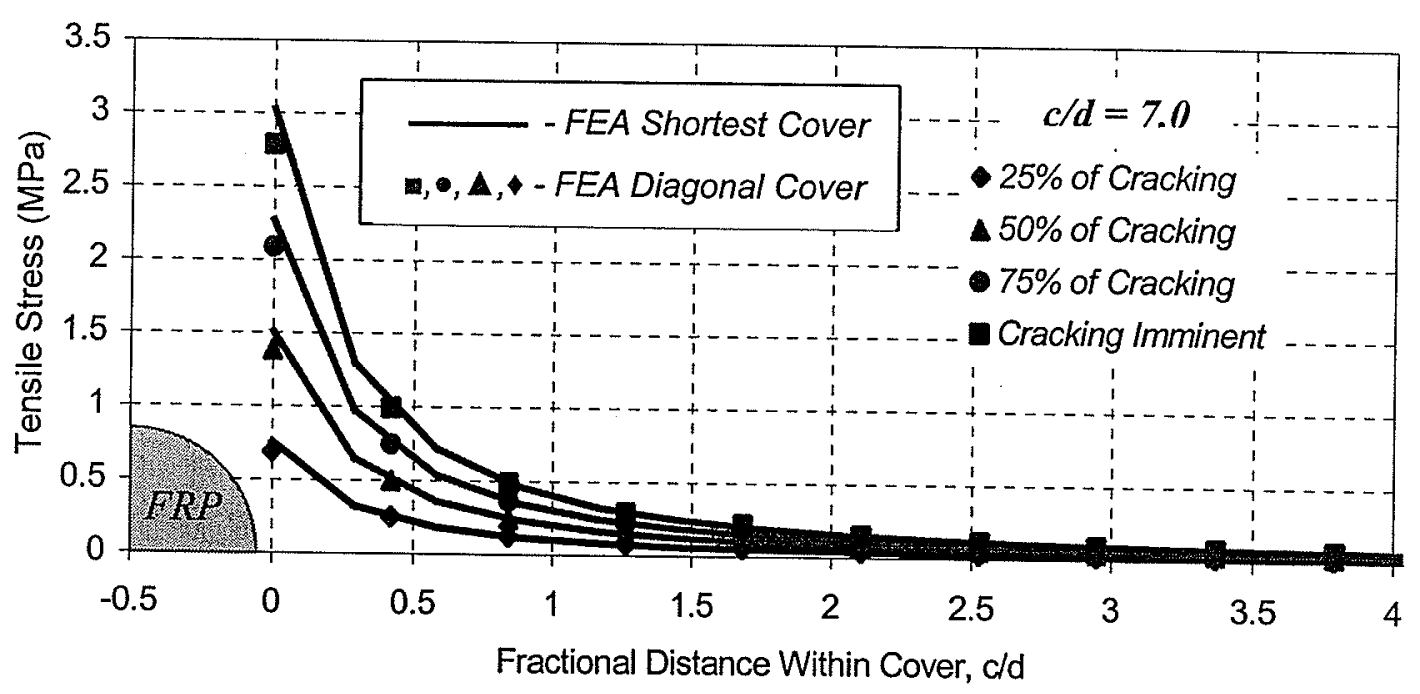

(b) Concrete Cover to Bar Diameter Ratio $=7.0$

Figure 4.11 - First Principal Stress Distribution Along Diagonal Cover

$$
\left(f_{c}^{\prime}=30 \mathrm{MPa}, E_{f}^{\top}=3,000 \mathrm{MPa}, \alpha_{\mathrm{f}}^{\top}=90 \mu \varepsilon /{ }^{\circ} \mathrm{C}\right)
$$

Larger amount of material along the diagonal does not appreciably affect the stress distribution within the concrete cover and, more specifically, stresses at the interface between the materials. Consequently, it became apparent from the finite element analysis that providing concrete cover 
values beyond the minimum requirement of CSA S806 (2002) was not efficient in relieving stresses and preventing the instigation of cracking around a swelling FRP reinforcing bar. Similar results were obtained from the linear elastic analysis, which enforces the assumption of an influence zone around the FRP reinforcement to model and investigate thermally induced stresses.

\subsection{Critical Temperature Charts}

Aside from studying the development of stresses within the concrete cover of FRP reinforced elements, the main objective of the finite element analysis was to generate critical temperature charts. As in Chapter 3 of this thesis, the charts define thermal gradients required to initiate cracking and provoke splitting of the concrete cover as a function of this parameter. Figure 4.12 illustrates critical temperature charts obtained for concrete compressive strengths of 30 and 50 $\mathrm{MPa}$, transverse CTE values of 55 and $90 \mu \varepsilon /^{\circ} \mathrm{C}$ as well as a transverse elastic modulus of 3,000 $\mathrm{MPa}$. Charts in this figure include results from the finite element analysis (FEA) as well as from the linear elastic analysis (LEA). Results from the finite element analysis in this figure are preliminary. For future research, additional work is required to elaborate results for a wider range of material properties.

When comparing results from both analyses in Figure 4.12, it became apparent that critical temperatures defining instigation of cracking within the cover of the finite element model were higher than those obtained from the linear elastic model. The difference in critical temperatures is slight, however, and is a direct consequence of the stress distribution developing within the concrete cover of the finite element model. As discussed in the previous section, the distribution was such that stresses at the interface were slightly lower than those obtained from the linear elastic analysis. Hence, higher temperatures are required to reach the tensile cracking stress within the concrete cover of the finite element model. 


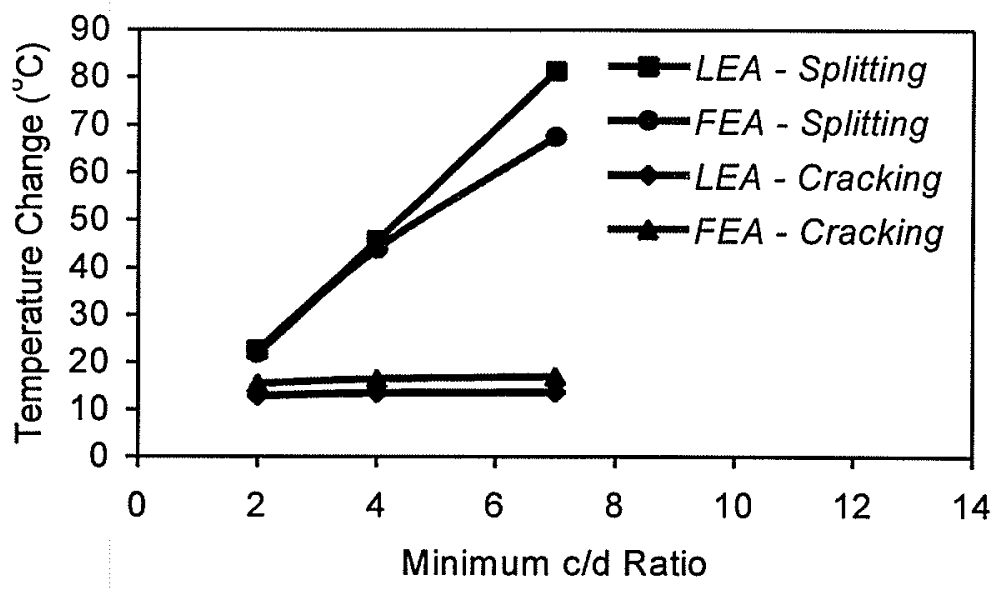

(a) $\mathrm{f}_{\mathrm{c}}=50 \mathrm{MPa}, \mathrm{E}_{\mathrm{f}}^{\top}=3,000 \mathrm{MPa}, \alpha_{\mathrm{f}}^{\top}=90 \mu \varepsilon /{ }^{\circ} \mathrm{C}$

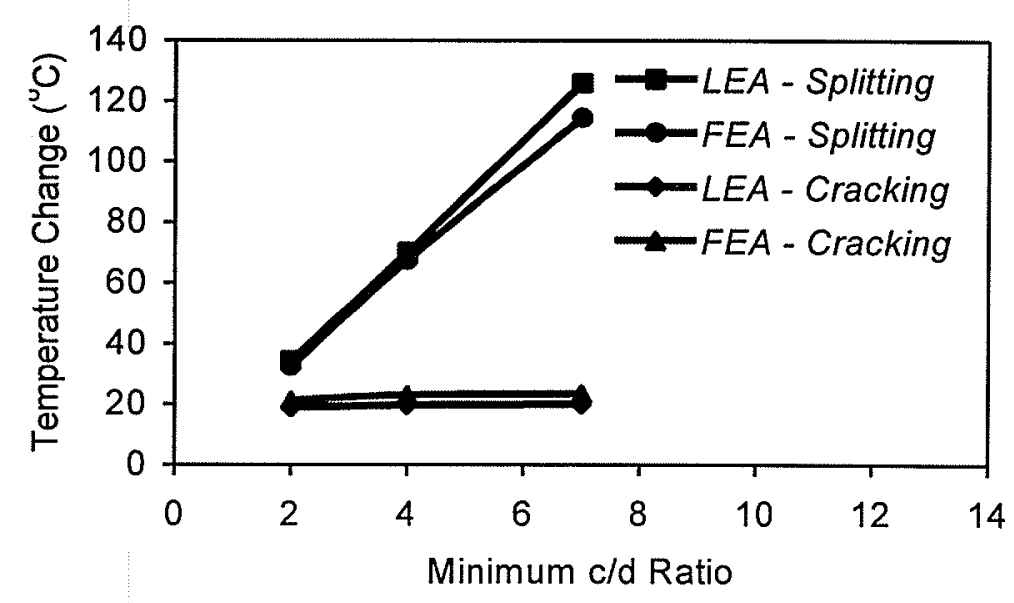

(c) $f_{c}^{\prime}=30 \mathrm{MPa}, E_{f}^{\top}=3,000 \mathrm{MPa}, \alpha_{f}^{\top}=90 \mu \varepsilon /{ }^{\circ} \mathrm{C}$

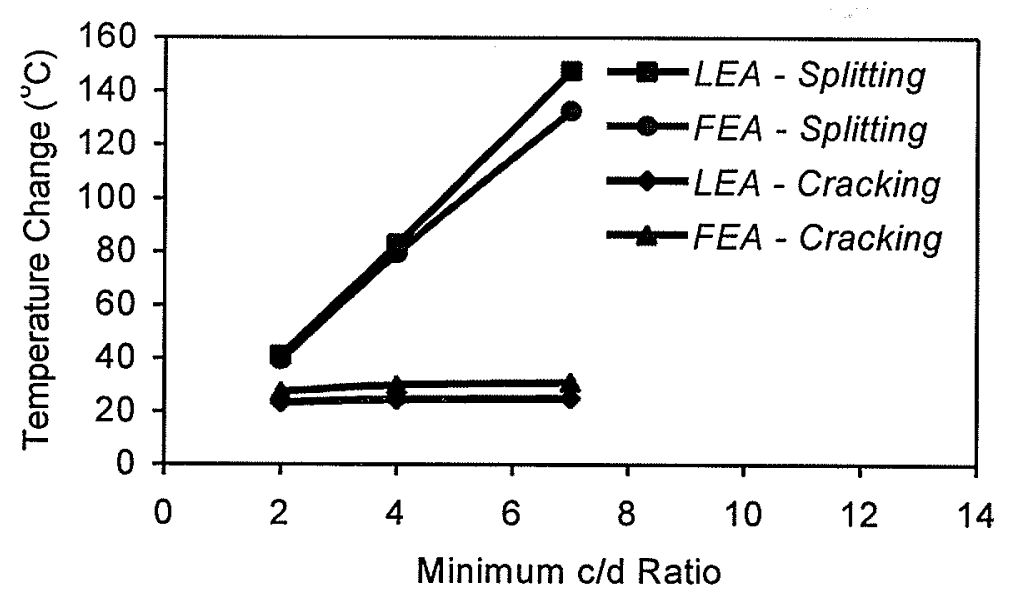

(b) $\mathrm{f}_{\mathrm{c}}=50 \mathrm{MPa}, \mathrm{E}_{\mathrm{f}}^{\top}=3,000 \mathrm{MPa}, \alpha_{\mathrm{f}}^{\top}=55 \mu \varepsilon /^{\circ} \mathrm{C}$

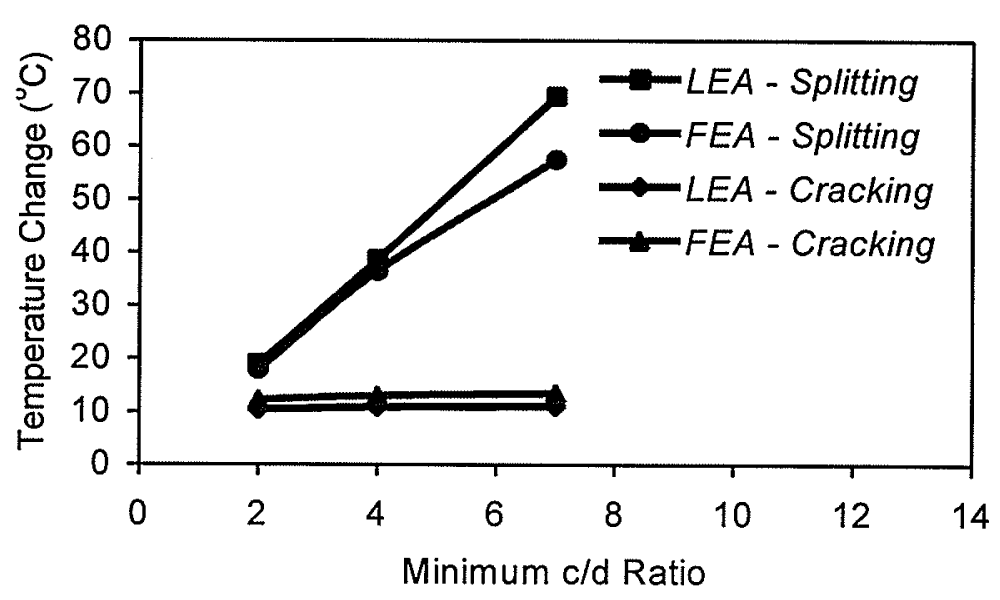

(d) $\mathrm{f}_{\mathrm{c}}^{\prime}=30 \mathrm{MPa}, \mathrm{E}_{\mathrm{f}}^{\top}=3,000 \mathrm{MPa}, \alpha_{\mathrm{f}}^{\top}=55 \mu \varepsilon /^{\circ} \mathrm{C}$ 
It also appears from Figure 4.12 that the critical temperature at splitting is very much comparable to that obtained from the linear elastic analysis. However, there exists a divergence of results at higher concrete cover values. This is attributed to the presence of excessive compressive stresses within the cover that develop during the progression of cracking. For larger cover values, a larger amount of material needs to be damaged before the splitting phenomenon occurs. As a result, compressive stresses accumulate and become excessive as damage progresses within the cover of the model. Since the model accounts for biaxial states of stress within the cover, the concrete tensile strength reduces with excessive compressive stresses and splitting occurs at a lower temperature than that anticipated by the linear elastic analysis. This aspect of the finite element analysis agrees with experimental results obtained by Aiello (1999) where lower thermal gradients were recorded at splitting for larger concrete cover values. Figure 4.13 shows complete splitting of the finite element model concrete cover. Lighter colored zones propagating along the horizontal and vertical covers represent open cracks.

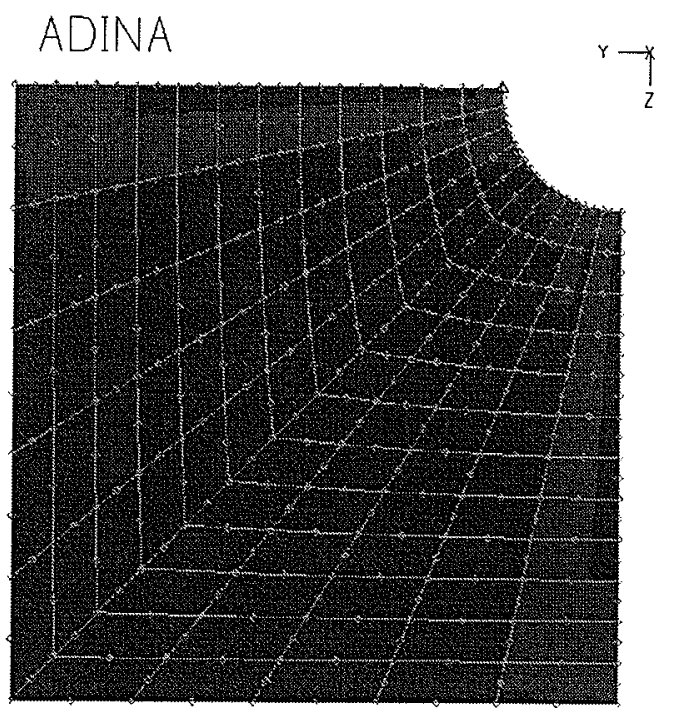

\section{Geometrical and Material Properties:}

$\begin{array}{rrr}\mathrm{f}_{\mathrm{c}}^{\prime}= & 30 \mathrm{MPa} \\ \mathrm{E}_{\mathrm{f}}^{\top}= & 3,000 \mathrm{MPa} \\ \alpha_{\mathrm{f}}{ }^{\top}= & 90 & \mu \varepsilon /{ }^{0} \mathrm{C} \\ \alpha_{\mathrm{c}} & =10 & \mu \varepsilon /{ }^{\circ} \mathrm{C} \\ \mathrm{c} / \mathrm{d}= & 2.0 & \end{array}$

Figure 4.13 - Finite Element Model Concrete Cover Splitting (130 elements, 417 nodes)

\subsection{Mesh Refinement}

Mesh refinements were also performed on the finite element model. The refinement was used to establish mesh densities required to obtain convergence and stability of results from the finite 
element analysis. Critical temperatures defining cracking and splitting of the concrete cover were therefore determined at various mesh densities until convergence. For material properties and $\mathrm{c} / \mathrm{d}$ ratios considered in the finite element analysis, results converged and stabilized once a mesh with 120 elements was reached. In light of this, critical temperature charts and results shown for the finite element analysis were obtained on the basis of a mesh described by 130 elements and 417 nodes. Typical mesh refinement results are illustrated in Figure 4.14 in terms of thermal gradients at cracking and splitting for the material properties listed.

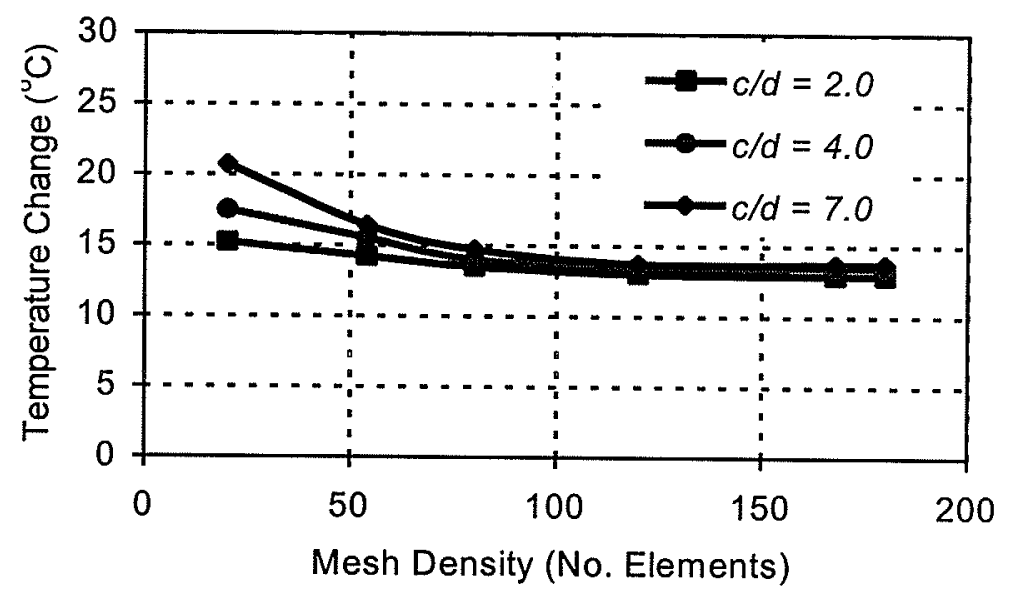

(a) Critical Temperature at Cracking

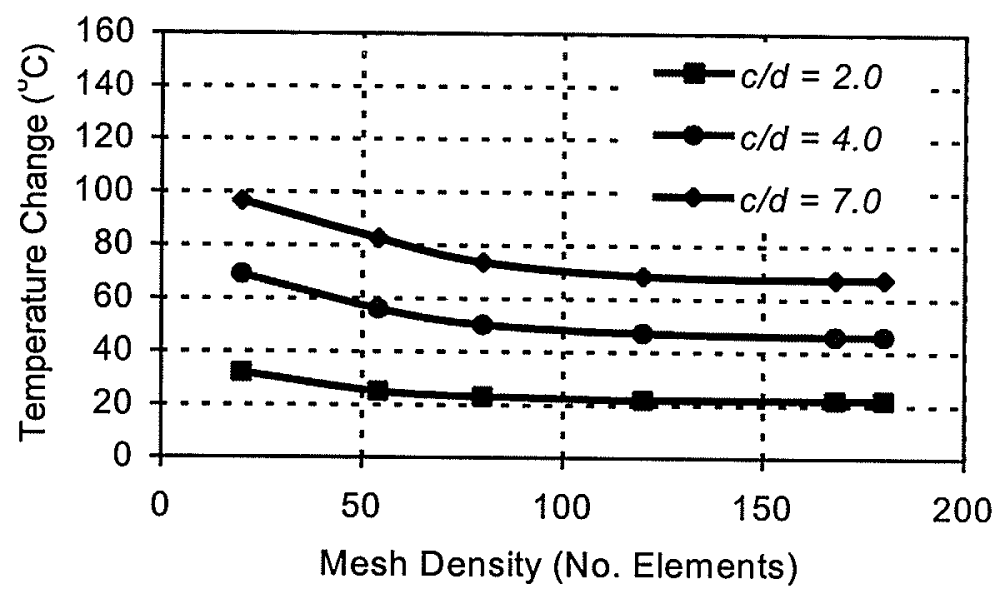

(a) Critical Temperature at Splitting

Figure 4.14 - Mesh Refinement Results $\left(f_{c}^{\prime}=50 \mathrm{MPa}, E_{f}^{\top}=3,000 \mathrm{MPa}, \alpha_{\mathrm{f}}^{\top}=90 \mu \varepsilon /{ }^{\circ} \mathrm{C}\right)$ 


\section{Chapter 5}

\section{Experimental Program}

\subsection{General}

In light of linear elastic and finite element analyses performed in this thesis, an experimental program was carried out to evaluate the effect of subjecting flexural specimens to temperature fluctuations expected in the Canadian climate. Flexural specimens in this research were provided with minimum concrete cover to investigate minimum requirements specified by CSA S806 (2002) and determine whether they are sufficient to prevent damage around FRP reinforcement and restrain deterioration of bond strength arising from temperature fluctuations considered. Flexural specimens were prestressed with CFRP and GFRP reinforcement to further increase transverse swelling and potential for damage within the concrete cover.

Aside from inspecting development of damage within the transfer region during the experimental program, bond strength of each specimen was determined by placing the load in proximity of the support. Bond strength results obtained from beams kept at ambient temperature were compared to those obtained from beams subjected to the weathering process. This comparison was used to establish whether sufficient damage could occur due to temperature fluctuations considered in the research. Several parameters were also investigated by casting a set of two control beams prior to those intended for the weathering process. 


\subsection{Control Beams}

Design manuals for the design and construction of concrete components with FRP rarely account for product variability of commercially available FRP tendons. As a result, two beams were conceived and fabricated at the University of Manitoba during the month of December 2003. The flexural behaviour of these beams was assessed with the objective of confirming the prestressing loss and development length estimates obtained from clause 10 of CSA S806 (2002) for CFRP and GFRP tendons used in the research.

\subsubsection{Dimensions}

As illustrated in Figure 5.1, the beam cross section was $90 \mathrm{~mm}$ (3.5 in.) wide and $200 \mathrm{~mm}$ deep ( $77 / 8$ in.) with a single tendon centred at $45 \mathrm{~mm}\left(1 \frac{3 / 4}{4} \mathrm{in}\right.$.) from the bottom of the beam in its casting position. Two 10M steel reinforcing bars were also provided at $40 \mathrm{~mm}$ from the top of each beam to serve as compression reinforcement. The beams were $3,600 \mathrm{~mm}$ in length. The first beam was pre-tensioned with a CFRP tendon (Aslan 200) while the second was pre-tensioned with a GFRP tendon (Aslan 100). Each tendon had a diameter of $9 \mathrm{~mm}$. Properties of these tendons were provided by the manufacturer and are discussed more fully in Chapter 3 of this thesis.

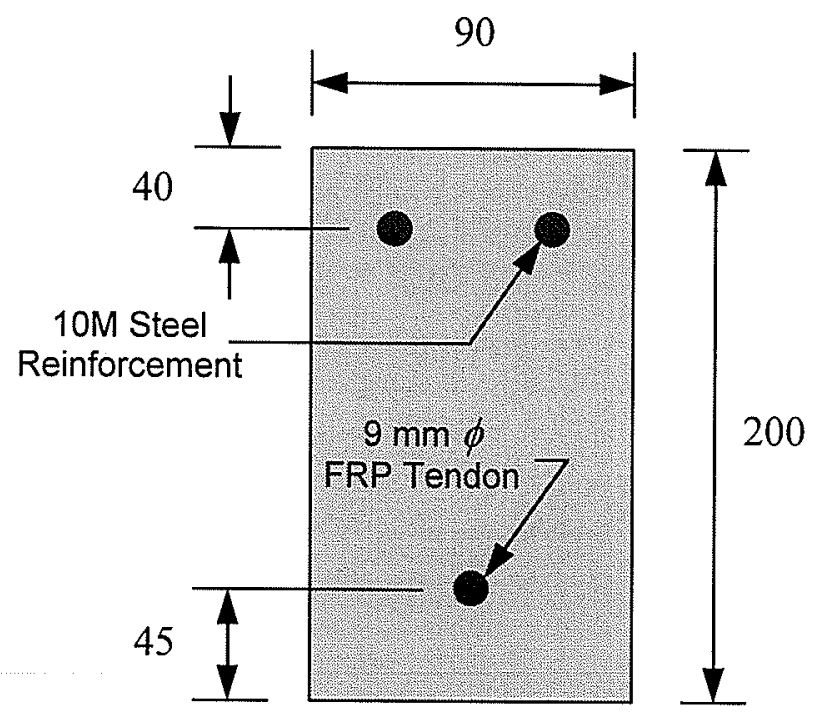

Figure 5.1 - Beam Geometry (All Dimensions in $\mathrm{mm}$ ) 


\subsubsection{Prestress Losses}

The prestressing level applied to the tendons in this research was selected on the basis of clauses 10.5.1 and 7.1.2.3 of CSA S806 (2002). For the control beams of this project, CFRP and GFRP tendons were respectively stressed to 60 percent and 30 percent of the guaranteed tensile strength provided by the manufacturer.

Prestress losses expected for the tendons used in this research were calculated on the basis of clause 10.6.2 of CSA S806 (2002) and are summarized in Table 5.1. According to the results presented in this table, an initial loss immediately after transfer of 5.1 and 1.6 percent as well as a total loss of 17.1 and 11.1 percent was expected for CFRP and GFRP tendons studied, respectively.

Table 5.1 - Estimated Prestress Losses

\begin{tabular}{|l|c|c|}
\hline \multicolumn{1}{|c|}{ LOSSES (MPa) } & CFRP (Aslan 200) & GFRP (Aslan 100) \\
\hline Relaxation Prior to Transfer & 8.4 & 1.6 \\
\hline Elastic Shortening & 54.9 & 2.3 \\
\hline Time Dependent Losses & & \\
Creep and Shrinkage & 146.0 & 20.8 \\
Relaxation & 2.8 & 0.6 \\
\hline Total Losses & 212.1 & 25.3 \\
\hline
\end{tabular}

\subsubsection{Development Length}

Based on clause 10.12 of CSA S806 (2002), the development length of bonded reinforcement is determined by adding the transfer length to the flexural bond length of the FRP tendon under consideration. The transfer length is defined as that part of the development length required to develop the prestress force in a pre-tensioned concrete element. Conversely, the flexural bond length is defined as that portion of the development length required to develop the additional 
force that takes the tendon to its full design strength. Equations that follow define these particular bond lengths.

$$
\begin{aligned}
& L_{T}=\frac{f_{f p i} d_{b}}{\alpha_{t}{f^{\prime}{ }_{c}{ }^{0.67}}^{0.6}} \\
& \text { EQ } 5.1 \\
& L_{f b}=\frac{\left(f_{f p u}-f_{f p e}\right) d_{b}}{\alpha_{f}{f^{\prime}}_{c}^{0.67}} \\
& L_{d}=L_{T}+L_{f b}
\end{aligned}
$$

Here, $f_{f p i}$ is the initial prestress in the FRP tendon immediately after release, $d_{b}$ is the bar diameter, $\alpha_{t}$ is a regression coefficient taken as 1.9 for CFRP reinforcing bars and 4.8 for CFRP strands, $f_{c}^{\prime}$ is the compressive strength of concrete, $f_{f p u}$ is the tensile strength of the FRP tendon, $f_{i p e}$ is the effective stress in the FRP tendon after allowance for all prestressing losses, $\alpha_{f}$ is a regression coefficient taken as 1.0 for CFRP reinforcing bars and 2.8 for CFRP strands, $L_{T}$ is the transfer length, $L_{f b}$ is the flexural bond length and $L_{d}$ is the development length of the FRP tendon under consideration.

Since provisions in clause 10 of CSA S806 (2002) do not report on the use of GFRP reinforcement in pretensioned concrete elements, the same bond requirements as for CFRP reinforcement were used in the determination of development length. Relevant stresses and minimum bond lengths of FRP tendons used in this research are found in Table 5.2.

\subsubsection{Concrete Stresses}

Concrete stresses occurring at the extreme fibres of the prestressed elements were calculated immediately after transfer and also after allowance for all losses. Originating from the prestressing effect and self-weight of the elements, these stresses were calculated by means of transformed section properties and relationships illustrated in Figure 5.2. 
Table 5.2 - Minimum Length of Bonded Reinforcement

(a) Tendon Prestressing Level [MPa]

\begin{tabular}{|c|c|c|}
\hline PRESTRESS LEVEL (MPa) & CFRP (Aslan 200) & GFRP (Aslan 100) \\
\hline Jacking, $f_{f p o}$ & 1,241 & 228 \\
\hline Initial, $f_{f p i}$ & 1,178 & 224 \\
\hline Effective, $f_{f p e}$ & 1,029 & 203 \\
\hline
\end{tabular}

(b) Transfer, Flexural Bond and Development Lengths [mm]

\begin{tabular}{|c|c|c|}
\hline BOND LENGTH (mm) & CFRP (Aslan 200) & GFRP (Aslan 100) \\
\hline Transfer Length, $L_{T}$ & 412 & 77 \\
\hline $\begin{array}{c}\text { Minimum Transfer Length, } \\
20 d_{b}\end{array}$ & 617 & 701 \\
\hline Flexural Bond Length, $L_{f b}$ & 678 & 371 \\
\hline Development Length, $L_{d}$ & 1,295 & 1,072 \\
\hline
\end{tabular}

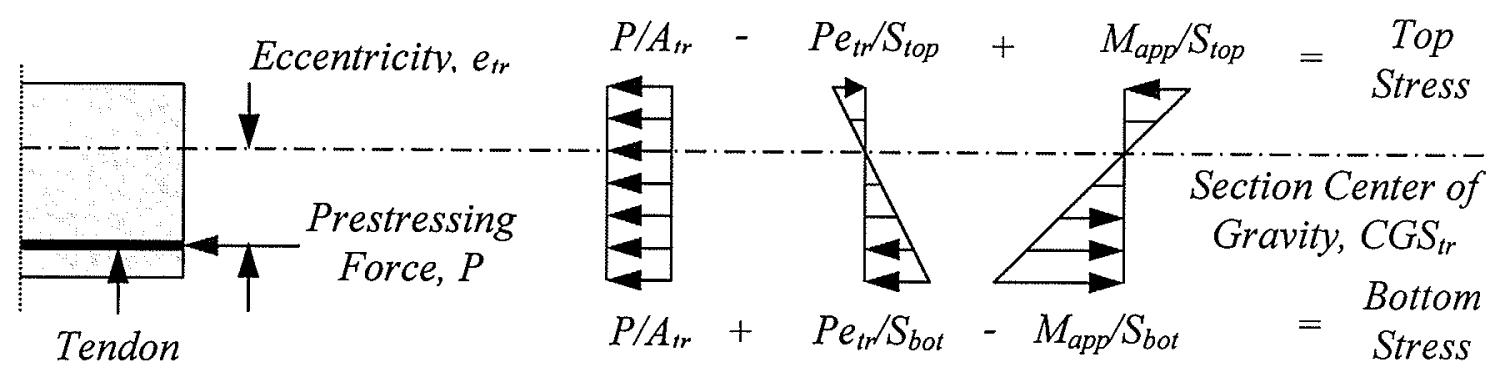

Figure 5.2 - Calculation of Concrete Stresses due to Specified Loads

Stresses were checked at all critical locations along the length of the beams, which includes the extremities of the flexural member as well as the midspan condition. While stresses at the extremities of the member govern the design immediately after transfer, stresses at midspan control the design after allowance for all prestress losses. A summary of all concrete stresses can be found in Table 5.3.

From Table 5.3(a), tension in the top fibres of the CFRP prestressed beam at release is beyond allowable stresses recommended by clause 10.4 of CSA S806 (2002). In light of excessive 
stresses, the code specifies that bonded reinforcement should be provided in the tensile zone to resist the total tensile force $N_{c}$ in the concrete computed on the basis of an uncracked section.

Table 5.3 - Critical Concrete Stresses [MPa]

(a) CFRP Tendon

\begin{tabular}{|c|c|c|c|c|c|c|}
\hline & \multicolumn{4}{|c|}{$\begin{array}{l}\text { Stresses@ Release } \\
\qquad(P=P)\end{array}$} & \multicolumn{2}{|c|}{$\begin{array}{l}\text { Long-Term Stresses } \\
(P=P)\end{array}$} \\
\hline & \multicolumn{2}{|c|}{ Support } & \multicolumn{2}{|c|}{ Midspan } & \multicolumn{2}{|c|}{ Midspan } \\
\hline & Top & Bottom & Top & Bottom & Top & Bottom \\
\hline$P / A_{t r}$ & 5.01 & 5.01 & 5.01 & 5.01 & 4.33 & 4.33 \\
\hline$P e_{t r} / S_{t r}$ & -8.35 & 8.81 & -8.35 & 8.81 & -7.21 & 7.60 \\
\hline$M_{s w} / S_{t r}$ & - & - & 0.24 & -0.25 & 0.24 & -0.25 \\
\hline Stresses & -3.34 & 13.82 & -3.10 & 13.57 & -2.64 & 11.65 \\
\hline Allowable & $\begin{array}{c}-2.96 \\
\left(-0.5 f_{c i}^{\prime 0.5}\right)\end{array}$ & $\begin{array}{l}21.00 \\
\left(0.6 f_{c i}^{\prime}\right)\end{array}$ & $\begin{array}{c}-1.48 \\
\left(-0.25 f_{c i}^{0.5}\right)\end{array}$ & $\begin{array}{l}21.00 \\
\left(0.6 f_{c i}^{\prime}\right)\end{array}$ & $\begin{array}{c}30 \\
\left(0.6 f_{c}^{\prime}\right)\end{array}$ & $\begin{array}{c}-3.54 \\
\left(-0.5 f_{c}^{, 0.5}\right)\end{array}$ \\
\hline Check & $\mathrm{HIGH}$ & $O K$ & $H I G H$ & OK & $O K$ & OK \\
\hline
\end{tabular}

(b) GFRP Tendon

\begin{tabular}{|c|c|c|c|c|c|c|}
\hline & \multicolumn{4}{|c|}{$\begin{array}{l}\text { Stresses @ Release } \\
(P=P))\end{array}$} & \multicolumn{2}{|c|}{$\begin{array}{l}\text { Long-Term Stresses } \\
\qquad(P=P)\end{array}$} \\
\hline & \multicolumn{2}{|c|}{ Support } & \multicolumn{2}{|c|}{ Midspan } & \multicolumn{2}{|c|}{ Miespan } \\
\hline & Top & Bottom & Top & Bottom & TOP & Bottom \\
\hline$P / A_{t r}$ & 0.90 & 0.90 & 0.90 & 0.90 & 0.78 & 0.78 \\
\hline$P e_{t r} / S_{t r}$ & -1.49 & 1.53 & -1.49 & 1.53 & -1.33 & 1.30 \\
\hline$M_{s w} / S_{t r}$ & - & - & 0.24 & -0.25 & 0.24 & -0.25 \\
\hline Stresses & -0.59 & 2.43 & -0.35 & 2.18 & -0.31 & 1.83 \\
\hline Allowable & $\begin{array}{c}-2.96 \\
\left(-0.5 f_{c i}^{\prime 0.5}\right)\end{array}$ & $\begin{array}{l}21.00 \\
\left(0.6 f_{c i}^{\prime}\right)\end{array}$ & $\begin{array}{c}-1.48 \\
\left(-0.25 f_{c i}^{0.5}\right)\end{array}$ & $\begin{array}{l}21.00 \\
\left(0.6 f_{c i}^{\prime}\right)\end{array}$ & $\begin{array}{c}30 \\
\left(0.6 f_{c}^{\prime}\right)\end{array}$ & $\begin{array}{c}-3.54 \\
\left(-0.5 f_{c}^{30.5}\right)\end{array}$ \\
\hline Check & OK & OK & $O K$ & OK & OK & OK \\
\hline
\end{tabular}


Clause 18.4.1.2 of CSA A23.3 (1994) limits the stress in bonded steel reinforcement to 50 percent of yielding. Noticing that the stress distribution in the section is linear in tension, the stresses in top steel reinforcement already present in the section can be determined and compared to this limiting value. On the basis of stresses listed in Table 5.3(a), transformed section properties and Figure 5.3 defining the tensile force $N_{c}$, the top steel stresses were respectively estimated at 39 and 36 percent of yielding at the ends and midspan. Bonded reinforcement provided in the section is therefore adequate to carry the excessive stresses caused by the prestressing application.

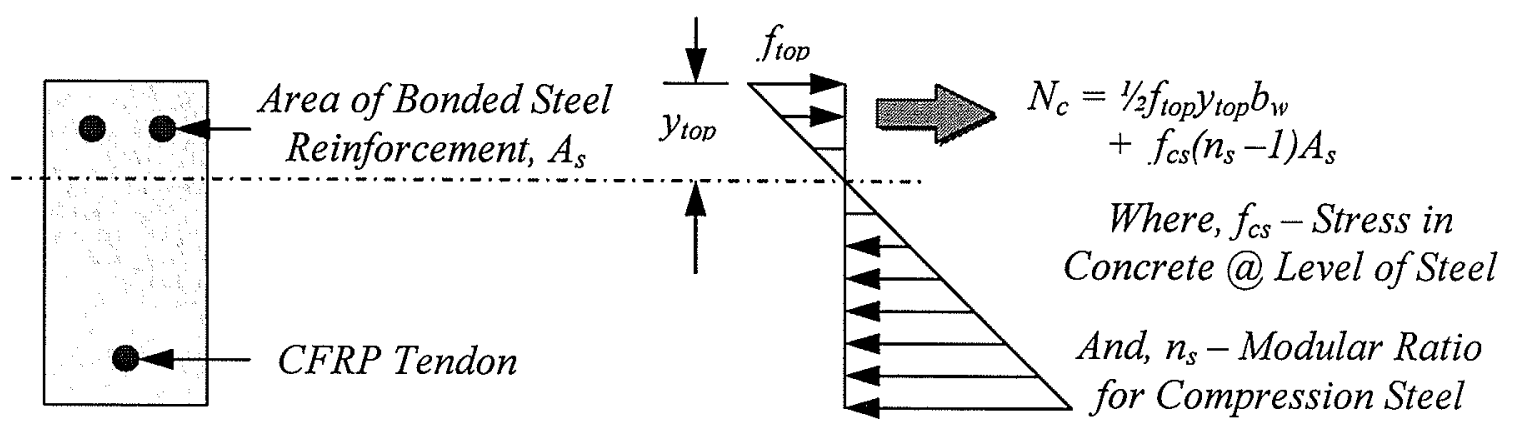

Figure 5.3 - Control of Cracking at Transfer

\subsubsection{Flexural Capacity}

All beams in this project are under-reinforced with FRP reinforcement. Failure is therefore instigated by rupture of the composite reinforcement with the corresponding concrete strain at the extreme compressive fibre $\varepsilon_{c}$ being less than the ultimate concrete compressive strain $\varepsilon_{c u}$. In light of this condition, the traditional rectangular block can no longer be used to describe the distribution of compressive stresses in the concrete zone at failure and a constitutive relationship needs to be adopted for determining the capacity of the section. 


\subsubsection{Material Constitutive Relationships}

Many mathematical models and empirical formulas are available for numerical approximation of the stress-strain diagram of concrete in compression for the case of failures initiated by rupture of the reinforcement. The concrete constitutive relationship used in this research is based on what is referred to as the TTJ model. The model was initially proposed by Popovics (1970) and later modified by Thorenfeldt, Tomaszewicz, and Jensen (1987).

Before the capacity of the section can be determined, a constitutive relationship is also required for describing the stress-strain behaviour of FRP reinforcement. As discussed and illustrated in Chapter 2 of this thesis, the stress-strain relationship of FRP reinforcement is linear elastic until failure. The failure occurs suddenly by rupture of the composite and absence of yielding typically observed in steel reinforcement. Stresses in the composite reinforcement can therefore be written as the product of the strain and elastic modulus at any loading stage prior to failure.

\subsubsection{Moment Resistance}

The moment resistance can be established on the basis of concrete and FRP models described and discussed in the preceding section. The calculation mainly relies on integration of the compressive stress-strain curve for concrete. The integration generates a rectangular stress block with factors, $\alpha$ and $\beta$, preserving the compressive force magnitude and point of application in the concrete. This compressive force is defined by EQ 5.4 and illustrated in Figure 5.4 along with the strain and stress distributions at ultimate.

$$
C_{c}=\alpha \beta f^{\prime}{ }_{c} c_{N A} b_{w} \quad \text { EQ } 5.4
$$

Recalling that beams in this research are under-reinforced, the tension force illustrated in Figure 5.4 and defined in EQ. 5.5 can be determined by noticing that the total strain in the tendon at 
failure is equal to the ultimate tendon strain $\varepsilon_{\text {frpu. }}$. The depth of neutral axis $c_{N A}$ can then be determined by considering force equilibrium and further noticing that the strain in the compressive reinforcement $\varepsilon_{s}^{\prime}$ can be written in terms of the concrete compressive strain $\varepsilon_{c}$.

$$
T_{f r p}=\varepsilon_{f r p u} E_{f r p} A_{f r p} \quad \text { EQ } 5.5
$$

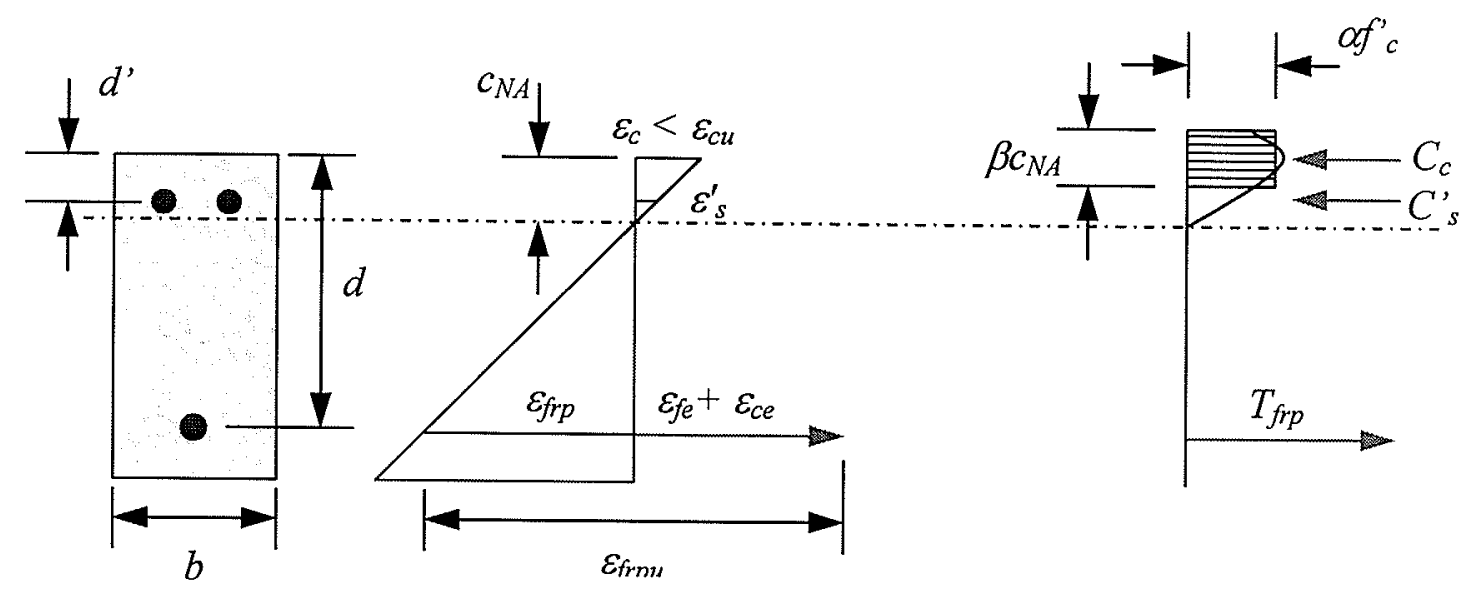

(a) Strain Distribution

(b) Stress Distribution

Figure 5.4 - Strain and Stress Distribution at Ultimate

Once the forces in the reinforcing layers and concrete compressive zone are defined, the moment resistance of the section can be determined by taking moments about the tendon. The moment resistance of each control beam was calculated on the basis of EQ 5.6 and listed in Table 5.4.

$$
M_{R}=C_{c}\left(d-\frac{\beta c}{2}\right)+C_{s}^{\prime}\left(d-d^{\prime}\right) \quad \text { EQ } 5.6
$$

Table 5.4 - Moment Resistance

\begin{tabular}{|c|c|c|c|}
\hline \multicolumn{2}{|c|}{ CFRP (Aslan 200) } & \multicolumn{2}{|c|}{ GFRP (Aslan 100) } \\
\hline Test \#1 & Test \#2 & Test \#1 & Test \#2 \\
\hline \multicolumn{2}{|c|}{18.6} & \multicolumn{2}{|c|}{9.0} \\
\hline
\end{tabular}

Moment Resistance, $M_{R}[\mathrm{kN}-\mathrm{m}]$ 


\subsubsection{Testing Procedure}

All beams were tested under single point loading. The load was placed in proximity of the support within the development length of each tendon to cause a bond failure typically characterized by slippage of the reinforcement. As previously stated, all beams were underreinforced and designed to fail in tension. In the event of superior bond performance, FRP tendons will reach their full design capacity at ultimate and lower development lengths will be determined on the basis of electrical strain gauges superficially placed on the surface of reinforcement.

The design allowed for development length tests at each end of the beams. As illustrated in Figure 5.5, the beams overhang one support during both development length tests. The concept of a beam with overhang was introduced to restrain the extent of flexural damage within the span intended for the second test. Supports were also brought closer for the second test to maintain the damaged segment outside of the testing span.

\subsubsection{Shear Reinforcement}

A shear analysis was performed for each beam on the basis of the procedure shown in Figure 5.5 and the corresponding machine load at failure. Due to its higher capacity, the beam prestressed with CFRP was provided with shear reinforcement along its entire length. On the other hand, due to the lower capacity of the beam prestressed with GFRP, shear reinforcement was not required but provided in critical regions to prevent excessive damage of the web during the first development test. This allowed for the behaviour of the beam to remain unaffected during the second test.

Due to the relatively small beam width, $6 \mathrm{~mm}$ diameter stirrups were used as shear reinforcement in both beams. Stirrups were tied to the compression reinforcement and spaced at $100 \mathrm{~mm}$ on 
centre for a total of 36 in the CFRP prestressed beam and 15 in the GFRP prestressed beam. The reinforcing scheme is shown in Figure 5.6 with assembly of a reinforcing cage enclosing one of the tendons illustrated in Figure 5.7.

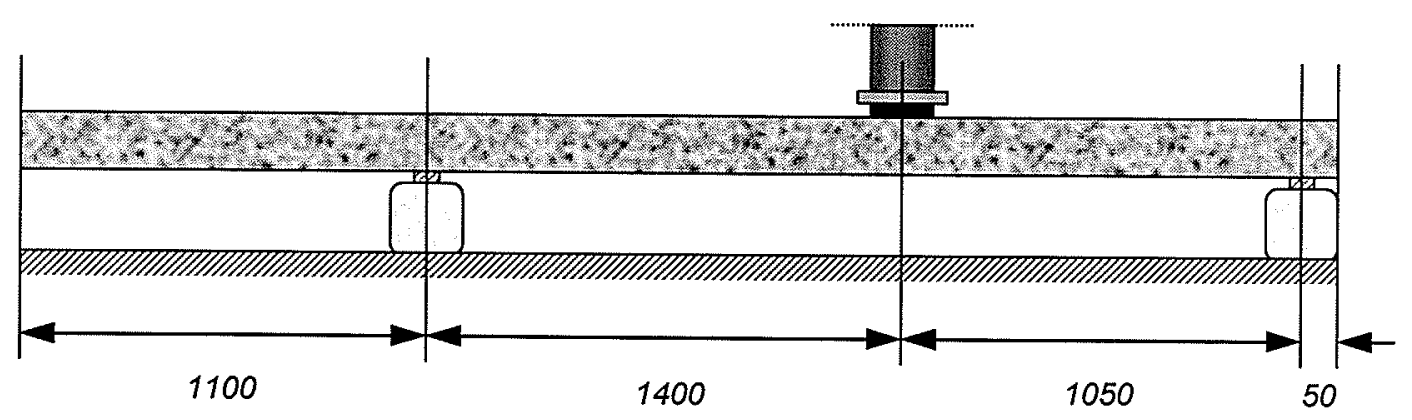

(a) First Development Length Test (Test \#1)

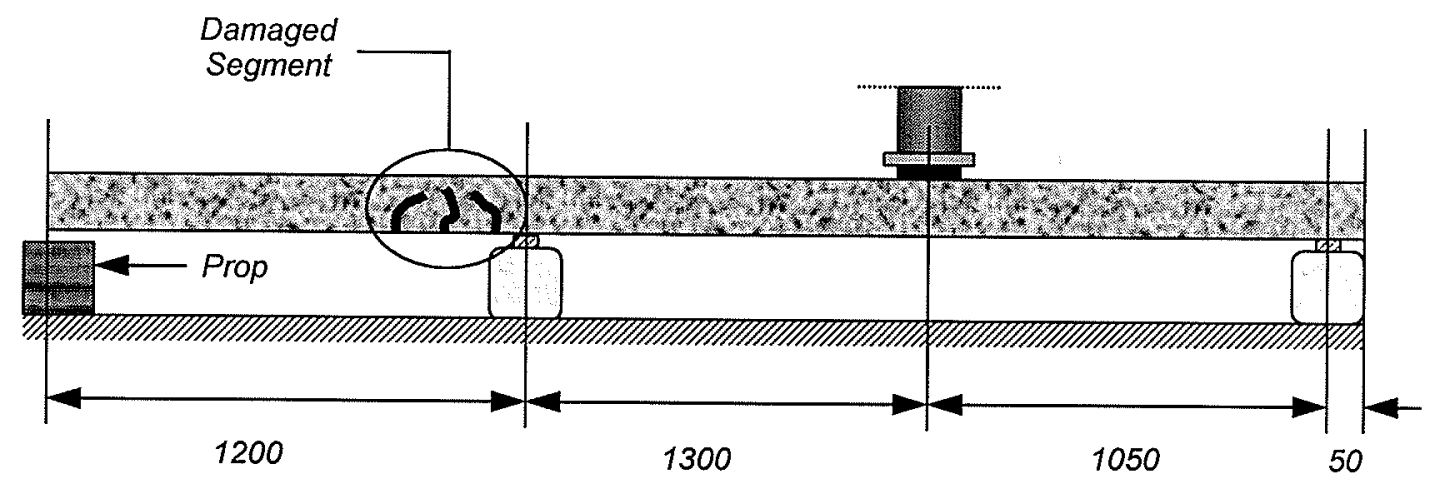

(b) Second Development Length Test (Test \#2)

Figure 5.5 - Testing Procedure

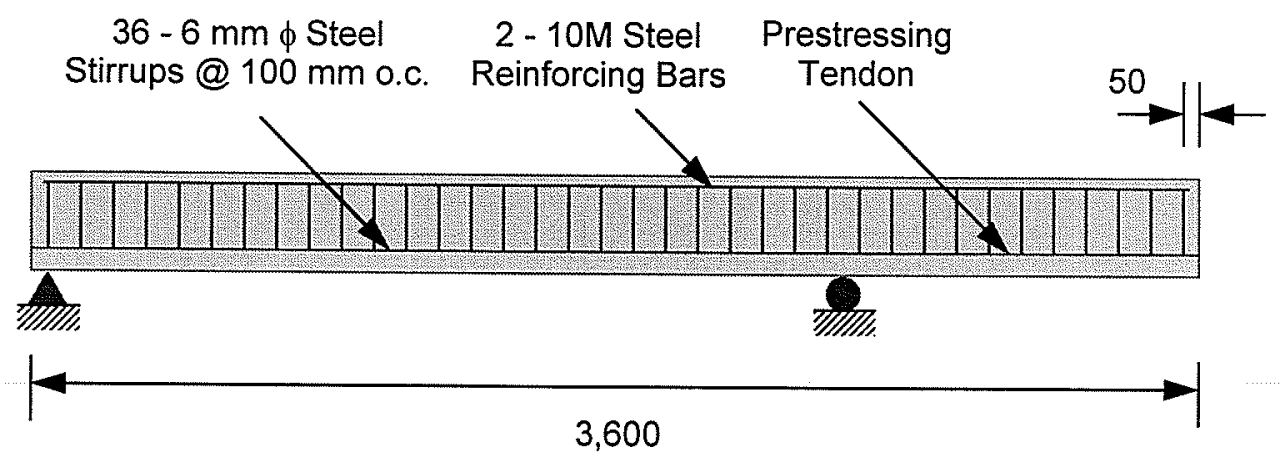

(a) CFRP Prestressed Beam - 36 Stirrups 


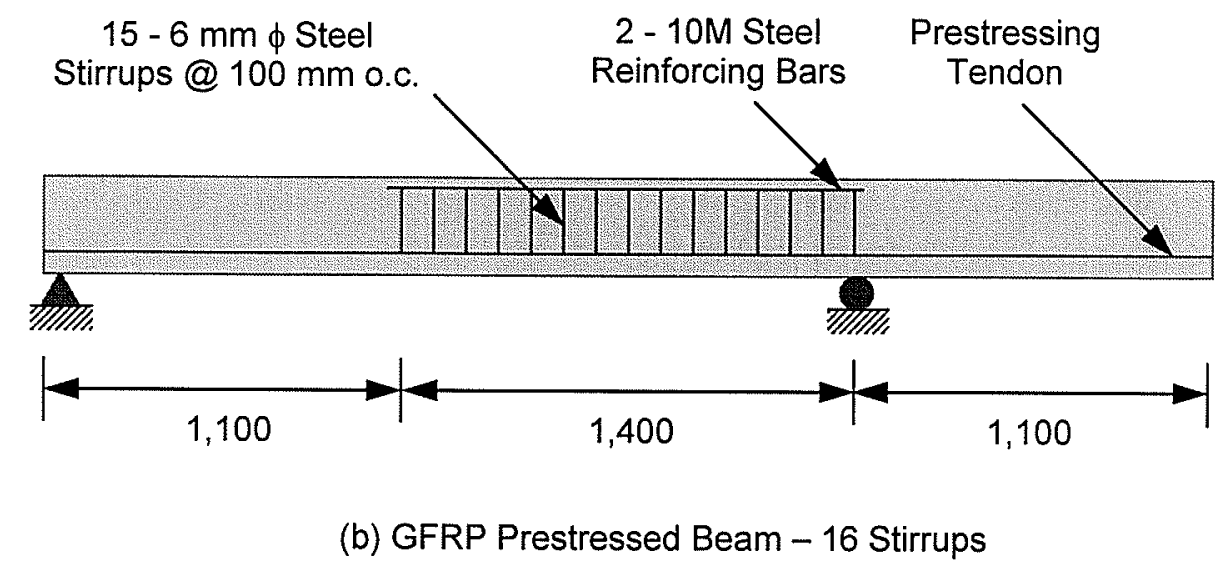

Figure 5.6 - Shear Reinforcing Scheme for Control Beams

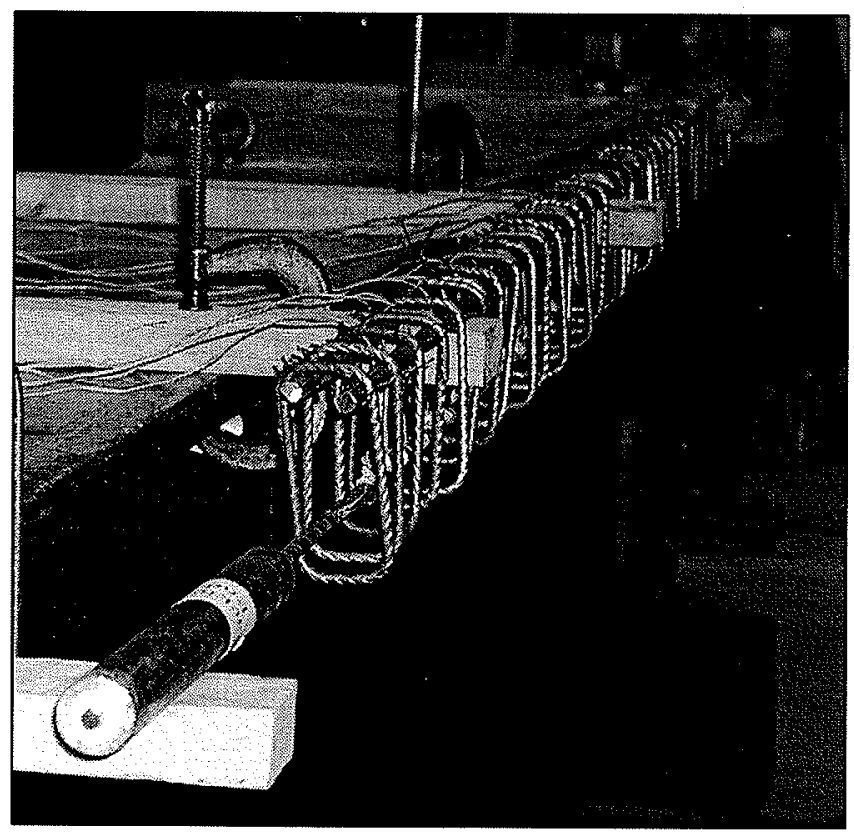

Figure 5.7 - Reinforcing Cage Assembly

\subsubsection{Application of Prestress}

Prestressing and casting of the specimens took place at the University of Manitoba McQuade Structures Laboratory in the month of December 2003. The prestressing force was applied to the strands through the use of a hydraulic jack. The jack was calibrated using a $60 \mathrm{kip}(267 \mathrm{kN})$ testing machine to convert pressure readings into load. 
As shown in Figure 5.8, both specimens were prestressed in the same bed between the same bulkheads. Due to the higher prestressing force in the CFRP strand, a thorough analysis was performed to ensure existing bulkheads and dywidag bars used as fixtures to the structural floor had sufficient capacity under eccentric loading.

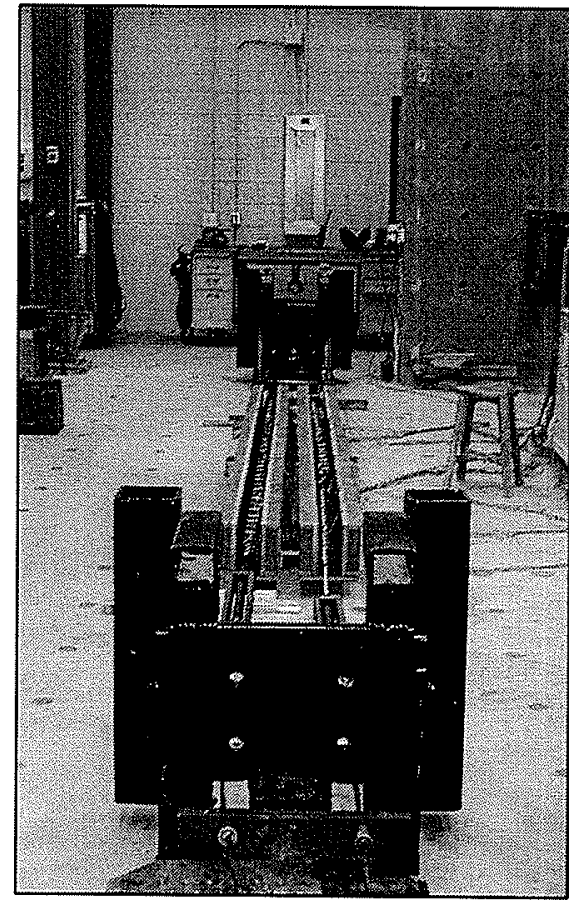

(a) Prestressing Bed

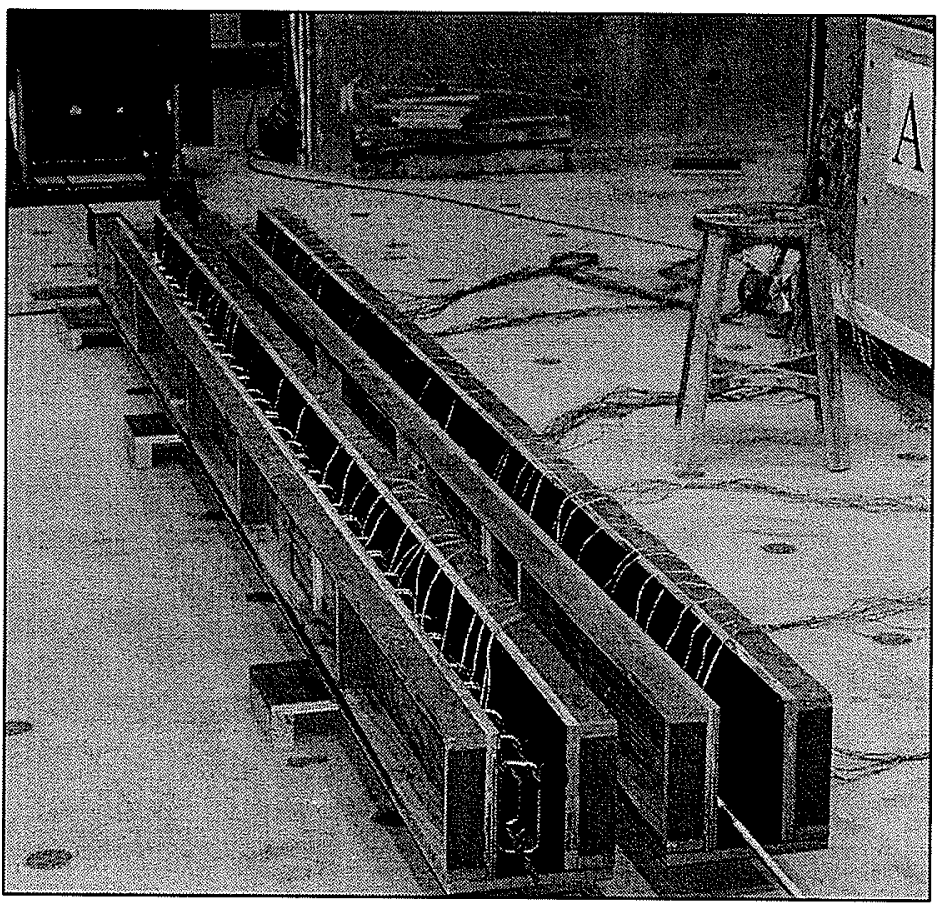

(b) Formwork Assembly Before Application of Prestress

Figure 5.8 - Prestressing Layout

The sides and bottom of the formwork were assembled around the strand and reinforcing cage prior to the prestressing application. A total of 26 strain gauges were connected to a data acquisition (DAQ) system to monitor the strand elongations during the prestressing application in relation to the applied hydraulic pressure. After the prestressing application on December $17^{\text {th }}$ 2003 , edges of the formwork were closed with end plates for casting the following day.

Due to the composition of FRP reinforcement and its consequent frailty in the transverse direction, the use of chucks as in prestressed steel applications was inadequate. A coupler 
mechanism was therefore designed to transfer prestress to the strands. Aside from having distinct dimensions to accommodate the geometrical and mechanical properties of tendons used in the current study, the mechanism was conceptually similar to that used in a previous research involved with prestressing of FRP reinforcement at the University of Manitoba (Banthia, 2003).

\subsubsection{Design and Testing of Steel Couplers}

Each coupler consisted of two longitudinal steel plates welded to transverse steel plates at both ends. Each end was fitted with a hole, the first of which allows the insertion of a $13 \mathrm{~mm}$ diameter 7-wire low relaxation steel strand gripped with a chuck. The second hole allows placement of the $35 \mathrm{~mm}$ diameter anchor bonded to the end of the FRP tendon. A spacer was required and fabricated to prevent the anchor from sliding out of the second hole of the coupler during application of prestress. Figure 5.9 shows a detailed drawing and picture of the coupler assembly.

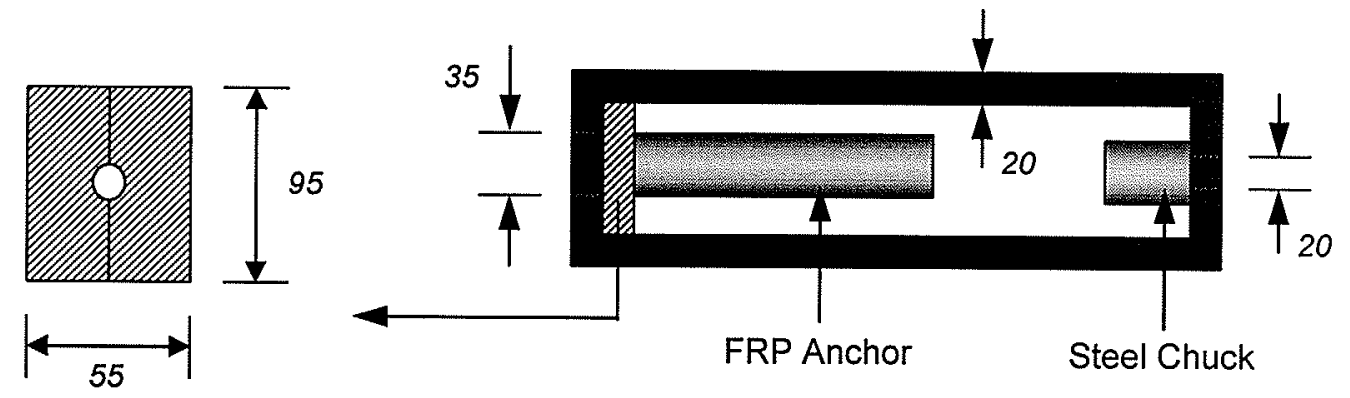

(a) Dimensions

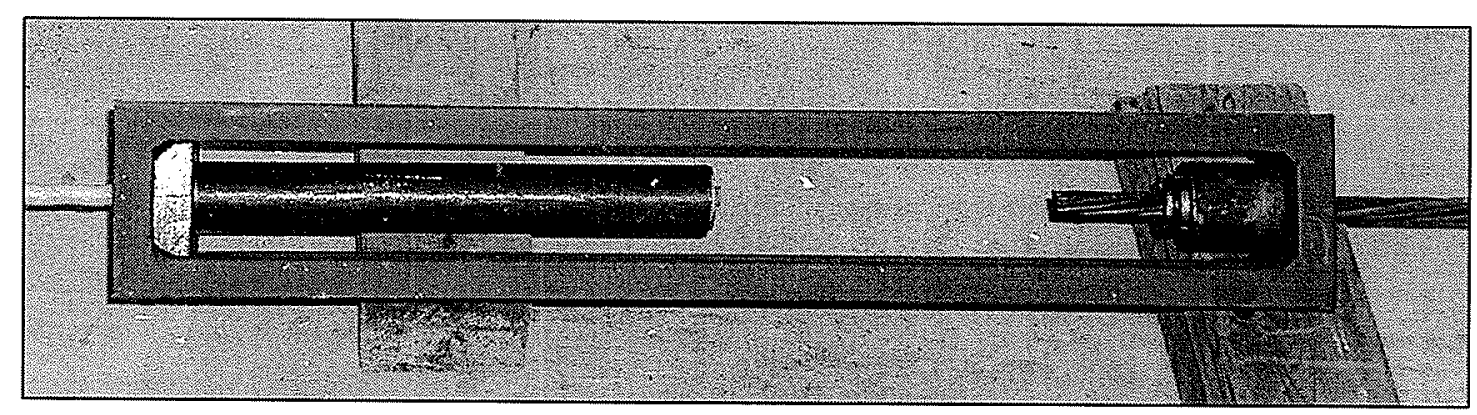

(b) Assembly During Application of Prestress

Figure 5.9 - Coupler Mechanism and Assembly 
As illustrated by Figure 5.10, a coupler was tested in tension to ensure sufficient strength was available for application of prestress. Designed with a capacity exceeding the prestressing force required for the study, the coupler was capable of sustaining a load of $35 \mathrm{kips}$ during the tension test. This load represents twice the maximum prestressing force that is to be carried by the CFRP tendon.

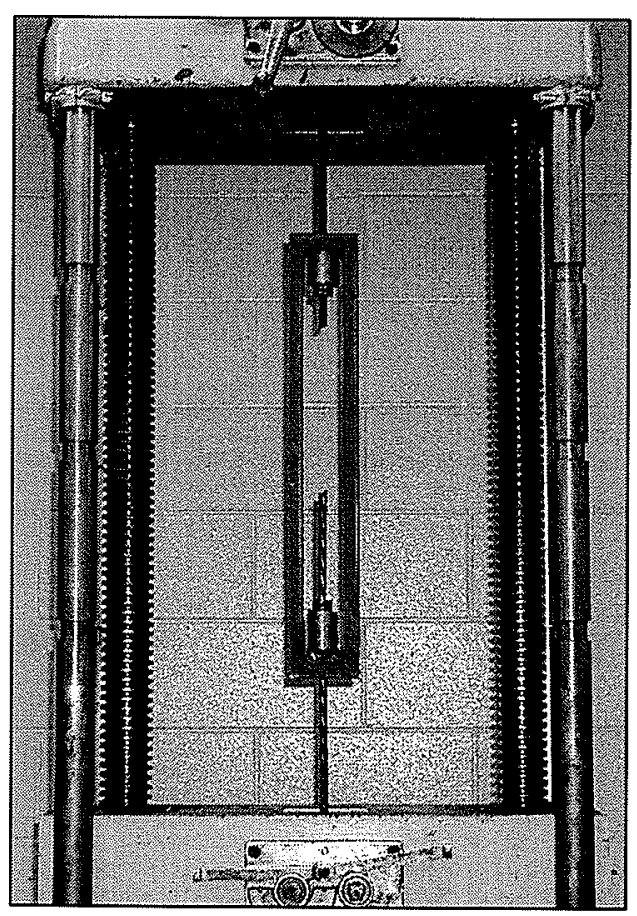

Figure 5.10 - Coupler Tension Test

\subsubsection{Prestress Monitoring}

During the application of prestress, strains were closely monitored with a total of 37 electrical resistance strain gauges distributed along the length of each tendon and two DAQ systems, which are illustrated in Figure 5.11(a). The procedure allows verification of the prestressing load applied by the hydraulic jack on the basis of elongation.

Prestressing was sustained during a total of 23 days and released by cutting the steel strands at the jacking end of the prestressing bed with a blowtorch. The procedure is illustrated in Figure 
5.11(b) with steel strand wires individually cut to provide a gradual and efficient transfer of the prestressing force to the structural elements.

Strains were also recorded prior to and after release of prestress to confirm the estimated losses and transfer lengths. Figure 5.12 illustrates the variation of strain with time after pretensioning, confirming the loss of prestress due to sustained load as well as elastic shortening of the section at the time of release. On the basis of these strain readings, a total prestress loss of 7.3 and 6.7 percent were respectively estimated for the CFRP and GFRP tendons immediately after release. Similarly, total prestress losses were estimated at 12.3 and 9.8 percent for the CFRP and GFRP tendons, respectively. These values are slightly different than those predicted using clause10.6.2 of CSA S806 (2002). Although total losses were lower than anticipated, initial losses were noticeably larger than that predicted for both tendons. In reference to Figure 5.12 , it is apparent that relaxation has greatly contributed to the digression between predicted and measured values for initial loss in any of the tendons. Moreover, the steel strand and coupler mechanism used to apply the prestress force to the FRP tendons will also cause additional losses when considering the use of steel chucks. These frictional losses contribute to total losses recorded between the application and release of prestress.

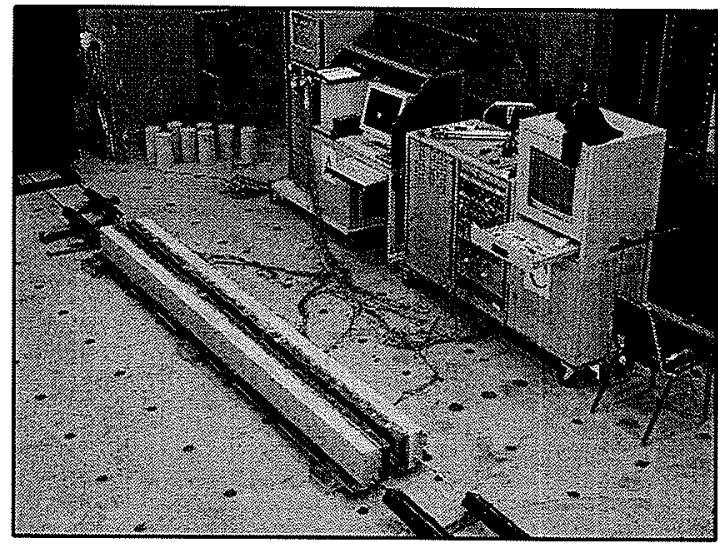

(a) DAQ System for Strain Monitoring

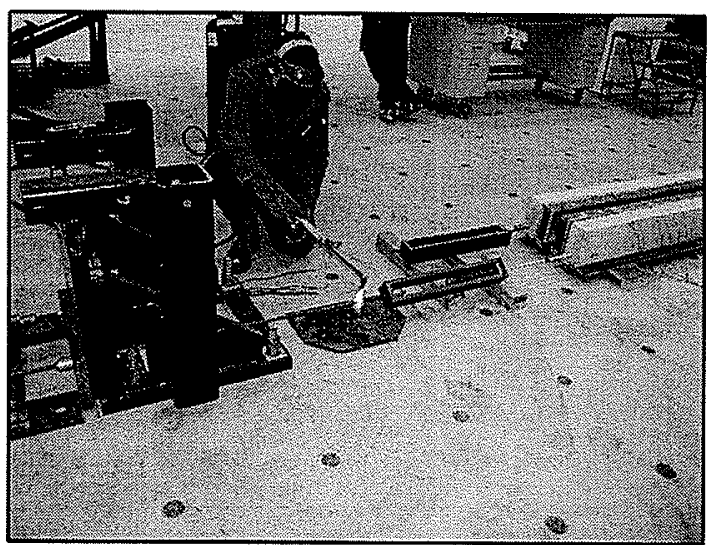

(b) Release of Prestress

Figure 5.11 - Prestressing Loss and Transfer Length Confirmation 


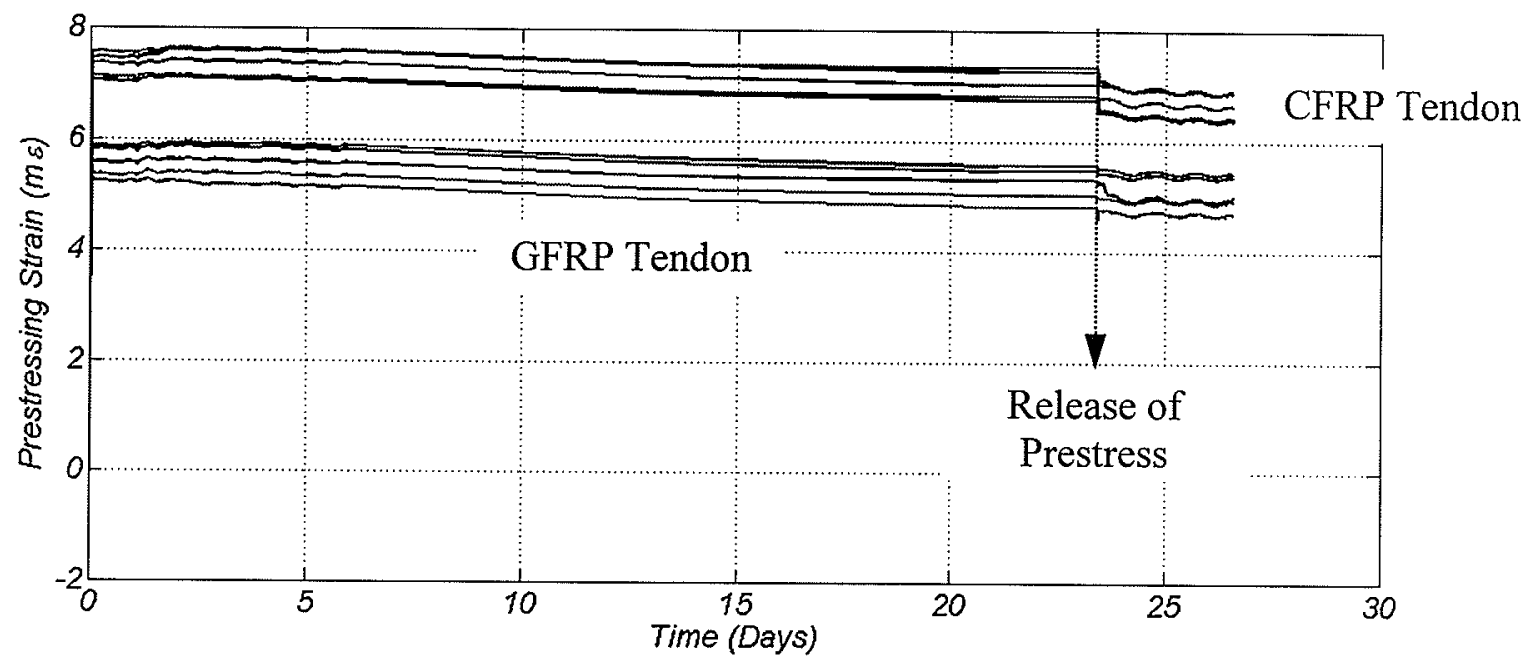

Figure 5.12 - Tendon Strain Variation with Time

To ensure reliability of strain readings at the time of release, transfer lengths were also estimated with Demec points placed on the surface of the beams at the prestressing level. All Demec points were cemented to the beams using Devcon 5-minute ${ }^{\circledR}$ epoxy with the sides of the formwork removed as shown in Figure 5.13.

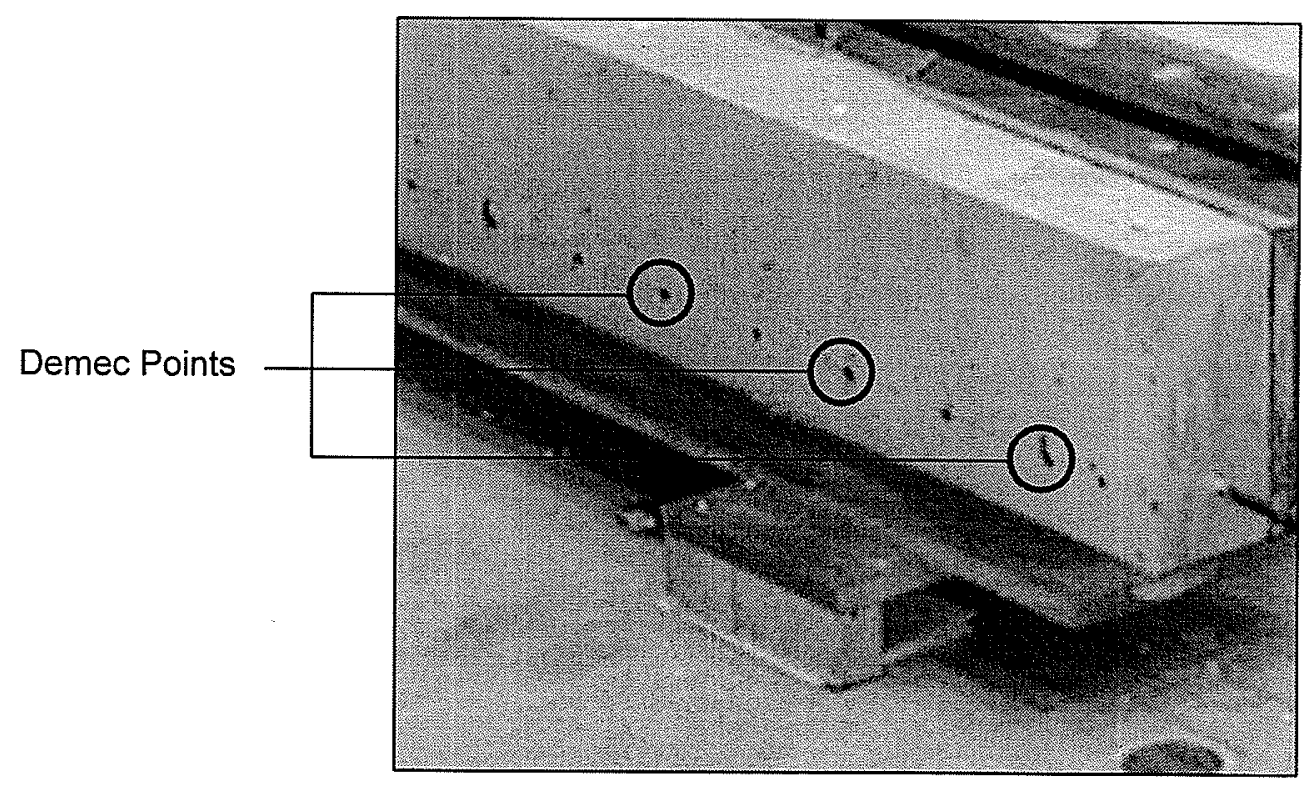

Figure 5.13 - Demec Points at Prestressing Level 
Figure 5.14(a) illustrates the strains in the tendons remaining after the release of prestress. From these curves, the length required to develop the prestressing force in the CFRP and GFRP tendons was estimated as $316 \mathrm{~mm}$ and $143 \mathrm{~mm}$ respectively. These values are confirmed by Demec point readings shown in the Figure 5.14(b), which represent elastic shortening of the section at release.

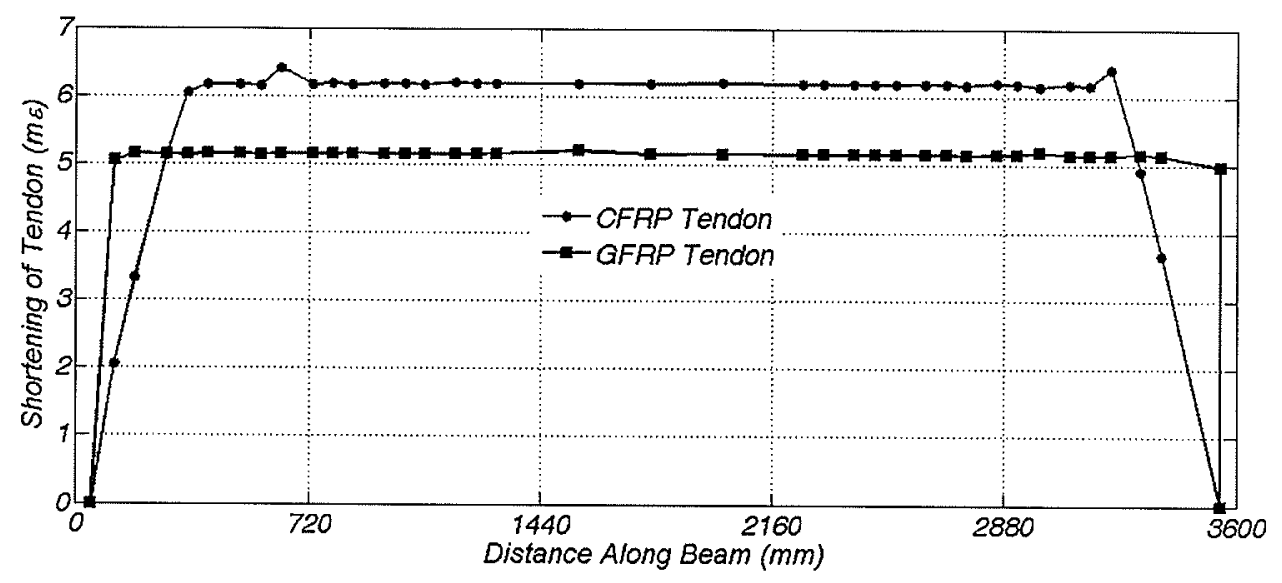

(a) Elastic Shortening of Tendon - Strain Gauges

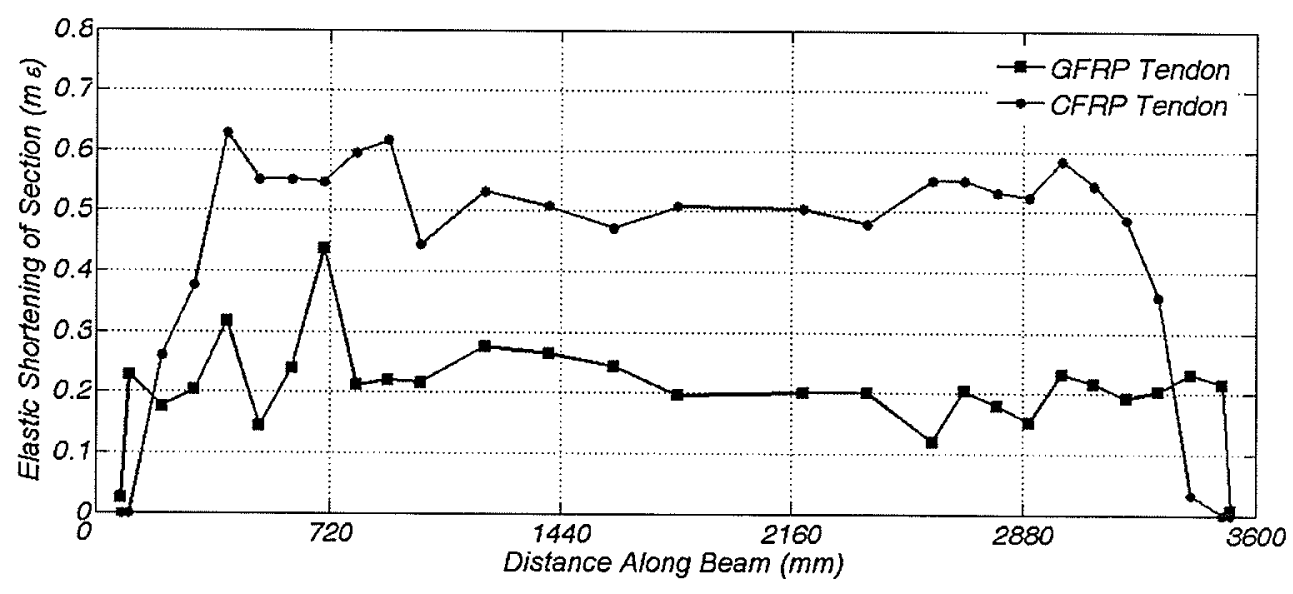

(b) Elastic Shortening of Concrete Section - Demec Points

Figure 5.14 - Transfer Length Determination 


\subsubsection{Development Length Tests and Results}

Shortly after release of prestress, the beams were tested in flexure using a $1000 \mathrm{kN}$ capacity MTS testing machine. Supports were welded to steel plates embedded in concrete masonry blocks, which served as a bearing assembly carrying the load to the structural floor.

\subsubsection{Beam Instrumentation}

During each test conducted on the control beams, deflections were monitored with Linear Variable Displacement Transducers (LVDTs) placed at three locations along the length spanning between the supports. An additional LVDT was placed horizontally on that part of the tendon extruding from the end of the beam. This LVDT was used to monitor slippage during the test. A total of three PI gauges were placed at 45,115 , and $175 \mathrm{~mm}$ from the bottom of the beam at two locations along its length to record strain profiles at different loading stages of the test. More specifically, PI gauges at the reinforcing level $(45 \mathrm{~mm})$ were used to compare strains in the concrete to those in the tendon.

Strain gauges bonded to the surface of FRP tendons chosen for this research were used to record strains during the test and establish development length. All deflection, strain and load readings were recorded using a 64-channel DAQ system.

\subsubsection{Flexural Bond Length}

For beams such as those tested in this research, appearance of the first crack occurs under the point of load and maximum moment. As the crack initiates, tendon stresses at this location will amplify noticeably. As the applied load increases, additional cracks will develop adjacent to the point of load. Due to the tensile resistance of intact concrete, stresses in the tendon will dissipate between cracks and peak at or near the location of a crack. These resulting stress variations are 
proportional to those of strain shown in Figures 5.15 and 5.16. These strain readings were recorded during each development length test using the 37 strain gauges placed on the surface of each tendon in the longitudinal direction. Corresponding to the condition at ultimate immediately before rupture of the bar, the strain readings were used to determine the flexural bond length of each tendon.

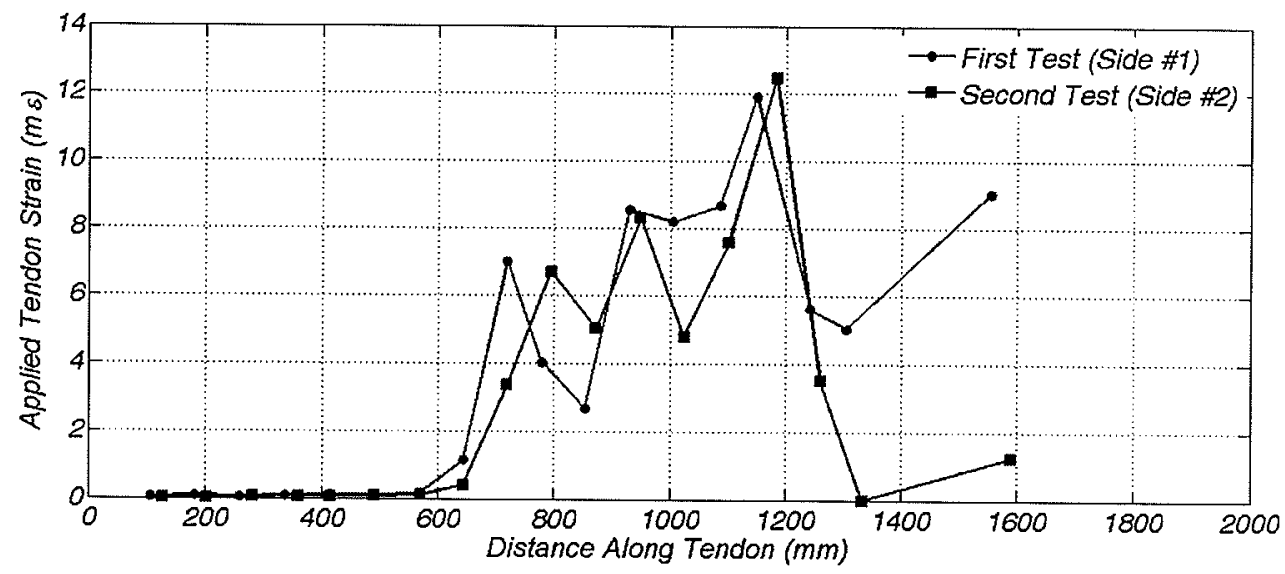

Figure 5.15 - Strain Variation at Ultimate for GFRP Prestressed Beam

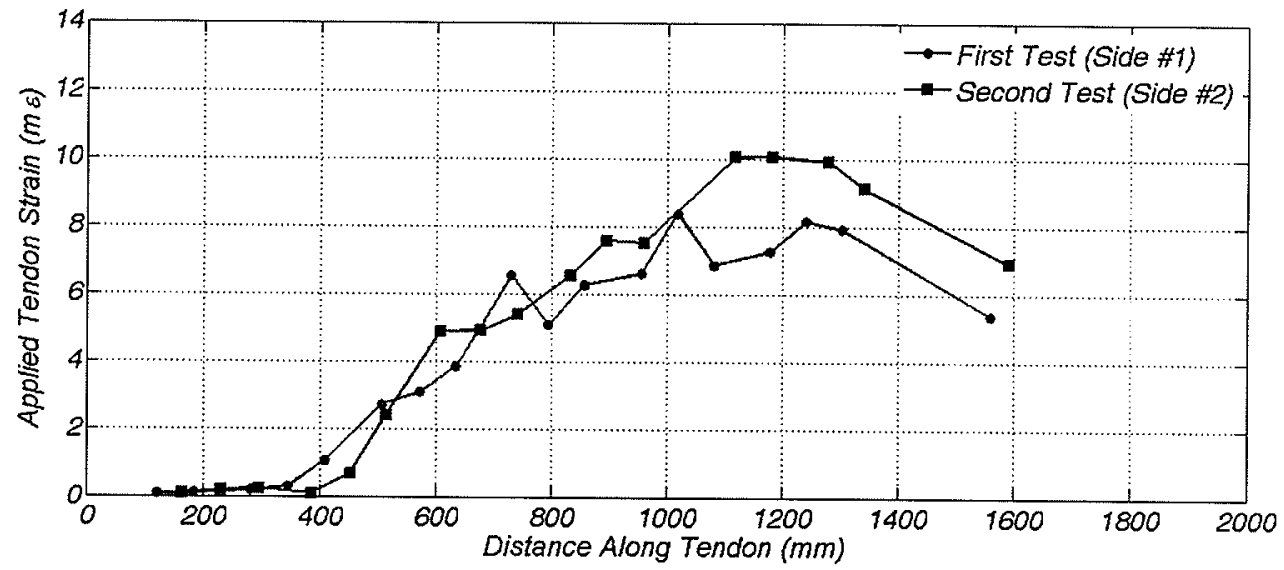

Figure 5.16 - Strain Variation at Ultimate for CFRP Prestressed Beam

As previously stated, the flexural bond length is defined as the shortest embedment length that will cause rupture of the tendon in a prestressed concrete element. As illustrated by force equilibrium in Figure 5.17, tendon stresses will increase from the effective prestressing stress $f_{p e}$ 
to the ultimate strength $f_{p u}$ within this length. This is equivalent to the condition where tendon stresses increase from zero to that applied during a test when rupture occurs. The flexural bond length can therefore be obtained from Figures 5.15 and 5.16 for each tendon by measuring the length between zero strain and maximum applied strain during the test when rupture occurs. On the basis of strain readings recorded during individual tests, the flexural bond lengths were determined to be $597 \mathrm{~mm}$ for the GFRP tendons and $669 \mathrm{~mm}$ for the CFRP tendons.

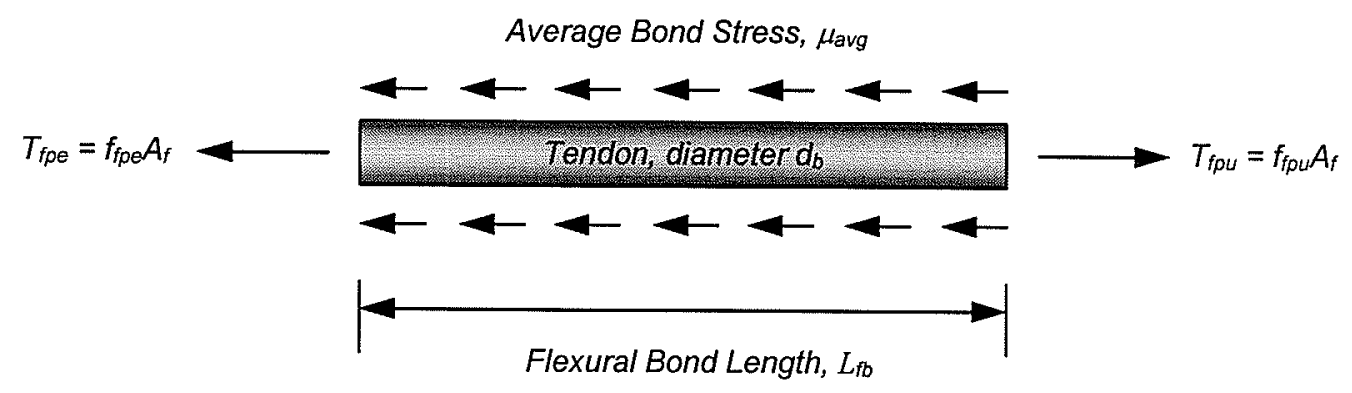

Figure 5.17 - Bond and Tendon Stress Relationship Within Flexural Bond Length

\subsubsection{Development Length and Average Bond Stress}

The development length can now be determined and written as the sum of the transfer and flexural bond lengths that have been determined experimentally for each tendon. In light of previously reported values, the development length was found to range from $740 \mathrm{~mm}$ for the GFRP tendon to $985 \mathrm{~mm}$ for the CFRP tendon. These development lengths are confirmed in Figure 5.18 for each tendon investigated.

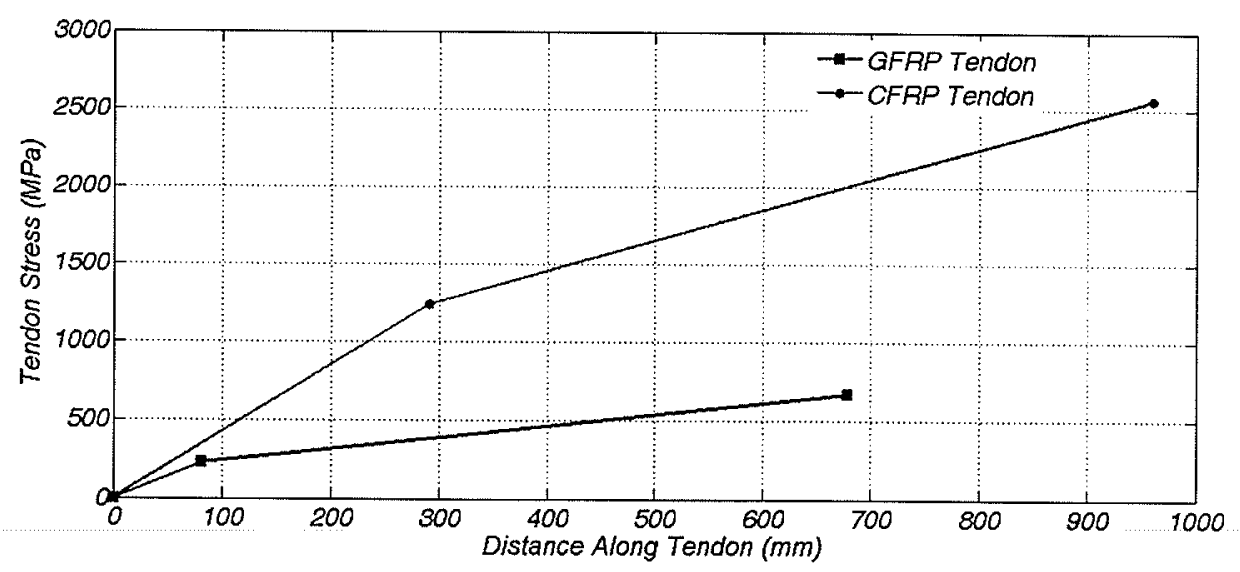

Figure 5.18 - Development Length of Tendons 
Moreover, the average bond stress in the transfer and flexural bond regions of prestressed elements can be determined for both tendons used in this research. These bond stress values are useful in determining the development length for a wide range of prestressing levels and applications involving the use of reinforcement similar to that used in the current project. In reference to surface stresses acting on the tendon shown in Figure 5.17, an equation for the average bond stress can be derived owing to the requirement of force equilibrium.

$\mu_{a v g, f b}=\frac{f_{f p u}-f_{f p e}}{4 L_{f b}} d_{b}$

The preceding equation defines the average bond stress within the flexural bond length. The average bond stress within the transfer length is determined by modifying the equation for tendon stresses that vary between zero and the effective prestressing level.

$\mu_{\text {avg, } t}=\frac{f_{\text {jpe }}}{4 L_{t}} d_{b}$

EQ 5.8

Table 5.5 lists the transfer, flexural bond and development lengths for each tendon while Table 5.6 lists the average bond stresses acting along the length of each tendon. From these results, the CFRP tendon appears to have superior bond performance with bond stress values ranging between 5.1 and $7.5 \mathrm{MPa}$ as opposed to 1.8 and $2.8 \mathrm{MPa}$ for its GFRP counterpart. This is clarified by noticing that the development length for the GFRP tendon is only $25 \%$ shorter than that of the CFRP tendon, which has approximately four times higher tensile strength.

Table 5.5 - Development Length Results

\begin{tabular}{|c|c|c|c|}
\hline Bar Designation & $\begin{array}{l}\text { Transfer ength } \\
\text { (m) }\end{array}$ & 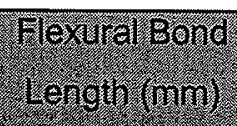 & $\begin{array}{l}\text { Developrient } \\
\text { (ength }(n m)\end{array}$ \\
\hline Aslan 100, GFRP & 143 & 597 & 740 \\
\hline Aslan 200, CFRP & 316 & 669 & 985 \\
\hline
\end{tabular}


Table 5.6 - Average Bond Stress

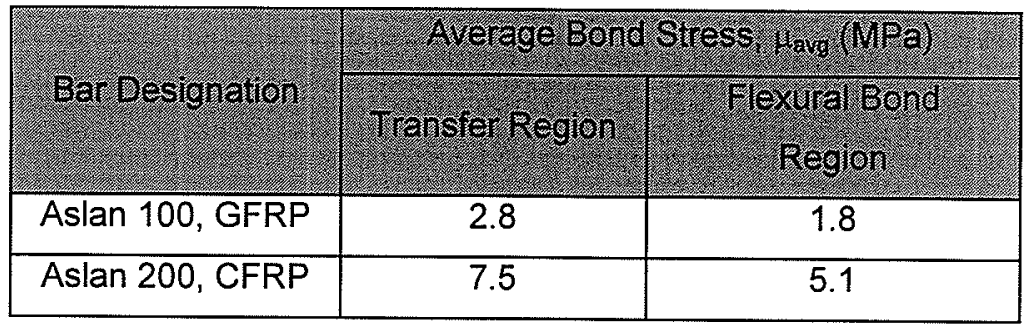

\subsubsection{Crack Formation and Pattern}

The progression of a crack induced by flexural tensile stresses is affected by many parameters. The most relevant include the effective area of concrete in tension, the number of bars, the reinforcing cover, the strain gradient from the level of reinforcement to the tensile face and the strain in the flexural reinforcement. Aside from providing minimal concrete cover and a single prestressing tendon, the relatively low elastic modulus of FRP reinforcing material will cause higher strain gradients within the section. Leading to a smaller neutral axis depth than that typically expected in steel reinforced concrete, fewer and wider cracks were expected during the tests. More specifically, closer and narrower cracks are expected in beams prestressed with CFRP tendons when considering the higher modulus of such reinforcing material.

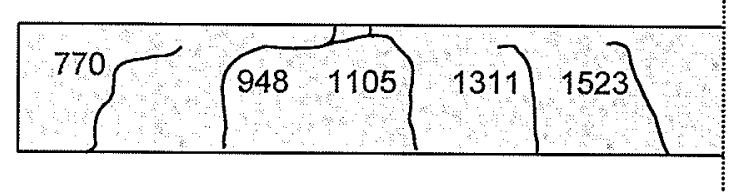

(a) GFRP - First Test

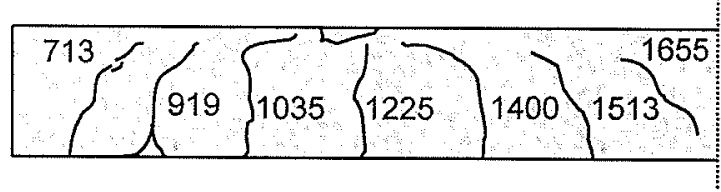

(b) CFRP - First Test

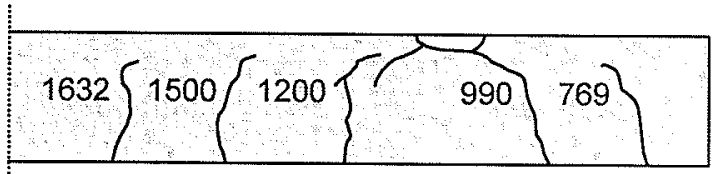

(b) GFRP - Second Test

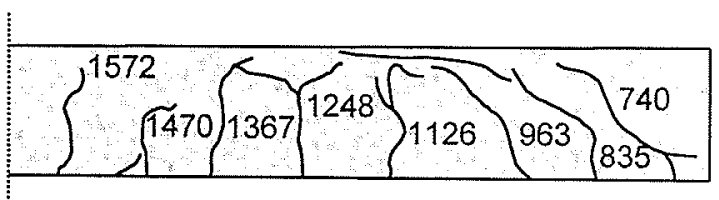

(c) CFRP - Second Test

Figure 5.19 - Crack Pattern and Spacing [mm]

Figure 5.19 shows the crack pattern and location of each crack with respect to the edge of the beam after each test. The figure emphasizes the assertions of the previous discussion by 
revealing a larger amount of closely spaced cracks for the beams prestressed with CFRP tendons. Crack widths were also found to be greater for beams prestressed with GFRP tendons.

\subsubsection{End Slip}

Slip of the tendon at the edge of the beam was monitored during each test. Aside from using an LVDT, slip was also measured on the basis of a reference point placed around the tendon portion extruding from the edge of the beam. The location of this point with respect to the edge of the beam was closely monitored during the tests to ensure reliability of the LVDT readings. Although some readings from the LVDT were recorded during the test conducted on both sides of the GFRP prestressed beam, the existence of slip was not apparent as the location of the reference point did not alter. Departure of readings from both methods was attributed to assembly of the LVDT on the edge of the beam. Although firmly attached to the FRP tendon, the LVDT was only in contact with the surface at the edge of the beam. When cracks form, sudden vibration incurred by the specimens can cause erroneous results characterized by strong fluctuations in slip readings due to unexpected movement of the LVDT tip against the edge of the beam. Such variations in slip readings are clearly shown in Figure 5.20.

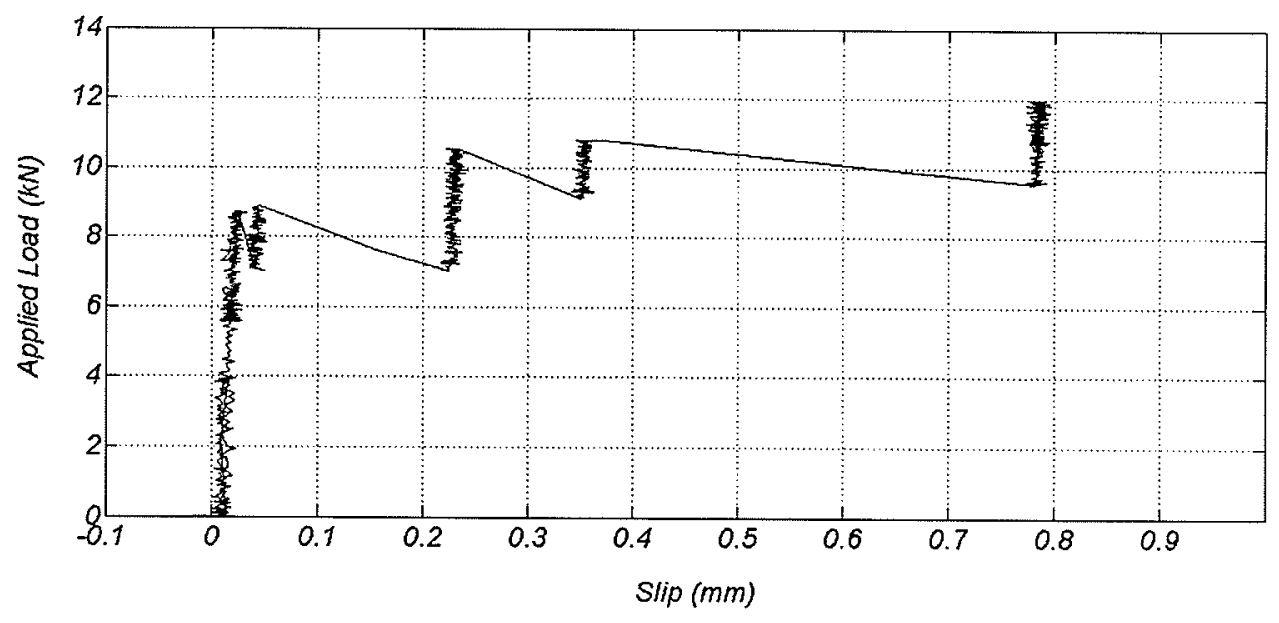

(a) First Test 


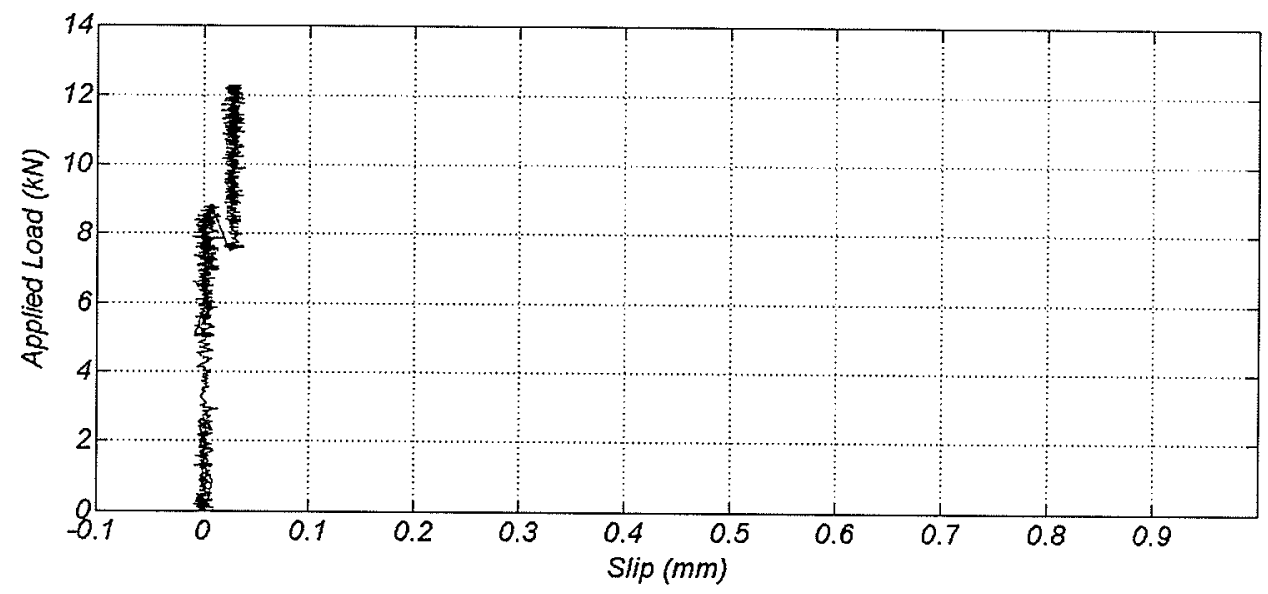

(b) Second Test

Figure 5.20 - Slip Readings - GFRP Tendon

Modifications to the slip monitoring assembly were therefore undertaken for tests conducted on beams prestressed with CFRP, where the LVDT tip was secured to the edge of the beam through the use of a bent screw cemented on the surface. On the basis of these tests, readings from both slip measurement procedures were in agreement. Slip was nonexistent when both sides of the beam were tested, indicating excellent bond of the tendon to concrete. Figures 5.20 and 5.21 illustrate slip measurements obtained from the LVDT during each development length test.

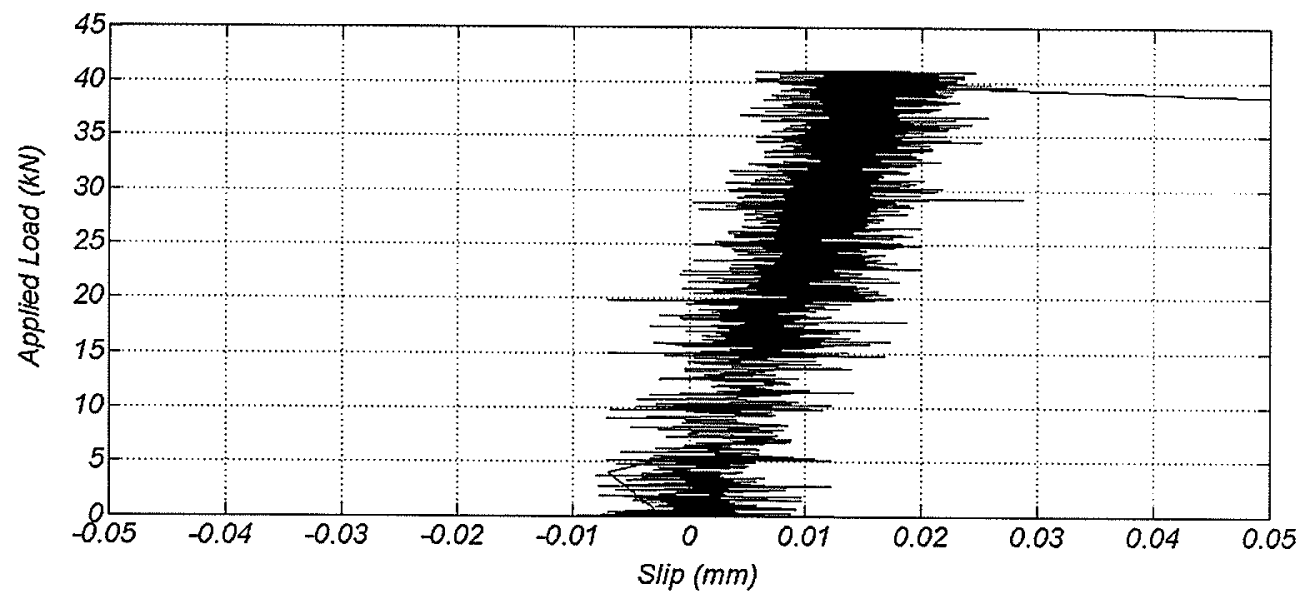

(a) First Test 


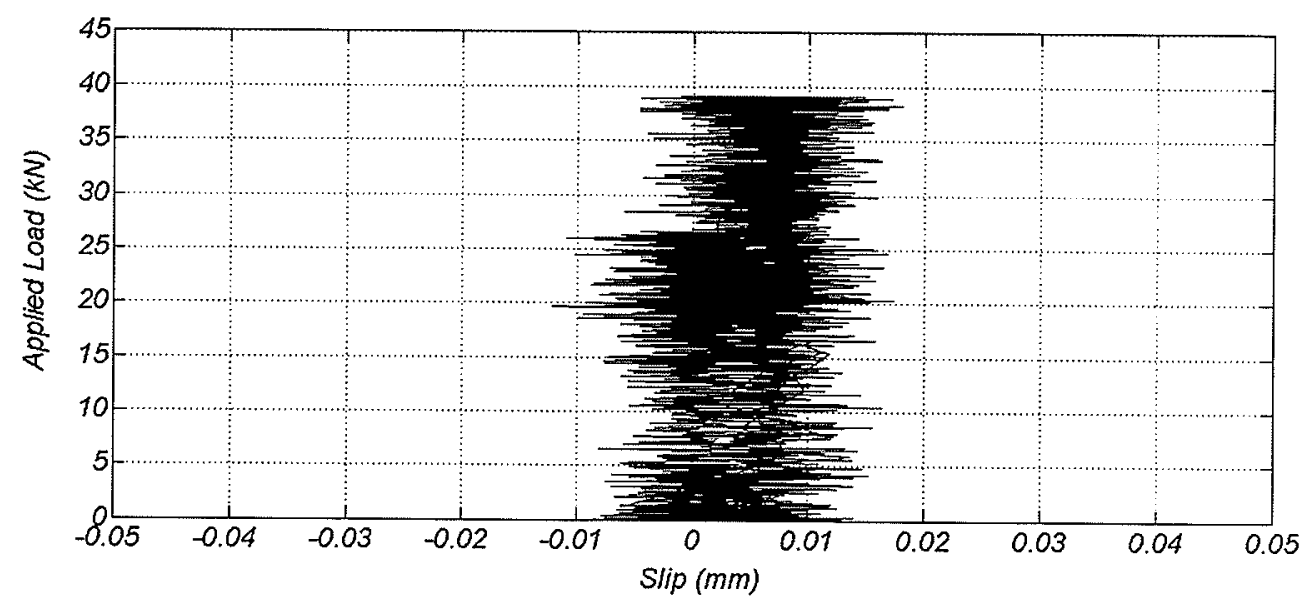

(b) Second Test

Figure 5.21 - Slip Readings - CFRP Tendon

\subsubsection{Moment Curvature}

The moment curvature diagram was plotted for each test to evaluate the flexural behaviour in relation to that expected theoretically. The curvature was obtained on the basis of strain readings recorded from $\mathrm{PI}$ gauges located under the point of load during each test. As indicated in previous sections, these PI gauges were placed at three different locations along the depth of the specimens. As illustrated in Figure 5.22, profiles were generated from strain readings to calculate curvature as defined by EQ 5.8. Lastly, the moment was calculated as the product of the moment arm and applied load.

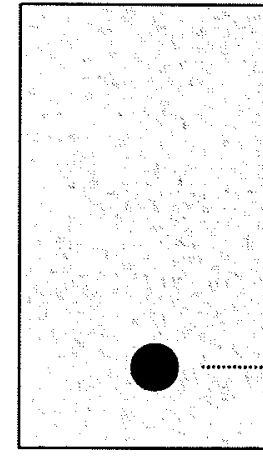

(a) Beam Section

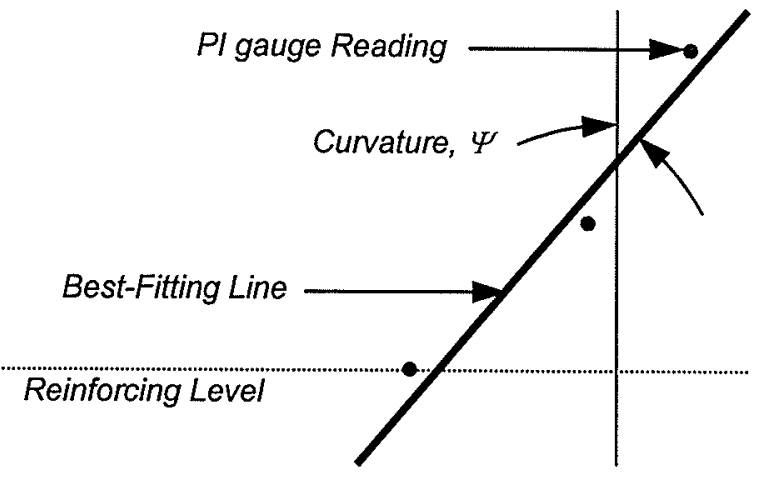

(b) Strain Profile

Figure 5.22 - PI Gauge Readings for Curvature 


$$
\Psi=\frac{\varepsilon_{t o p}+\varepsilon_{b o t}}{h}
$$

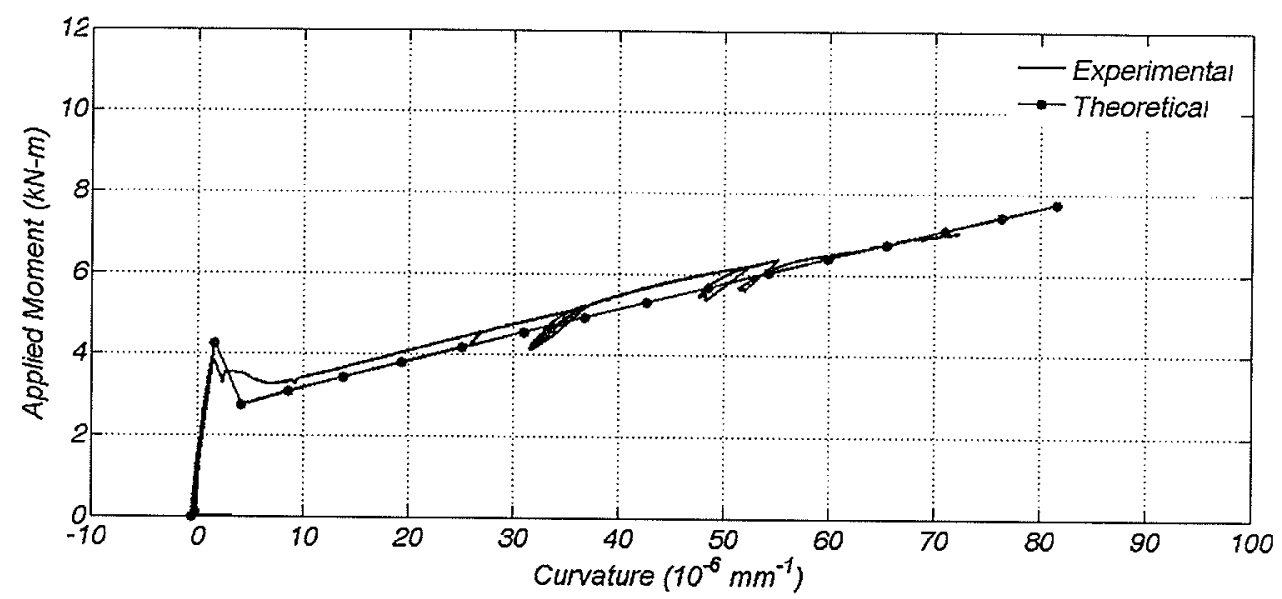

(a) First Test

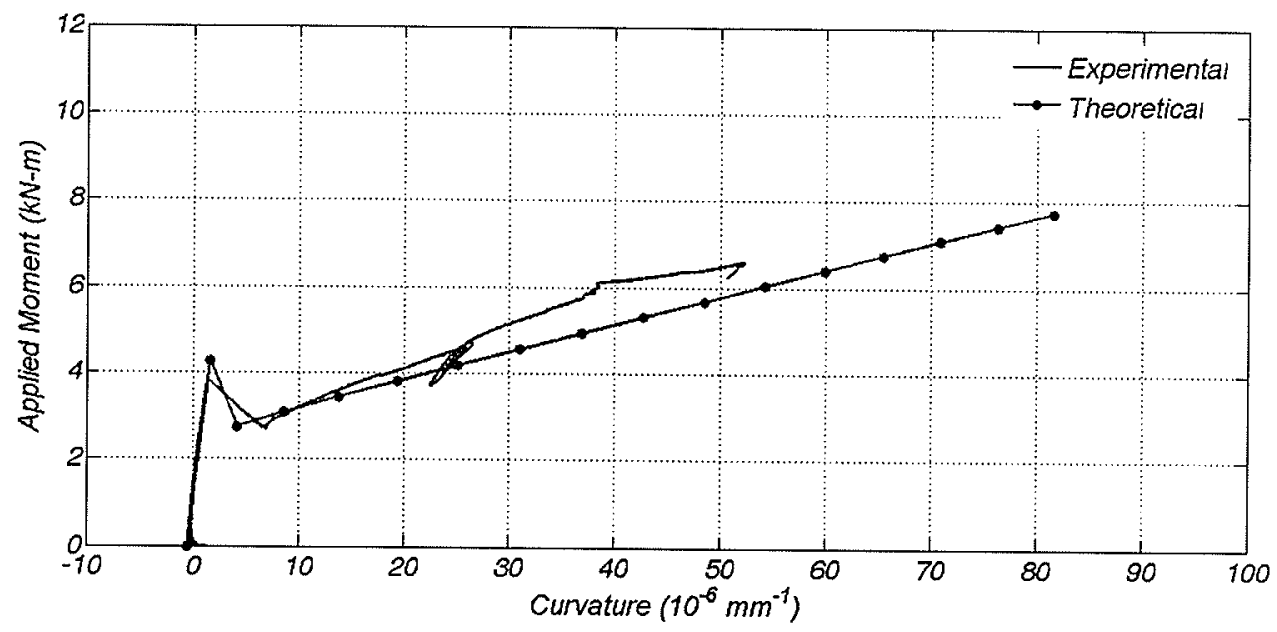

(b) Second Test

Figure 5.23 - Moment-Curvature Diagram - GFRP Tendon

From the moment curvature diagrams shown in Figure 5.23 and 5.24 , it became apparent that experimental results were similar to those described by theory. However, for tests conducted on the second side of each specimen, it also became obvious from experimental results that the stiffness of the beams after cracking was slightly superior than that predicted by theory. This change in stiffness is typical of applications where specimens have lost a fraction of their prestressing force. In spite of bringing supports closer together, the presence of several distinct 
cracks within the span intended for the second test and the possibility of loosing part of the prestress during the first test was unavoidable and led to slight deviations in the flexural behaviour. Nevertheless, these minor digressions were not critical, as strain readings measured on the surface of the tendons did not appreciably differ between tests conducted on the first and second side of each specimen. It ensued that flexural bond lengths resulting from these readings remained unaffected and unvarying.

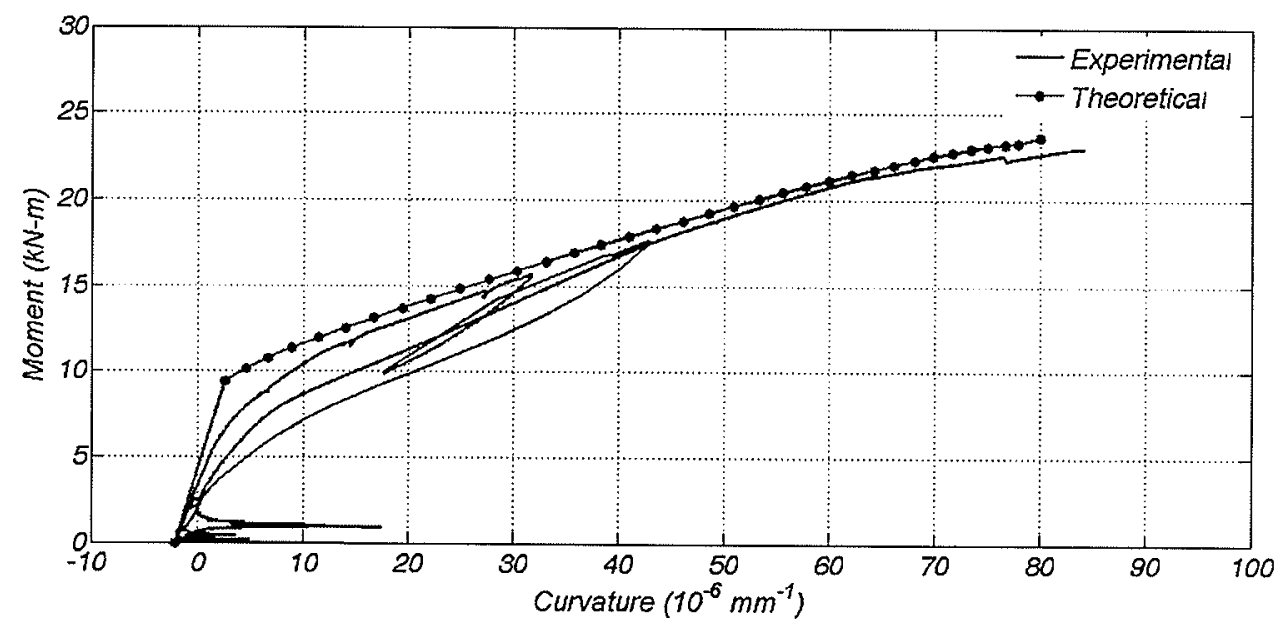

(a) First Test

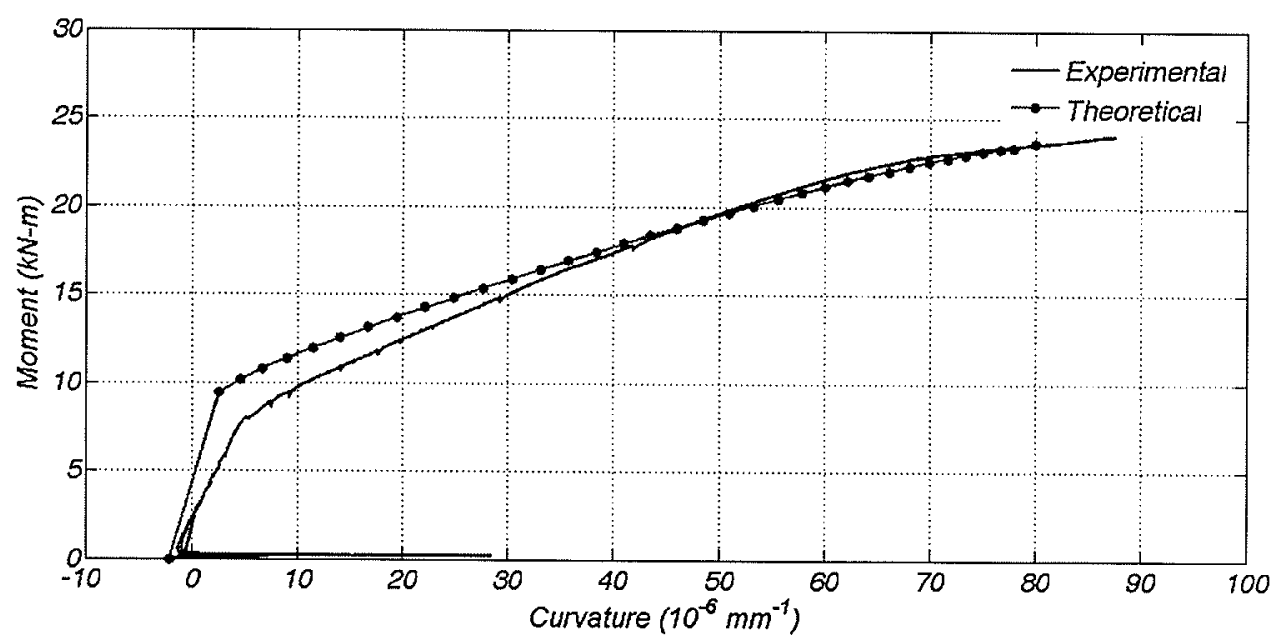

(b) Second Test

Figure 5.24 - Moment-Curvature Diagram - CFRP Tendon 


\subsection{Weathered Beams}

On the basis of results obtained from the two control beams cast at the University of Manitoba, several modifications were brought to the design. In light of these adjustments, an additional 10 beams were fabricated to determine the effect of thermal cycling on bond performance of GFRP and CFRP tendons used in this research. This branch of the experimental program was intended to demonstrate a trend in the load required to cause slippage of the tendon between unweathered and weathered specimens.

\subsubsection{Specimen Modifications}

\subsubsection{Dimensions}

The width and height of the beam intended for weathering were kept at $90 \mathrm{~mm}$ and $200 \mathrm{~mm}$, respectively. The depth of reinforcement was also retained at $155 \mathrm{~mm}$, providing a minimum concrete clear cover of $40 \mathrm{~mm}$ as that required by CSA S806 (2002) for the $9 \mathrm{~mm}$ diameter tendons used in the research. As for the control beams, two $10 \mathrm{M}$ steel reinforcing bars were used as compression reinforcement as well as crack control reinforcement when permissible stresses are exceeded in the tensile zone of the specimens.

\subsubsection{Prestress Level}

At this stage of the project, mechanical properties of the tendons were well known from the results of material testing and behavioural analysis of the two control beams tested. As a consequence, specimens cast for the weathering portion of the experimental program were prestressed to a fraction of the measured tendon ultimate tensile strength. As before, the prestressing level applied to the tendons was selected on the basis of clauses 10.5.1 and 7.1.2.3 
of CSA S806 (2002). Accordingly, CFRP and GFRP tendons were respectively stressed to 60 percent and 30 percent of their ultimate tensile strength.

On the basis of this revised prestress level as well as the losses predicted and measured for the control beams, the initial and effective prestressing stresses were estimated for the weathered beams. These stresses are listed in Table 5.7 and were used to ensure sufficient steel area was provided in the tensile zone of the beams to resist concrete stresses due to the prestressing effect. Using a similar procedure as that used for the control beams, it was concluded that the two $10 \mathrm{M}$ bars placed at the top of the section were sufficient to resist the concrete stresses for a compressive strength of $35 \mathrm{MPa}$ at release and $50 \mathrm{MPa}$ after allowance for all losses.

Table 5.7 - Estimated Tendon Prestressing Level

\begin{tabular}{|c|c|c|}
\hline PRESTRESS LEVEL (MPa) & CFRP (Aslan 200) & GFRP (Aslan 100) \\
\hline Jacking, $f_{f p o}$ & 1,538 & 200 \\
\hline Initial, $f_{f p i}$ & 1,459 & 196 \\
\hline Effective, $f_{i p e}$ & 1,293 & 174 \\
\hline
\end{tabular}

\subsubsection{Testing Procedure}

Single point loading tests were successively conducted on the weathered beams. The load was placed $500 \mathrm{~mm}$ from the support within the development length of each tendon. The load was placed sufficiently far from the support to prevent dowel action and premature failure of the tendon under lateral loads usually expected in short beams. However, proximity of load to the support allowed for bond failures typically characterized by slippage of the reinforcement. In similarity with control specimens, weathered beams were designed to allow tests at each end. Due to the location of load and the ability to maintain the damaged region outside the span intended for the second test, supports were kept at the same location throughout the testing program. The testing procedure is illustrated in Figure 5.25. 


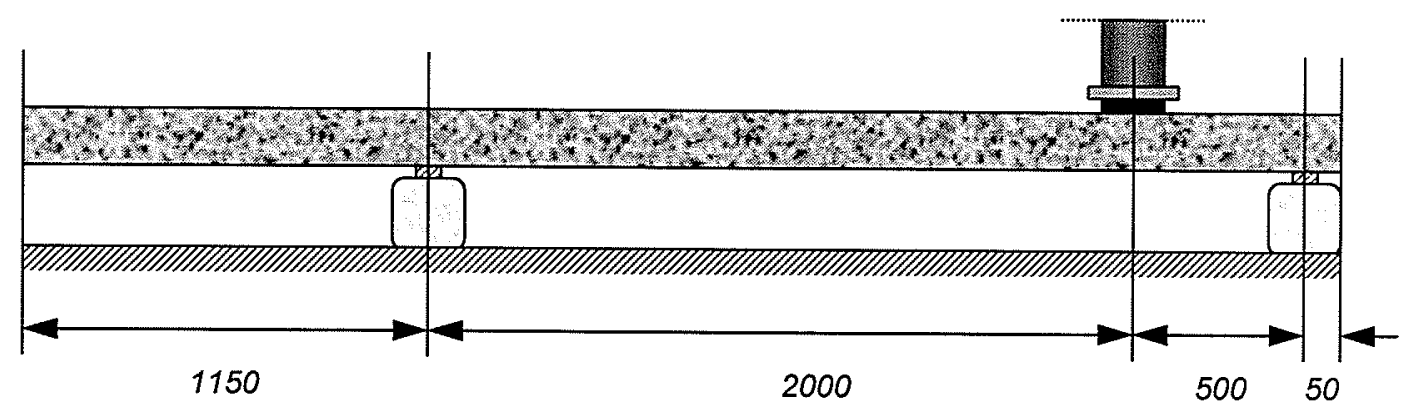

(a) First Flexural Bond Test (Test \#1)

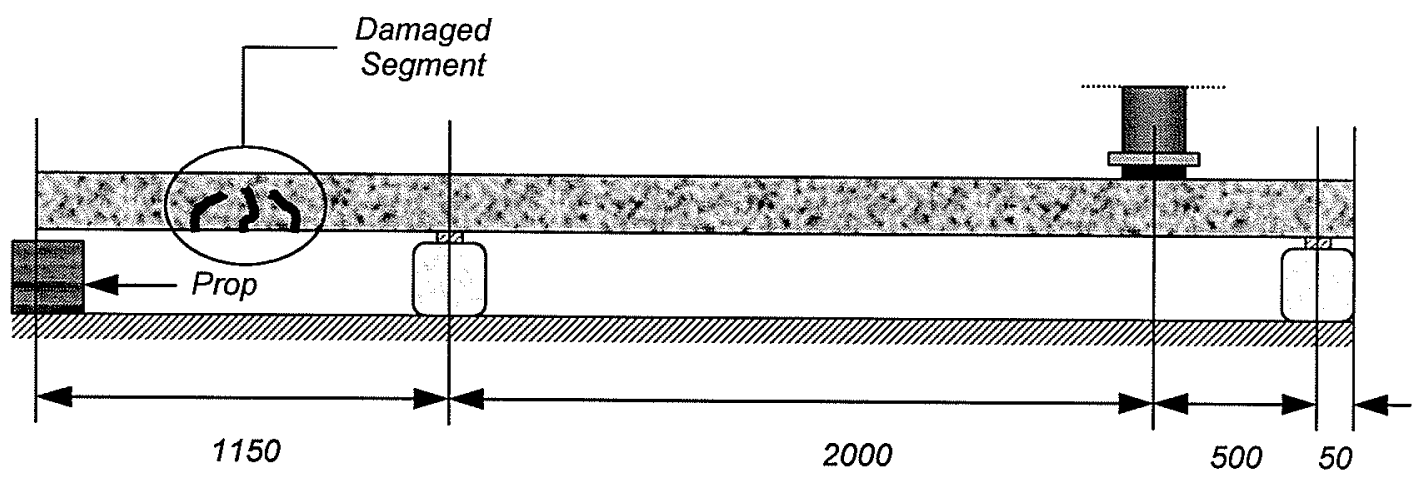

(b) Second Flexural Bond Test (Test \#2)

Figure 5.25 - Testing Procedure

\subsubsection{Shear Reinforcement}

Shear reinforcement was provided along the entire length of the beams, including those prestressed with GFRP tendons. Aside from hindering damage within the portion of the web affecting the second flexural bond test, this reinforcing scheme was adopted to avoid premature failure of the weathered beams near the support where excessive shear is expected.

A total of $37,6 \mathrm{~mm}$ diameter stirrups spaced at $100 \mathrm{~mm}$ on centre were used as shear reinforcement in all beams. The reinforcing scheme is shown in Figure 5.26. 


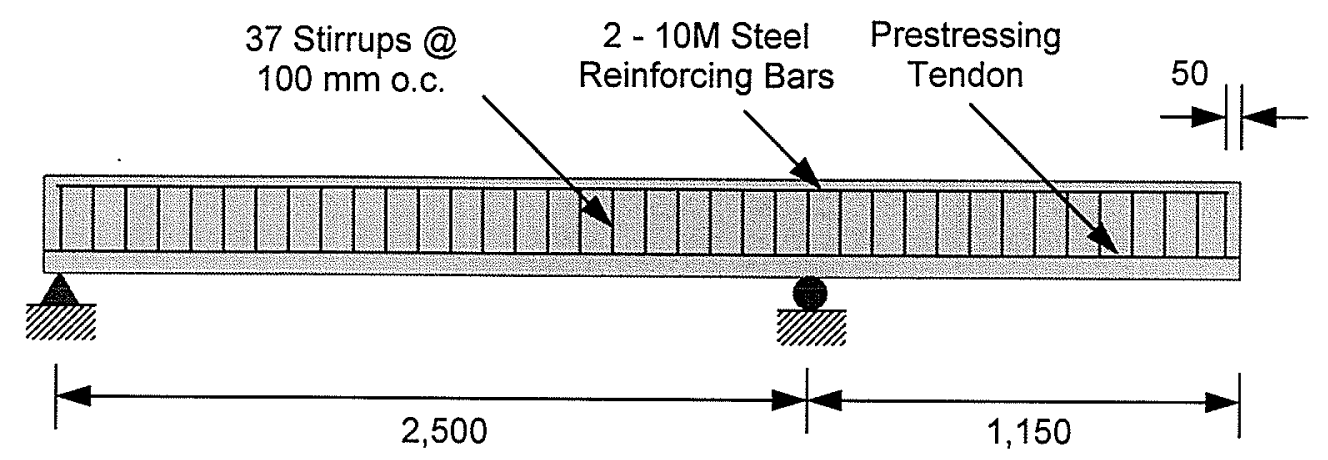

Figure 5.26 - Shear Reinforcing Scheme for Weathered Beams

\subsubsection{Application of Prestress}

Reinforcing cages were fabricated at the University of Manitoba during the month of March 2004. After completion, the cages were shipped to the Precast Plant of LAFARGE Canada for prestressing and casting of the specimens. The prestressing force was applied to the tendons using a hydraulic jack with capacity beyond requirement. Although qualified personnel at the plant regularly calibrate and check accuracy of the hydraulic jack, a load cell was placed at the jacking end to ensure the required prestressing load was achieved. As illustrated in Figure 5.27, an indicator box was used to obtain readings from the load cell.

The prestressing application took place in a total of three beds. These prestressing beds consisted of I shaped steel beams placed on their sides with specimens prestressed and cast within the web section. In the first prestressing bed, a total of four beams were prestressed with CFRP tendons while in each of the remaining beds, a total of three beams were prestressed with GFRP tendons. In light of sustaining the prestressing load, two 1" thick steel bearing plates were used at the jacking end as well as at the dead end. As it was the case for the control beams, sides and bottom of the formwork were assembled around the strand and reinforcing cage before application of prestress. Edges of the formwork were subsequently closed with perforated end plates for casting the same day. 
The prestressing load was applied to the FRP tendons using the same steel coupler mechanism as that used for the control beams. Since the manufacturer could only provide a maximum tendon length of $6 \mathrm{~m}$, an additional coupler mechanism was required to link individual tendons for prestressing several beams within the same bed. Accordingly, anchors within the prestressing bed were threaded and joined with a coupler consisting of two nuts welded at the interface. The coupler mechanism was subjected to tension using a $60 \mathrm{kip}(267 \mathrm{kN})$ capacity testing machine and was able to sustain a load of 45 kips. This load corresponds to twice the value required for the higher prestressing level applied to the CFRP tendons in this portion of the research.

\subsubsection{Hazards Involved in Prestressing Applications}

It was initially intended for this project to prestress a total of 14 beams in four prestressing beds. Unfortunately, the steel strand used to transfer the prestressing force to the first prestressing bed failed during the application procedure. The low relaxation strand had a nominal diameter of 9.53 $\mathrm{mm}\left(3 / 8^{\prime \prime}\right)$ and a capacity of $101.9 \mathrm{kN}$ according to Figure 8.2 .3 of the $\mathrm{CPCl}$ Precast and Prestressed Concrete Design Manual. Due to the relatively high prestressing force required on the CFRP tendons, a number of complications with the prestressing beds and bearing plates occurred during the application of prestress. As a consequence, the steel strand was subjected to several unloading and reloading stages before all complications were resolved. Since the required prestressing level was beyond that recommended in practice and equivalent to 98 percent of the strand's ultimate strength, fatigue failure occurred during the third loading procedure. Strands with a larger nominal diameter of $12.7 \mathrm{~mm}(1 / 2$ ") were subsequently used in the remaining beds. Although no injuries resulted from the prestressing incident, extreme caution is advised in ensuring materials used during prestressing applications have adequate capacity. 

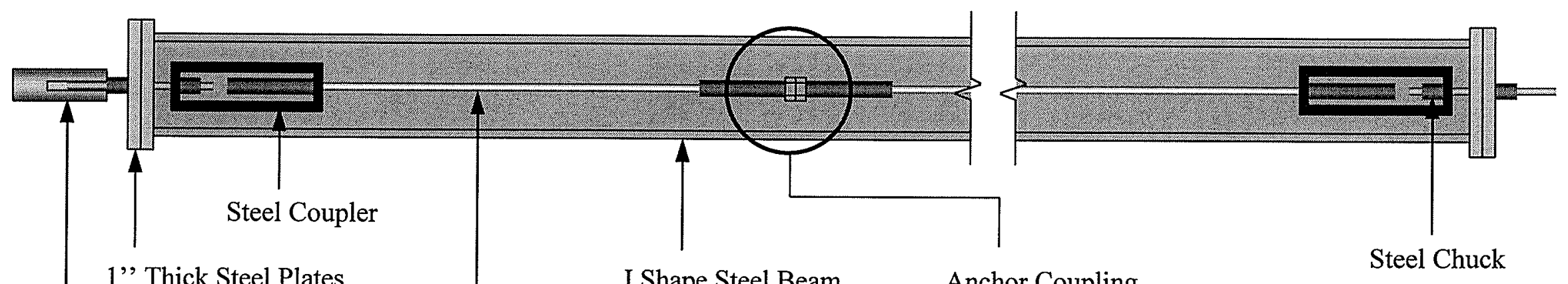

1", Thick Steel Plates

Hydraulic Jack and Indicator Box

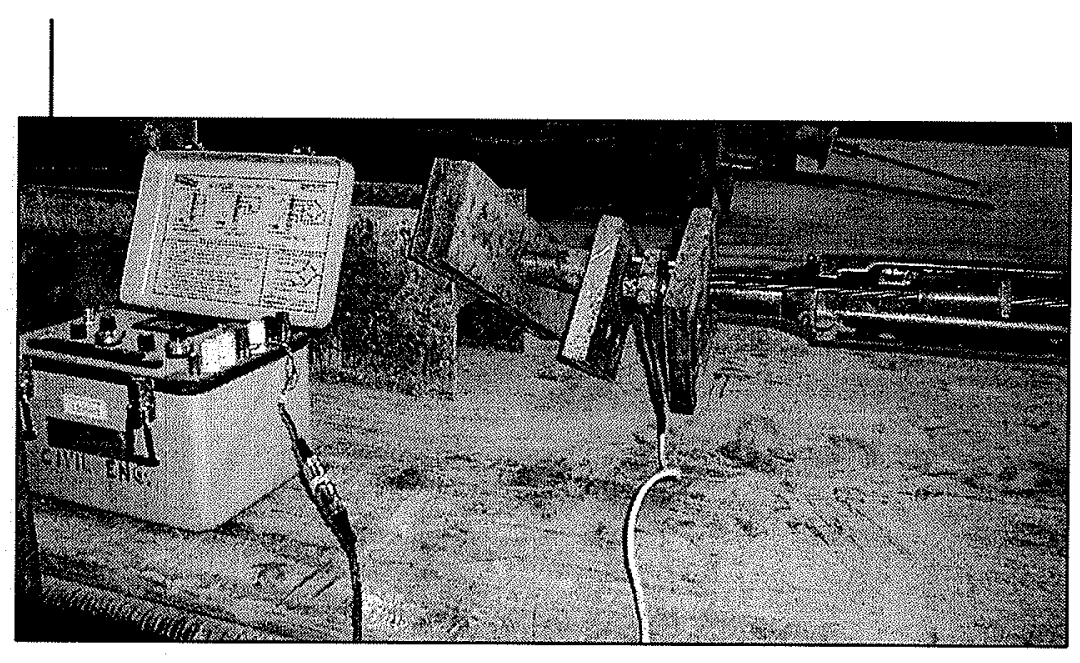

(Prestressing Bed)

FRP Tendon

Anchor Coupling Between Individual Beams

Steel Chuck 


\subsubsection{Prestress Monitoring}

During the application of prestress, strains were closely monitored with two electrical resistance strain gauges in each prestressing bed. A strain indicator box was used to obtain readings from the strain gauges. This procedure is commonly used to verify the prestressing load applied by the hydraulic jack on the basis of elongation. After the application of prestress, strain monitoring was continued until release. Upon release, the strain gauges were detached from the indicator box, allowing beams to be removed from the prestressing bed and handled within the plant.



Figure 5.28 - Release of Prestress

Prestressing was sustained until the required concrete tensile strength was achieved. After a total of 20 days, prestressing was released by cutting steel strands at the jacking end of the prestressing bed with a steel grinder as illustrated in Figure 5.28. Cracks on the top surface of the specimens as well as splitting cracks around the tendons were absent in the transfer zone. The loss of prestress during this period is shown in Figure 5.29. After the release of prestress, an initial loss of 7.4 percent was observed for CFRP tendons while a 5.6 percent loss was observed 
for GFRP tendons. These values are comparable to the ones obtained from the control beams cast in the final stages of 2003. As before, steel strand wires were individually cut to provide gradual and efficient transfer of the prestressing force to the structural elements. Subsequently, tendons were cut at the edge of the anchors to allow extraction of the beams from the prestressing beds. Once extracted, the beams were shipped to the University of Manitoba for future testing.

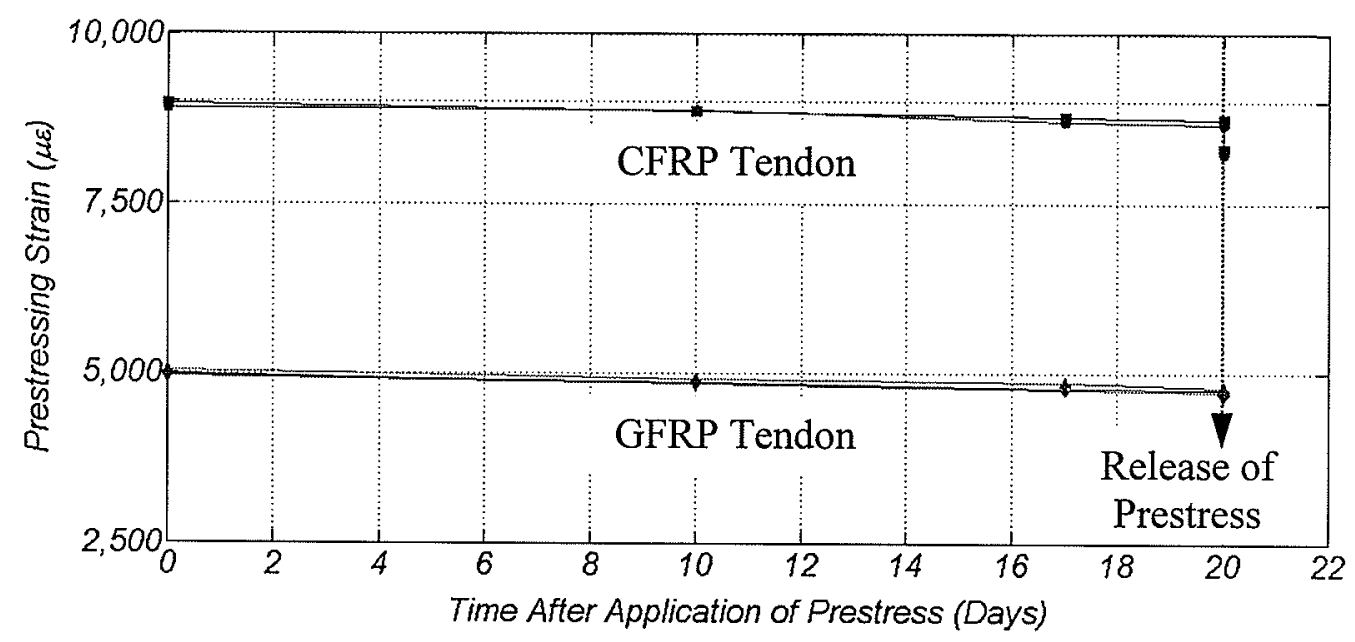

Figure 5.29 - Tendon Strain Variation with Time

\subsubsection{Weathering Process}

\subsubsection{Thermal Cycling}

In the absence of standardized methods for evaluating the bond performance of prestressed FRP tendons in a concrete environment under the effects of service temperatures expected in the Canadian climate, it was initially decided to subject the specimens to temperature cycles ranging between $+40^{\circ} \mathrm{C}$ and $-40^{\circ} \mathrm{C}$. Due to tardiness of the chamber in achieving temperatures of $-40^{\circ} \mathrm{C}$, specimens were initially cycled to a minimum temperature of $-23^{\circ} \mathrm{C}$ as that required by ASTM designation E1512 (2001) for determining the bond strength of adhesive-bonded anchors under the effects of various factors including freezing and thawing action. 
After a total of twenty-five cycles, the sequence was altered such that specimens were subjected to a minimum temperature of $-40^{\circ} \mathrm{C}$ for a total of five cycles. After these additional cycles, thermal weathering was terminated.

Temperature within the beams was monitored by means of a 31-channel DAQ system designed to acquire readings from thermocouples similar to those bonded on the surface of each FRP tendon in this research. Having placed these thermocouples within the transfer regions at the end of each beam, it was possible to compare temperatures recorded near the front of the chamber to those recorded near the back of the chamber where the coil was located to produce thermal gradients.

Strains were also monitored during thermal cycling, on the basis of electrical resistance strain gauges superficially placed on the portion of the tendon extruding from the beams. Readings obtained from these strain gauges were used to determine and confirm the longitudinal and transverse CTEs of the GFRP and CFRP tendons.

\subsubsection{Temperature and Thermal Strain Readings}

Temperature readings from thermocouples located within the beams are shown in Figure 5.30 for cycles ranging between $+40^{\circ} \mathrm{C}$ and $-23^{\circ} \mathrm{C}$ and in Figure 5.31 for cycles ranging from $+40^{\circ} \mathrm{C}$ to $-40^{\circ} \mathrm{C}$. The dotted lines in these figures represent readings obtained from thermal sensors located inside the chamber. These sensors indicate temperature surrounding the beams at any time during the thermal cycles. As illustrated in the figure, extreme temperatures within the chamber were maintained during a total of three hours to achieve equivalent temperatures at the reinforcing level within the beams. Temperature readings from thermocouples were considered sufficiently close to those measured from chamber sensors to confirm aptness of thermal cycles in achieving the desired temperatures within the specimens for the project. 


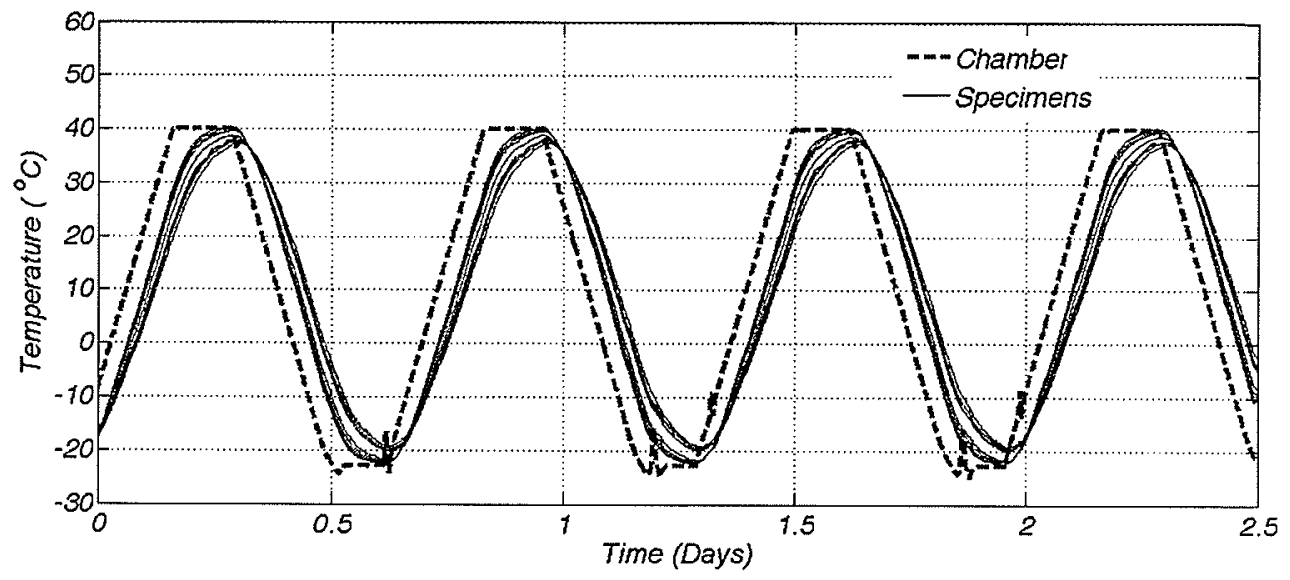

Figure 5.30 - Temperature Readings $\left(+40^{\circ} \mathrm{C}\right.$ to $\left.-23^{\circ} \mathrm{C}\right)$

In Figure 5.32, thermal strain fluctuations with time were plotted as beams were subjected to thermal cycles. As anticipated, the progression of strain under thermal gradients results in a similar trend as that observed for temperature defining thermal cycles within the chamber. It is also shown in this figure, that longitudinal strains (LSG) are less significant than that observed in the transverse direction (TSG). Consequently, longitudinal expansion of the tendon under thermal action is minor, leaving the transverse CTE as the major parameter affecting the development of bursting stresses within the concrete cover.

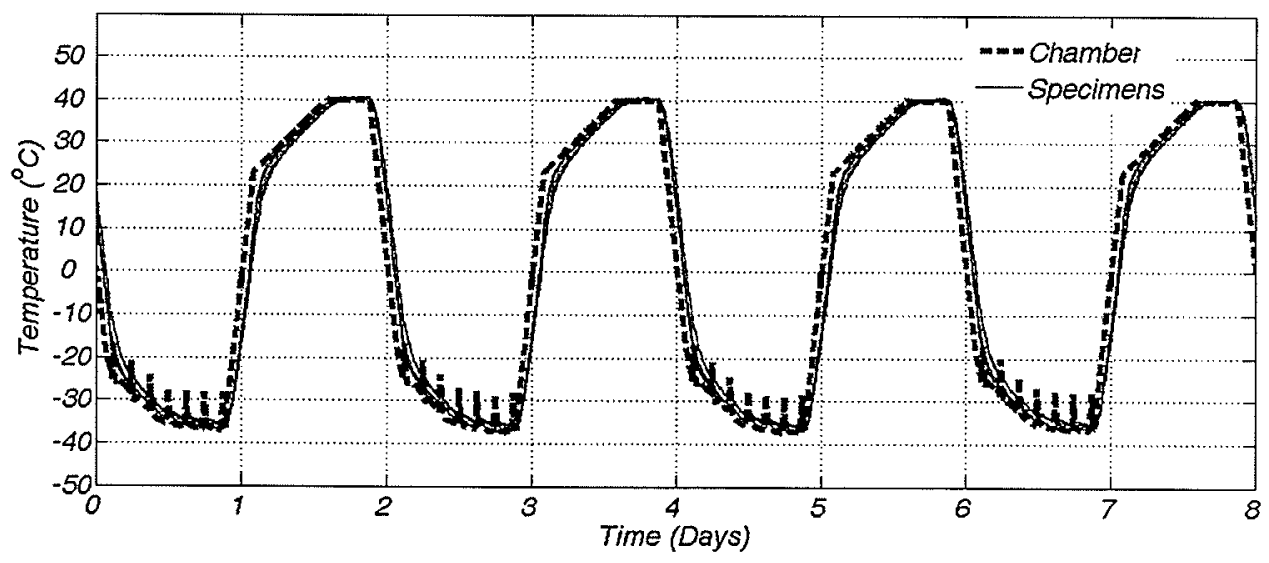

Figure 5.31 - Temperature Readings $\left(+40^{\circ} \mathrm{C}\right.$ to $\left.-40^{\circ} \mathrm{C}\right)$ 


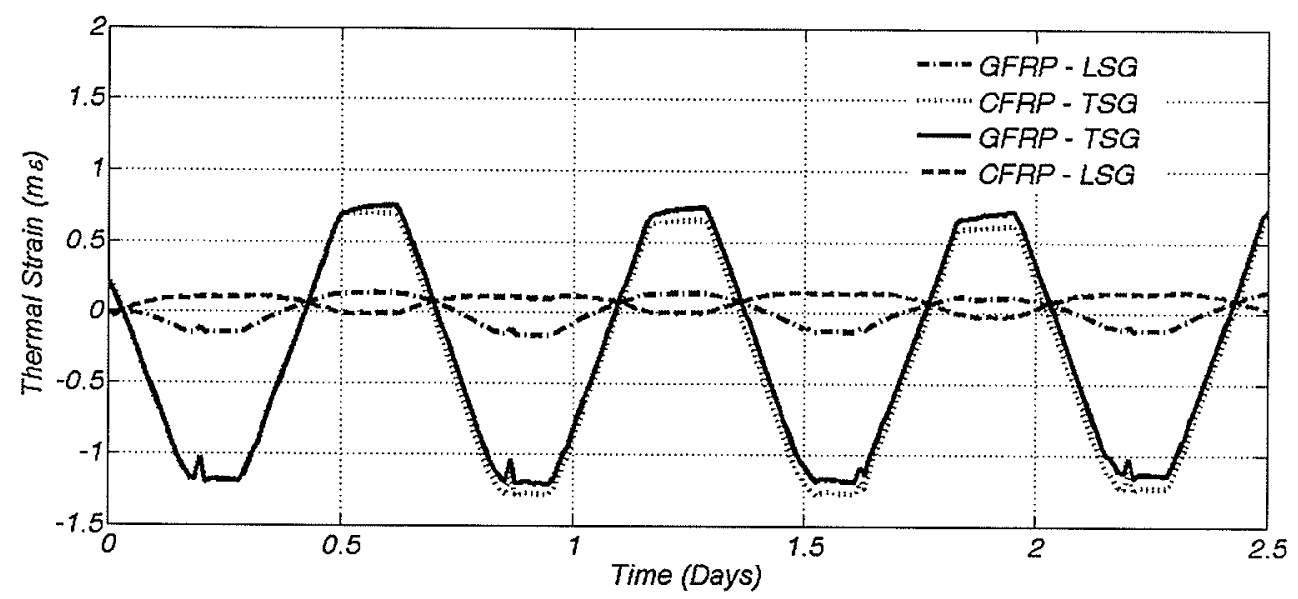

Figure 5.32 - Thermal Strains $\left(+40^{\circ} \mathrm{C}\right.$ to $\left.-23^{\circ} \mathrm{C}\right)$

Figures 5.33 and 5.34 illustrate thermally induced transverse strain fluctuations as a function of temperature within the chamber. Strain readings shown in these figures were obtained from 2 $\mathrm{mm}$ long strain gauges circumferentially mounted on the surface of the tendons. A total of two beams were instrumented with transverse strain gauges. One of these was prestressed with a CFRP tendon while the other was prestressed with a GFRP tendon. It is clear from these figures that the relationship between transverse thermal strain and temperature is linear. Consequently, a linear trend line was fitted to the data with slopes recorded and averaged to give the transverse CTE. Table 2 summarizes the results of this analysis and indicates that the transverse CTE is similar for both tendons although different from that provided by the manufacturer. It is noticeable from this table that the transverse CTE of the GFRP tendon is slightly higher than that listed by the manufacturer while that of the CFRP tendon is much smaller than that provided by the manufacturer. It should also be kept in mind that the value listed for the CFRP tendon is based on typical values from $\mathrm{ACl} 440.1 \mathrm{R}$ (2001), which are generalized for a wide range of commercially available products. Furthermore, the value is for carbon fibres as opposed to composite reinforcement used in the current project. 


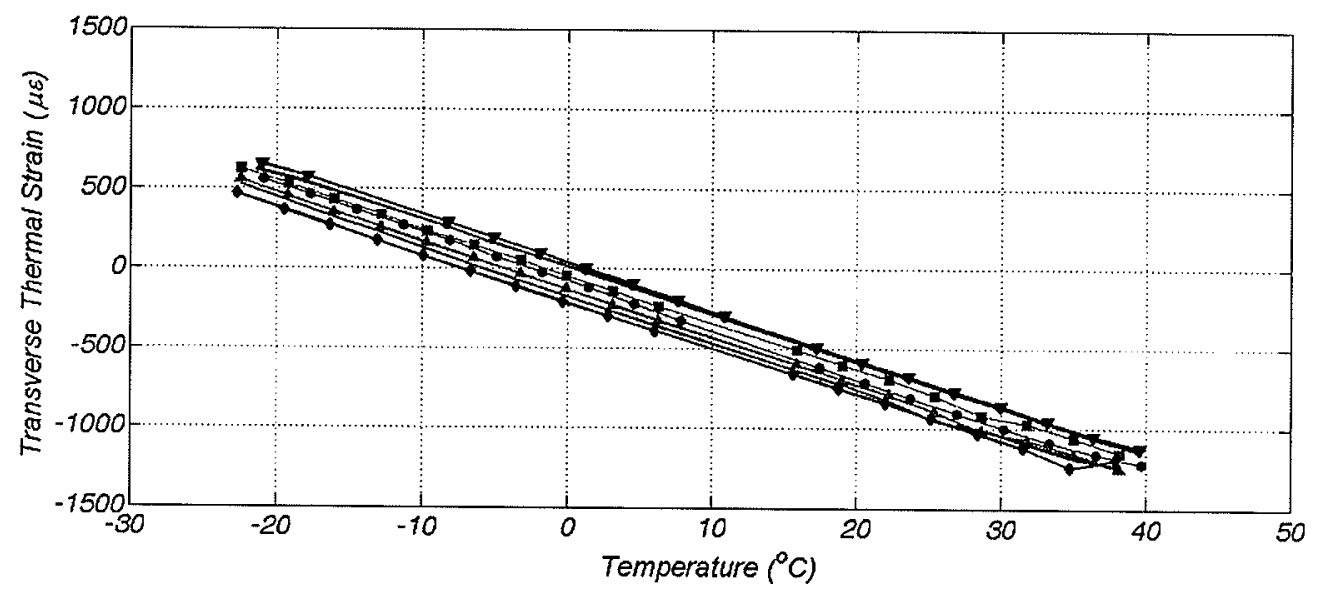

Figure 5.33 - Transverse Thermal Strains (GFRP)

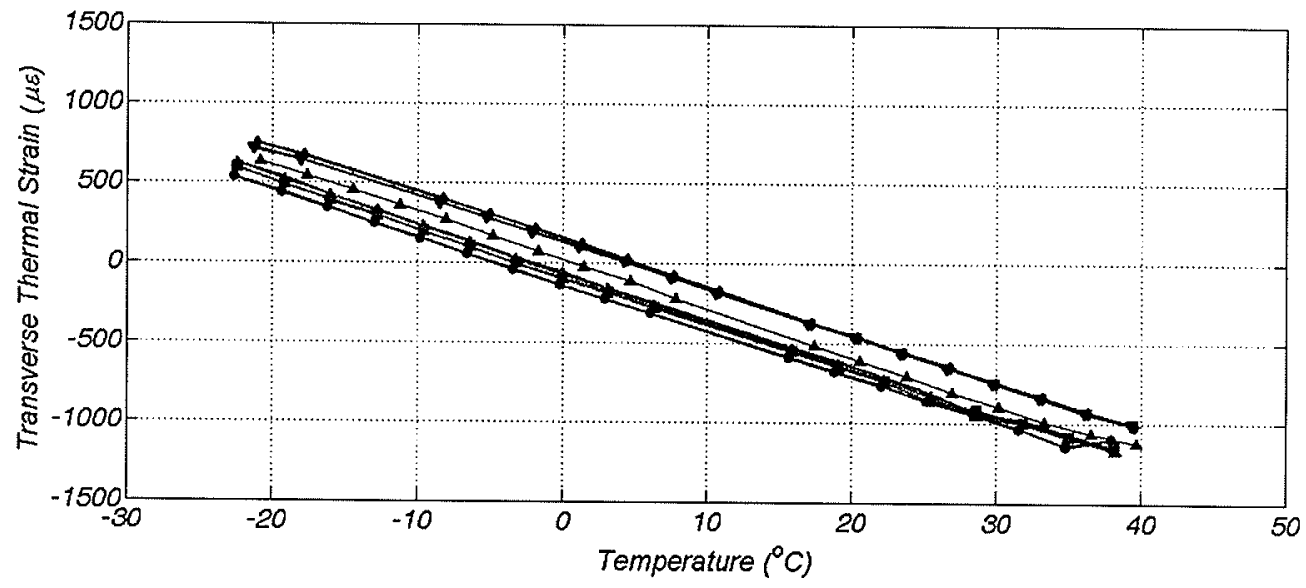

Figure 5.34 - Transverse Thermal Strains (CFRP)

Table 5.8 - Transverse CTE Assessment Summary

\begin{tabular}{|c|c|c|}
\hline Source & Bar Type & Transverse CTE (uel ${ }^{\circ}$ ) \\
\hline \multirow{2}{*}{$\begin{array}{c}\text { Manufacturer } \\
\text { (Hughes Brothers, Inc.) }\end{array}$} & GFRP & 21 to 23 \\
\cline { 2 - 3 } & CFRP & 74 to 104 \\
\hline \multirow{2}{*}{$\begin{array}{c}\text { Thermal Strains } \\
\text { (Experimental Data) }\end{array}$} & GFRP & 29.5 \\
\cline { 2 - 3 } & CFRP & 29.2 \\
\hline
\end{tabular}




\subsubsection{Environmental Chamber}

Thermal cycling was performed using a CONVIRON environmental chamber capable of reaching temperatures varying between $+40^{\circ} \mathrm{C}$ and $-40^{\circ} \mathrm{C}$ without humidity control. Beams were introduced in the chamber at room temperature and placed on several wooden blocks to prevent damage of the floor under excessive bearing stresses. Figure 5.35 shows the staggered layout of the beams inside the chamber. During thermal cycling, this arrangement allowed for efficient airflow within the chamber to achieve the desired temperature inside all beams at the reinforcing level. The arrangement also provided an aisle for individuals to randomly check beams for damage along their length during the weathering process. During these regular checks, beams were superficially inspected at the level of the reinforcement using a $0.1 \mathrm{~mm}$ accurate hand held microscope.

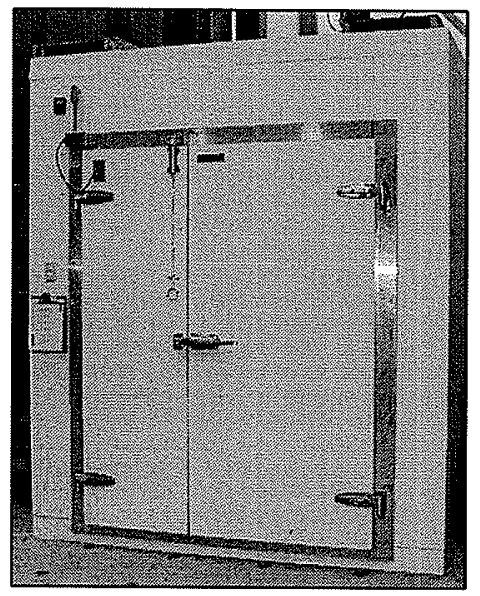

(a) CONVIRON Environmental Chamber

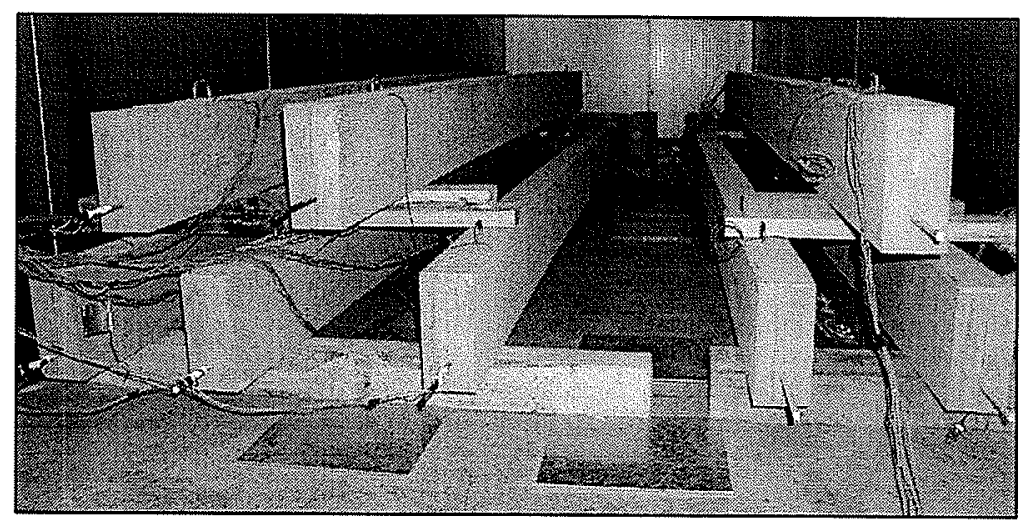

(b) Staggered Layout Inside Chamber

Figure 5.35 - Thermal Cycling Equipment

After thermal cycling, beams were extracted from the environmental chamber to allow preparation of the testing layout. The layout was elaborated to conduct flexural bond tests within the environmental chamber at any temperature ranging between $+40^{\circ} \mathrm{C}$ and $-40^{\circ} \mathrm{C}$. More specifically, half of the specimens weathered and subjected to the thermal cycles were tested at $+40^{\circ} \mathrm{C}$ while the remaining half was tested at $-40^{\circ} \mathrm{C}$. Specimens not subjected to thermal cycles 
were tested at ambient temperature and served as benchmarks to assess the effect of the weathering process.

\subsubsection{Expected Level of Damage}

Based on material properties obtained for beams subjected to the weathering process, critical temperature charts from the linear elastic analysis (LEA) and finite element analysis (FEA) presented in Chapters 3 and 4 of this thesis were used to determine the expected level of damage around the reinforcement. These charts are illustrated in Figure 5.36. The total temperature change accounting for thermal gradients applied to the beams, the prestressing effect and compatibility of axial deformation were calculated to be $169^{\circ} \mathrm{C}$ and $138^{\circ} \mathrm{C}$ for the CFRP and GFRP prestressed beams, respectively. The calculations are based on the procedure described in Chapter 3 of this thesis.

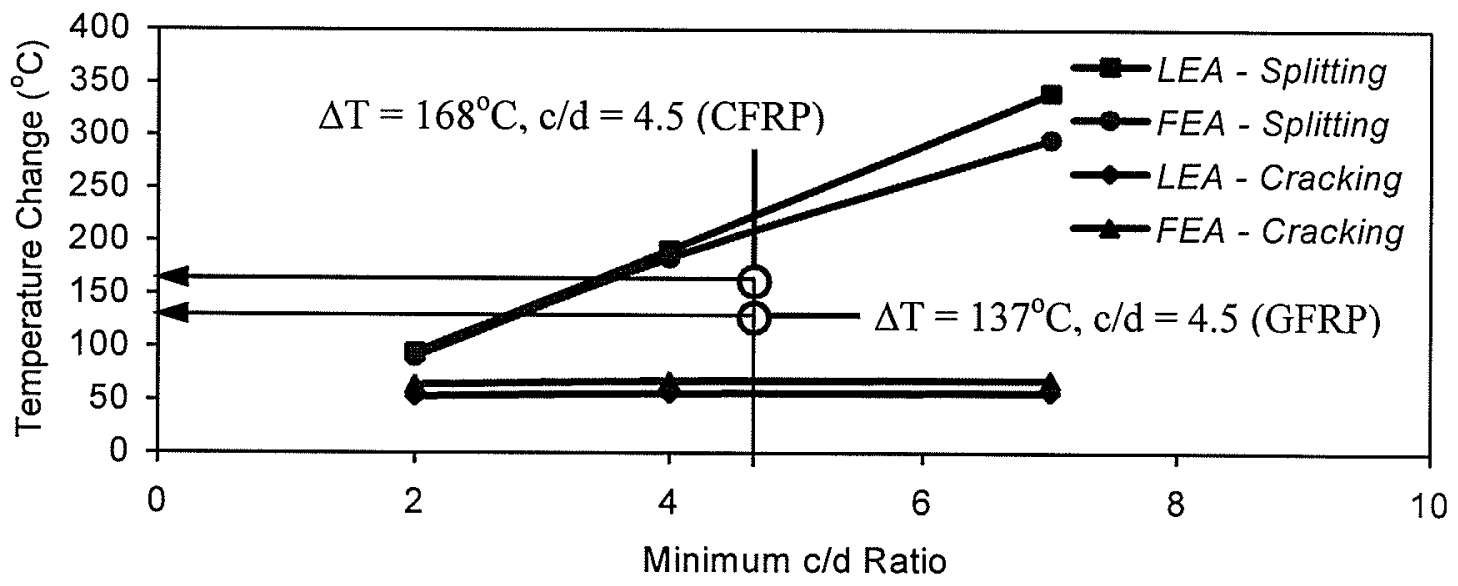

Figure 5.36 - Critical Temperature Chart $\left(\mathrm{f}_{\mathrm{c}}=45 \mathrm{MPa}, \mathrm{E}_{\mathrm{f}}^{\top}=3,000 \mathrm{MPa}, \alpha_{f}^{\top}=30 \mu \varepsilon /^{\circ} \mathrm{C}\right)$

According to Figure 5.36, the total temperature change applied to the beams for this project will not cause splitting of the concrete cover, but will initiate cracking. The extent of damage was monitored during the course of the experimental program with the handheld microscope discussed in the preceding section. As opposed to that predicted from both analyses, cracks were not detected after release of prestress and were still absent after the weathering process. 
Although the little moderating effect of fibres in the transverse direction causes the transverse elastic modulus to be relatively close to that of the polymeric matrix of FRP reinforcement, the lack of standardized methods for determining this value causes those used in the analyses to be questionable. More research should therefore be involved in determining the transverse elastic modulus for FRP reinforcement such as that used in the current research.

\subsubsection{Flexural Bond Tests and Results}

When discussing test results in subsequent sections, beams will be referenced by their names. These names set apart three distinct features. The first part of the name identifies the type of tendon used, $G$ for GFRP and $C$ for CFRP. The second part of the name identifies the temperature level at testing, $\mathrm{M} 40$ for $-40^{\circ} \mathrm{C}, \mathrm{P} 40$ for $+40^{\circ} \mathrm{C}$ and $\mathrm{AMB}$ for ambient temperature. Since each beam allows for two flexural bond tests, the third part of the name identifies the side being tested, $\mathrm{S} 1$ for the first side and $\mathrm{S} 2$ for the second side.

\subsubsection{Testing Layout Assembly}

Due to limited capacity of the chamber flooring to sustain concentrated loads such as that expected from masonry blocks used to support a beam, steel bearing plates were introduced to carry the load directly to the underlying structural floor of the laboratory. As illustrated in Figure 5.37 , each of these plates were supported by four hollow cylinders and bolted to the structural floor using 1" diameter bolts inserted through the chamber flooring. The figure also shows that insulation was provided around the cylinders to prevent thermal leakage through the floor during temperature cycling.

A total of two bearing plates were fixed to the structural floor and overlaid by a five meter long steel beam allowing various simple spans to be tested as the loading point through the chamber ceiling is fixed. A $250 \mathrm{kN}$ capacity MTS actuator was used for loading the flexural specimens 
inside the chamber. The actuator was supported above the chamber by two steel columns anchored to the structural floor. In light of reaching the beams within the weathering environment for testing, a set of extensions was connected to the actuator and infiltrated through a 7" hole perforated through the ceiling of the chamber. Individual extensions were manufactured from hollow structural steel sections (HSS) welded to solid 3" long threaded steel sections at each end. As illustrated in Figure 5.40, extensions were connected through the use of 6" long threaded rods.

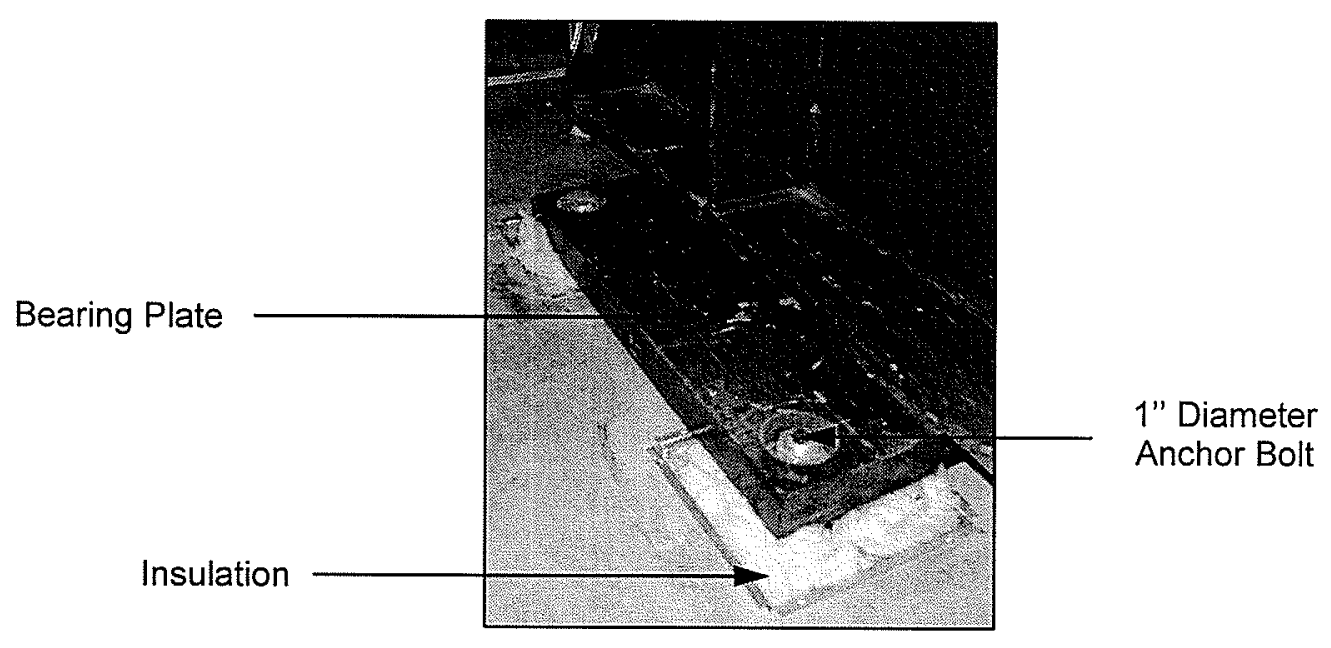

Figure 5.37 - Bearing Plate Assembly

\subsubsection{Beam Instrumentation}

The first beam was introduced and instrumented for testing after implementation of the testing layout within the environmental chamber. Deflections were monitored through the use of four LVDTs placed along the length of the beam. Three of these were placed between the supports while the remaining LVDT was placed at the end of the overhang. In order to monitor slippage during the test, additional LVDTs were placed horizontally on the portion of tendon extruding from each end of the beam. These LVDTs were secured by screws cemented to the surface of the beams. A total of three PI gauges were also placed under the point of load at 45,115 , and 185 
$\mathrm{mm}$ from the bottom of the beam. PI gauges were used to record strain profiles along the depth of the beam at different loading stages of the test.

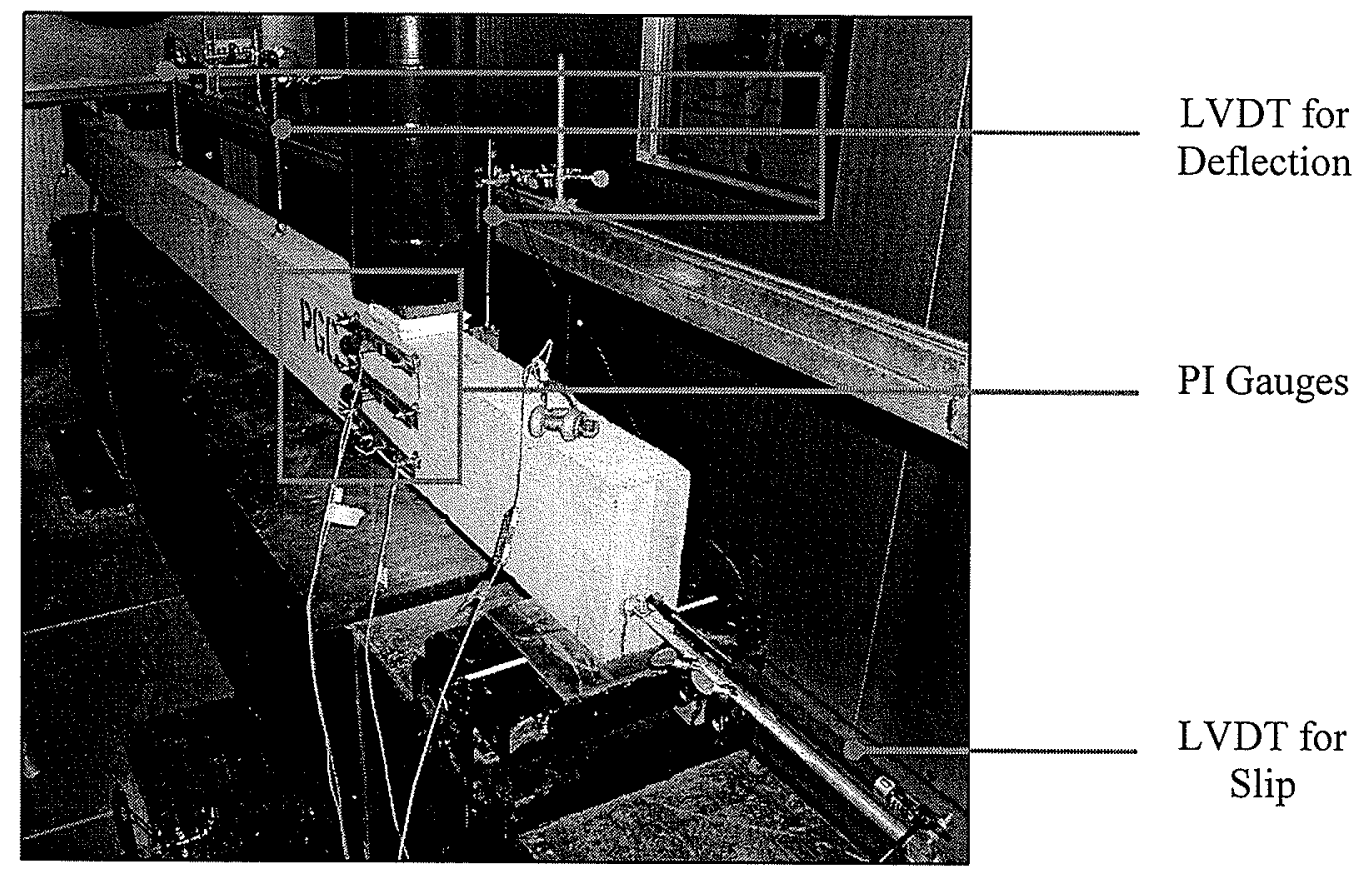

Figure 5.38 - Beam Instrumentation Inside Chamber

Before the application of prestress and casting of the specimens, a total of 10 electrical strain gauges were bonded to the surface of the tendons. These gauges were used to monitor longitudinal strains during flexural tests. Any deviation in strain readings between individual flexural tests would indicate a change in bond interaction between the tendon and concrete. All deflection, strain and load readings were recorded using a 22-channel DAQ system equipped with 8 additional thermal sensor channels for temperature monitor during the tests. Instrumentation of the beam is illustrated in Figure 5.38 with a more detailed illustration of the testing layout in Figure 5.40.

\subsubsection{Strain Readings}

A total of 5 strain gauges were placed at each end of the weathered beams. The first strain gauge was contained within the transfer length of each tendon. If bond is to remain intact at the 
interface between the tendons and concrete, strain readings obtained from this sensor should remain absent as the prestressing force is already being developed within this length. Figure 5.39 illustrates the variation of strain along a GFRP tendon typically observed within the tested span at various loading stages. It is worthy to note that strains within the transfer length of the beams prestressed with GFRP tendons remain unchanged during the test, indicating that the bond interaction between tendon and concrete was preserved in these beams. This was the case for all beams prestressed with GFRP tendons, which includes weathered beams tested at $+40^{\circ} \mathrm{C}$ and $-40^{\circ} \mathrm{C}$ as well as those not exposed to the weathering process and tested at ambient temperature.

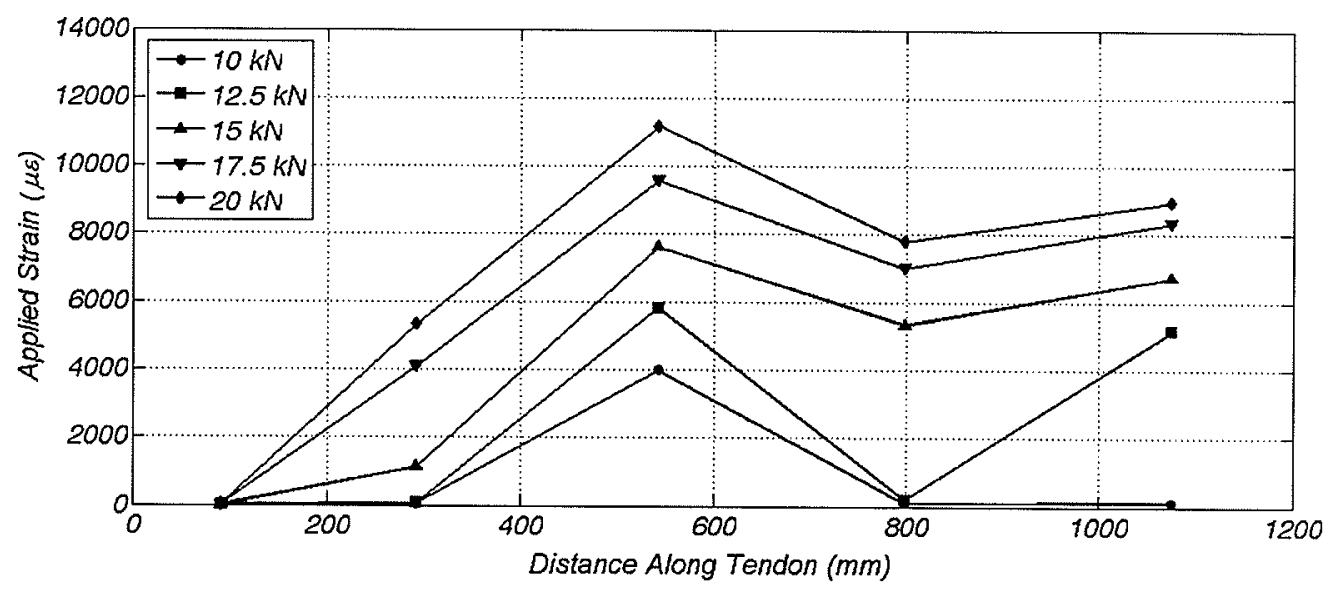

Figure 5.39 - Strain Profile Along GFRP Tendon (G1-P40-S1)

More specifically, bond failures were not observed for any of the beams prestressed with GFRP tendons. In order to confirm whether bond interaction between the materials was affected by the weathering process or testing temperature, the strain variation recorded between strain gauges SG2 and SG3 in Figure 5.41 was closely analysed. This variation in strain was used to determine the change in tendon force between these two locations and measure the ability of the tendon to dissipate flexural tensile stresses within the concrete adjacent to the initial crack formed under the point of load. 


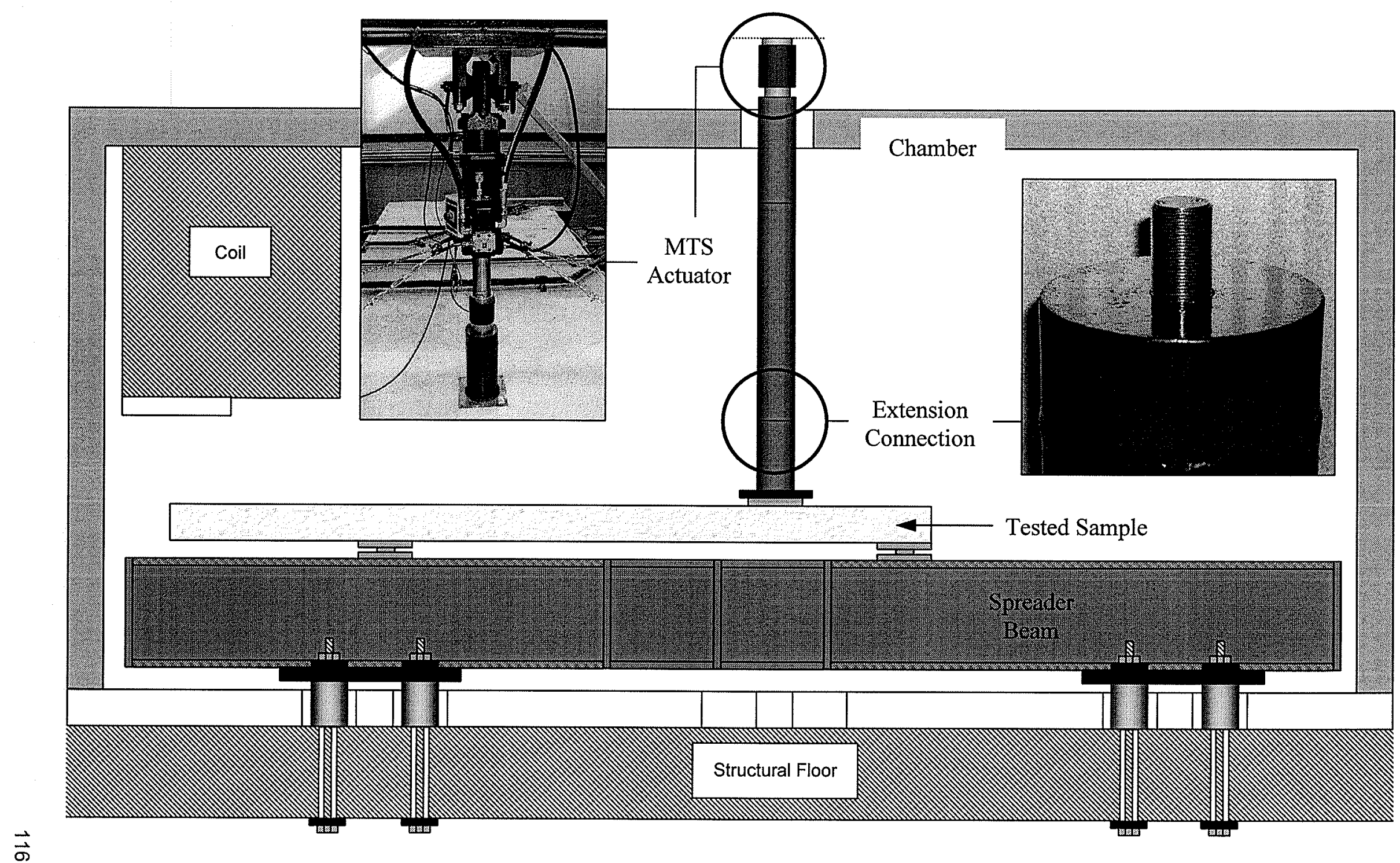

Figure 5.40 - Environmental Chamber Testing Layout 
The tendon force arising from the application of load during the flexural bond tests can be calculated by multiplying strain values obtained experimentally by the modulus of elasticity and cross sectional area of reinforcement. In light of this and in reference to Figure 5.41 , the change in tendon force described by strain gradients recorded between gauges SG2 and SG3 can be calculated at any stage during the tests as the product of this gradient, the modulus of elasticity and cross sectional area of reinforcement.

$$
\Delta T_{f}=\left(\varepsilon_{S G 3}-\varepsilon_{S G 2}\right) E_{f}^{L} A_{f} \quad \text { EQ } 5.9
$$

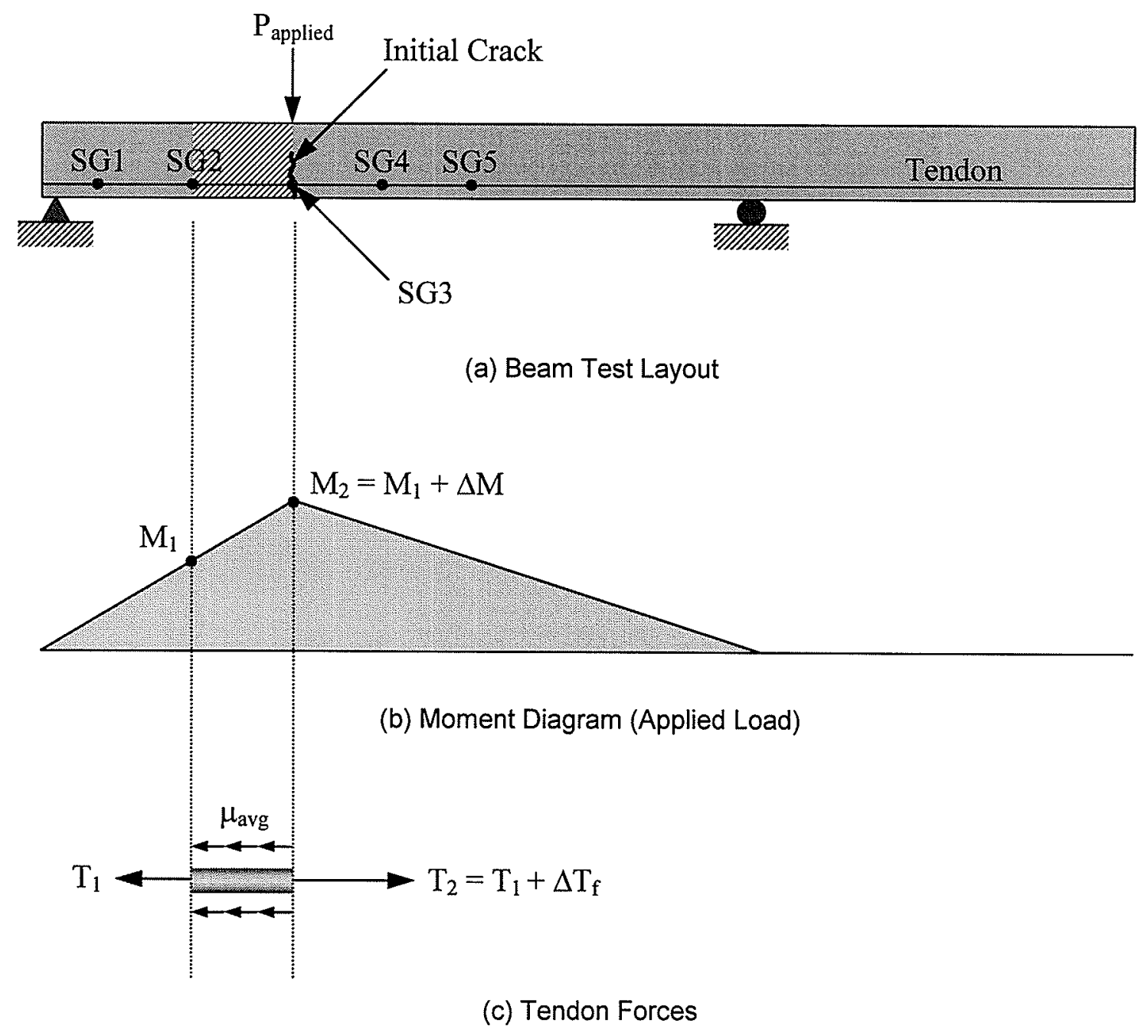

Figure 5.41 - Tendon Force Change $\Delta \mathrm{T}_{\mathrm{f}}$ 
The change in tendon force defined by the previous equation was plotted in Figure 5.42 as a function of the applied load for several of the GFRP prestressed beams tested. Also included in the figure are the results expected from theory acquired from material properties and prestressing levels associated with the current project. Results from the second side of the control specimen (GC-AMB-S2) are not shown in the figure because of deviations in the failure pattern. This side of the beam failed prematurely by rupture of the tendon at a crack formed close to the first support away from the point of load. The failure is shown in Figure 5.43 and is typical for beams with short shear spans as those investigated in this project.

Due to the absence of slip in beams prestressed with GFRP, the load was moved closer to the first support for the second weathered beam tested (G2-P40). For tests conducted on both sides, the load was placed $300 \mathrm{~mm}$ from the first support. Given that slippage remained absent during both tests conducted on this beam, the load was repositioned at $500 \mathrm{~mm}$ from the support for the rest of the experimental program. Due to the position of strain gauges on the surface of the tendon with respect to the initial crack forming under the point of load, results from this beam were not used to examine the change in tendon force between SG2 and SG3. Nevertheless, absence of slippage and tension failure of the beam under the point of load has further demonstrated the excellent bond of the GFRP tendons in concrete despite the weathering process and testing temperature.

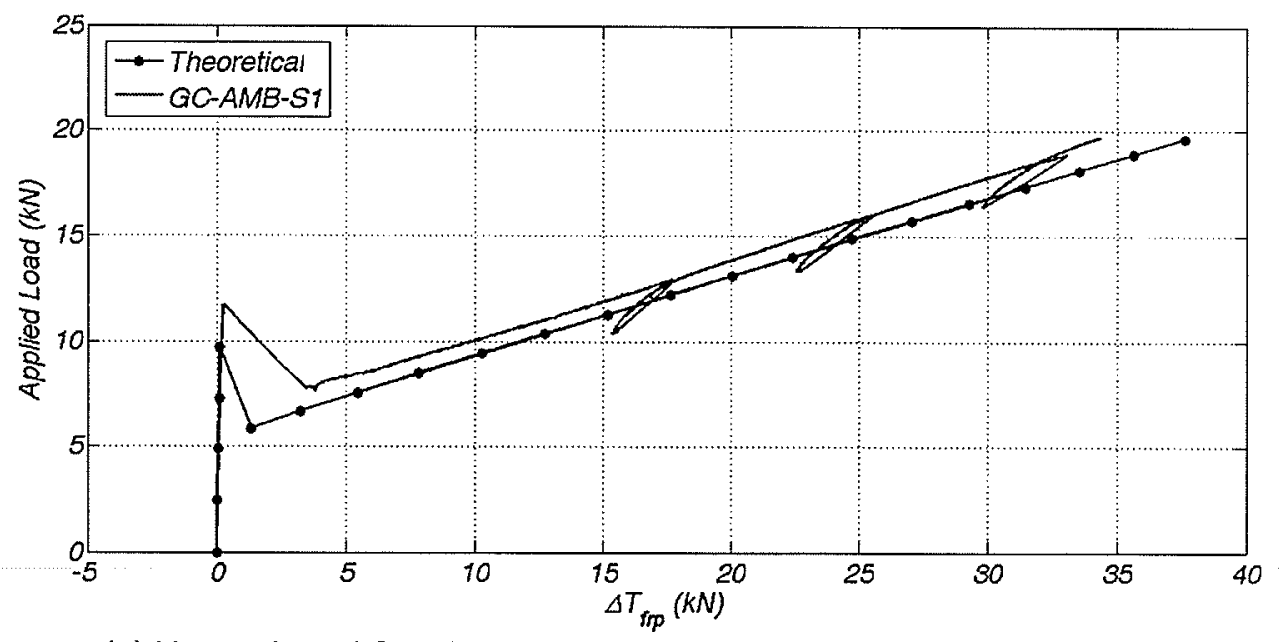

(a) Unweathered Specimen Tested at Ambient Temperature, GC-AMB 


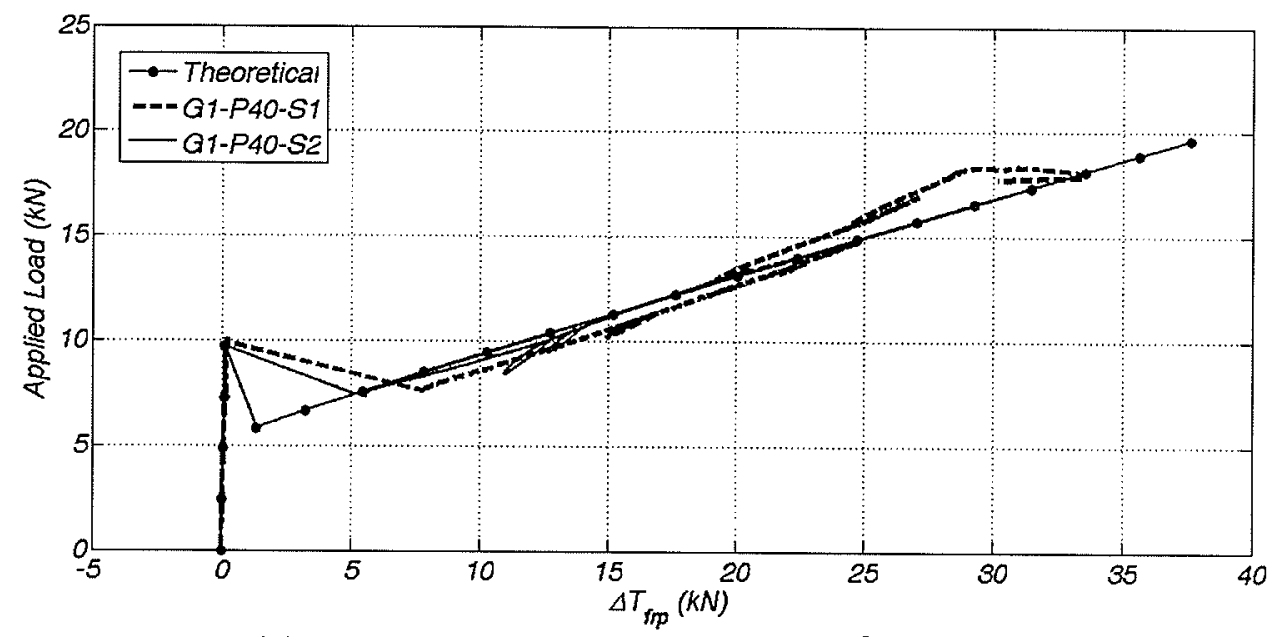

(b) Weathered Specimen Tested at $+40^{\circ} \mathrm{C}, \mathrm{G} 1-\mathrm{P} 40$

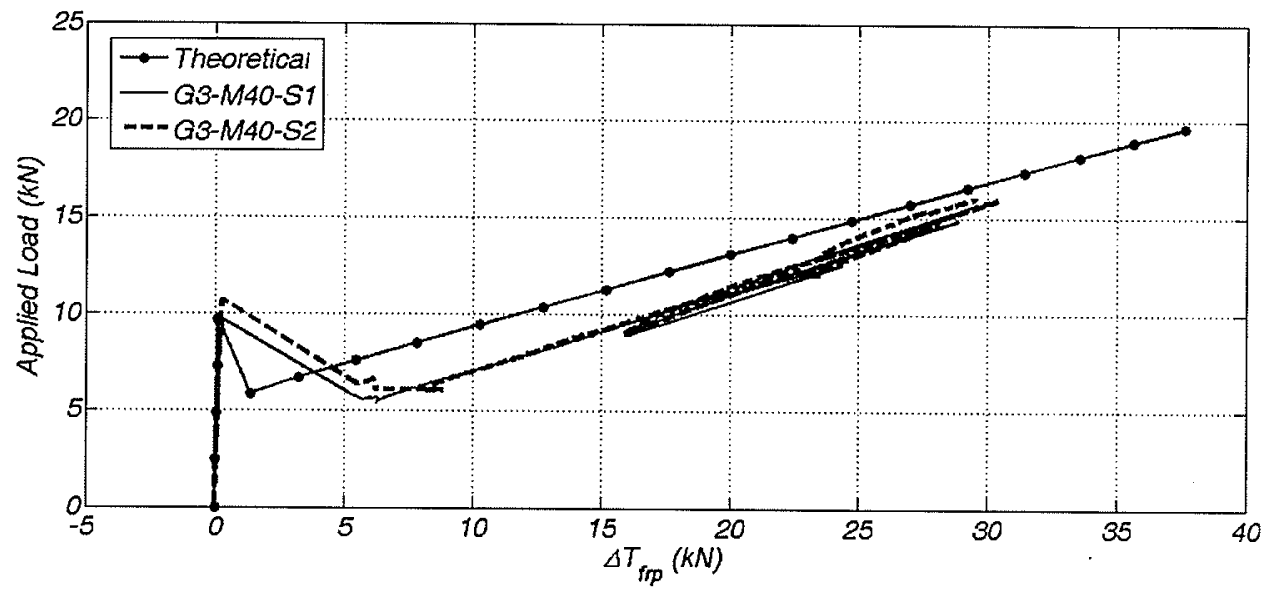

(c) Weathered Specimen Tested at $-40^{\circ} \mathrm{C}$, G3-M40

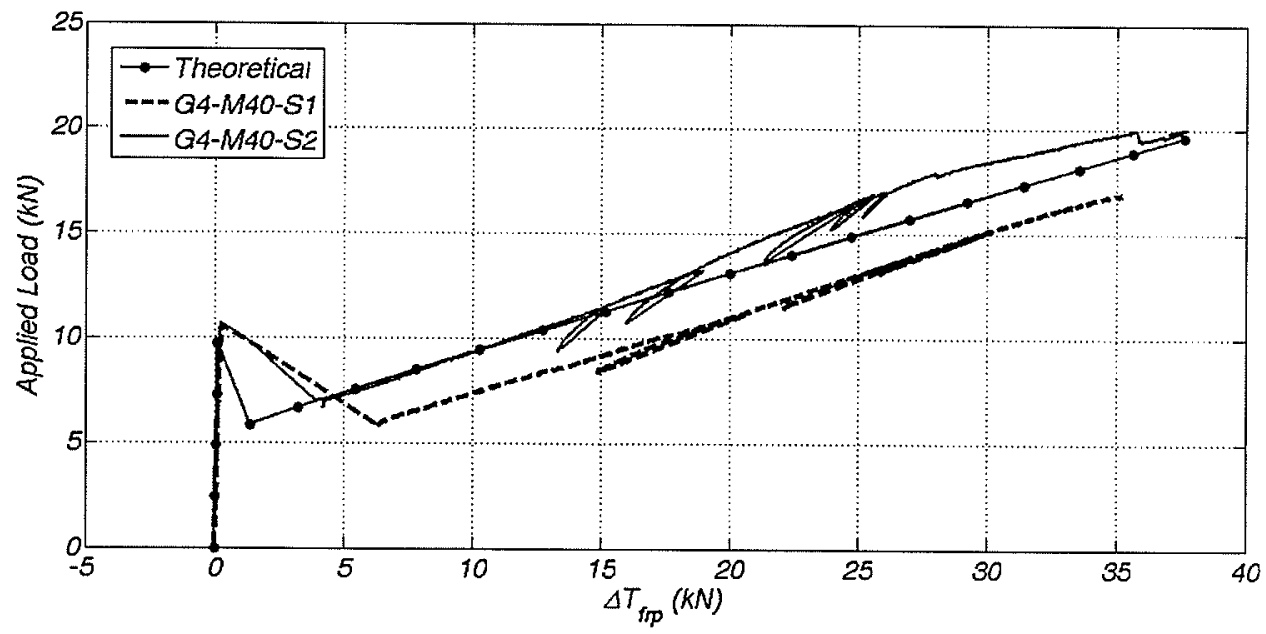

(d) Weathered Specimen Tested at $-40^{\circ} \mathrm{C}, \mathrm{G} 4-\mathrm{M} 40$

Figure 5.42 - Tendon Force Change with Applied Load 
It is clear from the results of Figure 5.42 that the progression of the tendon force gradient is consistent among all tests conducted on beams prestressed with GFRP tendons. This suggests that bond behaviour and bond strength have not altered as a result of the weathering process and testing temperatures adopted for these beams.

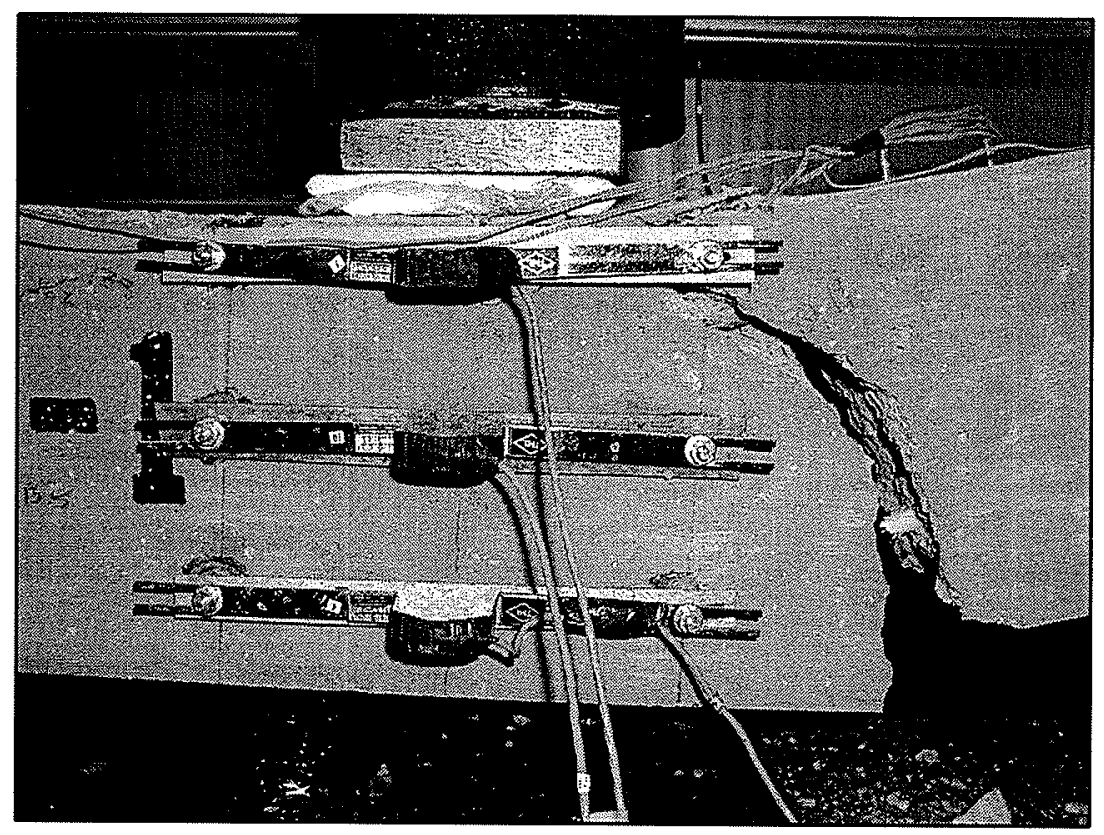

Figure 5.43 - Shear Failure (GC-AMB-S2)

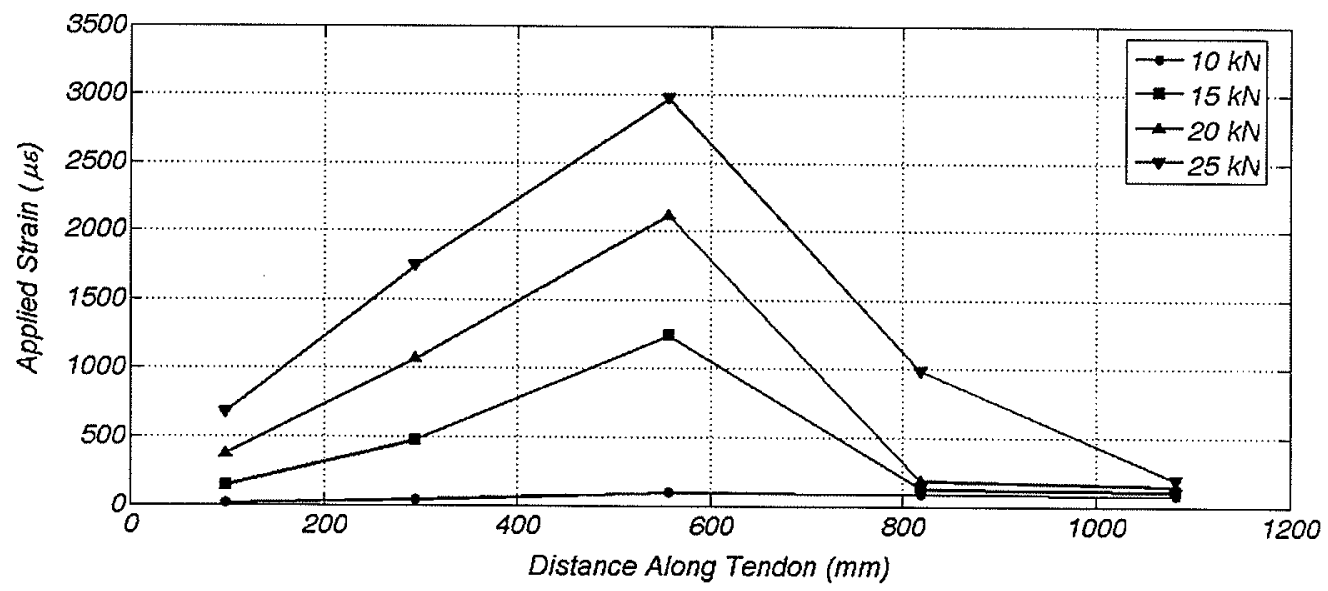

Figure 5.44 - Strain Profile Along CFRP Tendon (C1-P40-S1)

Conversely, strains within the transfer length of beams prestressed with CFRP tendons were found to increase during the flexural bond tests. Figure 5.44 describes this by illustrating strain 
gradients typically occurring along the length of a CFRP tendon. Slippage of the reinforcement was therefore observed for all CFRP prestressed beams tested in this research, as will be discussed in the following section.

\subsubsection{End Slip}

As anticipated and previously discussed from strain gradients recorded along the length of GFRP prestressed beams, slippage of the tendon was nonexistent during flexural bond tests. This was established on the basis of readings obtained from LVDTs monitoring slip at the tested end. It was further confirmed by monitoring the progression of a permanent marker reference point placed on the extruding portion of the tendon during the tests. As encircled in Figure 5.45, the point remained fixed with respect to the edge of the beam during all tests.

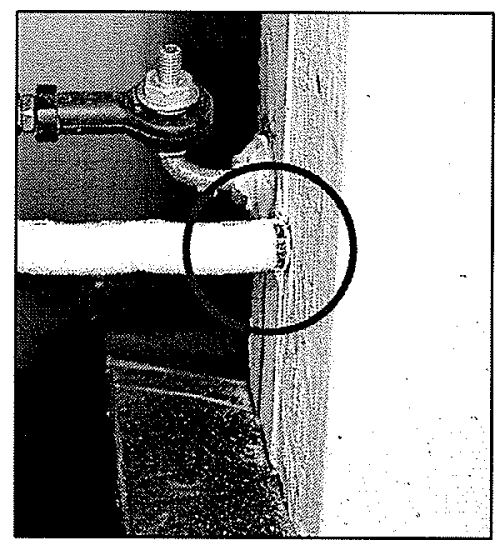

(a) Before Test

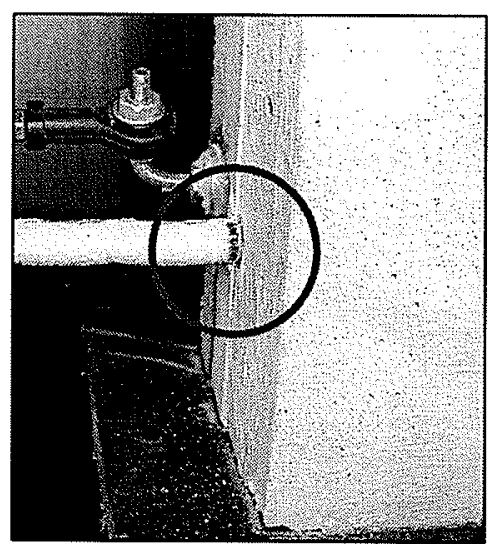

(b) $15 \mathrm{kN}$ Load Stage

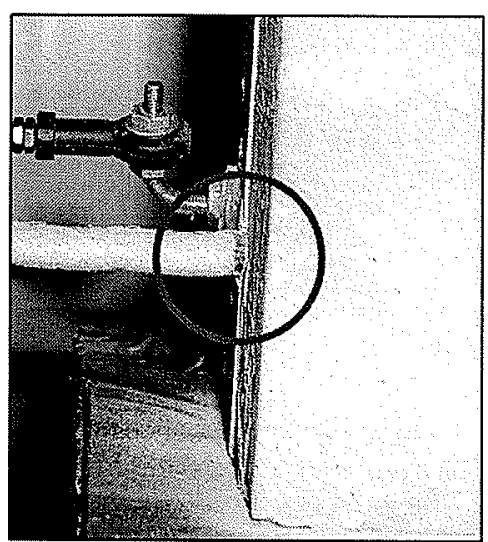

(c) After Test

Figure 5.45 - Progression of Slip (GFRP)

In contrast to that obtained from tests conducted on beams prestressed with GFRP tendons, considerable slip was observed at the tested end of those prestressed with CFRP tendons. The progression of slip as a function of the applied load for these beams is illustrated in Figure 5.46. The initiation of slip occurred slightly after cracking and continued gradually as the tests progressed. Although maximum loads differed among several of the tests conducted on CFRP prestressed beams, the loads at which slip initiated remained unaffected and consistent. It was 


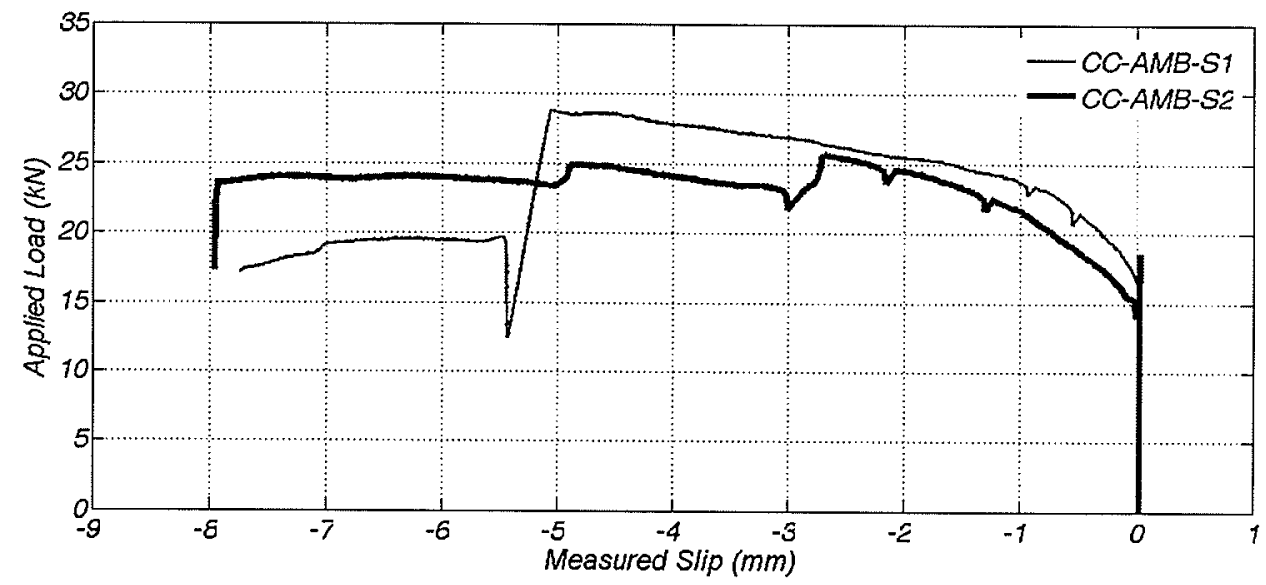

(a) Unweathered Specimens at Ambient Temperature

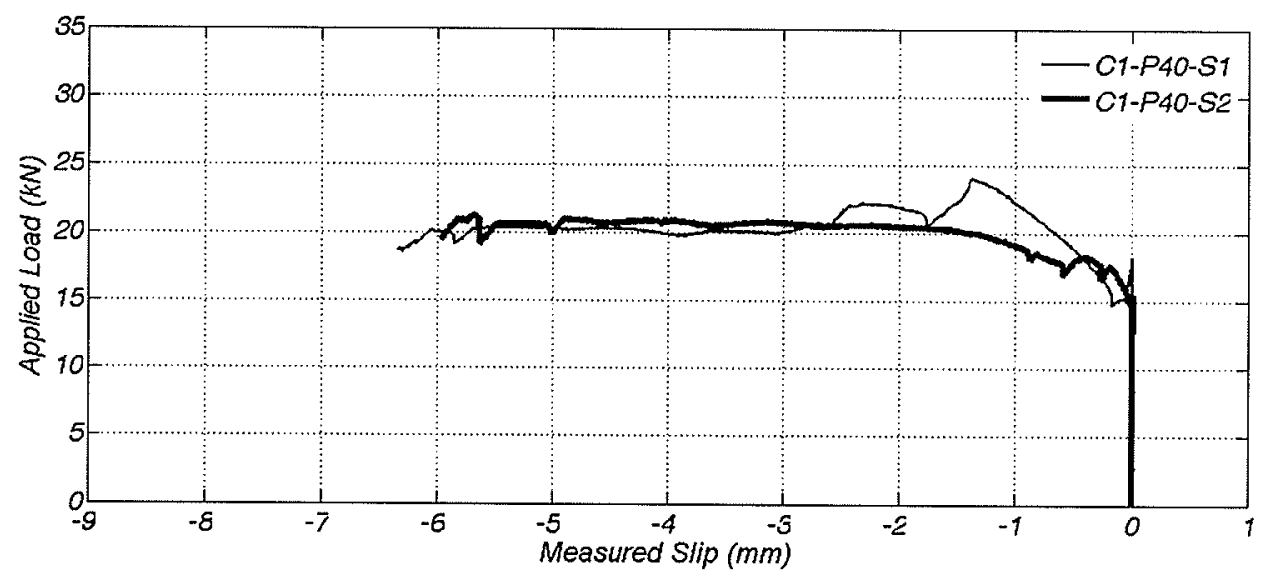

(b) Weathered Specimens at $+40^{\circ} \mathrm{C}$

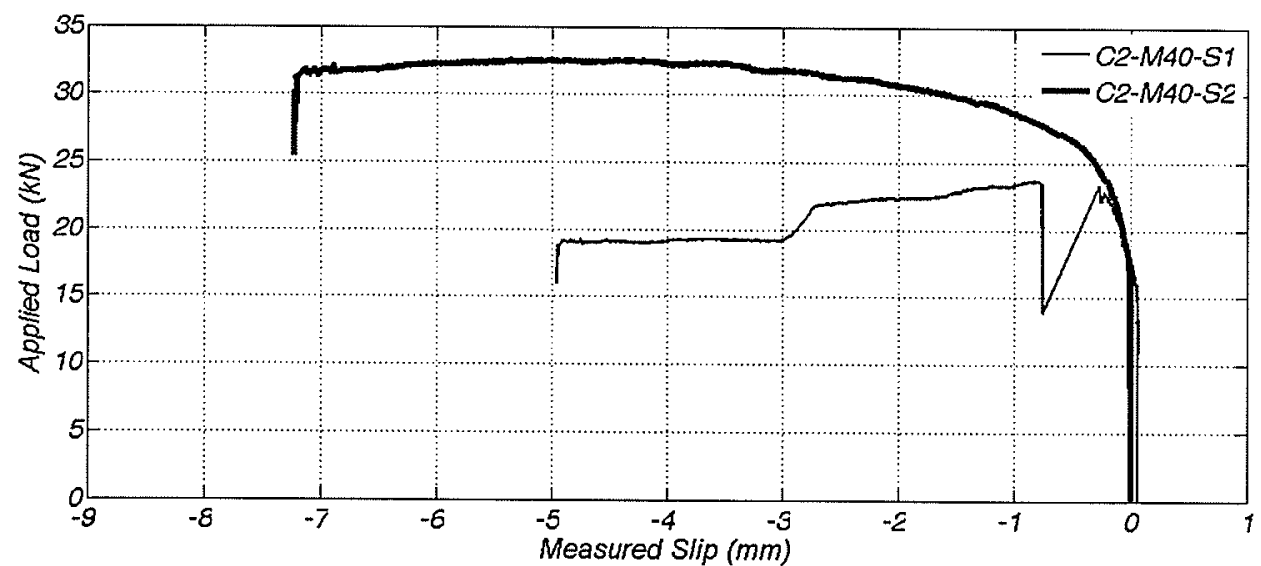

(c) Weathered Specimens at $-40^{\circ} \mathrm{C}$

Figure 5.46 - End Slip (CFRP) 
therefore concluded on the basis of these results that the weathering process and testing temperature level did not affect the bond strength of CFRP tendons to concrete.

The progression of a reference point on the tendon was also monitored during this set of tests. As illustrated in Figure 5.47, the point was found to move gradually towards the edge of the beam at various loading stages during the tests.

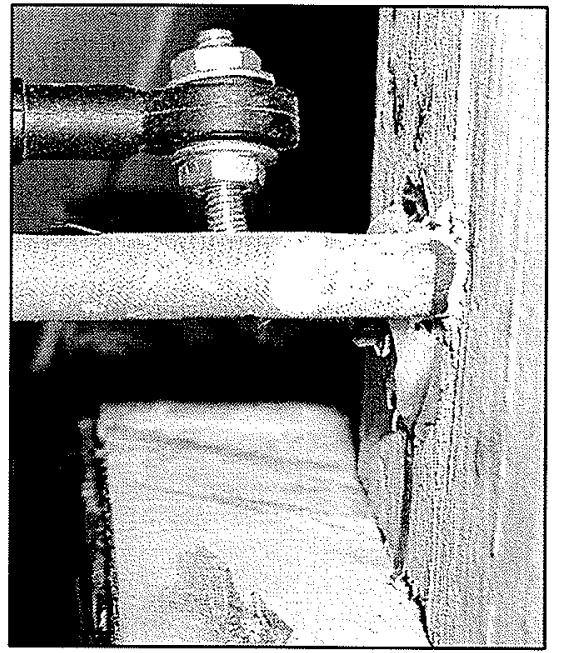

(a) Before Test

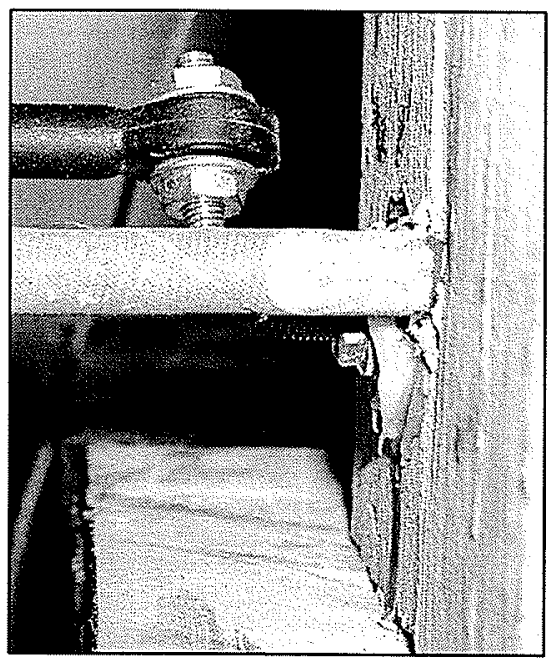

(c) $25 \mathrm{kN}$ Load Stage

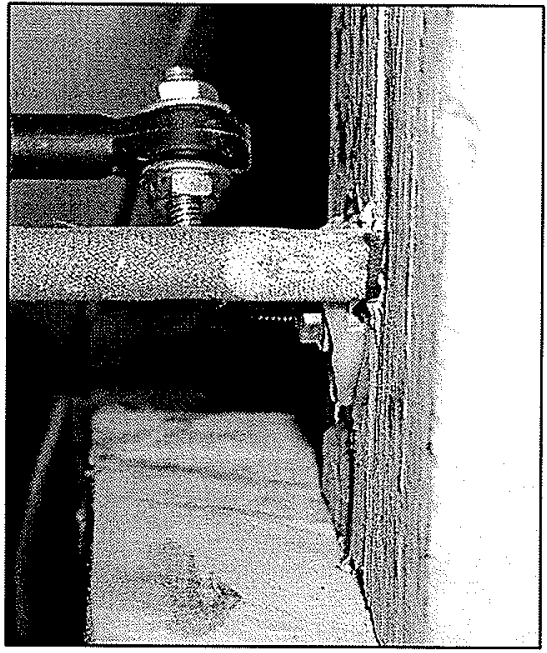

(b) $20 \mathrm{kN}$ Load Stage

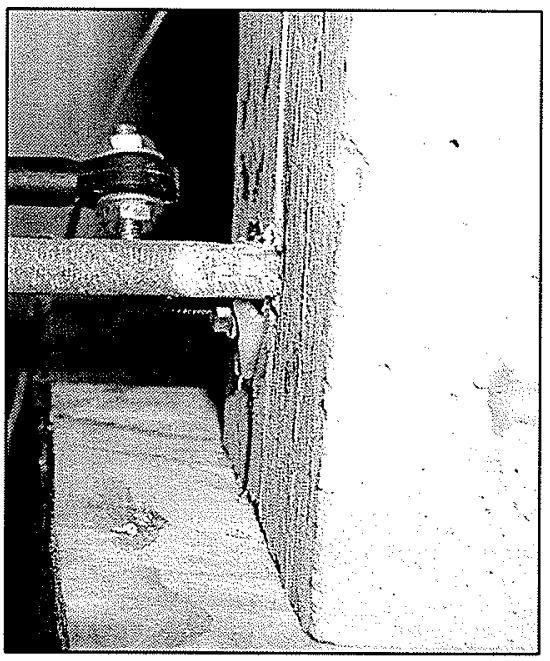

(d) After Test

Figure 5.47 - Progression of Slip (CFRP) 


\subsubsection{Crack Formation and Pattern}

The occurrence and development of cracks was closely monitored during all flexural bond tests. In all cases, the first crack appeared under the point of load where maximum moment occurs. The crack pattern observed for beams prestressed with GFRP tendons was distinct from that observed for those prestressed with CFRP tendons. The divergence transpired from the difference in material properties existing between individual tendons and discrete failure mechanisms of each beam.

\section{GFRP Prestressed Beams}

Numerous cracks formed within the tested span of beams prestressed with GFRP tendons. As the applied load was increased beyond the point of cracking, additional cracks gradually developed adjacent to the point of load where cracking initiated. These cracks were discovered between the point of load and the overhang support. When considering all flexural bond tests conducted on beams prestressed with GFRP tendons, the last crack was found to form once the applied load reached 80 to 90 percent of ultimate. This crack was the sole one to develop between the point of load and first support where slip was measured. The development of cracking is illustrated in Figure 5.48 with cracks numbered in the order they were typically observed to occur.

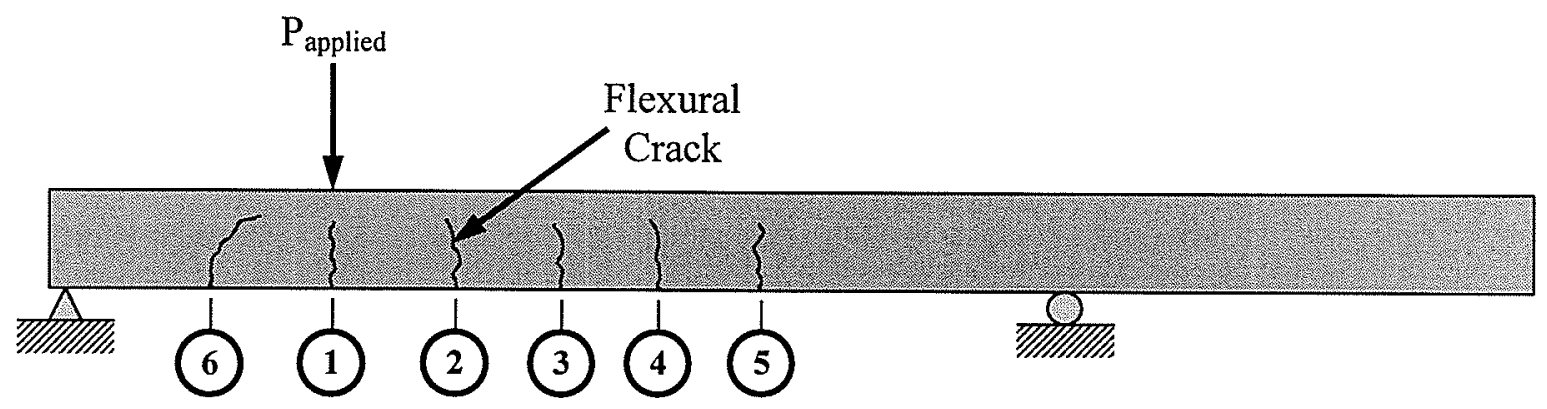

Figure 5.48 - Typical Crack Pattern and Progression - GFRP Prestressed Beams 
The limited amount of cracks that developed between the point of load and first support became the fundamental reason behind the absence of slippage for beams prestressed with GFRP tendons. Non-existence of cracks within this region of the beam during the majority of the test did not cause as many peaks in tendon stresses as that observed in the control beams cast at the University of Manitoba in the final stages of 2003. The development length was consequently superior to that predicted from the control beams and sufficient to allow rupture of the tendon before any signs of slippage. Nevertheless, consistency in strain gradients measured along the length of tendons among all tests was sufficient to conclude that bond strength was not affected by the weathering process and temperature level adopted during testing.

\section{CFRP Prestressed Beams}

Fewer cracks formed in beams prestressed with CFRP tendons than that observed in beams prestressed with GFRP tendons. In the majority of these tests, the crack under the point of load was the only one to develop as additional cracks were not observed when the load was increased beyond cracking. Rather, slippage of the tendon was found to increase as the load gradually progressed to a ceiling value. In some cases however, an additional crack formed between the point of load and first support, causing the ceiling load to be lower than that observed in beams developing a single crack under the loading point. Even so, the load initiating slippage of the reinforcement remained consistent throughout the series of tests conducted on beams prestressed with CFRP tendons. It therefore became apparent that the weathering process and testing temperature did not have a noticeable effect on the bond strength of CFRP tendons in a concrete environment.

The crack pattern for all beams tested in this research is illustrated in Figure 5.49. The figure draws attention to the fewer number of cracks recorded for beams prestressed with CFRP tendons. The loading position for both tests conducted on each beam is also shown in the figure 
by means of an arrow. This further points out the deficiency of cracks towards the first support for beams prestressed with GFRP tendons.

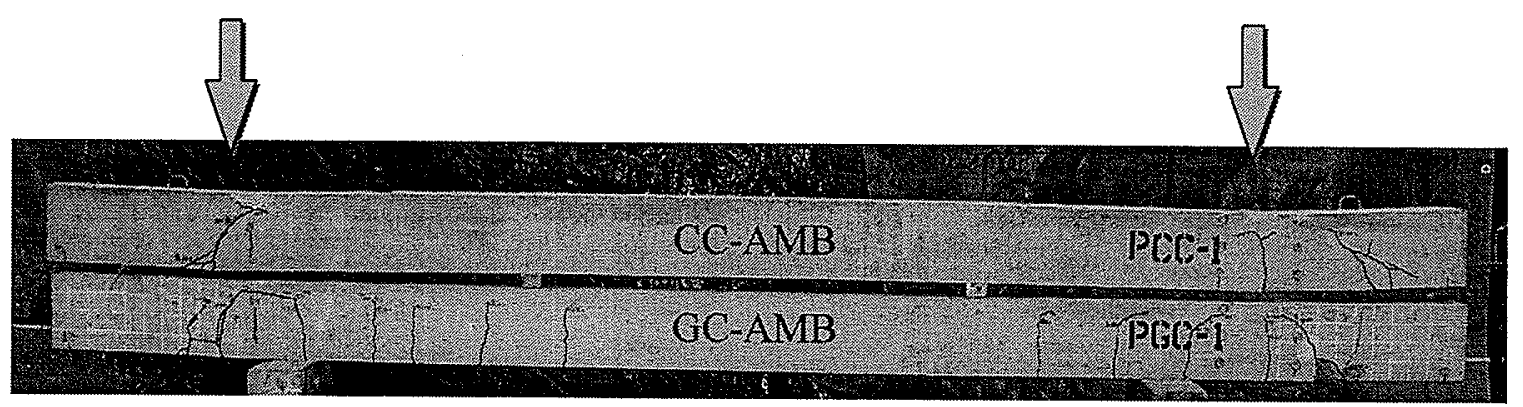

(a) Unweathered Specimens Tested at Ambient Temperature (CC-AMB \& GC-AMB)

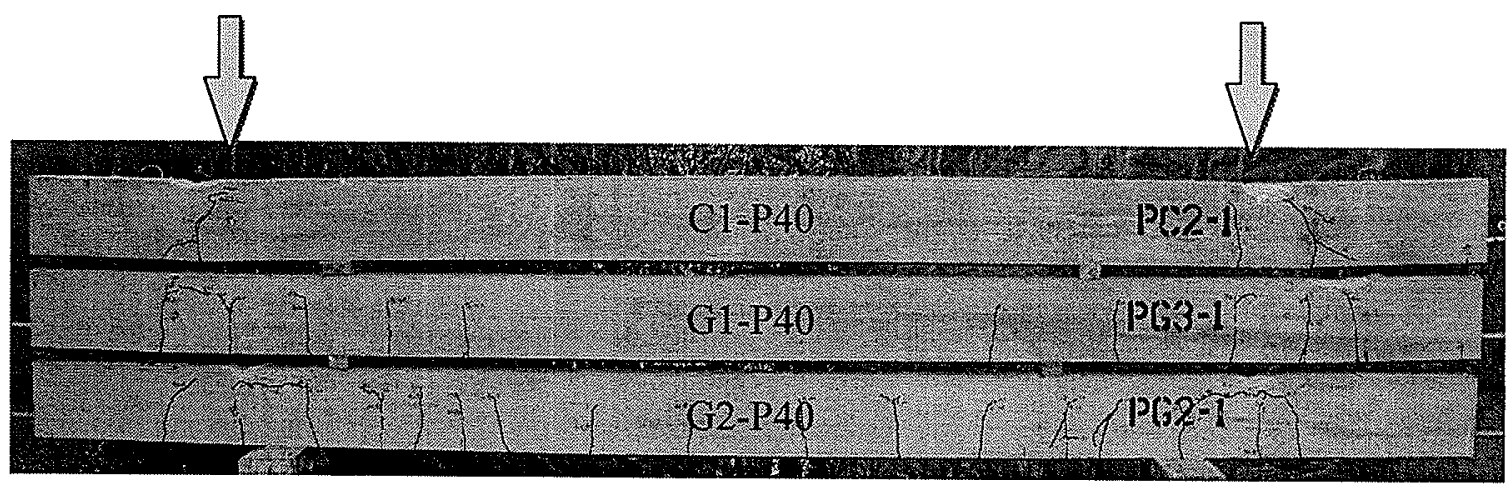

(b) Weathered Specimens Tested at $+40^{\circ} \mathrm{C}$ (C1-P40, G1-P40 \& G2-P40)

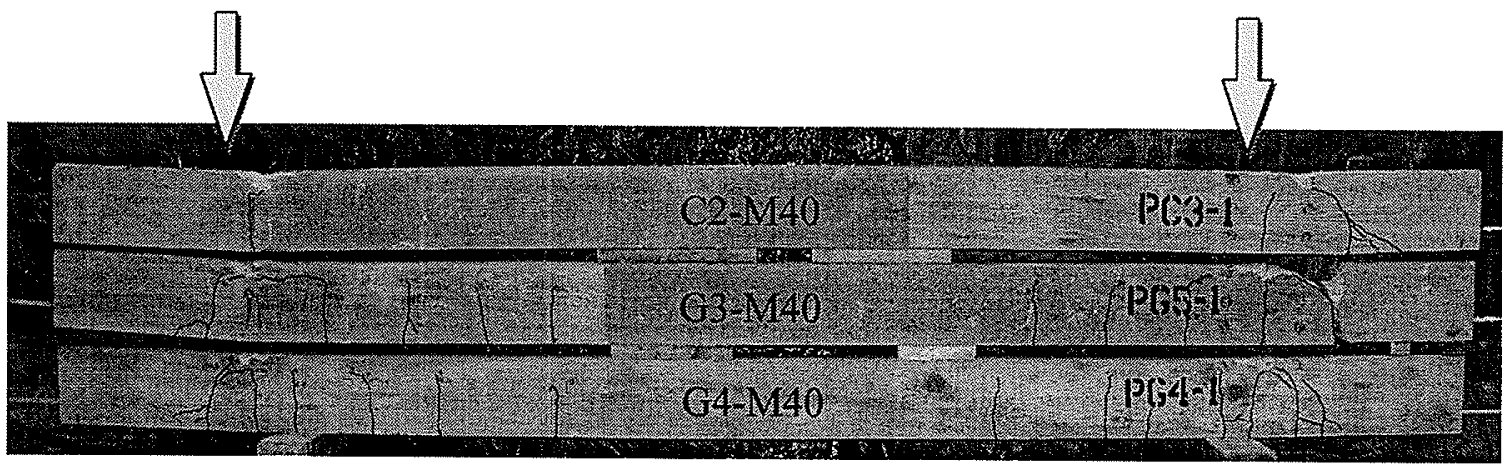

(c) Weathered Specimens Tested at $-40^{\circ} \mathrm{C}$ (C2-M40, G3-M40 \& G4-M40)

Figure 5.49 - Crack Pattern 


\section{Chapter 6}

\section{Conclusions and Recommendations}

\subsection{Summary and Conclusions}

Several conclusions were drawn from results presented and discussed in this thesis. More specifically, the current section will present conclusions pertaining to each phase of the research. The first phase includes the linear elastic and finite element analyses conducted to evaluate the amount of damage expected from thermal swelling of various FRP reinforcing bars in a concrete environment. The second phase covers an experimental program carried out to determine the potential effect of such damage on the bond strength of FRP reinforcing bars in concrete elements with minimum concrete cover provisions specified by the CSA S806 (2002) design code.

\subsubsection{Linear Elastic Analysis}

The linear elastic analysis presented in this thesis is based on expressions simplified by the assumption of linear elastic material behavior as well as axisymmetric and plane stress conditions. These expressions were used to study the progression of stress and damage within the concrete cover and determine thermal gradients causing crack initiation as well as splitting of the concrete cover. Critical temperature charts were developed from these gradients to determine the concrete cover required to prevent initiation of cracking and occurrence of the splitting phenomenon for temperature levels expected in practice and various material properties. A sensitivity analysis was also performed on the linear elastic model to determine material 
properties most susceptible to affect stress distribution and development of cracking within the cover. Considering the linear elastic analysis, conclusions listed below were drawn.

(1) Before cracks initiate within the cover, increasing the c/d ratio beyond 3.0 does not appreciably alter the concentration of stress at the surface of the reinforcement. Consequently, increasing the c/d ratio beyond this value was found to be inefficient in preventing cracks to initiate within the concrete cover. Results from the analysis clearly demonstrate that thermal gradients required to initiate cracking hardly increase with c/d ratios beyond 3.0 .

(2) Increasing the c/d ratio was found to be more efficient in preventing splitting of the concrete cover than preventing crack initiation. According to the analysis and limiting conditions for expressions defining it, thermal gradients required to cause splitting of the cover were found to increase consistently with concrete cover.

(3) According to the sensitivity analysis performed on expressions describing the linear elastic model material properties most susceptible to affect stresses and damage within the concrete cover include the concrete compressive strength as well as the transverse elastic modulus and transverse CTE of the reinforcement.

(4) It is clear from the sensitivity analysis that increasing the compressive strength or choosing an FRP reinforcing bar with smaller transverse elastic modulus or smaller transverse CTE is more efficient in preventing crack initiation within the concrete cover than increasing the $\mathrm{c} / \mathrm{d}$ ratio. 


\subsubsection{Finite Element Analysis}

The finite element analysis shares the same objective as the linear elastic analysis. Critical temperature charts similar to those obtained from the linear elastic model were also developed from the model and compared to those obtained from the linear elastic analysis. The comparison was performed to investigate inclusion of non-linear material behavior in the analysis, generalization of stress-strain relationships accounting for multiaxial states of stress and consideration of geometries most likely found in practice. The following conclusions were drawn on the basis of results presented for the finite element analysis.

(1) As for the linear elastic analysis, first principal stresses along the shortest concrete covers of the finite element model were found to be highest in the vicinity of the reinforcement before cracking. The stresses were also found to dissipate with distance from the reinforcement.

(2) Stress distributions obtained from each analysis differ in some respects. The finite element analysis was found to distribute stresses more evenly within the cover. Stresses appear smaller at the interface and dissipate at a lower rate than that described by linear elastic expressions. This produces slightly larger thermal gradient predictions at the onset of cracking.

(3) Geometry of the finite element model varies around the reinforcement with shortest covers defining the side and bottom of the model. Accordingly, stress distributions along the largest concrete cover were compared to those along the shortest concrete covers. Results indicate that stresses are significantly close to those obtained along the shortest concrete covers. 
(4) As the stress distribution remained invariant between the largest and shortest covers of the model, it was concluded that increasing the $\mathrm{c} / \mathrm{d}$ ratio is inefficient in preventing crack initiation within the concrete cover. The result is similar to that obtained from the linear elastic analysis with thermal gradients at the onset of cracking hardly increasing with concrete cover.

(5) The finite element analysis also revealed that increasing the c/d ratio was more efficient in preventing the splitting phenomenon than preventing crack initiation within the cover. Critical temperature charts obtained from the analysis show that thermal gradients at splitting consistently increase with concrete cover.

(6) For larger c/d ratios, critical temperature charts show that thermal gradients from the finite element analysis are smaller than those obtained from the linear elastic analysis at splitting. The divergence is attributed to consideration of a biaxial state of stress in the finite element model. Presence of compressive stresses within the cover can reduce the tensile strength of concrete, accelerate development of cracking within the cover and cause smaller thermal gradients at splitting.

\subsubsection{Experimental Program}

The experimental program was aimed at determining the bond strength remaining after subjecting prestressed beams with minimum concrete cover provisions to thermal cycles typically expected in the Canadian climate. All beams in the project were designed according to the CSA S806 (2002) design code. Two control beams were cast during the month of December 2003 to determine development length of the GFRP and CFRP reinforcement used in the research. An additional set of 10 beams was cast during the month of April 2004 and subjected to thermal cycles ranging between $-40^{\circ} \mathrm{C}$ and $40^{\circ} \mathrm{C}$. These additional beams were regularly inspected for damage until thermal cycling was complete. Inspections were performed with a $0.1 \mathrm{~mm}$ accurate 
hand held microscope. A non-destructive wave propagation method called Impact Echo was also used to identify possible damage in the transfer region of the beams. Results from the method are still being analyzed and will be included in future research at the University of Manitoba.

(1) No damage was detected when inspecting beams intended for the weathering process. Although cracking was expected to commence upon release of prestress. The absence of damage is mainly attributed to the relatively low transverse elastic modulus of the reinforcement. The modulus typically ranges between 2,000 and $4,000 \mathrm{MPa}$ but extensive work is still required to establish a widely accepted standard method for accurately determining this material property for FRP reinforcement.

(2) During the weathering process, strain gauges were placed on the reinforcement to measure and confirm transverse CTE values. While GFRP reinforcing bars had a transverse CTE close to that listed by the manufacturer $\left(29.5 \mu \varepsilon /{ }^{\circ} \mathrm{C}\right)$, CFRP reinforcing bars demonstrated a transverse CTE $\left(29.2 \mu \varepsilon /{ }^{\circ} \mathrm{C}\right)$ much lower than that listed by the manufacturer $\left(90 \mu \varepsilon /{ }^{\circ} \mathrm{C}\right)$. This enforces the reason for which damage in beams prestressed with CFRP reinforcement was less than that expected from analyses presented in this thesis.

(3) Two of the beams intended for the weathering process were set aside and not subjected to thermal cycles. These beams served as reference specimens to evaluate the effect of thermal cycling on bond strength of FRP reinforcement in concrete. Deviation of results between reference and weathered specimens were not significant enough to suggest a noticeable trend in bond strength due to thermal cycling and related damage of the concrete cover.

(4) As expected from the design and location of load, beams reinforced with CFRP failed by slippage of the reinforcement. However, beams reinforced with GFRP were found to fail 
by rupture of the reinforcement. For these beams, potential degradation in bond strength was measured on the basis of strain gauge readings obtained on the surface of the reinforcement between the support and loading point.

(5) Beams in this research were subjected to thermal gradients typically expected in the Canadian climate and designed with minimum cover requirements as well as maximum permissible prestressing levels for each reinforcing bar. Under these conditions, results from the experimental program demonstrate that the CFRP and GFRP reinforcing bars tested in this program have excellent bond performance in a concrete environment without deterioration arising from differential swelling.

\subsection{Recommendations for Future Research}

The current thesis established thermal compatibility of FRP reinforcement on the basis of linear elastic and finite element analyses. Through an experimental program it also demonstrated that CFRP and GFRP reinforcement have excellent bond performance in a concrete environment. However, the research should be considered as a foundation for further research in the field of thermal compatibility with several recommendations prevailing.

(1) The finite element analysis should be elaborated to consider a wider range of material properties. Due to time constraints, results of the analysis were limited to transverse CTE values of 55 and $90 \mu \varepsilon /{ }^{\circ} \mathrm{C}$ and a transverse elastic modulus of $3,000 \mathrm{MPa}$ for the FRP reinforcement as well as concrete compressive strengths ranging from 30 to 50 MPa.

(2) According to analyses presented in this thesis, thermally induced stresses and related damage are particularly sensitive to the transverse elastic modulus of FRP reinforcement. 
A standard method for determining this property should therefore be developed to determine this material property.

(3) Once the glass transition temperature is reached, polymeric resin embedding fibers within FRP reinforcement will start to loose its mechanical properties. Due to the limited number of experiments reported in the literature on this topic, additional research is required to determine whether properties of the polymeric resin can be affect for thermal cycles and reinforcing bars considered in this project. Softening of the resin under thermal increase can reduce the transverse elastic modulus of FRP reinforcement and moderate tensile splitting stresses within the concrete cover. The extent of damage predicted from analyses considered in this research would lessen and clarify the absence of damage observed on the external surface of flexural specimens in this research.

(4) The current research has ignored the influence of bar spacing within the concrete cover. It is therefore recommended for future research to consider thermal swelling of a second bar within the concrete cover and its associated damage. Recommendations on bar spacing for various material properties can then be achieved. 


\section{References}

1) ADINA R\&D, Inc., ADINA User Interface, ADINA R\&D, Inc., Watertown, MA, USA, 2001, 398 pp.

2) Agarwal, B.D. and Broutman, L.J., "Analysis and Performance of Fibre Composites." Second Edition, John Wiley \& Sons, New York, NY, USA, 1990, 472 pp.

3) ASTM C39/C39M-04a, "Standard Test Method for Compressive Strength of Cylindrical Concrete Specimens." Annual Book of ASTM Standards, Vol.04.02, Easton, MD, USA, 2004, pp. 18-22.

4) ASTM C469-02, "Standard Test Method for Static Modulus of Elasticity and Poisson's Ratio of Concrete in Compression." Annual Book of ASTM Standards, Vol.04.02, Easton, MD, USA, 2002, pp. 242-250.

5) ASTM C496/496M-04, "Standard Test Method for Splitting Tensile Strength of Cylindrical Concrete Specimens." Annual Book of ASTM Standards, Vol.04.02, Easton, MD, USA, 2004, pp. 268-271.

6) ASTM E1512-01, "Testing Bond Performance of Adhesive-Bonded Anchors." Annual Book of ASTM Standards, Vol.04.11, Easton, MD, USA, 2001, pp. 664-668.

7) Aiello, M.A., "Concrete Cover Failure in FRP Reinforced Beams Under Thermal Loading." Journal of Composites for Construction, Vol.3, No.1, 1999, pp. 46-52.

8) Canadian Standards Association, A23.3-94 - Design of Concrete Structures, Canadian Standards Association, Toronto, ON, Canada, 1994, 220 pp.

9) Canadian Standards Association, S806-02 Design and Construction of Building Components with Fibre-Reinforced Polymers, Canadian Standards Association, Toronto, ON, Canada, 2002, 177 pp.

10) Collins, M.P., Mitchell, D., Prestressed Concrete Structures, Response Publications, ON, Canada, 1997, 766 pp. 
11) Cook, R.D., Malkus, D.S., Plesha, M.E., Witt, R.J., Concepts and Applications of Finite Element Analysis, $4^{\text {th }}$ ed., John Wiley \& Sons, New York, NY, USA, 1995, 627 pp.

12) Huebner, K.H., Thornton, E.A., Byron, T.G., The Finite Element Method for Engineers, Third Edition John Wiley \& Sons, New York, NY, USA, 2001, 719 pp.

13) Gentry. R.T., Husain, M., "Thermal Compatibility of Concrete and Composite Reinforcement." Journal of Composites for Construction, Vol.3, No.2, 1999, pp. 82-86.

14) Katz, A., "Bond Mechanism of FRP Rebars to Concrete." Journal of Materials and Structures, 1999, Vol.32, pp. 761-768.

15) Katz, A., Berman, N., "Modelling the Effect of High Temperature on the Bond Strength of FRP Reinforcing Bars to Concrete." Journal of Cement and Concrete Composites, 2000, Vol.22, pp. $433-443$.

16) Kupfer, H., Hilsdorf, H.K. and Rüsch, H., "Behaviour of Concrete under Biaxial Stress." Proceedings, ACl Journal, Vol. 66, No. 8, August 1969, pp.656-666.

17) Logan, D., "Acceptance Criteria for Bond Quality of Strand for Pretensioned Prestressed Concrete Applications." PCl Journal, v42, n2, 1997, pp. 52-90.

18) MacGregor, J.G., Bartlett, F.M., Reinforced Concrete Mechanics and Design, First Canadian Edition., Prentice Hall, Scarborough, ON, Canada, 2000, 1042 pp.

19) Mao, M., Taylor, C.A., "Non-Linear Seismic Cracking Analysis of Medium-Height Concrete Gravity Dams." Journal of Computers and Structures, Vol.64, No.5/6, 1997, pp. 1197-1204.

20) Nanni, A., Focacci, F., Aiello, M.A., "Effects of Thermal Loads on Concrete Cover of FibreReinforced Polymer Reinforced Elements: Theoretical and Experimental Analysis." $\mathrm{ACl}$ Materials Journal, Vol. 98 , No. 4, 2001, pp. 332-339.

21) Popovics, S., "A Review of Stress-Strain Relationships for Concrete." ACl Journal, Vol. 67, No. 3,1970 , pp. $243-248$.

22) Popovics, S., Strength and Related Properties of Concrete: A Quantitative Approach, John Wiley \& Sons, New York, NY, USA, 1998, 535 pp. 
23) Rahman, A.H., Kingsley, C.Y., and Taylor, D.A., "Thermal Stress in FRP-Reinforced Concrete." Proceedings, Canadian Society for Civil Engineering. Annual Conference, Canadian Society for Civil Engineering, Montreal, 2,1995, pp. 605-614.

24) de Schutter, G., Matthys, S., Taerwe, L., "Two-Dimensional Analysis of Thermal Incompatibility Between FRP Reinforcement and Concrete." Proceedings of the Second International DIANA Conference on Finite Elements in Engineering and Science, Amsterdam, June 1997, pp. 4-6.

25) Shah, S.P., Swartz, S.E., Ouyang, C., Fracture Mechanics of Concrete, John Wiley \& Sons, New York, NY, USA, 1995, 552 pp.

26) Thorenfeldt, E., Tomaszewicz, A., Jensen, J.J., "Mechanical Properties of High Strength Concrete and Application in Design." Proceedings of the Symposium "Utilization of High Strength Concrete" Stavanger, Norway, June 1987, pp.149-159.

27) Timoshenko, S.P., Goodier, J.N., Theory of Elasticity, $3^{\text {rd }}$ ed., McGraw-Hill, New York, NY, USA, 1970, $567 \mathrm{pp}$.

28) Zienkiewicz, O.C., Taylor, R.L., The Finite Element Method, $5^{\text {th }}$ ed., Butterworth-Heinemann, Oxford, MA, USA, 2001, $459 \mathrm{pp}$. 